\title{
REHABILITATION IN ADVANCED DEMENTIA THROUGH COMPUTER-ASSISTED EXERGAMING WITH ABLE-X: \\ A COLLECTIVE CASE STUDY
}

\author{
By \\ JEANETTE DRURY-RUDDLESDEN
}

A thesis submitted to the Victoria University of Wellington in fulfilment of the requirements for the degree of Doctor of Philosophy in Health

Victoria University of Wellington 2017 


\begin{abstract}
Psychosocial interventions have been found to have similar outcomes as pharmacological interventions for people with dementia (PWD). Cognitive stimulation has been found to benefit cognition and there is evidence of self-reported quality of life (QOL) and wellbeing improvement in this population. However, little research has been carried out in exploring the association between QOL and computer-assisted exergaming in PWD. This study explored if a programme of computer-assisted exergaming interventions, utilising exergaming technology (Able-X), as an adjunct to existing activities and treatments, could deliver improvements in QOL, including cognitive and physical function for 10 people diagnosed with dementia.
\end{abstract}

An ontological approach of social pragmatism, combined with interpretive epistemology, within a collective convergent parallel case study (CS) methodological design was used. The study was carried out in three aged care residential units in New Zealand (NZ) that catered for people with dementia. As far as possible the principle of errorless learning (EL) (Terrace, 1963) was applied to the gaming environment when utilising the Able-X gaming interface, which was not entirely errorless. EL is based on the principle that it is difficult for people with impaired memory to remember and correct errors made while trying to learn or relearn something.

Pre-and post-gaming qualitative data were collected through semi-structured interviews with lead care staff and the next of kin for each participant, health record review, observation of video footage of the gaming experiences and computer-generated exergaming scores. In addition, several quantitative measures were used: Mini-Mental State Examination-2-BV, Global Deterioration Scale, Functional Assessment Staging Test, Quality of Life-AD, Cornell Scale for Depression in Dementia and the Rating Scale for Anxiety in Dementia. Data were generated over a 10-month period (excluding postscript comments). Data triangulation included application of the theoretical framework of 'embodied selfhood' (Kontos, 2003, 2004) to demonstrate participants' agency through intentionality of individualistic bodily movements, gestures and a sentient affinity with the social environment, and through habitus of the social relational body that implicitly understands the socio-cultural ways of being-in-the-world. 
The analysis revealed five emergent themes and six novel concepts that lend themselves to further inquiry. The first three themes: 'Sentient interaction with the social gaming environment', 'Initiation and gaming advancement in partnership', and 'Levels of gaming intentionality', focused on the gaming environment, including gaming initiation, engagement and progress. The other two themes: 'Hand-eye coordination' and 'Aspects of QOL', evidenced the similarities and differences across each case in terms of the main findings.

The key findings were significant improvements in hand-eye coordination, global QOL, and anxiety levels. Furthermore, cognitive scores improved for five participants and depression scores for seven participants, however, these improvements were not significant. In addition, there was qualitative evidence of enhanced motivation to reengage in psychosocial social activities for all participants, improved behavioural symptoms of dementia for nine participants, enhanced communication for nine participants and enhanced functioning of skills relating to activities of daily living tasks for seven participants.

This study demonstrated that improved hand-eye coordination and overall QOL were the results of computer-assisted exergaming with Able-X and EL strategies. Irrespective of the stage of dementia, significant benefits can be achieved through either solo or small group facilitated exergaming sessions.

Key Words: Able-X (ableX), case study, computer, dementia, embodied selfhood, errorless learning, exergaming, hand-eye coordination, quality-of-life, rehabilitation. 


\section{ACKNOWLEDGEMENTS}

First and foremost, I would like to acknowledge my gratitude to the primary participants involved in this study. The moments we shared are captured and their embodied selves will live on in the pages of this thesis and in the poem, I wrote, based on my experience of doing this research (p. 231). My appreciation is also extended to the family members and registered nurse informants.

I would like to thank my supervisors for their guidance, encouragement, and support throughout the process of conducting the study and writing up of this thesis. To my primary supervisor, Dr Kay de Vries, you shared not only your wealth of knowledge and expertise, but also your unfailing support, constant encouragement, accessibility, sense of humour and belief in me when I lost confidence in myself. To my secondary supervisor, Dr Kathy Nelson, thank you for your encouragement, guidance, accessibility, kindness, and positive attitude. I would also like to acknowledge the support and encouragement given to me from friends and colleagues. Your words of encouragement kept me motivated throughout this journey. Particular thanks go to Michelle Kim and Helen Rook, I was blessed to have your company on this journey and no matter where we end up in the world, and I know we will always be friends. Thanks also extend to my good friends Sheila Hunt, for proof reading the first draft of my literature review, to Amy Jackson, for proof reading second draft chapters 1, 3, and 4, and to Dr Jan Duke for proof reading the full third draft of this thesis and for being such a motivating influence.

In addition, I would like to acknowledge Callaghan Innovation Fund, NZ, for awarding me The Callaghan Innovation Scholarship 2014, established under the Callaghan Innovation Act 2012, and for part funding this study. Acknowledgements are also extended to Marcus King from Medical Device Technology, Callaghan Innovation, and my industry mentors from Im-Able Ltd., Sunil Vather, and Elliott Kernohan.

Finally, to my wonderful family, both here in NZ and in the UK, a very big Yorkshire "ta very much", for your unconditional love, support and encouragement and to the newest member of the family, my grandson Charlie, 19 months old already, I am so glad you like books because this one is a big one! 
TABLE OF CONTENTS

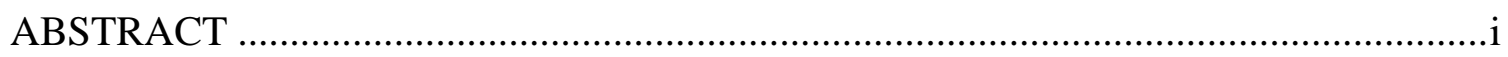

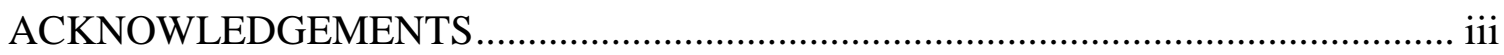

TABLE OF CONTENTS ………………………………….................................

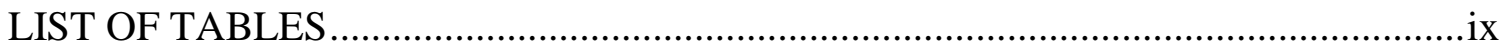

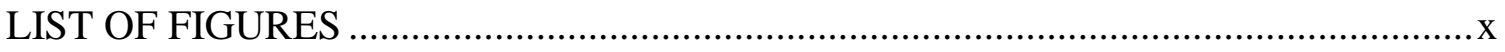

GLOSSARY OF ABBREVIATIONS ………………………………………….......

GLOSSARY OF TERMS ...................................................................................

CHAPTER ONE: INTRODUCTION AND BACKGROUND LITERATURE ..................1

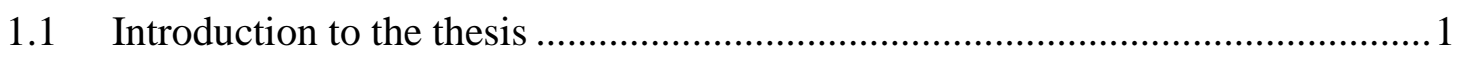

1.2 Research interest from a professional perspective .........................................2

1.3 Dementia demographics and prevalence .........................................................

1.4 Defining and diagnosing dementia .............................................................6

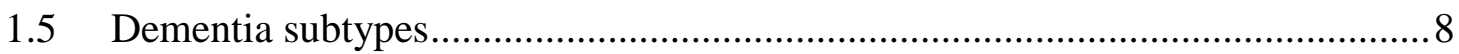

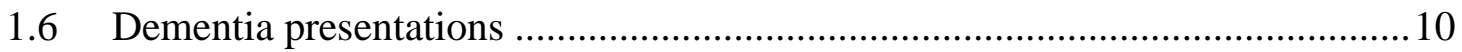

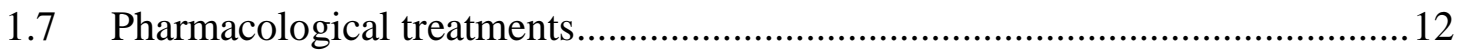

1.8 Global disease care burden and economic impact............................................. 14

1.9 Risk factors: non-modifiable and modifiable ………………………………..... 16

1.10 Philosophical positioning of thought, thinking, mind and body ........................19

1.11 Dementia and stigma (external and internal) ...................................................2

1.12 Memory and learning ............................................................................22

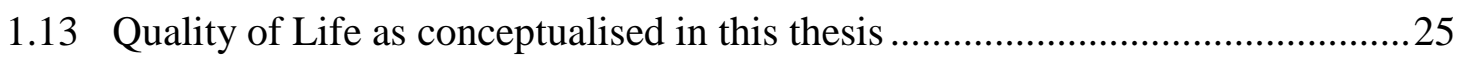

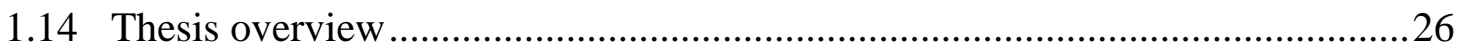

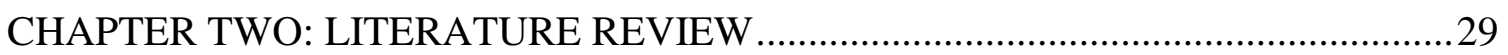

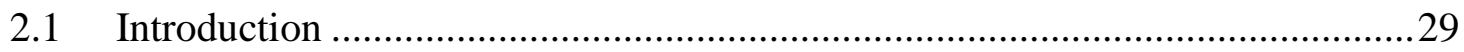

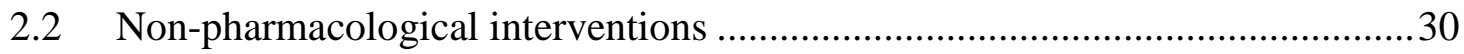


2.2.1 Cognitive stimulation: The evidence base ................................................ 31

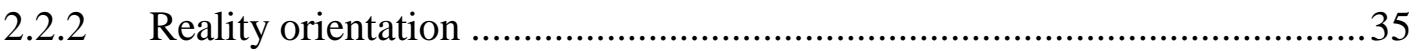

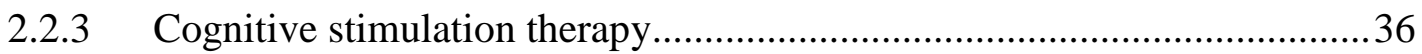

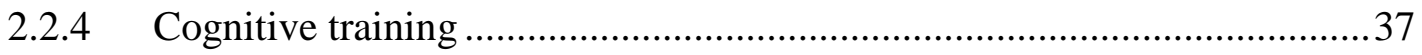

2.2.5 Computer brain training and gaming environment ..................................38

2.2.6 Cognitive rehabilitation through computer-assisted exergaming ................39

2.2.7 Cognitive rehabilitation through errorless learning ................................ 45

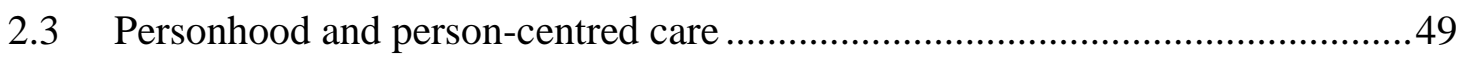

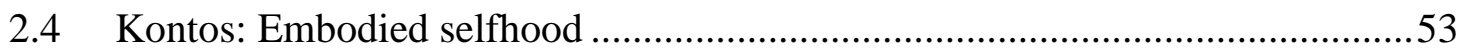

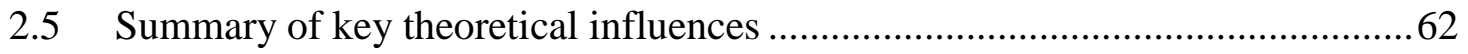

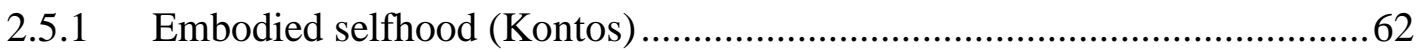

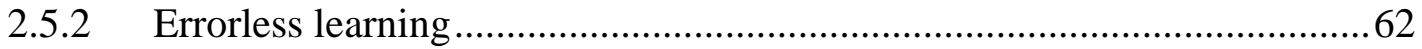

2.5.3 Multi-sensory enriched environment for cognitive rehabilitation ..............62

2.5.4 Ethical and moral influences of Kitwood ................................................63

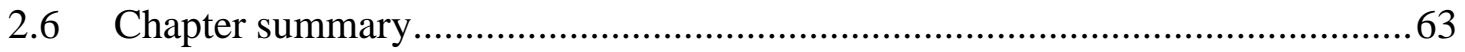

CHAPTER THREE: METHODOLOGY AND METHODS ….......................................65

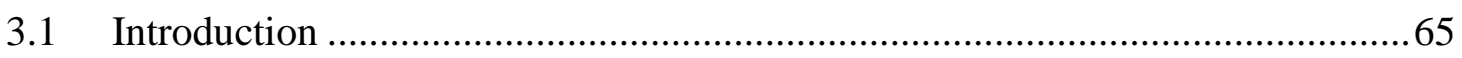

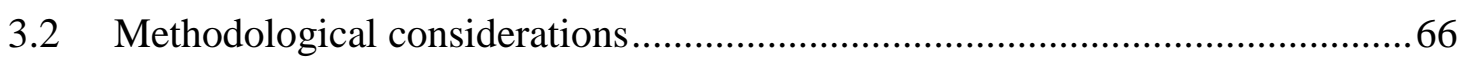

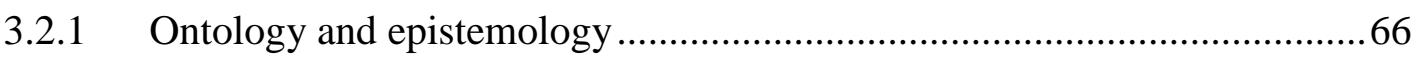

3.2.2 Methodology: Making a case for Case Study .......................................... 70

3.2.3 Case study question, purpose and components ...................................... 72

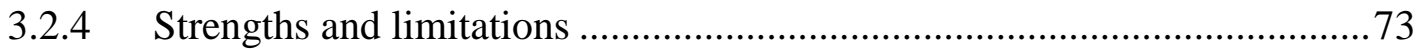

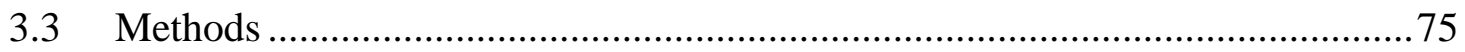

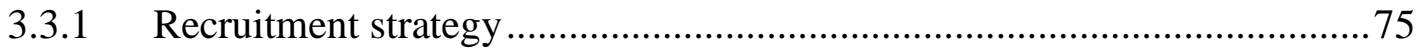

3.3.2 Exergaming with Able-X: The intervention .......................................... 79

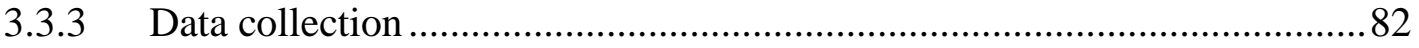

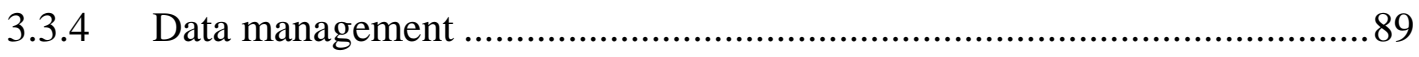




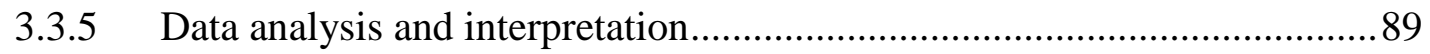

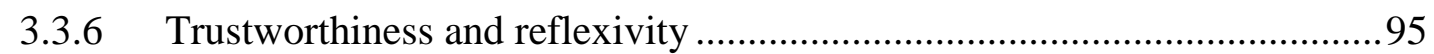

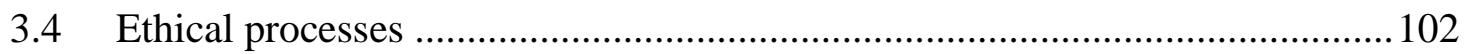

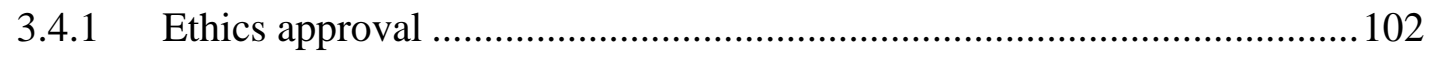

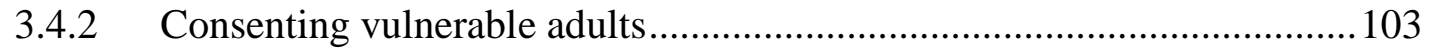

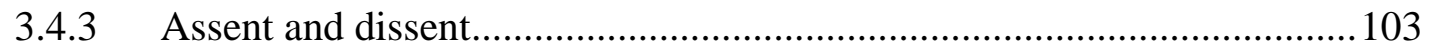

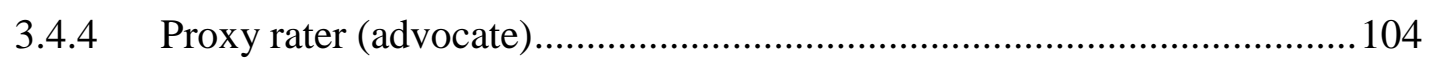

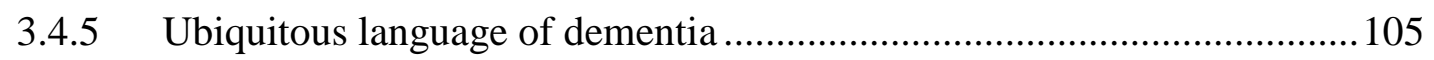

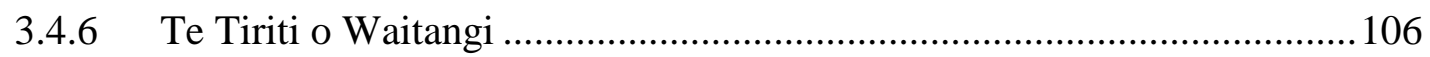

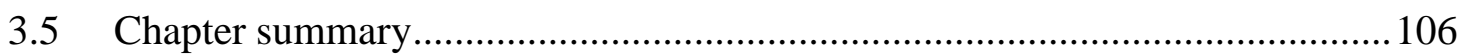

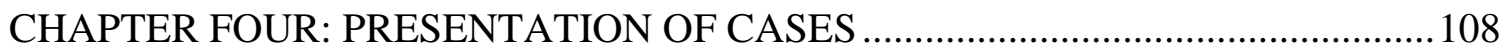

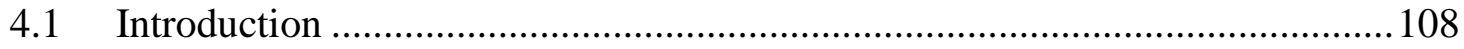

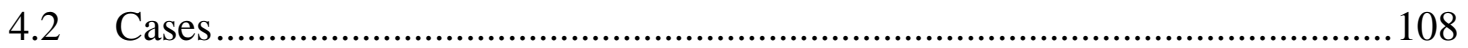

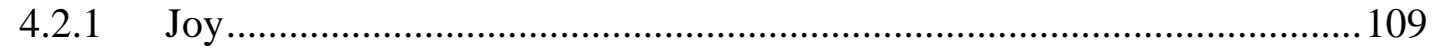

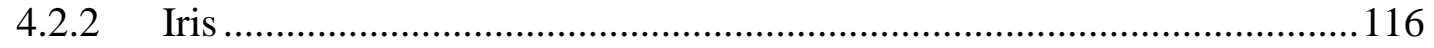

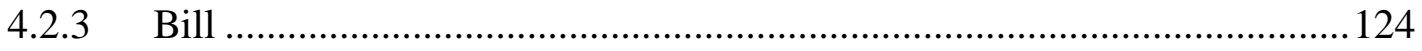

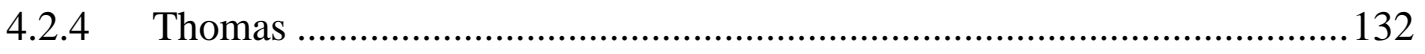

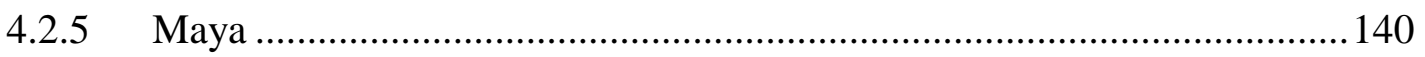

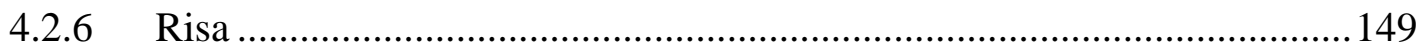

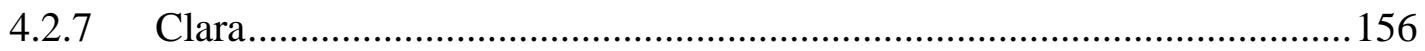

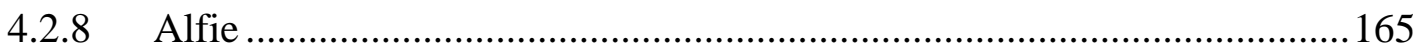

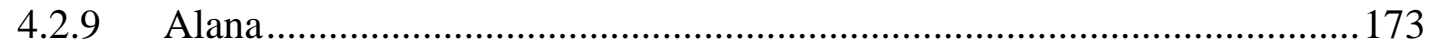

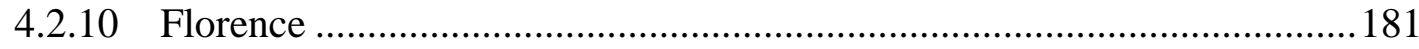

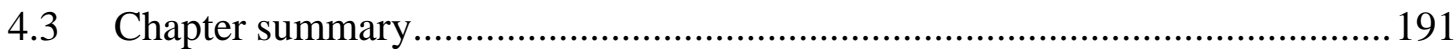

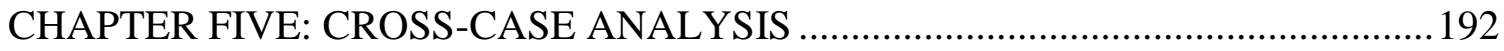

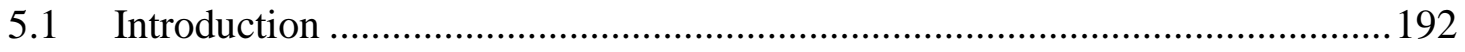

5.2 Demographic and personal characteristics ............................................... 193 
5.3 Clinical characteristics.

5.4 Engagement with computer-assisted exergaming with Able-X ....................... 198

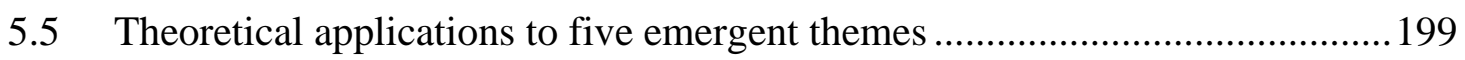

5.5.1 Theme 1. Sentient interaction with the social gaming environment ........199

5.5.2 Theme 2. Initiation and gaming advancement in partnership..................201

5.5.3 Theme 3. Level of gaming intentionality: Influences............................205

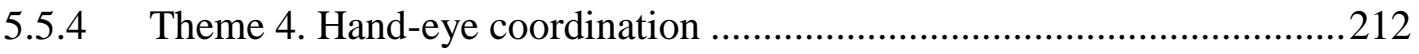

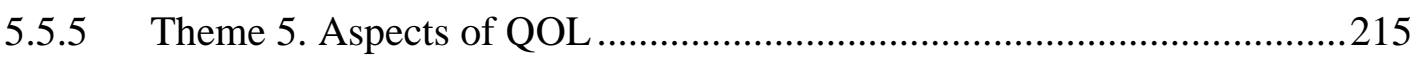

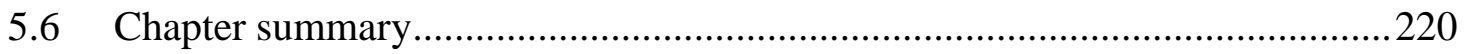

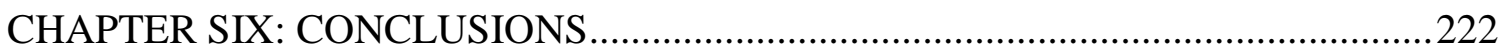

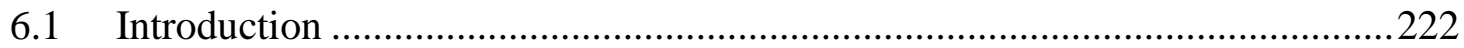

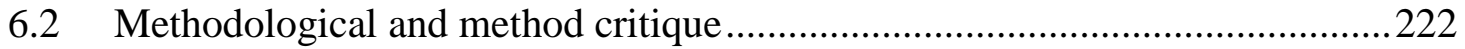

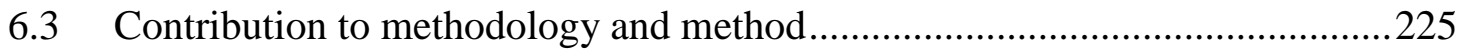

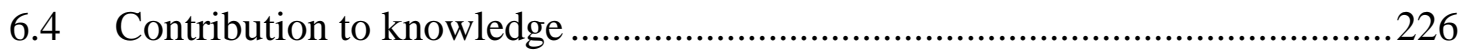

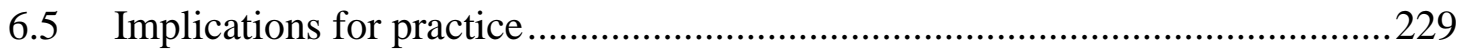

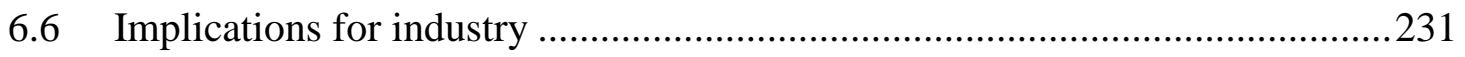

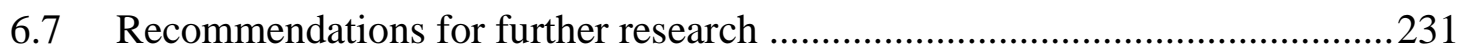

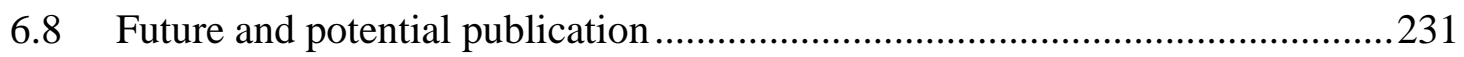

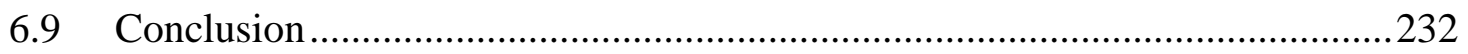

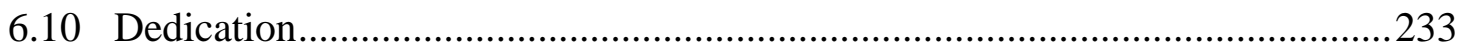

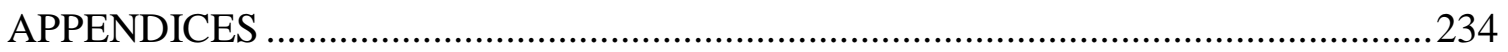

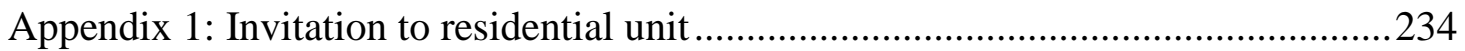

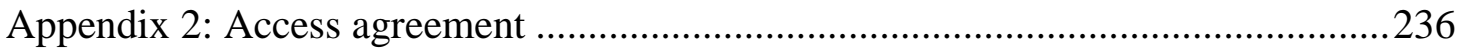

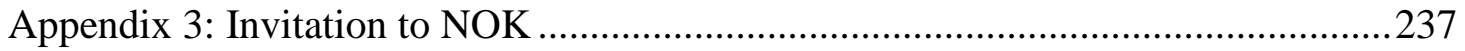

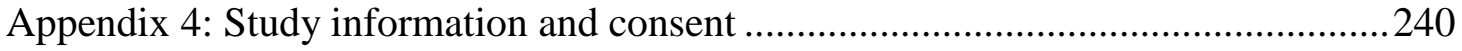

Appendix 5: Simplified information sheet for PWD .............................................24

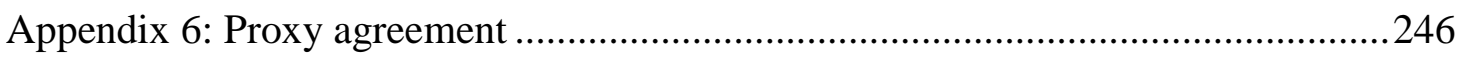




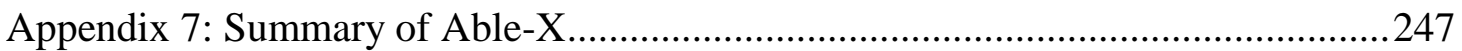

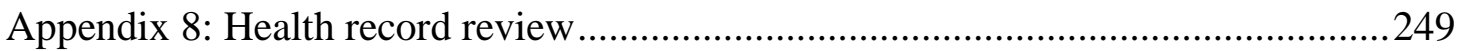

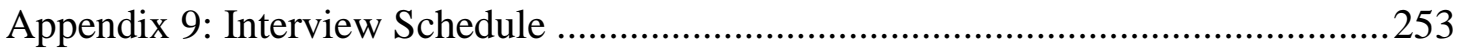

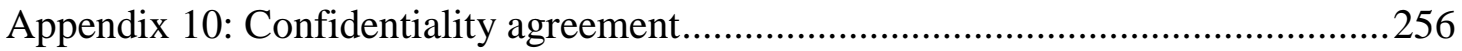

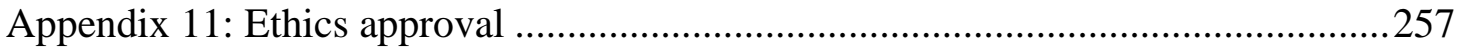

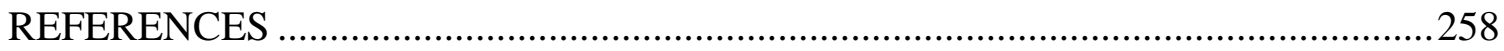




\section{LIST OF TABLES}

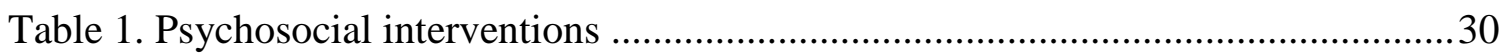

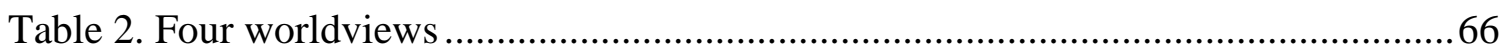

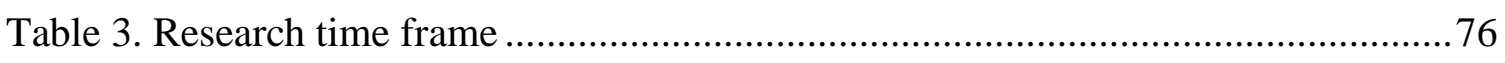

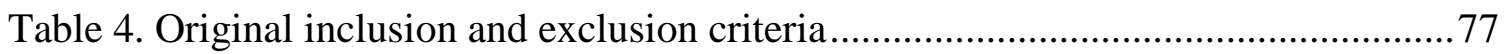

Table 5. Amended and final inclusion and exclusion criteria ....................................... 78

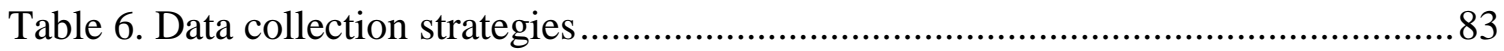

Table 7. Summary of data collection from all three sites ................................................ 88

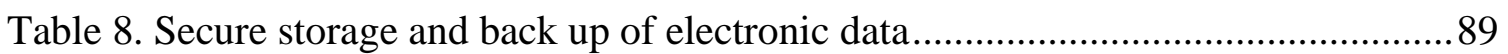

Table 9. Example of raw data meaning unit to sub-theme of BPSD .............................. 93

Table 10. Procedure for exergaming with Able-X-initiation and progress ...................... 98

Table 11. Joy: Summary of baseline and end-point measures...................................... 113

Table 12. Joy: Quality of life points change pre-and post-gaming...............................114

Table 13. Iris: Summary of baseline and end-point measures.................................... 121

Table 14. Iris: Quality of life points change pre-and post-gaming ................................ 122

Table 15. Bill: Summary of baseline and end-point measures ..................................... 129

Table 16. Bill: Quality of life points change pre-and post-gaming ................................ 130

Table 17. Thomas: Summary of baseline and end-point measures ................................ 137

Table 18. Thomas: Quality of life points change pre-and post-gaming ......................... 138

Table 19. Maya: Summary of baseline and end-point measures .................................. 145

Table 20. Maya: Quality of life points change pre-and post-gaming ............................ 146

Table 21. Risa: Summary of baseline and end-point measures ................................... 153

Table 22. Risa: Quality of life points change pre-and post-gaming .............................. 154

Table 23. Clara: Summary of baseline and end-point measures ................................... 161

Table 24. Clara: Quality of life points change pre-and post-gaming ............................ 162

Table 25. Alfie: Summary of baseline and end-point measures ..................................... 169

Table 26. Alfie: Quality of life points change pre-and post-gaming ............................ 170

Table 27. Alana: Summary of baseline and end-point measures................................... 177

Table 28. Alana: Quality of life points change pre-and post-gaming............................. 179

Table 29. Florence: Summary of baseline and end-point measures .............................. 187

Table 30. Florence: Quality of life points change pre-and post-gaming ........................ 188

Table 31. Demographic and personal characteristics of cases .................................... 195

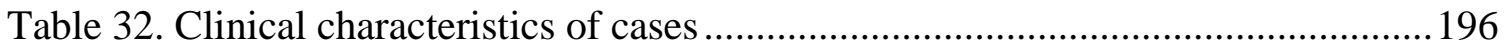

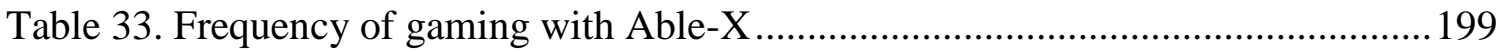




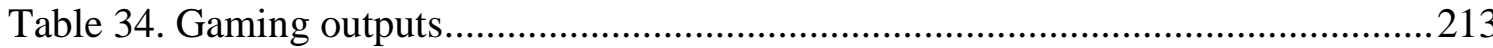

Table 35. Summary of findings from the QOL-AD outcome measure ..........................2. 216

Table 36. Summary of findings from the CSDD and RAID outcome measures............217

\section{LIST OF FIGURES}

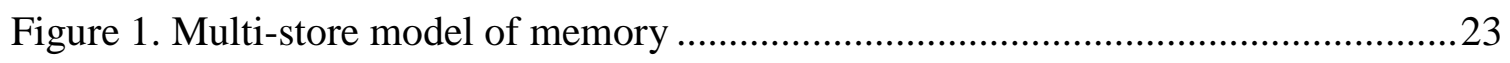

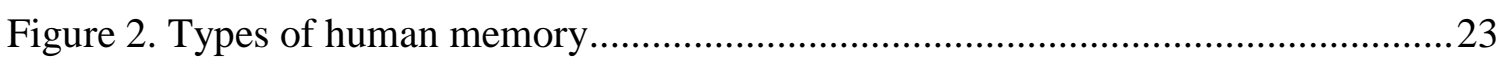

Figure 3. The role of explicit and implicit memory in relation to a basic task .................24

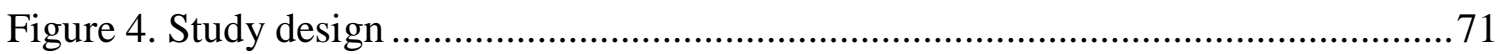

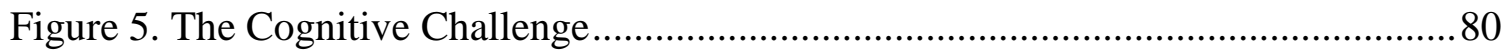

Figure 6. Seven steps from data corpus to theme …...................................................... 91

Figure 7. Data sorting: Logical data corpus sets....................................................... 92

Figure 8. Joy: Computer-generated exergaming outputs............................................ 112

Figure 9. Iris: Computer-generated exergaming outputs ............................................. 120

Figure 10. Bill: Computer-generated exergaming outputs ........................................ 128

Figure 11. Thomas: Computer-generated exergaming outputs .................................... 136

Figure 12. Maya: Computer-generated exergaming outputs ........................................ 144

Figure 13. Risa: Computer-generated exergaming outputs ........................................ 152

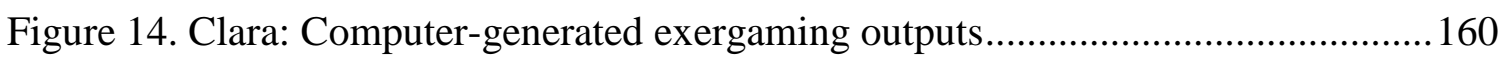

Figure 15. Alfie: Computer-generated exergaming outputs ......................................... 168

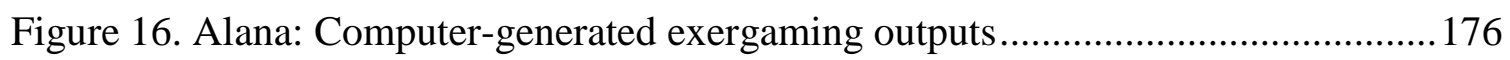

Figure 17. Florence: Computer-generated exergaming outputs .................................. 185 


\section{GLOSSARY OF ABBREVIATIONS}

\begin{tabular}{|l|l|}
\hline AChE & Acetylcholinesterase \\
\hline AD & Alzheimer's disease \\
\hline ADLs & Activities of daily living \\
\hline BPSD & $\begin{array}{l}\text { Behavioural and psychological symptoms of dementia (sometimes called } \\
\text { non-cognitive symptoms of dementia) }\end{array}$ \\
\hline CDR & Clinical Dementia Rating \\
\hline CS & Case study \\
\hline CSDD & $\begin{array}{l}\text { Cornell Scale for Depression in Dementia (Alexopoulos, Abrams, Young, } \\
\text { \& Shamoian, 1988). }\end{array}$ \\
\hline CST & Cognitive stimulation therapy \\
\hline DT & Diversional Therapist \\
\hline EL & Errorless learning \\
\hline EPA & Enduring Power of Attorney \\
\hline FAST & Functional Assessment Staging Test \\
\hline FN & Field note \\
\hline GDS & Global Deterioration Scale \\
\hline HCA & Health Care Assistant \\
\hline HDEC & Health and Disability Ethics Committee \\
\hline IADLs & Instrumental activities of daily living \\
\hline MMSE & Mini-Mental State Examination (Folstein, Folstein, \& McHugh, 1975), \\
\hline MMSE-2:BV & $\begin{array}{l}\text { Mini-Mental State Examination 2: Brief version (Folstein, Folstein, White, } \\
\text { \& Messer, 2010). }\end{array}$ \\
\hline NCSD & Non-cognitive symptoms of dementia (sometimes called BPSD) \\
\hline NOK & Next of Kin \\
\hline NZ & New Zealand \\
\hline PCC & Person-centred care \\
\hline PWD & Person/people with dementia \\
\hline PRN & Pro re nata (as the circumstance arises) \\
\hline QOL & Quality of life \\
\hline QOL-AD & Quality of Life -AD (Logsdon, 1996). \\
\hline RAID & $\begin{array}{l}\text { Rating Anxiety in Dementia Scale (Shankar, Walker, Frost, \& Orrell, } \\
\text { 1999). }\end{array}$ \\
\hline TA & Thematic analysis \\
\hline VaD & Vascular dementia \\
\hline VL & Video live \\
\hline VN & Video note \\
\hline
\end{tabular}




\section{GLOSSARY OF TERMS}

\begin{tabular}{|c|c|}
\hline $\begin{array}{l}\text { Alzheimer's Disease } \\
\text { (AD) }\end{array}$ & $\begin{array}{l}\text { AD is the most common dementia, accounting for } 60-80 \% \text { of } \\
\text { cases. It is slow and progressive and presents with decline of } \\
\text { memory and cognitive ability, accompanied by loss of skill. } \\
\text { Early symptoms include difficulty remembering names and } \\
\text { recent events. Later symptoms include impaired judgment, } \\
\text { confusion, disorientation and behaviour changes (Alzheimer's } \\
\text { Association, 2013a,b; Brickell, 2012). }\end{array}$ \\
\hline $\begin{array}{l}\text { Activities of daily living } \\
\text { (ADLs) }\end{array}$ & $\begin{array}{l}\text { Activities of daily living includes; eating, toileting, dressing, } \\
\text { bathing or showering, getting in or out of bed or chairs and } \\
\text { walking (Stineman et al., 2014, p. 979). }\end{array}$ \\
\hline Agent & Intentionally active. \\
\hline Amitriptyline & $\begin{array}{l}\text { A tricyclic antidepressant medication (Medsafe, 2014c). This } \\
\text { agent has significant anticholinergic activity, can cause } \\
\text { hypotension, and delayed cardiac conduction (not first line in } \\
\text { dementia) (Alzheimer's Association, 2015b; Small et al., } \\
\text { 1997). }\end{array}$ \\
\hline Citalopram & $\begin{array}{l}\text { An antidepressant medication for the treatment of low mood } \\
\text { and irritability (Medsafe, 2014e). }\end{array}$ \\
\hline $\begin{array}{l}\text { Cornell Scale for } \\
\text { Depression in Dementia } \\
\text { (CSDD) }\end{array}$ & $\begin{array}{l}\text { A 19-item scale ranging from } 0 \text { to } 38 \text {. A total score of } 8 \text { or more } \\
\text { suggests significant depressive symptoms (Alexopoulos, } \\
\text { Abrams, Young, \& Shamoian, 1988). }\end{array}$ \\
\hline Diazepam & $\begin{array}{l}\text { A benzodiazepine medication used to treat agitation. Should be } \\
\text { avoided in dementia as can cause confusion, impaired } \\
\text { cognition, and sedation }\left(\mathrm{Bpac}^{\mathrm{NZ}}, 2008\right) \text {. }\end{array}$ \\
\hline Donepezil & $\begin{array}{l}\text { A cholinesterase inhibitor, which provides some symptomatic } \\
\text { relief by slowing down cognitive decline and loss of } \\
\text { independence }\left(\mathrm{Bpac}^{\mathrm{NZ}}, 2010 ; \text { Medsafe, 2014a). }\right.\end{array}$ \\
\hline $\begin{array}{l}\text { Drift } \\
\text { (associated with the } \\
\text { controller) }\end{array}$ & $\begin{array}{l}\text { Relates to the use of Able-X and refers to the drift of the } \\
\text { controller from the centre of the gaming screen and the } \\
\text { associated adjustment that the player had to make to be in } \\
\text { control of the on-screen representation. For example, the } \\
\text { player may be pointing the controller to the centre screen, but } \\
\text { the on-screen representation of the hand-held controller may be } \\
\text { several centimetres off the centre point, requiring the player to } \\
\text { move the controller until the on-screen representation is in the } \\
\text { centre spot, or press a button to re-centre the controller. }\end{array}$ \\
\hline Dysphagia & $\begin{array}{l}\text { A swallowing disorder, which occurs mainly in older people } \\
\text { and in, advanced dementia (Ekberg, Hamdy, Woisard, Wuttge- } \\
\text { Hannig \& Ortega, 2002). }\end{array}$ \\
\hline Error starer-Error inertia & $\begin{array}{l}\text { A novel concept identified through this research, which I } \\
\text { describe as 'gestural startled effect that momentarily disrupted } \\
\text { gaming and appeared to trigger gaming inertia, which was } \\
\text { generally followed by a run of errors or a term I coined as a } \\
\text { result of the research, 'error starer-error inertia' moments, the } \\
\text { time period between 'startled' and 'refocused', where gaming } \\
\text { momentarily ceased". }\end{array}$ \\
\hline
\end{tabular}




\begin{tabular}{|c|c|}
\hline Exergaming & $\begin{array}{l}\text { Exergaming is defined as "technology-driven physical } \\
\text { activities, such as... [computer gaming], that requires } \\
\text { participants to be physically active or exercise in order to play } \\
\text { the game" (American College of Sports Medicine, 2013, p. 1). }\end{array}$ \\
\hline Expressive dysphasia: & $\begin{array}{l}\text { The person understands the question, or conversation, but their } \\
\text { response in non-fluent } \\
\text { (http://www.nle.nottingham.ac.uk/websites/stroke/contents/exp } \\
\text { dysphasia.html). }\end{array}$ \\
\hline $\begin{array}{l}\text { Functional Assessment } \\
\text { Staging Test (FAST) }\end{array}$ & $\begin{array}{l}\text { Describes a continuum of seven successive stages and sub- } \\
\text { stages from normality to most severe dementia of Alzheimer } \\
\text { Disease (AD) type. The stages are "enumerated to be... } \\
\text { concordant with the GDS stages from where they were } \\
\text { derived" (Auer \& Reisberg, 1997, p. 168; Reisberg, 1988). } \\
\text { 1. No difficulty } \\
\text { 2. Subjective work difficulties } \\
\text { 3. Decreased organizational capacity } \\
\text { 4. Decreased ability to perform complex tasks } \\
\text { 5. Requires assistance choosing proper clothing } \\
\text { 6. a) Improperly putting on clothes without assistance or } \\
\text { cuing } \\
\text { b) Unable to bath due to difficulty adjusting water } \\
\text { temperature } \\
\text { c) Inability to handle mechanics of toileting } \\
\text { (flush/wipe) } \\
\text { d) Urinary incontinence } \\
\text { e) Faecal incontinence } \\
\text { 7. a) Ability to speak limited to half a dozen words or } \\
\text { fewer } \\
\text { b) Speech ability limited to a single intelligible word } \\
\text { c) Ambulatory ability is lost (needs assistance) } \\
\text { d) Cannot sit up without assistance } \\
\text { e) Loss of ability to smile } \\
\text { f) Loss of ability to hold head up independently }\end{array}$ \\
\hline Frontotemporal dementia & $\begin{array}{l}\text { Also known as Pick's disease; is a group of disorders rather } \\
\text { than a single disease and accounts for 5-10\% of dementias. } \\
\text { Early symptoms include changes in personality and behaviour } \\
\text { such as disinhibition, risk taking, neglect of personal hygiene } \\
\text { or dressing oddly. Also, the person may have changes in } \\
\text { emotion, speech and language which occur despite other } \\
\text { cognitive functions remaining initially unchanged (Alzheimer's } \\
\text { Association, 2013b; Perkins, 2013). }\end{array}$ \\
\hline Game able & $\begin{array}{l}\text { A novel concept identified through this research. Participants } \\
\text { play games with independent agency, with rare or minimal } \\
\text { assistance. }\end{array}$ \\
\hline Game enabled & $\begin{array}{l}\text { A novel concept identified through this research. Participants } \\
\text { follow verbal instructions contemporaneously-every 5-10 } \\
\text { seconds, to remain gaming active. }\end{array}$ \\
\hline
\end{tabular}




\begin{tabular}{|c|c|}
\hline Game shy & $\begin{array}{l}\text { A novel concept identified through this research. Participants } \\
\text { are unable to self-initiate gaming without assistance, resulting } \\
\text { in gaming inertia. Participants required reintroduction to the } \\
\text { game at every session and before each game. }\end{array}$ \\
\hline Gaming by association & $\begin{array}{l}\text { A novel concept identified through this research. An engaged } \\
\text { observer enjoying the success of other gamers during small } \\
\text { group gaming. }\end{array}$ \\
\hline $\begin{array}{l}\text { Global deterioration } \\
\text { Scale (GDS) }\end{array}$ & $\begin{array}{l}\text { Auer and Reisberg (1997, pp. 167-168), describes seven } \\
\text { distinguishable global stages from normality to severe } \\
\text { dementia of AD type. The seven GDS stages are: } \\
\text { 1. Individuals are free from...subjective cognitive } \\
\text { complaints and clinically manifest deficits; } \\
\text { 2. Individuals have... subjective cognitive complaints; } \\
\text { 3. Individuals have...subtle deficits that are manifest in a } \\
\text { detailed clinical interview... deficits...insufficient... to } \\
\text { interfere with either basic or instrumental activities of } \\
\text { daily life, although executive functions are frequently } \\
\text { compromised; } \\
\text { 4. Individuals generally have deficit in management of } \\
\text { instrumental activities of daily life; } \\
\text { 5. Individuals can no longer survive if left on their own } \\
\text { 6. Individuals lose basic activity of daily life capacities... } \\
\text { behaviour disturbances peak in occurrence and } \\
\text { severity; and } \\
\text { 7. Individuals require continuous assistance for survival. }\end{array}$ \\
\hline $\begin{array}{l}\text { Instrumental Activities of } \\
\text { Daily Living } \\
\text { (IADLs) }\end{array}$ & $\begin{array}{l}\text { Instrumental Activities of Daily Living include using the } \\
\text { telephone, managing money (banking), preparing his/her own } \\
\text { meals (using oven and storing food in fridge/freezer), light } \\
\text { housework (vacuum cleaner), shopping for personal items, and } \\
\text { heavy housework (Stineman et al., 2014, p. 979). }\end{array}$ \\
\hline Lorazepam & $\begin{array}{l}\text { An anxiolytic medication used to treat anxiety, disruptive } \\
\text { behaviour, and resistance (Medsafe, 2015c). }\end{array}$ \\
\hline Nonagenarian & A person in his or her nineties. \\
\hline $\begin{array}{l}\text { Non-cognitive symptoms } \\
\text { of dementia }\end{array}$ & $\begin{array}{l}\text { Includes symptoms of disturbed perception, thought content, } \\
\text { mood or behaviour; frequently occurring in patients with } \\
\text { dementia and are sometimes referred to as behavioural and } \\
\left.\text { psychological symptoms of dementia (BPSD) (Bpac }{ }^{\mathrm{nz}}, 2013\right) \text {. }\end{array}$ \\
\hline Octogenarian & A person in his/her eighties. \\
\hline Paroxetine & $\begin{array}{l}\text { An antidepressant medication for low mood, irritability } \\
\text { (Alzheimer's Association, 2015a), social anxiety disorders, and } \\
\text { depression (Medsafe, 2014b). Affects chemicals in the brain } \\
\text { that may become unbalanced. }\end{array}$ \\
\hline Prochlorperazine maleate & $\begin{array}{l}\text { An antipsychotic medication that is also used to treat vertigo. } \\
\text { It has antiemetic and antipsychotic activity with little sedative } \\
\text { action (Medsafe, 2014d). Antipsychotic medications are } \\
\text { associated with an increased risk of stroke and death in older } \\
\text { people with dementia and should only be used with extreme } \\
\text { caution (Alzheimer's Association, 2015a). }\end{array}$ \\
\hline
\end{tabular}




\begin{tabular}{|c|c|}
\hline Proxy & $\begin{array}{l}\text { The agency, function, or office of a deputy who acts as a } \\
\text { substitute for another http://www.merriam- } \\
\text { webster.com/dictionary/proxy }\end{array}$ \\
\hline $\begin{array}{l}\text { Quality of Life - } \\
\text { Alzheimers Disease } \\
\text { (QOL-AD) }\end{array}$ & $\begin{array}{l}\text { A 13-item scale, Score 13-52. The higher the score the higher } \\
\text { the assessed/perceived QOL. Need to establish marital } \\
\text { status/closest relationship (for q7) in advance. Surrogate } \\
\text { completes a self-administered questionnaire in the presence of } \\
\text { the researcher so the researcher can answer any questions. } \\
\text { Researcher completes interview with the person with dementia } \\
\text { who should usually have an MMSE of } 10 \text { or above (Logsdon, } \\
\text { 1996). }\end{array}$ \\
\hline Quetiapine & $\begin{array}{l}\text { An antipsychotic medication commonly used to treat } \\
\text { behavioural and psychiatric symptoms of Alzheimer's disease. } \\
\text { Antipsychotic medications are associated with an increased } \\
\text { risk of stroke and death in older people with dementia and } \\
\text { should only be used with extreme caution (Bpac }{ }^{\text {nz }}, 2013 \text {; } \\
\text { Medsafe 2015b). }\end{array}$ \\
\hline $\begin{array}{l}\text { Rating Scale for Anxiety } \\
\text { in Dementia } \\
\text { (RAID) }\end{array}$ & $\begin{array}{l}18 \text { item scale. Score is sum of items 1-18 (0-54). A score of } 11 \\
\text { or more suggest significant clinical anxiety. Signs and } \\
\text { symptoms over the last } 2 \text { weeks only. Symptoms that arise due } \\
\text { to physical illness or medication are excluded from scoring. } \\
\text { Clinician/researcher determines final score (Shankar, Walker, } \\
\text { Frost, \& Orrell, 1999). }\end{array}$ \\
\hline $\begin{array}{l}\text { Repeated success of easy } \\
\text { gaming }\end{array}$ & $\begin{array}{l}\text { A novel concept identified through this research. Participants } \\
\text { preferred continued repetitive gaming at the most basic gaming } \\
\text { level, rather than the risk of not being successful at a more } \\
\text { challenging level. }\end{array}$ \\
\hline Risperidone & $\begin{array}{l}\text { An antipsychotic medication used to treat some of the } \\
\text { behavioural and psychological symptoms of dementia }\left(\mathrm{Bpac}^{\mathrm{nz}} \text {, }\right. \\
\text { 2013, p.54; Medsafe, 2015a). Antipsychotic medications are } \\
\text { associated with an increased risk of stroke and death in older } \\
\text { people with dementia and should only be used with extreme } \\
\left.\text { caution (Bpac }{ }^{\mathrm{nz}}, 2013\right) \text {. }\end{array}$ \\
\hline Septuagenarian & A person in his/her seventies. \\
\hline Sexagenarian & A person in his/her sixties. \\
\hline $\begin{array}{l}\text { Tetrachotomy/ } \\
\text { Tetrachotomous }\end{array}$ & $\begin{array}{l}\text { The equivalent Greek adverb for the number four is tetracha } \\
\text { (divided into four parts, fourfold), and the resulting term } \\
\text { following this pattern is tetrachotomy (Huber, 2003). Regularly } \\
\text { dividing by four; having quadruple arrangements } \\
\text { (http://www.merriam-webster.com/dictionary/tetrachotomous). }\end{array}$ \\
\hline Vascular dementia (VaD) & $\begin{array}{l}\text { Occurs after a stroke, previously known as multi-infarct } \\
\text { dementia or post-stroke dementia. It is the second most } \\
\text { common cause of dementia (10-20\%) and is likely to present } \\
\text { with impaired judgment or impaired ability to plan steps } \\
\text { needed to complete a task (executive function), as opposed to } \\
\text { the memory loss often associated with AD (Alzheimer's } \\
\text { Association, 2013b). Vascular dementia has a much more } \\
\text { varied presentation than AD, depending on where the vascular } \\
\text { damage has occurred. For example, if the parietal lobe is } \\
\text { damaged the person may have problems with numbers or }\end{array}$ \\
\hline
\end{tabular}




\begin{tabular}{|l|l|}
\hline Whānau & $\begin{array}{l}\text { recognising things, whereas if the frontal lobe is damaged the } \\
\text { person may have problems making decisions (Perkins, 2013) }\end{array}$ \\
\hline $\begin{array}{l}\text { Whānau is an extended family group spanning three or four } \\
\text { generations, and is the basic unit of Māori society } \\
\text { (http://www.teara.govt.nz/en/tribal-organisation/page-4). }\end{array}$ \\
\hline
\end{tabular}




\section{CHAPTER ONE: INTRODUCTION AND BACKGROUND LITERATURE}

\subsection{Introduction to the thesis}

The greatest impact of dementia is on the quality of life (QOL) of those living with the disease and that of their families and caregivers (Bpac ${ }^{\mathrm{nz}}, 2013$; Perkins, 2013; Prince, Albanese, Guerchet, \& Prina, 2014; Snowden, Sato, \& Roy-Byrne, 2003). Further, dementia care and resource utilisation pose considerable challenges, including care burden and economic impact at all levels of healthcare in New Zealand and globally (Alzheimer's New Zealand, 2012; Prince et al., 2015). One of the primary challenges of dementia care is the provision of pharmacological and non-pharmacological interventions that have been proven to have beneficial effect in delaying cognitive and functional decline in people with dementia (PWD), empowering them to remain socialised and engaged in life and independent living (Birks, 2006; McShane, Areosa Sastre, \& Minakaran, 2006; NICESCIE, 2006/2015; Rodda \& Carter, 2012). Over the last three decades, numerous nonpharmacological, psychosocial cognitive oriented interventions have evolved (Brickell, 2012) and some demonstrate significant improvement in both cognition and QOL in this population (Woods, Aguirre, Spector, \& Orrell, 2012). There is also early evidence of improvement in physical functioning (Ballard, Khan, Clack, \& Corbett, 2011). The strongest evidence is for cognitive stimulation, cognitive stimulation therapy (CST), and cognitive rehabilitation (Ballard et al., 2011; Woods et al., 2012). However, traditional cognitive oriented interventions tend to be labour-intensive, and underutilised in everyday care (George \& Whitehouse, 2011; Woods et al., 2012). A more contemporary focus on utilising computer interventions to preserve cognitive and social functioning is emerging in the literature (Gelfand et al., 2014), however, the effects of computer-assisted exergaming $^{1}$ on QOL, including physical functioning in PWD is relatively unknown and yet to be determined. Therefore, research into cognitive stimulation as a psychosocial intervention (PSI), utilising computer-assisted exergaming technology (Able-X) ${ }^{2}$ for PWD will contribute new knowledge in this field.

\footnotetext{
${ }^{1}$ Exergaming is defined as "technology-driven physical activities, such as [computer gaming], that requires participants to be physically active or exercise in order to play the game" (American College of Sports Medicine, 2013, p. 1).

${ }^{2}$ Now branded as ableX.
} 
This thesis documents my exploration of the social occupation of computerassisted exergaming with Able-X in an effort to answer the research question: How does Able-X affect QOL, cognitive functioning and physical functioning in people $\geq 65$ years, who are living with dementia and residing in assisted care settings in NZ? This first chapter presents an introduction to the thesis and a short reflection on my research interests, reasons, and justification for undertaking this study. The study is positioned as an exploration of the potential benefits of social occupation of computer-assisted exergaming with Able-X, in partnership with PWD, with a primary focus of QOL. I present background literature, including elements of my literature review, to position and contextualise the thesis. The background literature begins with an overview of global and national dementia demographics and prevalence. I then define dementia and critically explore diagnosis and the clinical presentation of dementia, with a focus on the five most common dementia subtypes, three of which are relevant to this study. A critical overview of current pharmacological treatments is outlined, including their limitations in terms of dementia type, following which the global disease care burden, economic impact, and risk factors for developing dementia are critically explored. I then present the ontology and epistemology of Descartes (1993; 1997), in relation to thought, thinking, mind and body, and the impact of this epistemology on PWD. This leads to a review of the stigma literature. Understanding the dementia process requires a general knowledge about how the human memory works, so a brief overview is provided. The chapter concludes with a statement about how QOL is conceptualised within this thesis.

\subsection{Research interest from a professional perspective}

For many years, I have had an interest in, and been involved in research. In 2012, I accepted a position as Research Fellow to Dr Kay de Vries, a Senior Lecturer at the Graduate School of Nursing Midwifery and Health, at Victoria University of Wellington, NZ. Dr de Vries' research interests include, but are not limited to, dementia care and endof-life care ( Brooker, La Fontaine, de Vries, Latham, 2013; Brooker, La Fontaine, de Vries, Porter, \& Surr, 2011; de Vries, Brooker, \& Smith, 2012; de Vries, DruryRuddlesden, \& Gaul, 2016; de Vries, La Fontaine, \& Brooker, 2010).

We worked together on three concurrent case study (CS) projects. The first study examined the experiences of older PWD and their family/whānau, caregivers and staff 
during admission to an acute hospital ward (de Vries et al., 2016). On reflection, I was challenged by the balance of the focus of care, which was weighted in favour of the acute admission diagnosis at the expense of a potentially deteriorating acute on chronic dementia state. It was also apparent that beneficial and purposeful occupation for PWD in the acute care sector was negligible and a repetitive story of episodes of non-cognitive behavioural problems that were dealt with through a 'watching service', rather than providing a meaningful occupation such as psychosocial interventions (PSI). A watching service, sometimes called supervision, was provided by staff or on occasions by family/whānau and sometimes in partnership. Moreover, there was emerging evidence of security staff being called to assist in the management of a person with dementia (PWD) if behavioural and psychological symptoms of dementia (BPSD) were beyond the scope of the watcher service (de Vries et al., 2016). There is the potential for PWD to be marginalized in all care settings, and I was curious to understand what could be done to improve the QOL for people journeying with dementia through meaningful and purposeful occupation.

In the second and third research projects (publications pending), I was tasked with interviewing and recording stories about acute hospital admission and end of life care experience of PWD, narrated by their next-of-kin (NOK). Most of the stories revealed glimpses of a lack of focus on the wider concepts of QOL and quality of end-of-life pathways and all revealed variability in terms of access to appropriate, beneficial and purposeful occupation, reiterating the importance of being meaningfully occupied, and to have and maintain meaningful and purposeful bodily agency throughout life course. My experience of working with Dr de Vries from 2012 to the present day has undoubtedly shaped me and my research interests and motives, which were inspired by the many touching and moving narratives, which acknowledge that we all need to do more to make a difference in the lives of people who are living with and dying with dementia.

In early 2013, Dr de Vries was contacted by a representative from Im-Able $\operatorname{Ltd}^{3}$., a privately owned NZ company, with a view to conducting joint clinical research using the Able-X, which had recently been developed for the rehabilitation of stroke survivors (Jordan, Sampson \& King, 2014; King et al., 2010; King, Hijmans, Sampson, Satherley, \& Hale, 2012; Sampson, Shau, \& King, 2012), and commercialised as an exercise system;

\footnotetext{
${ }^{3}$ Now called ableX healthcare Ltd.
} 
a hand-held controller allows the user to undertake an exercise programme on a computer (Im-Able Ltd., 2013). The Able-X exercise rehabilitation system is based on repetition of goal directed exercises, and the theory of neuroplasticity, which allows the brain to form new synaptic connections to take over functions of other parts of the brain that may be damaged (Hallett, 2001; King et al., 2010; Prince et al., 2014). Integral to this theory is "experience-dependent neural plasticity", which is based on the notion that environmental input can influence the generation of new brain connections (Gelfand, Vick \& Lewis, 2014, p. 55). The Able-X can be used for bilateral or unilateral arm exercise, and has two gaming options: a customised gaming routine that prompts and leads the user through an exercise routine based on their level of capability, and a prescribed routine that allows health professionals to design the exercise session based on the abilities and preferences of the user.

In early 2013, the device was being used by a resident with dementia in a small residential/dementia unit in North Island NZ, and a staff member reported that the resident, appeared to get great pleasure and enjoyments from playing the games (Vather ${ }^{4}$, personal communication February 2013). This feedback was the stimulus for the company representative to contact Victoria University. The Able-X has been recognised with four awards in Australia and NZ (Im-Able Ltd., 2013), and there was interest in exploring if it could deliver functional and QOL improvements for PWD, as the device had not yet been explored with this population group.

In 2013, a jointly developed proposal was submitted to the Callaghan Innovation Fund, NZ, resulting in me being awarded the Callaghan Innovation Scholarship 2014, established under the Callaghan Innovation Act 2012. Conflict of interest and intellectual property issues were addressed through a 'contract for educational fellowship'; the signatories were Callaghan Innovation, Im-Able Ltd., Victoria University of Wellington, and myself. The obligations of all parties were clearly documented, for me this including not being employed by, or having a relationship with any other parties, other than to undertake the programme of study as documented in the schedule. Involvement of the company mentor was limited to providing me with equipment (Able-X and gaming suite) and reading and signing quarterly reports for the Ministry of Business, Innovation and

\footnotetext{
${ }^{4}$ Vather, from Im-Able Ltd., a privately owned NZ company.
} 
Employment NZ. Additional reflexivity is presented in section 3.3.6.2. In the following section, I present an overview of dementia demographics and prevalence rates.

\subsection{Dementia demographics and prevalence}

Dementia is a condition that is unbounded by socioeconomic factors and has an extensive and devastating societal and familial reach (Alzheimers New Zealand, 2012). In the late 1990s Kitwood (1997) predicted that dementia would present as the most momentous epidemiological challenge of the $20^{\text {th }}$ century and that all aspects of life as we know it would be touched in some way or another. In the $21^{\text {st }}$ century the economic, political and social impact of dementia are a major concern for healthcare funders and providers (Alzheimers New Zealand, 2012; Alzheimers New Zealand Incorporated, 2008; The Lancet Neurology, 2014). Dementia is NZ's biggest looming health crisis and follows a global upward trend, although when compared to 2009 data prevalence estimates have decreased in Europe and the Americas, but increased in Asia and Africa (Prince et al., 2015). New Zealand has an ageing population and as the population ages the prevalence of dementia increases, however dementia is not solely related to chronological age, but also to the wider socioeconomic determinants of health (Dyall, 2014).

Globally, more than 35 million people had dementia in 2010 and this number is expected to exceed 115 million by 2050 (The Lancet Neurology, 2014; van den Berg, \& Splaine, 2012). In 2008 there were 40,746 people diagnosed with dementia in NZ; three years later this number had reached $48,182,1.1 \%$ of the total population, an increase of over 18\%. By 2026, it is estimated there will be 74,821 PWD in NZ and by 2050 this number is estimated to be 147,359 , accounting for $2.6 \%$ of the population, of which 87,145 (59\%) will be female and 60,214 (41\%) will be male. This follows the worldwide upward prevalence trend that is estimated to be doubling every 20 years (Alzheimers New Zealand, 2012; Alzheimers New Zealand Incorporated, 2008; Alzheimer's Research Trust, 2010). However, Prince et al. (2015) are sceptical of the validity of the current evidence base for prevalence data, due to evidence of decreased prevalence research efforts in high-income countries since the 1990s. A trend that is ongoing, resulting in increasingly out of date prevalence figures. 
The prevalence and incidence of dementia is expected to be compounded by a number of factors. The generation of baby boomers is reaching an age where they are susceptible to neurodegenerative disorders (Choi \& Twamley, 2013). Moreover, advanced disease surveillance, early screening for dementia, along with increased longevity, and increased numbers of people living with a comorbid life-limiting illness may predispose to higher prevalence (Choi \& Twamley, 2013; Whitmer, Gunderson, Quesenberry, Zhou, $\&$ Yaffe, 2007). In addition, due to demographic changes in NZ, increasing numbers of PWD will be from non-European backgrounds, including Asian (8.4\%), Māori (5.7\%), and Pacific peoples (2.6\%) (Alzheimers New Zealand, 2012). Māori, the tāngata whenua (indigenous people) of Aotearoa (NZ) and Pacific peoples are particularly at risk due to wider socioeconomic determinants of health, resulting in higher prevalence of chronic, and comorbid disease. This reduces their life expectancy by eight to ten years (for Māori) and six to seven years (for Pacific peoples) and increases their risk for developing dementia (Alzheimers New Zealand, 2012; Dyall, 2014).

\subsection{Defining and diagnosing dementia}

Dementia is a term that describes a group of more than 200 syndromes characterised by chronic and progressing illnesses that affect the brain and cause decline in mental ability severe enough to interfere with activities of daily life (Alzheimers New Zealand Incorporated, 2008; Stephan \& Brayne, 2014; Perkins, 2013). These chronic and terminal syndromes affect higher cortical functions: memory, thinking, orientation, comprehension, calculation, learning capacity, language and judgment, which is often preceded by global deterioration of social, physical and emotional states (Prince et al., 2014).

Diagnosing dementia requires time to conduct a series of comprehensive tests and timely access to a variety of investigations (Brickell, 2012). However, there is a reluctance in primary care to be directly involved in dementia diagnosis (Banerjee \& Wittenberg, 2009; Iliffe \& Manthorpe, 2004; Iliffe, Wilcock \& Haworth, 2006), with concerns about competency, lack of experience and confidence in making and disclosing a diagnosis often being cited, along with inadequate access to appropriate resources (Iliffe, Jain, \& Wilcock, 2009; Iliffe, Manthorpe, \& Eden, 2003; Iliffe, \& Wilcock, 2005; Iliffe et al., 2006). Therefore, in line with global diagnostic rates, up to $60 \%$ of people with dementia in NZ 
do not receive a diagnosis until late in the course of the disease, if at all (Brickell, 2012; Iliffe et al., 2003). Bamford, Eccles, Steen, and Robinson (2007) found differences in consultation patterns with GP service providers of up to four years preceding the diagnosis of dementia. Following diagnosis of dementia a person's life expectancy is estimated to be 7-12 years (Banerjee \& Wittenberg, 2009).

A global consensus of dementia epidemiologists estimate a new person is diagnosed with dementia every seven seconds (Boustani et al., 2008). Even so, the actual number of PWD remains underestimated in NZ and globally by up to 50\% (Alzheimers New Zealand Incorporated, 2008; Brickell, 2012; Iliffe, \& Manthorpe, 2004; Prince, Bryce, \& Ferri, 2011; The Lancet Neurology, 2014). Contributory factors for delayed diagnosis include denial among family members (Connell, Roberts, McLaughlin, \& Carpenter, 2009; Teel 2004), and not valuing or seeking early screening for dementia for a variety of reasons, including the stigma associated with a dementia diagnosis (Boustani et al., 2011; Boustani, Watson, Fultz, Perkins, \& Druckenbrod, 2003a; Iliffe et al., 2003; Iliffe, \& Orrell, 2006). Conversely, screening and diagnostic stigma did not seem to be an attitudinal concern for caregivers and non-caregivers in a cross-sectional study in the USA (Boustani et al., 2011), concerns raised were about not being taken seriously and whether their employer or health insurer would find out about their diagnosis. In another USAbased study, Connell et al. (2009) found that family members strongly endorsed the benefits of early screening for early diagnosis. Also, the majority of family member respondents did not endorse any of the commonly suggested stigmatising barriers (also see 1.11). Sixty percent of respondents suggested one of the key benefits of early screening and diagnosis was that their relative might qualify for drug treatment and community service access. Similar views were reported by de Vries et al. (2012), in that a diagnosis of dementia is the path to service and support in the UK.

In NZ up to $60 \%$ of PWD do not engage with service and support services due to diagnosis being made late in the course of the disease (Brickell, 2012). Also, Māori continue to be under-represented in service utilisation, including dementia services, even though they have poorer health and shorter life expectancy than European New Zealanders. The cost of access to primary care is identified as one of the main barriers (Ministry of Health, 2013a). Connell et al. (2009) found similar access barriers in the USA and highlights the potential barrier of professional cultural insensitivity, resulting in black 
families being less likely to seek help and to be more likely to be diagnosed later in the dementia disease process and have a less smooth journey than other service users. Conversely, Connell et al. (2009) found that early diagnosis helped some family members and caregivers to be more understanding and patient with their ill family member and enabled them to make more-informed decisions on their behalf.

Another benefit for diagnosis disclosure that is emerging in the literature is the opportunity for family/whānau and significant others to 'prepare to care', a role that attracts descriptors such as 'near-to-super human' (Kitwood, 1997, p. 4), courageous, tender and generous (Alzheimers New Zealand, 2012). It is recognised that caregivers efforts can extend the independence of people with dementia and delay admission into the residential care setting, especially when the caregiver has access to multi-component interventions (Alzheimers New Zealand Incorporated, 2008; Boustani, Peterson, Hanson, Harris, \& Lohr, 2003b). These findings were upheld in the literature by Banerjee et al. (2006) who found significant decrease in permanent placements by $28 \%$ following a brief programme of caregiver support and counselling. Forbat (2003), argues that maintaining the polarity between the person with dementia and family members and/or caregivers, fails to explore the more complex relationships. de Vries et al. (2010) concurred with this finding and advised there was an urgent need to integrate perspectives of PWD and their family and caregivers to both improve understanding, and to address the complexities of care planning and care interventions. This view is supported by Spijker et al. (2008), who propose that engaging family caregivers in making choices about treatments and interventions distinguishes effective from ineffective support programmes. The above argument reiterates the crucial role of significant others, including family members and caregivers.

\subsection{Dementia subtypes}

The five most common dementia subtypes are: Alzheimers disease (AD), vascular dementia ( $\mathrm{VaD}$ ), mixed dementia $(\mathrm{AD}$ and $\mathrm{VaD})$, dementia with Lewy bodies, and frontotemporal dementia. In 1906 Dr Alois Alzheimer first described the pathological abnormalities in the autopsied brain of amyloid-beta peptide plaques outside the neurons (brain cells) and neurofibrillary tangles of tau protein within the neurons, the latter being parts of the dying nerve tissue, which are recognised as both the cause and hallmarks of 
AD (Prince et al., 2014). AD is the most common dementia subtype accounting for approximately $60-70 \%$ of cases, usually affecting older people with an average age of 78 years, although younger people are not immune (Perkins, 2013; Stephan \& Brayne, 2014). Women are more predisposed to AD than men (Alzheimers New Zealand Incorporated, 2008; Santibáñez, Bolumar, \& García, 2007). AD is slowly progressive and presents with decline of memory and cognitive ability, accompanied by loss of skills. Early symptoms include difficulty remembering names and recent events. Later symptoms include impaired judgment, confusion, disorientation, and behaviour changes (Alzheimer's Association, 2013c; Brickell, 2012; Perkins, 2013). The average lifespan, once symptoms have become noticeable to others, ranges from eight to seventeen years (Banerjee \& Wittenberg, 2009; Perkins, 2013).

$\mathrm{VaD}$, previously known as multi-infarct dementia or post-stroke dementia, is the second most common subtype, accounting for 10-20\% of all cases. It occurs due to problems with blood circulation to the brain and is related to ischemic, anoxic, or hypoxic brain damage and hypertension, diabetes and heart disease (Stephan \& Brayne, 2014; Prince et al., 2014). VaD has a more varied presentation than $\mathrm{AD}$, depending on where the vascular damage has occurred. For example, if the parietal lobe is damaged the person may have problems with numbers or recognising objects; if the frontal lobe is damaged the person may have problems making decisions (Perkins, 2013). However, the general presentation is one of poor concentration and communication, impaired judgment or impaired ability to plan steps needed to complete a task (executive function) (Stephan \& Brayne, 2014), as opposed to the memory loss often associated with AD. Physical symptoms, such as weakness of the limbs, paralysis or balance problems, may also be present along with slurred speech and diminished swallowing reflex (Stephan \& Brayne, 2014; Ekberg, Hamdy, Woisard, Wuttge-Hannig \& Ortega, 2002; Perkins, 2013). VaD tends to start at a younger age than $\mathrm{AD}$ and men are more predisposed to $\mathrm{VaD}$ than women (Perkins, 2013). Furthermore, Māori and Pacific peoples may be at more risk due to being prone to underlying conditions that damage blood vessels (Perkins, 2013). The average lifespan, once symptoms have become noticeable is highly variable (Stephan \& Brayne, 2014) from smoothly progressive to sudden decline (Alzheimer's Association, 2013b; Perkins, 2013). Mixed dementia, a combination of $\mathrm{AD}$ and $\mathrm{VaD}$, including mixed symptomatic presentation, is the third most common dementia subtype accounting for 10$20 \%$ of all cases (Alzheimer's Association, 2013b; Perkins, 2013). 
Dementia with Lewy bodies is the fourth most common subtype, accounting for $10 \%$ of all cases (Alzheimer's Association, 2013b). It presents with similar early stage AD symptoms; in addition, sleep disturbance, hallucinations and some muscle rigidity is likely (Stephan \& Brayne, 2014; Perkins, 2013). Lewy body dementia is closely related to Parkinson's disease as some of the symptoms, including muscle rigidity, are similar, and some people with Parkinson's disease develop dementia (Perkins, 2013).

The fifth most common sub-group is Frontotemporal dementia, which is a group of disorders rather than a single disease and accounts for 5-10\% of all cases (Alzheimer's Association, 2013b; Perkins, 2013). Early symptoms include changes in the person's personality and behaviour; however, there is no primary dysfunction in memory (Stephan \& Brayne, 2014). In contrast to $\mathrm{AD}$ and other dementias, behaviour changes are the primary presentations: being uninhibited, taking risks, neglecting personal hygiene, dressing oddly and having changes in emotion, speech and language, which occur despite other cognitive functions remaining initially unchanged (Alzheimer's Association, 2013c; Perkins, 2013).

\subsection{Dementia presentations}

Generally speaking, for all dementia types, there is a range of cognitive and noncognitive symptomatic presentations. Cognitive symptoms include decline in cognitive abilities: memory storage and retrieval functions, concentration, reasoning, decision making, and increased episodes of confusion. These symptoms affect a PWD's ability to function in daily life. Functional disabilities, which are serious enough to interfere with the most basic Activities of Daily Living (ADLs) include eating, drinking, toileting, dressing, bathing or showering, mobilising (Stephan \& Brayne, 2014; Perkins, 2013), all of which require coordinated movements of the arms, hands and digits (Cohen-Mansfield et al., 2006; de Bruin, Sacrey, Brown, Doan, \& Whishaw, 2008; Stineman et al., 2014; Verheij et al., 2012). Also, due to cognitive decline and preceding the loss of functional independence in completing ADLs, the more complex Instrumental Activities of Daily Living (IADLs) are being, or have been, eroded. This includes the ability to independently operate the telephone, manage money, prepare meals, use the oven, store and manage food in fridge and freezer, undertake light and heavy housework, shop for personal items, 
undertake self-transportation, and take medications (Pérès et al., 2008; Stineman et al., 2014). Loss of some of these abilities may be noticeable for up to 10 years prior to a diagnosis of dementia being made (Pérès et al., 2008).

Functioning is further impaired as a result of non-cognitive BPSD $^{5}$ such as disturbed perception, thought content, mood and behaviour (depression, anxiety, agitation, restlessness), apathy and sleep disturbance, incontinence, pacing/wandering, and repeating themselves (Bpac ${ }^{\mathrm{nz}}, 2013$; Perkins, 2013; Prince et al., 2014; Snowden, Sato, \& RoyByrne, 2003). The literature reveals that PWD usually present in primary care with declining memory and other cognitive symptoms, which tend to become the focus of care and treatment for up to four to seven years pre-diagnosis (Bamford et al., 2007; Banerjee \& Wittenberg, 2009; Campbell et al., 2008; de Vries et al., 2012; Iliffe, \& Manthorpe, 2004; Iliffe et al., 2003, 2006). Conversely, it is the non-cognitive emotional, behavioural and perceptual changes that families and caregivers find the most difficult to deal with (Alzheimer's Association, 2013b; Bpac ${ }^{\mathrm{nz}}$, 2013; Campbell et al., 2008; Kitwood, 1990, 1997; Perkins, 201 Small et al., 1997), and can result in institutionalisation (Bpac ${ }^{\mathrm{nz}}, 2013$; Mittelman, Roth, Haley, \& Zarit, 2004).

This dichotomous challenge of symptomatic presentations is being addressed through research and contemporary theory and practice that ensures inclusivity through engagement, communication, training and support for family members and caregivers who are acknowledged as a vital part of the care planning and provision solution for meeting the needs of, and improving the QOL for PWD, family members and caregivers (de Vries et al., 2010; Oyebode \& Parveen, 2016; Røsvik, Brooker, Mjorud, \& Kirkevold, 2013; Røsvik, Kirkevold, Engedal, Brooker, \& Kirkevold, 2011; Spijker et al., 2008). Additionally, through inclusive practices QOL can be improved by extending functional independence of PWD (Banerjee et al., 2006; Boustani et al., 2003b) and family and caregiver tolerance (Banerjee \& Wittenberg, 2009; Campbell et al., 2008; de Vries et al., 2010; Forbat, 2003; Mittelman et al., 2004; Spijker et al., 2008). Pharmacological

\footnotetext{
${ }^{5}$ There is no derogatory intent in the use of the term BPSD in this thesis. It is acknowledged that the term BPSD, is incongruent with person-centred care (Kitwood, 1990, 1997), embodied selfhood theories, (Kontos, 2003, 2004), and bio-psycho-social approaches to dementia (Cohen-Mansfield, 2014; Sabat, 2014). However, the term is commonplace in clinical practise, and in the grey literature that informs clinical practise interventions (Alzheimer's Association, 2015a; Alzheimer's Association, 2015b; Bpacnz, 2008, 2010, 2013; NICE-SCIE, 2006/2015); it was therefore considered to be an appropriate term for categorising a multitude of 'unmet need behaviours' for the purpose of this study.
} 
interventions are the most common approaches taken to address many behavioural and psychological symptoms of dementia.

\subsection{Pharmacological treatments}

There are relatively few pharmacological interventions currently licenced for the treatment of $\mathrm{AD}$ and even less choice for the treatment of other dementia subtypes (NICESCIE, 2006/2015). The two types of pharmacological treatments currently licenced for treating cognitive symptoms of $\mathrm{AD}$ are acetylcholinesterase (AChE) inhibitors and memantine, a partial antagonist of N-methyl-D-aspartate receptors (Birks, 2006; McShane et al., 2006; Rodda \& Carter, 2012). Acetylcholine is an important neurotransmitter that helps to send messages between neurons in the brain. Cholinesterase enzymes break down acetylcholine in the brain, resulting in falling levels of acetylcholine, which are associated with progressive loss of neurons and worsening symptoms (Birks, 2006; Rodda \& Carter, 2012). AChE inhibitors, donepezil, rivastigmine, and galantamine are considered first line pharmacological treatment for mild to moderate AD (Birks, 2006; Bpac $^{\mathrm{NZ}}$, 2010; NICESCIE, 2006/2015). They work by preventing or inhibiting the breakdown of the neurotransmitter acetylcholine (Rodda \& Carter, 2012). AChE inhibitors are not currently considered suitable for non-Alzheimer dementias or mild cognitive impairment, except as part of clinical trials (NICE-SCIE, 2006/2015; Rodda \& Carter, 2012). However, recent studies provide some evidence that $\mathrm{AChE}$ inhibitors are useful in other types of dementia, including dementia with Lewy bodies, Parkinson's disease dementia (Rodda \& Carter, 2012), and mixed dementias, where AD is a component (NICE-SCIE, 2006/2015; Rodda $\&$ Carter, 2012). There is no evidence for use in frontotemporal dementia and mild cognitive impairment (NICE-SCIE, 2006/2015; Rodda \& Carter, 2012).

Hansen et al. (2008) conducted a systematic review and meta-analysis of trials using AChE inhibitors (donepezil, rivastigmine and galantamine). The review revealed 14 of the 22 studies established that all three cholinesterase inhibitors showed a modest mean benefit of about 2.7 points on the Alzheimer's disease Assessment Scale Cognition Section of the 70-point scale, when compared to placebo, after three to six months of treatment. Most studies reporting measures of function such as ADLs also significantly favoured active treatment. There is no evidence however, that AChE inhibitors modify the neurodegenerative process, they simply provide symptomatic relief by slowing 
cognitive decline and loss of functional independence (Brickell, 2012; Hansen et al., 2008).

Glutamate is another important chemical neurotransmitter necessary for learning and memory that helps to send messages between the neurons. However, when neurons are damaged, glutamate is released in excessive amounts and becomes neurotoxic, causing damage to neurons and worsening symptoms (McShane et al, 2006). Memantine is an alternative pharmacological treatment that works differently to AChE inhibitors. It is a partial antagonist of N-methyl-D-aspartate receptors, so helps to "prevent excitatory amino acid neurotoxicity" without interfering with the physiological actions of normal physiological levels of glutamate required for normal brain functioning (McShane et al., 2006, p. 3). There is evidence that memantine provides some symptomatic relief by slowing down cognitive decline and loss of independence (Brickell, 2012; McShane et al., 2006). However, like AChE inhibitors there is no evidence that memantine modifies the neurodegenerative process.

The above licenced pharmacological treatments have produced only modest improvement in cognition and functioning and clinical research efforts continue to find more effective treatments for $\mathrm{AD}$ and other dementias, including efforts to better understand the role of sigma 1 receptors in regulating neural plasticity and growth, including advancements in the development of a multi-target drug involving sigma 1 receptors called ANAVEX2-73 (Koshibu, 2016). Multifactorial pathology such as AD may require combinational or multi-target therapy rather than monotherapy that targets a single step of the "vicious cycle of degeneration" in AD and other dementias (Koshibu, 2016, p. 80). Furthermore, Koshibu concludes that monotherapy may be responsible for the "disappointments in previous clinical trials" (p. 80). However, preliminary results of the ongoing ANAVEX2-73 phase 2a clinical trial report a "38\% improvement in the neurophysiological effect in 10 out of 12 patients...mostly on donepezil", with mild to moderate dementia (p. 80). The preliminary findings were announced in an Australian regional newspaper (Melbourne Herald Sun):

Alzheimer's disease breakthrough: Melbourne drug trial achieves amazing results....Patients have been able to regain abilities they had previously lost to the...horror disease. In one case, a patient regained the ability to play the piano. 
[Another] rediscovered her ability to paint. [Associate] Professor Macfarlane said, 'they seem significantly improved in terms of what they can do.... To me, seeing the real improvement to patients is much more important than seeing a statistically significant improvement on a rating scale.' (McArthur, 2016, p. 1)

In a population based study in Taiwan the average duration for acetylcholinesterase inhibitor administration was around 14 months, as it was usually terminated if the MiniMental State Examination score (MMSE) dropped by two points or the clinical dementia rating score worsened by one or more grades in a six-month period (Sun, Lai, Lu, \& Chen, 2008). In NZ, clinical recommendation is to review regularly and stop treatment if it appears ineffective or is not tolerated, similar to the UK (NICE-SCIE, 2006/2015).

Other pharmacological treatments widely used in dementia care practice include medications to treat non-cognitive or behavioural symptoms of dementia, including antipsychotics, anti-anxiety and hypnotic treatments (Sabat, 2014). Depression is the most common mental illness in adults and is associated with lower levels of QOL and high levels of disability in PWD (Prince et al., 2014; Snowden et al., 2003). Moreover, it is the emotional, behavioural and perceptual changes that families and caregivers find the most difficult to deal with, especially depression, agitation, wandering and incontinence, which can lead to institutionalisation (Alzheimer's Association, 2013b; Bpac ${ }^{\mathrm{nz}}$, 2013; Campbell et al., 2008; Kitwood, 1997; Mittelman et al., 2004; Perkins, 2013). Likewise, for PWD, depression and BPSD are two of the most significant mental health challenges faced when living in institutions such as nursing homes (Snowden et al., 2003), as is the potential for inappropriate medication use, such as polypharmacy of five or more drugs (Lau et al., 2010; Lai et al., 2012). Pharmacological treatments are only part of the answer. Campbell et al. (2008, p. 1082) discuss the need to consider "carer care" and not to solely focus on reducing the "objective load" of caring through the stabilising of cognitive and noncognitive BPSD, but also to address the "subjective interpretation by the carer" that has been shown to directly determine the burden of care.

\subsection{Global disease care burden and economic impact}

Global burden of disease measures the disease burden effect on both quality and quantity of life. Recent changes in the tools used to measure the burden of disease has 
seen dementia fall from $5^{\text {th }}$ to $9^{\text {th }}$ most burdensome condition for people over 60 years of age. However, Prince et al. (2015) are critical of how the global burden of disease for dementia is measured, reporting that the new measurements fail to take into account the full impact of chronic diseases where most of the burden comes from disability, care needs and societal costs rather than associated mortality, making the current measure unreliable for dementia. Recent studies show that health and social costs of dementia care in the UK almost match the combined costs of cancer, heart disease and stroke and exceed the combined health and social care costs of depression, stroke, alcohol abuse and osteoporosis in Sweden, and the costs of depression, hypertension, diabetes, ischaemic heart disease and stroke in Latin America and China (Prince et al., 2015).

The global economic impact of dementia in 2015 was US \$818 billion (Prince et al., 2015). In NZ, the economic impact of dementia in 2008 was estimated to be NZ $\$ 712.9$ million. By 2011, the cost was estimated to be $\mathrm{NZ} \$ 954.8$ million, of which $\$ 596.3$ million was for health system expenditure and $\$ 358.5$ million was for other financial costs (Alzheimer's New Zealand, 2012), an increase of NZ \$241.9 million in three years. An estimated increase of NZ \$80.63 million per year, if extrapolated, suggests that in 2017 the estimated economic impact of dementia in NZ could be near NZ $\$ 1.439$ billion. It should be noted that the figures above exclude disability costs, informal care costs and wider societal costs (Alzheimer's New Zealand, 2012; Prince et al., 2015).

Societal changes will also be evident in terms of the burden of caring for a family member with dementia. Currently, the burden is carried by women (de Vries et al., 2016; Kerse, 2014a; Kirkman, 2011; Ward-Griffin, Oudshoorn, Clark, \& Bol, 2007). An ongoing longitudinal study conducted by the University of Auckland, of 421 Māori and 516 non-Māori people, found that daughters were the most helpful with ADLs for Māori women and men and non-Māori women who lived alone or lived with others. However, daughters are not the most helpful to non-Māori men living alone or with non-related others; this role is provided by non-related others if they lived alone and spouses (wives) when they lived with others (Kerse, 2014b). However, a recent study found that approximately $40 \%$ of dementia caregivers in Canada and $41 \%$ in the USA are men, the majority being husbands (Robinson, Bottorff, Pesut, Oliffe, \& Tomlinson, 2014). It is expected that current demographic trends will result in more sons taking on this care role in the future (Grigorovich et al., 2016; Robinson et al., 2014). I was unable to find this 
level of data in NZ due to the lack of epidemiological studies in dementia, therefore most projections for $\mathrm{NZ}$ are estimates based on and corroborated by date-appropriate World Alzheimer's Report(s) (Alzheimers New Zealand, 2012), although early demographic and care burden data are being reported from LILACS NZ (Hayman et al., 2012).

The huge epidemiological, political, and social impact of dementia is evidenced by the literature. In response, determinations to stimulate action are being made by government departments and other strategic bodies, through strategic and policy documents, that focus on people with dementia as being a priority for investment for service and research, both in NZ and globally (Alzheimers New Zealand, 2010, 2012; Ministry of Health, 2013b; NICE-SCIE, 2006/2015; Prince, Prina, \& Guerchet, 2013; The Lancet Neurology, 2014). Priorities included research into the risks associated with the development of dementia.

\subsection{Risk factors: non-modifiable and modifiable}

There are several risk factors for developing dementia, including the nonmodifiable risk factors of: age, genes, family history, and Down syndrome (Prince et al., 2014; Santibáñez et al., 2007; van den Berg, \& Splaine, 2012). Research evidence appears to be weighted towards non-modifiable risk factors. Age is the best-studied risk factor for developing dementia, affecting $1 \%$ of people 65 years and older and up to $25 \%$ of people 85 years and older (Alzheimers New Zealand Incorporated, 2008; van den Berg, \& Splaine, 2012). Having a first-degree relative with a history of AD and/or having the apolipoprotein E e4 genotype increases the risks of developing AD (Boustani et al., 2003b; van den Berg, \& Splaine, 2012). Whereas AD and VaD share the same lifestyle and cardio-vascular risk factors (van den Berg, \& Splaine, 2012), there are strong familial indicators for frontotemporal dementia (Alzheimers New Zealand Incorporated, 2008; van den Berg, \& Splaine, 2012). There are also gender differences. By the age of 80 women have a slightly higher risk, rising to $13 \%$ higher at the age of 94 than men of developing AD due to women living longer (Alzheimers New Zealand Incorporated, 2008; Santibáñez et al., 2007). Conversely, men are more susceptible to developing VaD because they are more prone to developing stroke or heart disease than women (Perkins, 2013). Although non-modifiable risk factors cannot be modified, they could be used to identify those most at risk of developing dementia for early screening and intervention programmes (Prince et 
al., 2014). Iliffe and Orrell (2006) advocate for identifying and addressing the unmet health needs of older people, rather than comprehensive screening.

Prince et al. (2014) and van den Berg and Splaine (2012) advocate a more positive view of the future through self-determination, the rewards of which are risk reduction, by focusing on the modifiable risk factors that have the potential to reduce risks associated with developing dementia in later life through appropriate lifestyle and cardiovascular risk management. Prince et al. (2014) conducted a meta-analysis of systematic reviews relating to protective and modifiable risk factors and present their findings in four useful domains of concern: development and early-life, psychological, lifestyle and cardiovascular risk, which are summarised below.

Development and early life events determine final brain size. A larger brain volume has increased neurons and or synapse, termed "brain reserve" (Prince et al., 2014, p. 14), which may be related to delayed onset of clinical symptoms of dementia when brain pathology occurs. Risk factors include intrauterine environment, birth weight, the number of siblings, birth order and growth, and development factors such as nutrition. Also, socioeconomic factors such as enriched environments and educational opportunities in the first years of life may have a beneficial effect. In addition, higher number of years of formal education and higher occupational attainment are potential protective factors against the risk of developing dementia and are explained by the term "cognitive reserve" (p. 17), a term relating to a greater reserve of brain functioning in terms of cognitive processes where more complex and efficient neural networks compensate when dementia related brain pathology occurs. Conversely, early negative events may affect the brain structure and development due to stress reactions, which affect neuro-endocrine processes.

The psychological domain embraces the risks associated with depression, psychological distress, and psychological sleep disturbance. Depression is the most common mental illness in adults and is associated with lower levels of QOL and high levels of disability (Prince et al., 2014). The incidence and prevalence can be reduced through appropriate pharmacological and non-pharmacological treatments. Although the nature of association between depression and other cognitive disorders, including dementia, is evident in the literature, the association is not fully understood. What is clear is that dementia and depression often co-exist and depression may increase the risk of 
developing dementia. Psychological distress is often associated with depression and is a term used for "the state of feeling pressure of stress" (p. 30). It is very common in all populations and can be modified and improved with lifestyle, environmental, pharmacological, and non-pharmacological interventions. However, the evidence remains insufficient to advocate preventative interventions in the general population. Finally, psychological sleep disorders are a collection of conditions that are symptomatic of circadian rhythm alteration. They are "strongly related to behavioural changes... [and] are exacerbated in people with dementia" (p. 35). However, it is currently unclear if the changes in sleep are cause or effect of the underlying brain pathology of dementia. Pharmacological interventions, such as sleeping pills (benzodiazepines), have been associated with an increased risk of developing dementia in people with minimal exposure, but not with high exposure (Gray et al. 2016), therefore other non-pharmacological psychosocial interventions may be alternative pathways to reduce the risk or delay the onset of dementia associated with psychological sleep disturbance.

Modifiable lifestyle factors include smoking, alcohol, physical activity, diet, and cognitive stimulation. Findings of Prince et al. (2014) indicate a possible positive risk association between smoking and the onset of dementia in later life. Smoking has been found to exacerbate $\mathrm{AD}$ and $\mathrm{VaD}$ pathology due to underlying vascular, neurovascular and neurodegenerative pathology. However, other studies found smoking reduced neurodegenerative pathology, potentially protecting against some neurodegenerative conditions, such as Parkinson's disease and Lewy body dementia. In addition, some substances in cigarette smoke, for example nicotine, may offer protection of the brain from "ischaemic and excitotoxic cell damage" (Author, p.43). Like smoking, there are pros and cons for alcohol consumption, which in moderate amounts has been found to have neuroprotective functions. Conversely, heavy or excessive alcohol has been found to produces cerebral volume loss of white matter related to memory processes and visuospatial functioning.

Regarding the association of physical activity and risk of developing dementia the evidence is inconsistent. Observational studies in this area suggest that physical activity may be associated with up to a $40 \%$ reduced risk of developing dementia due to the associated benefits of control of other comorbid modifiable cardiovascular risk factors such as hypertension, obesity, dyslipidaemia and diabetes (Prince et al., 2014). Similar 
beneficial effects are associated with eating a healthy diet. Cross-sectional studies indicate that healthy older people tend to have a healthier diet, richer in fruit and vegetables, rather than process carbohydrates, meat and fats; therefore, as with physical activity, healthier diets have associated benefits of control of other comorbid modifiable cardiovascular risk factors. Finally, a large number of observational studies looking at cognitive stimulation interventions have revealed consistent results relating to cognitive activity in late-life may be beneficial for brain structure and function. Cognitive stimulation is expanded in section 2.2.3).

In addition to the four domains of modifiable risk proposed by Prince et al. (2014), Alzheimers New Zealand Incorporated (2008) highlight a fifth domain relating to occupational exposure to pesticides, solvents, high magnetic fields, lead and aluminium, which may increase the risk of developing AD. Santibáñez et al. (2007) challenge the quality and consistency of the research findings into occupational risk exposure other than exposure relating to pesticides, in which studies were of greater quality and reported statistically significant association. Associations were less consistent for solvents and electromagnetic fields and absent for lead and aluminium. In addition to understanding dementia from an epidemiological perspective, a philosophical viewpoint of dementia in relation to thought and thinking, mind and body is useful in order to understand the disease process.

\subsection{Philosophical positioning of thought, thinking, mind and body}

Observed through the lens of Cartesian philosophy, people with $\mathrm{AD}$ or other dementias lose their ability to think, due to the destruction of the neurons in the brain. The person (as a mind) is progressively destroyed and begins to detach from the corporeal matter (physical body) as their need for interaction diminishes to the point at which no person remains. The epistemology of René Descartes (1596-1650) is "I am thinking, therefore I exist (je pense donc, je suis, or cogito ergo sum" (Descartes, 1997, p. XIV). Ontologically Descartes concludes that the world is made up of three existent substances: god, thought and matter (Descartes, 1993, 1997), all of which lie on a continuum of existence and interconnectedness. A continuum where the substance of god exists as a supremely, omniscient perfect being. The substance of thought and thinking exist as the essence of the mind, and the substance of all other material that constitutes all other things, 
including the human body, fall into the category of matter (Brown, 2001; Descartes, 1993; Hatfield, 2014). Proof that the body exists is a Cartesian doubt, as existence of the body is known only through interconnectedness of the senses (Descartes 1993, 1997). When Descartes (1993) refers to the corporeal body, he describes his understanding as emerging through "the light of nature" (p. 54), or imagination, rather than through object reality as determined through thought and thinking. It is through non-omniscient and therefore potentially deceitful nature that Descartes attributes some bodily functions and behaviours, which he refers to as intuit or instinctive associative memory and mechanistic actions, and describes them as responses to sensing the world around us; a response that does not involve consciousness or intellect. Separating the thinking mind from the corporeal body as proposed by Descartes, is often referred to as mind-body dualism, where each person is a mind or thinking substance (thought), which is intermingled, interconnected, and interactive with a corporeal physical body, a material substance (matter), which is only known to exist through extension of the senses (Descartes, 1993, Brown, 2001).

This Cartesian perspective is reflected in the literature in which PWD are described as having lost or separated from their self, self-esteem, or self-identify (Aminzadeh, Byszewski, Molnar \& Eisner, 2007; Cohen \& Eisdorfer, 1986; Cohen, Kennedy, \& Eisdorfer, 1984; Millett, 2011). Other writers go further and evoke an image of a body without a mind by describing PWD as diminishing or delayering until nothing remains of self (Davis, 2004; Fontana \& Smith, 1989), or what remains of self lies in the realm of unbeing a self (Kitwood, 1997), or a non-person (Millett, 2011), all of which allude to Cartesian mind-body dualism that is inextricably linked to the mind and not to both the mind and the body. This theoretical concept that the person (as a mind) is progressively destroyed and begins to detach from the corporeal matter (physical body) is challenged in section 2.4. It is a philosophical position that continues to be visible in contemporary dementia literature, however, it is a position that has the potential to stigmatise PWD and others who walk alongside them.

\subsection{Dementia and stigma (external and internal)}

Historically, dementia has suffered from limited awareness and understanding, combined with the stigma attached to both mental illness and old age. Goffman (1963) describes stigma as an identity discrepancy between stereotypical expectations of society 
in relation to a person's "virtual social identity... [and their] actual social identity" (p. 12), and an "attribute that is deeply discrediting" (p. 13). Kitwood (1990) referred to the stigmatisation of PWD as "malignant social psychology" (p. 181), a term he used to describe the undermining and stigmatising of PWD through disempowerment, labelling, invalidation, banishment and objectification. Nolan, McCarron, McCallion, and MurphyLawless (2006a) illuminate how PWD and their caregivers are subject to a "double edged sword...[of] living with dementia and the potentially negative attitudes and behaviours of others" (p.19). More recently, Swaffer (2014) critically reviewed the stigma and dementia literature, through the lens of a younger person living with early onset dementia, and found the language used about people with dementia by researchers, healthcare professionals, and associations advocating for, and supporting PWD, to be "dehumanizing" (p. 13).

The use of euphemisms, such as 'confusion' and 'short-term memory loss', and 'memory problems of old age' feature in conversations amongst people with dementia, their families and health professionals, especially in diagnosis disclosure meetings, due to professionals, and occasionally caregivers, trying to avoid causing more harm than good, or to avoid the upset of dementia disclosure (Bamford et al., 2004). However, Swaffer (2014) is critical of such narrative, which she argues continues the stigmatisation of PWD, and delays access to appropriate information, support, and care interventions. Moreover, Swaffer concludes that stigma and discrimination are compounded by low numbers of people with dementia taking part in research activities, or contributing to conversations and consultations.

Goffman (1963) describes people like Swaffer as sympathetic others, who either share the stigma or who become wise through association with the stigmatised individual as having the ability to provide "exemplary moral tales in biographical and autobiographical form illustrating a desirable code of conduct for the stigmatized" (p. 37). Greenwood, Gordon, Pavlou, and Bolton (2016) argue that volunteer befrienders have the potential to develop relationships with PWD that challenge the stigma surrounding dementia, however, the paradoxical processes inherent in such relationships needs to be explored further.

The stigma literature is multifaceted, depending on the sociological or psychological lens through which it is viewed (Link \& Phelan, 2001). When looking at 
the sociological effects of stigmatisation Nolan et al. (2006a) focus on what are considered to be external stigmatising events such as the experiences of PWD and their caregivers when navigating complex service and support systems. This often results in isolation and creates barriers to service information. Similar views are expressed by Link and Phelan (2001) and Reidpath, Chan, Gifford and Allotey (2005). Of relevance to this study, are the internal or psychological emotional consequences of stigmatisation, which impact negatively on the health and wellbeing of the person being stigmatised. The stigma of a dementia diagnosis causes complex emotional responses. Aminzadeh et al. (2007) theorise three broad categories of emotional response to dementia diagnosis disclosure, two negative and one more hopeful. Firstly, lack of insight and/or active denial, for example, not noticing symptoms or noticing and covering them up. Secondly, grief/crisis responses, associated with anticipated losses: autonomy, competency, self-esteem, sense of control, valued lifestyle, social roles and relationships, and the most feared loss of all loss of self-identity. In contrast, the third emotional response is one of self-determination through positive coping and adaptive responses: noticing, revealing, disclosing, and confirming that there is a problem and maximising retained strengths and adapting accordingly.

These categories are similar to those reported by Cohen et al. (1984), who expanded on the grief responses by incorporating sadness, anger and guilt, and Keady and Nolan (1995) who contributed the categories of decline and death. Emerging from the above literature is the theme that the whole trajectory of the disease process needs to be constantly reappraised, which was articulated by Pearce, Clare and Pistrang (2002) as a need to balance the desire of the person with dementia to maintain a prior sense of self, against the need to re-construct self through constant re-appraisal as the disease progresses. To facilitate reconstruction of self, an understanding of human memory and learning is required.

\subsection{Memory and learning}

Human memory is commonly described as a "Multi-Store Model" or "AtkinsonShiffrin Model" (Figure 1), having three storage areas (sensory, short-term, and long-term) through which information is received, processed, stored, and retrieved (Sutton, 2010). The language used to describe key elements of long-term memory is contested in the 
literature where it appears to be dependent on epistemological perspectives; for example, philosophers 'habit memory' is 'procedural memory' and 'propositional memory' is 'semantic memory"' in psychological theory (Sutton, 2010), see Figure 2.

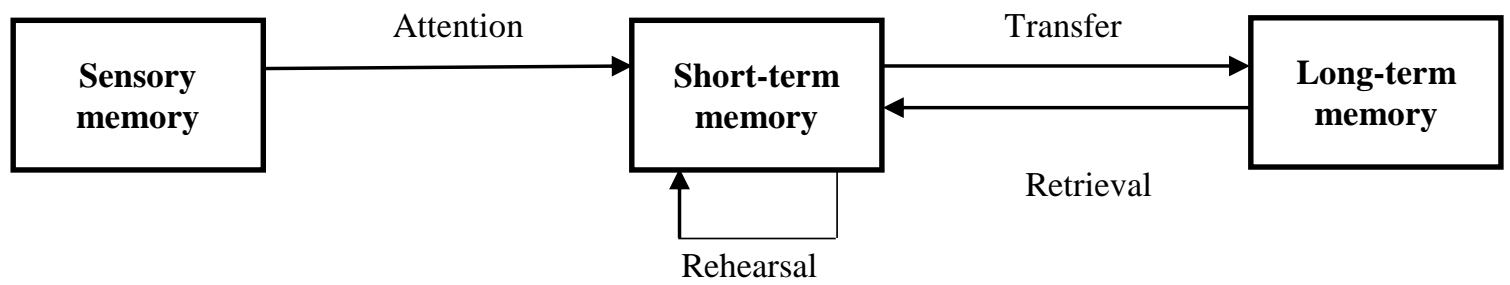

Figure 1. Multi-store model of memory

Informed by Atkinson-Shiffrin Model (1968). Image available through Creative Commons Attribution Share Alike. Downloaded from https://en.wikipedia.org/wiki/Atkinson\%E2\%80\%93 Shiffrin_memory

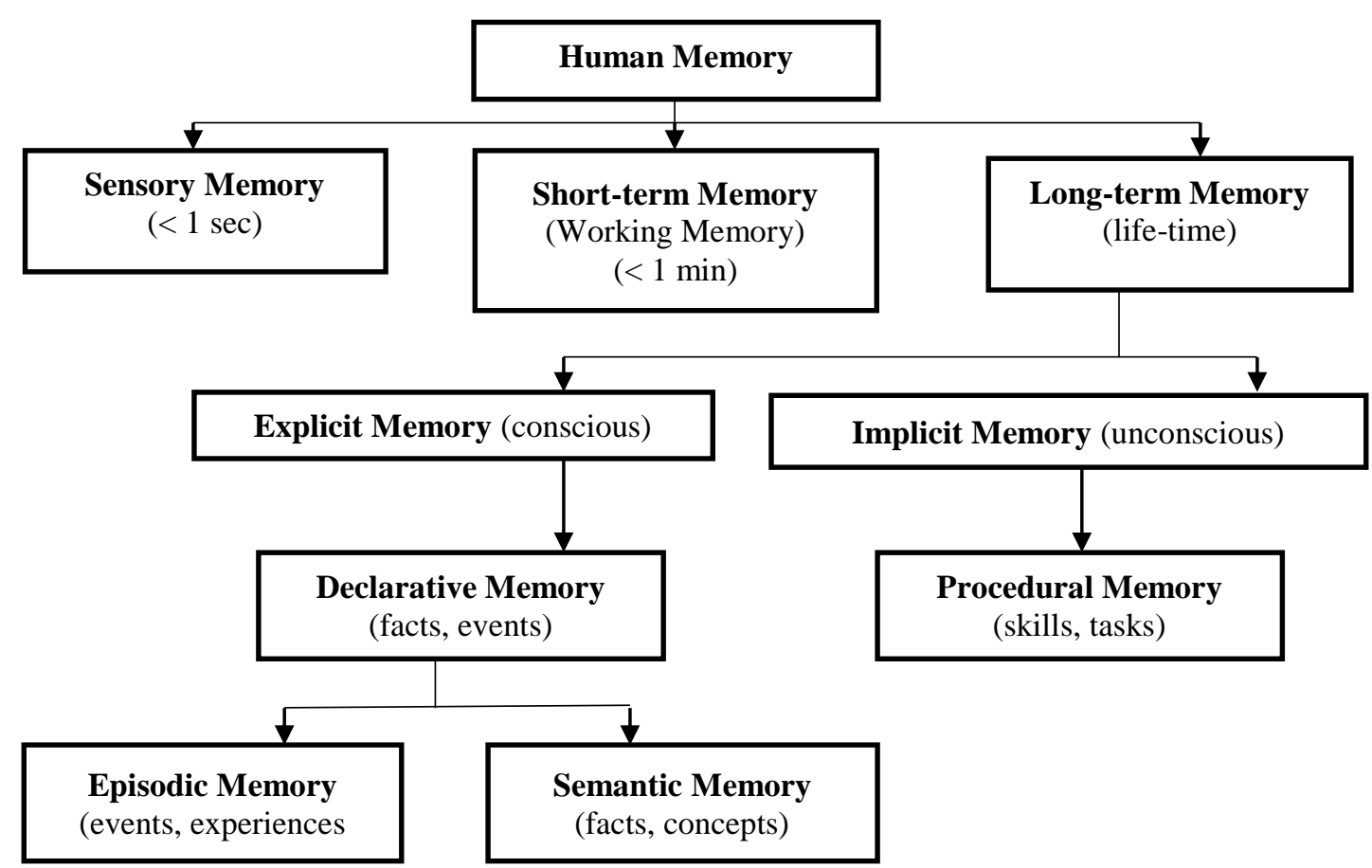

Figure 2. Types of human memory

Reformatted. Original by Luke Mastin. Permission to use image granted on 23/11/15 from http://www.humanmemory.net/types.html

Explicit memory refers to the conscious recollection of past events (Kihlstrom, Dorfman, \& Park, 2007). One of the functions of explicit memory is to remove errors during the cognitive learning process by drawing on past knowledge and experiences (Kihlstrom et al., 2007). Baddeley and Wilson (1994) reiterate the importance of this function in relation to the learning process and in the choice of techniques used to facilitate learning, such as techniques targeted at explicit or implicit memory pathways (see section 2.2.7). 
In $\mathrm{AD}$, marked deficits in explicit episodic memory ${ }^{6}$ is the earliest symptomatic presentation (Alzheimer's Association, 2013c; Clare et al., 2000; Evans, Levine \& Bateman., 2004). People with dementia lose the capacity to remove errors due to functional damage to the explicit memory pathway (Baddeley \& Wilson, 1994; Clare et al., 2000). Because of this loss, it is difficult for PWD to remember and/or correct their errors when trying to learn or re-learn (Baddeley \& Wilson, 1994; Clare et al., 2000; Evans et al., 2004; Jokel \& Anderson, 2012; Jokel, Rochon, \& Anderson, 2010); for example, calling someone by the wrong name and then remembering the wrong name, rather than the correct one (Evans et al., 2004). In dementia, errors made during learning are strengthened in implicit memory through the priming effect upon subsequent experience, thought, or action (Kihlstrom et al., 2007; Yamaguchi, Maki, \& Yamagami, 2010). Therefore, learning is more efficient if errors can be avoided during the learning process (Evans et al., 2004; Sidman, 2010; Terrace, 1963).

In contrast, implicit procedural memory and some elements of short-term working memory are relatively unchanged in the early and middle stages of AD (Alzheimer's Association, 2013c), therefore learning and re-learning by PWD should be possible if techniques not emphasising error are utilised. Figure 3 exemplifies the role of explicit and implicit memory in relation to learning a basic task.

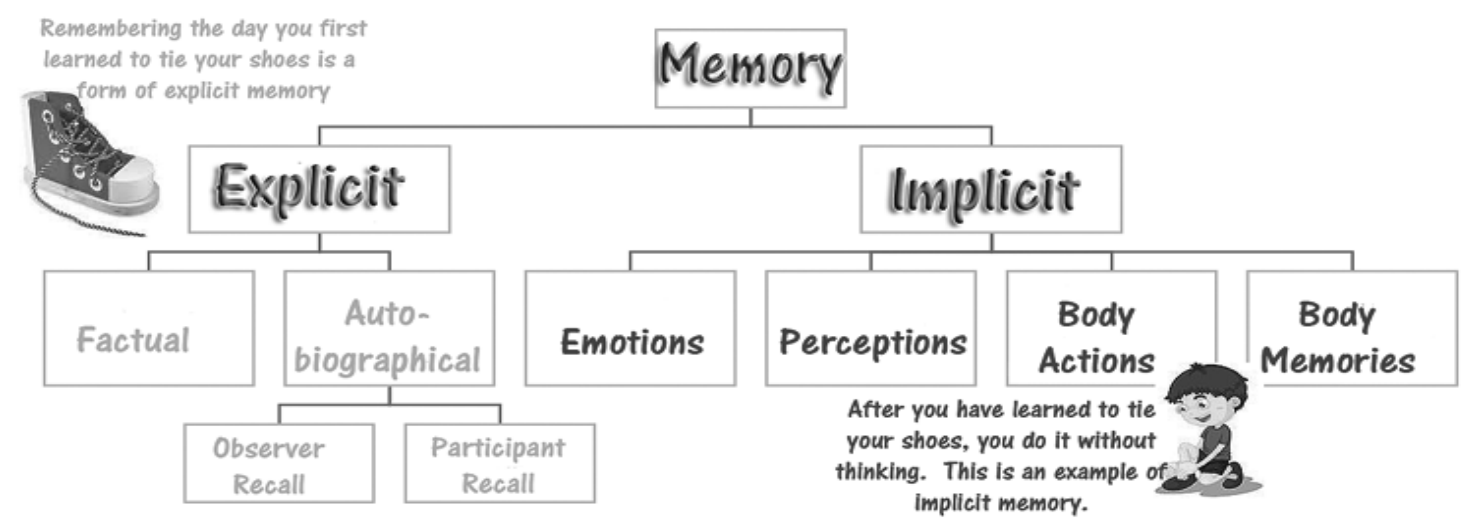

Figure 3. The role of explicit and implicit memory in relation to a basic task

Image available (reproduced) through Creative Commons Attribution Share Alike. Downloaded from http://www.dissociative-identity-disorder.net/wiki/Memory

${ }^{6}$ Episodic memory - anterograde (directed forward). 
The example demonstrates remembering what was learned and recalling the experience is a form of explicit (conscious) memory. However, once the task is learned, it can be completed without thinking; a form of implicit (unconscious) memory, and a natural body action that is stored as a bodily memory and draws on the implicit memory pathway. Tailby and Haslam (2003) could not clearly distinguish between the implicit or residual explicit theories, and recommend that both implicit and explicit memory processes may benefit from specific errorless learning (EL) interventions in PWD. What is clear is the importance of understanding how memory is affected in dementia and how best to work with PWD in an effort to sustain independent functioning, through relearning forgotten tasks and learning new things that meet the needs of the individual, both of which are important aspects of QOL.

\subsection{Quality of Life as conceptualised in this thesis}

There is insufficient data on the pattern of association between QOL and other important clinical outcome variables in $\mathrm{AD}$ and other dementias. Direct assessment of QOL in dementia research has been largely ignored due to logistical, practical and conceptual issues (Brod, Stewart, Sands, \& Walton, 1999; Oyebode \& Parveen, 2016; Selwood, Thorgimsen, \& Orrell, 2005). QOL is multi-dimensional and often reported subjectively (Hale et al., 2012; Woods et al., 2012). It is considered to be a complex phenomenon to measure (Banerjee et al., 2006; Edvardsson \& Innes, 2010; Woods et al., 2012), encompassing social, psychological and physical domains (Bhattacharya, Vogel, Hansen, Waldorff, \& Waldemar, 2010; Missotten et al., 2008). Studies that rely on discrete measures of cognition and functioning to draw assumptions that when significant findings are observed in these domains that overall QOL must be improved are challenged (Banerjee et al., 2006; Woods et al., 2012). Other studies refer to the subjective selfreporting of QOL and suggest additional objective research efforts (Edvardsson \& Innes, 2010; Woods et al., 2012). The philosophical, ontological, and epistemological underpinnings of this current study were designed to facilitate the integration of a broad range of both objective and subjective aspects of QOL. The dimensions of QOL within this thesis are bounded by the maintenance of cognition, the absence of depression and anxiety, the global measure of QOL in AD and evidence of improved functioning. 


\subsection{Thesis overview}

This thesis is presented in six chapters. This first chapter presented an introduction to the thesis, including my research interests and justification for undertaking this study. The background literature positions and contextualises this thesis, providing an overview of dementia demographics and prevalence. I then provide a definition of dementia and critically explore dementia diagnosis, symptomatic presentation, and current pharmacological treatments. Following which the global disease care burden, economic impact, and risk factors for developing dementia are critically explored. I then present a philosophical overview of the mind and body according to Descartes (1993; 1997). An alternative view of the mind and body is revealed through a glimpse at the stigma literature, and what is known about how the human memory works. The chapter concludes with a statement about how QOL is conceptualised within this thesis.

Chapter Two presents a critical review of the current evidence and identifies the gaps in knowledge relating to the provision of computer-assisted exergaming with Able$\mathrm{X}$, for PWD. The chapter provides a background into non-pharmacological, psychosocial interventions, which are often used with this population in an effort to improve cognition, and in so doing, mediate other improvements such as functioning and QOL. A description of cognitive stimulation is followed by a review of the brain training, gaming, and exergaming literature to expose the differences between these modalities, and to examine the ameliorative effects of computer-assisted exergaming. I then widen the focus to enable a comprehensive review of emerging areas of research into provision of cognitive oriented therapies for PWD, which includes the embryonic research relating to computer-assisted interventions for the provision of cognitive and functional rehabilitation, and the theoretical foundations of EL (Terrace, 1963). The review is extended beyond cognitive interventions to include theoretical perspectives and critical analysis of models of 'personcentred care', which has been shown to have positive or negative potentiality in relation to the outcomes of cognitive oriented interventions for PWD. The chapter concludes with a review of the epistemology of 'embodied selfhood' (Kontos, 2003, 2004), and how this epistemology enables us to understand and interpret the actions of PWD. The chapter concludes with a summary of key theoretical influences.

In Chapter Three, I critically examine the philosophical, ontological, epistemological, and methodological positioning of the study. I explore and justify my 
rationale for using social pragmatism, combined with interpretive epistemology, within a collective convergent parallel CS methodological approach. I then offer a detailed discussion of strengths and limitations of CS design. As CS is both a methodology and a method, I have chosen to present them within the same chapter for coherency. An in-depth description of the multiple methods I used to collect, manage and analyse my research data is presented, followed by a reflexive discussion on trustworthiness and rigour. Reflexivity is one of the aspects of rigour and I strive to make apparent throughout the chapter how my multiple positions influenced the study's methodological positioning and the research fieldwork. The chapter concludes with an examination of key ethical dilemmas relevant to researching vulnerable populations.

In Chapter Four, I present the first findings chapter, 10 case studies that describe and explain the computer-assisted exergaming experiences of each participant. The chapter opens with an explanation of the terms used to describe the proxy voices within each case and how each of the voices and the context in which these voices were documented can be recognised by the reader. The case studies are not uniformly presented in terms of demographic, personal, and clinical characteristics; this data is provided to the reader in the second findings chapter, Chapter Five. To complement the data generated through the process of CS methodology, I draw on a broad range of quantifiable outcome measures to validate my qualitative findings within each individual case.

Chapter Five is the second findings chapter. In this chapter, I critically explore the similarities and differences across the 10 cases studies described in Chapter Four. I begin with an overview of the demographic, personal, and clinical characteristics of each case and a synopsis of computer-assisted exergaming with Able-X in terms of exergaming engagement, frequency, and game description. Following this overview, I utilise a theoretical framework of 'embodied selfhood' (Kontos 2003, 2004) and the principle of EL (Terrace, 1963) to examine in detail five emergent themes and six associated novel concepts. The first three themes have a focus on the gaming environment: gaming initiation, engagement, and progress. Themes four and five explore the similarities and differences across each case in terms of the two main findings of improved hand-eye coordination and improved aspects of QOL. The chapter concludes with evidence of how these findings translated from the gaming room into the everyday lives of participants. 
Chapter Six concludes this thesis. The chapter begins with a critique of the methodology and methods, in which I reiterate my justification for utilising case study, and summarise the key strengths and limitations of this study. I make it clear how this research contributes to methodology and methods through the emergent, but comprehensive study design. I make explicit how this research contributes to knowledge, by providing new insights and understandings related to the provision of computer-assisted exergaming with Able-X and EL strategies. An overview of the implications for practice is followed by recommendations for future research and a summary of future publications, two of which are ongoing. The chapter closes with a concluding statement, which is followed by a poem that embraces the key theoretical concepts that bind this thesis, and contains glimpses of the findings that emerge from this research. The poem is dedicated to all my participants and sympathetic others, who share the journey and become wise through association. 


\section{CHAPTER TWO: LITERATURE REVIEW}

\subsection{Introduction}

One of the most common fears for PWD is loss of the 'mind' (Davis, 2004; Fontana \& Smith, 1989; Kitwood, 1997) or loss of 'self-identity' (Aminzadeh et al., 2007 Cohen \& Eisdorfer, 1986; Cohen et al., 1984; Millett, 2011) as the disease progresses. There is no cure for $\mathrm{AD}$ or other dementias, but there are many non-pharmacological interventions that have been shown, through robust research, to be as effective as pharmacological treatments in temporarily slowing down cognitive and functional decline and have been associated with improved QOL and wellbeing in this population. There has been rapid development of psychosocial interventions for PWD and supporting literature. Due to the word limitations of this thesis, this review is limited to the main findings of key critical and systematic reviews focusing on the main cognitive oriented psychosocial interventions, along with additional literature that is relevant to the argument laid out in this thesis, including literature relating to brain training, assistive technologies, and the role of computer-assisted exergames as a conduit for rehabilitation.

In this chapter, I begin by providing an overview of the non-pharmacological psychosocial interventions that are available to PWD. Following a critical description of cognitive stimulation and the four main types of cognitive oriented interventions, I then review the brain training, gaming, and exergaming literature to expose the differences between these modalities, and to examine the ameliorative effects of computer-assisted exergaming. Following this, I explore the emerging literature on computer-assisted interventions for people with neurological assault, including PWD. I then critically examine the foundations of learning without errors work of Terrace (1963), and explore how this cognitive rehabilitation strategy has evolved into EL rehabilitation of PWD. The penultimate part focuses on the theory of personhood and person-centred care, an important concept for people undertaking research with this vulnerable population. The chapter concludes with a critical review of 'embodied selfhood' (Kontos, 2003, 2004), in so doing, I address one of the most common fears of PWD, that of loss of 'self', by demonstrating that the body is agential at a pre-reflective level, and that no matter how ravished the brain is, 'selfhood' is never lost, it is 'embodied' and intentionally capable. 


\subsection{Non-pharmacological interventions}

Non-pharmacological psychosocial interventions are increasingly used independently, or alongside pharmacological interventions, both internationally and in NZ (Table 1). Evidence from a large number of observational studies indicate that cognitive activity programmes may be beneficial for both brain structure and function. However, the evidence is limited and caution is advocated as the world awaits the results of randomised control trials on cognitively healthy subjects and on people at greater risk of developing dementia, to establish if lower cognitive activity levels is a prodromal sign of the disease in those who go on to develop dementia (Prince et al., 2014).

Table 1. Psychosocial interventions

\begin{tabular}{|c|c|}
\hline $\begin{array}{l}\text { Psychotherapeutic } \\
\text { Interventions }\end{array}$ & $\begin{array}{l}\text { Behaviour-oriented } \\
\text { - Behavioural management training/Scheduled toileting } \\
\text { - Caregiver training } \\
\text { Emotion-oriented } \\
\text { - Reminiscence therapy } \\
\text { - Validation therapy } \\
\text { - Supportive psychotherapy } \\
\text { - Sensory integration/Simulated presence therapy } \\
\text { Cognition-oriented } \\
\text { - Cognitive stimulation/training } \\
\text { - Reality orientation } \\
\text { - Cognitive Stimulation programme/therapy } \\
\text { Stimulation-oriented } \\
\text { - Recreational therapy/Art therapy } \\
\text { - Multisensory stimulation/Aromatherapy/Massage } \\
\text { - Animal-assisted therapy } \\
\end{array}$ \\
\hline Social Interventions & $\begin{array}{ll}\text { - } & \text { Daytime/night-time supervision assessment } \\
\text { - } & \text { Occupational therapy functional and safety assessment } \\
\text { - } & \text { Living environment modifications } \\
\text { - } & \text { Abuse/neglect assessment } \\
\text { - } & \text { Driving assessment/Gun licence assessment } \\
\text { - } & \text { Home health agency/Home cleaning service } \\
\text { - } & \text { Meals on Wheels } \\
\text { - } & \text { Financial/estate planning } \\
\text { - } & \text { Health care power of attorney/advanced directives } \\
\text { - } & \text { Long-term care facility }\end{array}$ \\
\hline Family Interventions & $\begin{array}{ll}\text { - } & \text { Caregiver psychoeducation } \\
\text { - } & \text { Respite care } \\
\text { - } & \text { Support groups } \\
\end{array}$ \\
\hline $\begin{array}{l}\text { Spiritual, religious } \\
\text { and Cultural }\end{array}$ & - Biography \\
\hline
\end{tabular}

Adapted from Brickell, K. (2012). Research review educational series: Treatment of Alzheimer's disease in New Zealand. Research Review, and NICE-SCIE (2006/2015). Dementia: Supporting people with dementia and their carers in health and social care: Clinical guideline 42. London, England: Author. 
Meanwhile, there is a growing literature and body of evidence that some psychosocial and psychotherapeutic interventions may have similar outcomes as pharmacological interventions, including significant improvement in both cognition and QOL (Aguirre et al., 2010; Ballard et al., 2011; Oyebode \& Parveen, 2016; Requenta et al., 2004; Tarraga et al., 2006; Woods et al., 2012). Oyebode and Parveen (2016) propose that psychosocial interventions "harness the leeway that exists to promote well-being [in dementia], in the absence of a cure...and enable people with dementia to live well now" (p. 2). As can be seen in Table 1, there are a multitude of non-pharmacological interventions. This literature review focused on the interventions where the evidence is the most consistent, that is in the cognition-oriented interventions.

\subsubsection{Cognitive stimulation: The evidence base}

Cognitive stimulation is a global approach that targets both explicit and implicit memory pathways, and is concerned with both cognitive factors: attention, concentration, orientation, different types of memory, visual-constructive abilities, executive function, and verbal fluency and psychological factors: self-confidence, motivation, socialisation, and affective states (Cantegreil-Kallen, de Rotrou, \& Rigaud, 2009). Cognitive stimulation works by reinforcing the 'cognitive reserve' capacity of the brain, where more complex and efficient neural networks compensate when the brain ages, or when dementia and non-dementia brain pathology occurs (Ballard et al., 2011; Cantegreil-Kallen et al., 2009; Prince et al., 2014). The theory relating to the effects of cognitive stimulation is based on the notion of 'cerebral plasticity', which allows the brain to form new synaptic connections to take over functions of other parts of the brain that may be damaged (Hallett, 2001; King et al., 2010; Prince et al, 2014). Integral to this theory is "experiencedependent neural plasticity", which is based on the notion that environmental input can influence the generation of new brain connections (Gelfand, Vick \& Lewis, 2014, p. 55).

A systematic Cochrane review of the literature on cognitive stimulation was conducted by Woods et al. (2012). All RCTs, conducted between 1979 and 2011 for cognitive stimulation for dementia, which included a cognitive measure, were included $(n=15)$. A further 68 were excluded because they did not fit the inclusion criteria and two because they were ongoing. The authors were critical of some of the studies, which were of varying quality, small size, and limited in terms of details in respect to randomisation methods used. A meta-analysis involving 718 participants (407 receiving cognitive 
stimulation, 311 in control groups) was conducted. Primary analysis revealed that cognitive stimulation consistently benefitted cognitive functioning, and these benefits were maintained at one to three-month follow-up. Secondary analysis on a smaller sample revealed self-reported benefits in QOL and wellbeing and positive staff ratings for communication and socialisation, all of which were reported as being mediated by improved cognition. No self-reported or staff rated differences were found in relation to mood, behaviour or ADLs in people with dementia. Additional research into the significance of longer-term cognitive stimulation programmes was recommended, as was the need for more exploration of other outcomes, such as cognition mediated QOL. The authors conclude that cognitive stimulation programmes consistently provide cognitive benefits for people with mild to moderate dementia, over and above any medication effects, including the effects of pharmacological treatments for AD. Also, the authors recommend that all people with mild to moderate dementia should have access to cognitive stimulation interventions. This review is comprehensive and logically presented. The findings are convincing and widely generalisable. The conclusions and recommendations are consistent with the NICE-SCIE (2006/2015) guideline, and reinforced by Prince et al. (2011, 2014). However, the authors only focused on studies that described cognitive stimulation interventions targeting cognitive and social functioning, or studies that focused on reality orientation.

A second critical review conducted by Ballard et al. (2011) had a wider scope than Woods et al. (2012). The review specifically focused on cognitive and functional symptoms, and examined all recent and relevant RCTs between 1997 and 2010, focusing on all cognitive oriented interventions. Nine "well-conducted RCTs" (p. 589) were included in the review, and one meta-analysis of 19 small RCTs, one meta-analysis of six RCTs, and seven RCTs. In contrast to limiting the review to cognitive stimulation, as in Woods et al., this review searched five interventional categories, however, two categories failed to reveal RCT studies (brain training and memory aids). The remaining three categories were included and reviewed. They were cognitive stimulation and reality orientation, cognitive training, and cognitive rehabilitation and implicit learning. Findings revealed that all three therapies provided modest but significant benefit in terms of improved cognitive symptoms in people with $\mathrm{AD}$, which may be further improved in combination with cholinesterase inhibitor therapy. However, the most convincing evidence was for the beneficial effects on cognitive function of CST. This finding is 
comparable to the finding of Woods et al. (2012), and for the convincing evidence for the beneficial effects of cognitive rehabilitation on daily functioning of ADLs. The authors made recommendations for a cost-effectiveness evaluation of cognitive therapies, due the labour intensiveness of these approaches.

Cost evaluation has already begun. Aguirre et al. (2010) concluded that a sevenweek course of CST was both beneficial and cost effective, however, there is less conclusive evidence for effects and cost effectiveness over a longer period. For example, Requenta et al. (2004) conducted a RCT with people with AD, to examine the effectiveness of computer-based cognitive therapeutic strategies in combination with donepezil. The study was resource intensive, because the therapist provided computer aided CST with the groups for 30 minutes a day, Monday to Friday, for one year. Another example of labour intensiveness was the study conducted by Boulay, Benveniste, Boespflug, Jouvelot, and Rigaud (2011) who provided only four computer-assisted interventions on seven people, but had two psychologists and an engineer at each intervention. Currently, many of the cognitive oriented interventions are provided by trained therapists, researchers or professionals, resulting in costly, labour intensive modalities, which are seldom fully implemented in the lived environment (Aguirre et al., 2010; Ballard et al., 2011; George \& Whitehouse, 2011; Woods et al., 2012). In recognition of the labour-intensive issues, Woods et al. (2012) acknowledge that facilitators do not need to be therapists or have professional qualifications, although training for facilitators is key. This view is supported by George and Whitehouse (2011) who have developed a model of 'intergenerational learning', that connects children and others, including PWD, in the teaching and learning process, similar to the intergenerational programmes recommended by Galbraith, Larkin, Moorhouse and Oomen (2015). Also, Greenwood et al. (2016) promote the potentiality of utilising the volunteer workforce, and Astell et al. (2004, 2009, 2010), had success providing cognitive stimulation programmes utilising caregivers.

The review by Ballard et al. (2011) differentiated and clarified a variety of cognitive interventions. However, unlike Woods et al. (2012), the review was brief and some details were missing, for example, the search strategy and databases used. Even so, the review was well presented, coherent, and the findings and recommendations were comparable and generalisable. Both reviews focused on cognitive oriented therapies, within a similar timeframe. Only four studies were common to both reviews (Chapman et 
al., 2004; Onder et al., 2005; Requenta et al.,2004; Spector et al., 2003), which reiterates the methodological limitations of poor description of interventions in dementia care research, resulting in many other studies being excluded due to poorly defined terms, or lack of consistency in the utilisation of validated outcome measures. This gap in knowledge is filled by a third complementary review of the literature.

Oyebode and Parveen (2016) conducted a comprehensive review of the psychosocial interventional literature to purposefully find reports and reviews of highquality that were not covered in other recent reviews. Their extensive search, using multiple databases revealed 61 relevant RCT and controlled studies, between 2008 and 2015. The studies were sorted into four care pathway themes: living at home with dementia, caregiver interventions, interventions in residential care, and end-of-life care. The interventions ${ }^{7}$ in residential care generated the most literature (16 reviews and 12 studies), and were the most relevant literature for this study. Findings revealed that the majority of studies focused on reducing behavioural problems (20), while nine focused on the impact of cognitive interventions on other outcomes such as cognition, everyday functioning, mood, and quality of life. Specific findings included lack of evidence for cognitive benefit from music therapy and physical therapy. However, cognitive rehabilitation techniques, way-finding training, and reminiscence therapy had a positive effect on cognition. Beneficial effects on everyday functioning of ADLs were reported for interventions that were multi-sensory, multi-modal, environmentally based, and activities based on "the needs-driven, dementia compromised approach. The latter approach proposes that people living with dementia have needs in common with those without dementia (e.g., for purposeful activity or emotional security)" (p. 13). Yoga, reminiscence, needs-based interventions, and varied environmental interventions had beneficial effects on mood; however, physical therapy did not. Physical therapy did have beneficial effect on QOL, as did needs-based interventions; however, the evidence for reminiscence therapy was variable for this aspect. In conclusion, the authors recommend that PSI would benefit from moving away from a focus on behaviour control and focus on the wider aspects of life for people living with dementia. This conclusion is supported by Banerjee et al. (2006) and Woods et al. (2012). The review was comprehensive, and

\footnotetext{
${ }^{7}$ Interventions included "staff training, multi-disciplinary meetings and case conferences, manipulation of environmental cues, a wide range of psychosocial and sensory therapies, music therapy and yoga" (Oyebode \& Parveen, 2016, p. 13).
} 
usefully categorised and structured, facilitating reader exposure to relevant high-quality literature.

In summary, all three reviews (Ballard et al., 2011, Oyebode et al., 2016; Woods et al., 2012) found that there was modest but significant evidence that cognitive stimulation consistently benefits cognitive functioning for PWD. The evidence is particularly robust for cognitive stimulation therapy and cognitive rehabilitation. In addition, two of three reviews found that there is evidence that cognitive rehabilitation can substantially benefit everyday functioning (ADLs) for PWD (Ballard et al., 2011, Oyebode et al., 2016). Additionally, Oyebode et al. (2016) identified multi-sensory, multi-model, environmentally and needs based rehabilitation strategies as being the most effective.

This literature review is limited by the plurality of terms used to describe cognitive oriented therapies, as were the above reviews. For example, the terms cognitive stimulation, CST, cognitive training, and cognitive rehabilitation, have been used interchangeably over the years. de Vries et al. (2010) and Woods et al. (2012) report on the hindrance effect of poor descriptions of interventions in dementia care research as a methodological limitation of many studies, including their own. An attempt to define the various cognitive interventions (Clare \& Woods, 2003/2008) has had little impact on how researchers define the focus of the cognitive approach that they take, which makes critical reviews of the literature difficult (Woods et al., 2012). Similar findings were reported by Ballard et al. (2011) and Yamaguchi et al. (2010). This review differentiates between the different approaches of cognitive oriented approaches relative to this study, commencing with reality orientation.

\subsubsection{Reality orientation}

Reality orientation was first described by Taulbee and Folsom (1966), as a technique to improve the QOL for confused older people. It is a cognitive intervention that involves orientation and memory information relating to person, time and place, in an effort to provide a better understanding of the person's surroundings and in so doing, improve the sense of control and self-esteem (Spector, Davies, Woods, \& Orrell, 2000). It can be conducted in one of two settings: in a classroom, in small group meetings during reality-oriented activities; or in a 24 hour a day intervention where caregivers and/or staff 
involve the PWD in reality-based communication at every contact (Spector et al., 2000). Over the years, reality orientation has become less commonly used because the original strategy of engaging healthcare assistants in "a hopeful and therapeutic process... [resulted in a] rigid and confrontational approach" towards PWD (Woods et al., 2012, p. 1). Similar criticism is reported by Kitwood $(1989,1993 b)$ who challenged the need to orientate PWD to our cognitive reality of person, time and place, suggesting that caregivers ought to attune themselves to the PWD's emotional reality. However, 11 years later Spector et al.'s (2000) systematic search and meta-analysis of six RCTs evaluating the effectiveness of classroom reality orientation interventions found the content of reality orientation interventions used in the selected studies included two or more reality orientation strategies ${ }^{8}$. The overall results for the cognition and behaviour were significantly in favour of treatment. In conclusion, the authors recommend that reality orientation techniques should be considered an important component of caring for PWD. They commented that future multi-centre RCTs would be valuable, used in combination with more qualitative studies. Reality orientation is an implicit element of CST.

\subsubsection{Cognitive stimulation therapy}

CST is a group-based approach for providing interventions for people with mild to moderate dementia, based on the theoretical concepts of cognitive stimulation. The first group to develop CST designed a very specific operationalised approach of 14 sessions (bi-weekly), lasting 45 minutes, over a 7-week period, and delivered by a trained therapist (Ballard et al., 2011; Chapman, Weiner, Rackley, Hynan, \& Zientz, 2004; Spector et al., 2003). Participants engaged in a range of activities and discussions aimed at general enhancement of cognitive and social functioning (Cantegreil-Kallen et al., 2009; Spector et al., 2003; Woods, Thorgrimsen, Spector, Royan, \& Orrell, 2006). The initial evidence base for CST in maintaining cognition and psychological wellbeing has its roots in France (Breuil et al., 1994; Moniz-Cook \& Manthorpe, 2009). Early work by de Rotrou, with people with $\mathrm{AD}$, is based on educational neuroscience practised in the United States. The goals are preservation of cognitive functioning, and the increase of self-worth (CantegreilKallen et al., 2009; Moniz-Cook \& Manthorpe, 2009).

\footnotetext{
${ }^{8}$ Intervention included two or more of the following strategies: reality orientation boards, clocks, calendars, maps, object naming, general orientation, and discussion.
} 
A single-blind, multi-centre RCT conducted by Spector et al. (2003) recruited 201 older adults with dementia (158 female and 43 male), (115 treatment/86 control), to test if CST would benefit cognition and QOL. Cognition was measured utilising the MMSE (Folstein, Folstein, \& McHugh, 1975), which is one of the most widely used brief screening instruments for cognition. QOL was measured utilising the Quality of LifeAlzheimer's Disease (QOL-AD) measure (Logsdon, 1996). The 14-session programme ran twice a week for 45 minutes over 7 weeks. The intervention was designed on the principles of reality orientation and cognitive stimulation and comprised of a reality orientation board and topics: using money, the present day, famous faces, and word games. Each session began with a non-cognitive warm-up activity, followed by a themed session that focused on the natural process of reminiscence, with a focus on the current day, and information processing rather than factual knowledge. The facilitator, who was a member of the research team, was able to adapt the level of difficulty of activities depending on the groups' cognitive abilities, gender mix, and interests. The findings revealed that the intervention group had significant improvement over the control group on cognition and QOL measurements. The authors concluded that the results were comparable with the results of drug trials for people with dementia and that CST groups may benefit PWD. Similar findings and conclusions were reported by Ballard et al. (2011), Oyebode et al. (2016) and Woods et al. (2012).

\subsubsection{Cognitive training}

Cognitive training is less specific in methodological approach than CST. It involves teaching theory-based strategies and skills to try to improve cognition and functioning. Participants practise a set of standard tasks designed to reflect particular cognitive functions. A range of difficulty levels may be available within the standard set of tasks to suit the individual's level of capability. Participants are facilitated by a trained facilitator, alone or in groups, and tasks may include pencil and paper or computerised exercises (Ballard et al., 2011; Bellville, 2008).

Kawashima et al. (2005) used brain-training games with people with AD and found modest cognitive benefits can be obtained. This was a small non-randomised quantitative study with 32 subjects, in which 16 experimental and 16 age and MMSE score-matched AD subjects participated in brain training (reading aloud and arithmetic calculations), 2-3 
times a week for 6 months. The results revealed statistically significant differences in the experimental group between the pre-and post-test utilising the Frontal Assessment Battery. Improvements were also found in levels of communication and independence however, there was no change to MMSE scores. The benefits suggest that reading aloud and doing arithmetic calculations can be useful in the rehabilitation of people with dementia. Although this study was not included in Ballard et al.'s (2011) critical review relating to cognitive oriented interventions for people with $\mathrm{AD}$, attention was drawn to it, as it is one of the few brain training game studies to confer modest but significant benefit. Multisensory, enriched environments, such as computer-assisted gaming/exergaming and brain training environments (Khan et al., 2016), is an embryonic but emerging literature.

\subsubsection{Computer brain training and gaming environment}

The computer brain training and gaming environment is a multi-billion-dollar business targeting, primarily, the well population (Ballard et al., 2011; George \& Whitehouse, 2011; Mole, 2016). Many commercial companies have conceived, developed and marketed brain training games based on the concepts of "use it or lose it", which is founded on the principle that specific brain functions will deteriorate over time if they are not utilised (Ballard et al., 2011, p. 590), and "use it and improve it", suggests that intact brain function can be ameliorated through brain training (Gelfand et al., 2014). However, according to Ballard et al. (2011), George and Whitehouse (2011), Mole (2016) and Owen et al. (2010), the scientific evidence of benefit to cognition of brain training games is lacking although modest improvements have been found in older adults and video-game players who perform better on some cognitive tests than non-game players (Owen et al., 2010; Kueider, Parisi, Gross, \& Rebok, 2012).

George and Whitehouse (2011), argue that the brain fitness industry has been stimulated by the void created by failed Alzheimer's disease drugs during the past decade, and are critical of the current "reductionism... of the brain fitness marketplace" (p. 595). However, in 2016, a well-known gaming company agreed to pay US\$2 million to the Federal Trade Commission, an independent agency of the United States government, who charged the company with deceitful advertising and reported that it "preyed on consumers' fears about age-related cognitive decline" (Mole, 2016, p. 2). Mole advocates that brain training companies should "temper their claims" (p. 4), and conduct RCTs, which are 
currently non-existent in research efforts for this industry. Still, George and Whitehouse (2011) conceptualise a contemporary future role for the brain fitness industry, but only by "framing the brain as a community/public health, ethical, and social issue...[focusing on] what it means to protect brains...[in] natural environments that can exert...ameliorative effects on human cognition" (p. 595). There is a large volume of research on assistive technological environments that aim to have ameliorative effects of human cognition and/or wellbeing of people, including sensing, prompting, telecare and assistive living technologies for older people and for people with cognitive impairments (Boger et al., 2016; Davis \& Shenk, 2015a; Favela, 2013; Heyn, Cassidy \& Bodine, 2015; Wherton \& Monk, 2008); computerised cognitive testing for older adults (Inoue et al., 2003; Zygouris $\&$ Tsolaki, 2015); communication and decision making through the use of video and internet (Davis \& Shenk, 2015b; Freeman et al., 2005; White et al., 2002; Zickuhr \& Madden, 2012); and cognitive stimulation and cognitive training for people with brain injury or intellectual and development disabilities and mild cognitive impairment (Dou et al., 2006; Siberski et al., 2015).

More closely aligned with this study, is a sparse literature on computer-assisted exergaming, that evinces that some gaming industry members are moving in the direction suggested by George and Whitehouse, not only in terms of ameliorative effect on human cognition, but also other neurological and physical presentations that are a result of neurological assault, all of which are relative to this study (Im-Able, Ltd., 2013; Jordan, Sampson \& King, 2014; King et al., 2010; King, Hijmans, Sampson, Satherley, \& Hale, 2012; Sampson, Shau, \& King, 2012). Also, researchers are reporting the ameliorative effects of utilising exergaming technology (Galea et al., 2016; Hale et al., 2012; Hijmans et al., 2011; Khan et al., 2016).

\subsubsection{Cognitive rehabilitation through computer-assisted exergaming}

In contrast to the global approach of cognitive stimulation, where both explicit and implicit memory pathways are targeted, cognitive rehabilitation primarily targets the implicit memory pathway and is becoming increasingly popular (Ballard et al., 2011; Oyebode \& Parveen, 2016; Spector et al., 2000; Zanetti et al., 1997). Cognitive rehabilitation is an individualised approach where personally, relevant goals are identified and the therapist works with the person and his/her family to devise strategies to address 
these goals. The emphasis is on improving performance in everyday life rather than on cognitive tests, as in cognitive training, building on the person's strengths and developing ways for compensating for cognitive impairment (Dou, Man, Ou, Zheng, \& Tam, 2006; Ferland, Larente, Rowland, \& Davidson, 2013). A meta-analysis to determine the effectiveness of computerised-cognitive interventions for people with $\mathrm{AD}$ and other dementias found a weak to moderate effect size in PWDs' ability to improve cognitive function (Gelfand et al., 2014). However, cognitive rehabilitation is more aligned to skills and functioning in everyday life, which can be mediated through cognitive rehabilitation (Woods et al., 2012). Furthermore, functioning is seldom the outcome focus of cognitive rehabilitation programmes. Oyebode and Parveen (2016) have called attention to this gap in the literature. Equally important is the aspect of overall QOL. Both of these issues are yet to be appraised through a thorough systematic review of the literature. However, there is an emerging literature that indicates that computer-assisted interventions have some beneficial effects.

Hale et al. (2012) and Hijmans et al. (2011) reported on the mixed methods pilot study, that used a control-washout-intervention design to explore the perceptions of 14 participant experiences using a low-cost hand-held controller (CyWee Z), along with a range of computer games, as an intervention to enhance upper limb function following stroke recovery, and as an adjunct to regular stroke rehabilitation. The games were played with a variety of hand-held devices, either sitting or standing. Participants served as their own control and in the interventional stage played for 45 to 60 minutes, four times a week, for 2.5 weeks. Outcome data were generated through four in-depth interviews, two focus groups and three outcome measures. Thematic analysis revealed that all participants enjoyed computer gaming and there were perceived benefits in upper-limb movements, concentration and balance. The primary outcome measure, the Fugl-Meyer Assessment upper limb section (Fugl-Meyer, Jääskö, Leyman, Olsson, \& Steglind, 1974), revealed significant improvement of upper-limb movement, compared to pre-intervention assessments $(\mathrm{p}<0.001)$. Participants also reported how the device and games could be improved. The authors conclude that using the adapted hand-held controller and computer games in upper-limb rehabilitation is an acceptable and beneficial adjunct to rehabilitation for people with chronic stroke, and appears to be a cost-effective alternative to other forms of therapy. Similar findings are reported by Galea et al. (2016) and Khan et al. (2016). 
During and following completion of the above study, the low-cost interface device was conceived, prototyped and developed to a production ready status as a computerassisted 'exergaming' device, by scientists at Industrial Research Ltd, a NZ Crown research institute, following a four-year process of iterative development, where the games were tested and augmented (Hale et al., 2012; Hijmans et al., 2011; Jordan, Sampson, \& King, 2014; King et al., 2010, 2012; Sampson, Shau, \& King, 2012). The computerassisted exergaming device was commercialised as 'Able-X', a system for the rehabilitation of stroke survivors, by Im-Able Ltd., an independent company in NZ. The Able-X has been recognised with four awards in Australia and NZ (Im-Able Ltd., 2013).

Since commercialisation of the Able-X, it has been utilised in two other research studies. The Able-X was one of three devices used in a larger observational cohort study in Melbourne, Australia (Galea et al., 2016). A clinical practice improvement approach was taken to implement and evaluate a technology-assisted programme of rehabilitation for 92 participants after stroke and other neurological conditions, but not $\mathrm{AD}$ or other dementias. Results showed significant improvement in arm function and strength $(\mathrm{p}<0.001)$, motor function $(\mathrm{p}<0.05)$, functional independence $(\mathrm{p}<0.05)$, as well as QOL and overall health $(\mathrm{p}<0.01)$. The authors concluded that the study "showed promising results for upper limb functioning in persons with neurological disorders", and recommend that the results need to be confirmed with a larger study sample and longer follow up (Galea et al., 2016, p. 522).

In the study by Khan et al. (2016), the Able-X was one of many devices utilised in a RCT, to compare the effectiveness of an environmental enrichment activities programme as an adjunct to usual activities. One hundred and four participants, with neurological conditions, excluding $\mathrm{AD}$ and other dementias, were randomised into an interventional group $(n=53)$ or a control group $(n=51)$. The setting was a publicly funded neurorehabilitation unit. The intervention group were offered individual or communal access to an environmentally enriched "Activities Arcade" (p. 418), which included 10 different activities, four of which were computer-assisted exergaming with Able-X, and three other exergaming/gaming devices: Able-M, Mitii, and Nintendo Wii. Participants also had access to computers, the internet, Skype and a music station. Results showed significant improvement at discharge when compared to controls in depression and stress subscales 
$(\mathrm{p}<0.05)$, cognition $(\mathrm{p}<0.048)$, and functional independence $(\mathrm{p}<0.05)$. Cognitive function and activities improved the most for stroke survivors. Significant differences were maintained at three-month follow-up. The authors concluded that an environmental enrichment activities programme can produce "significant improvement in functional and cognitive ability...compared to routine ward activity programmes" (p. 417), and recommended further research on specific components of the intervention, and more emphasis on outcome-oriented research, that explores the effects of integration of environmental enrichment programmes with current models of rehabilitation. The results on improved functioning were similar to the results obtained by Galea et al. (2016), and the functional ability similar to that found by Hale et al. (2012) and Hijmans et al. (2011).

In advance of the results of the two studies, the approach was made to my primary supervisor, Dr de Vries, by representatives from Im-Able Ltd., to explore interest in further evaluation of the computer-assisted exergaming device 'Able- $\mathrm{X}$ '. There was all round interest in conducting an exploratory study to establish if the Able-X could deliver cognitive, functional and QOL improvements for PWD, a population for which this knowledge does not currently exist. This gap in knowledge became the focus of this thesis. In the absence of a larger volume of literature relating specifically to the evidence of effectiveness for computer-assisted exergaming with Able-X, an aligned literature, that explores more specific cognitive oriented strategies for delivering cognitive stimulation is critically explored.

A smaller body of literature focuses on computer-assisted interventions with PWD (Astell, 2004, 2009, 2010; Bouley et al., 2011; Requenta et al., 2004; Tarraga et al., 2006). One of the most cited studies utilising computers is Tarraga et al. (2006) who conducted a single-blind randomised pilot study to determine the usefulness of an interactive multimedia internet-based system (IMIS) for delivering cognitive stimulation interventions over 24 weeks to 46 mildly impaired people with $\mathrm{AD}$, who were also receiving cholinesterase inhibitors. Participants were divided into three groups. Group one who had IMIS (3 times a week for 24 weeks), along with 3.5 hours a day integrated psycho-stimulation programme had improved cognitive outcomes at 12 weeks (MMSE) and this was maintained at 24-week follow up and beyond. The second group, who only had 3.5 hours a day psycho-stimulation had better cognitive outcomes than the third group (treated with cholinesterase inhibitors only), but the improvements attenuated at 24 weeks. 
This study demonstrated that utilising interactive technology can augment the effects of other more traditional cognitive stimulation programmes and the effects of cholinesterase inhibitors, calling for more research in a less controlled environment. Similar beneficial cognitive findings were reported by Requenta et al. (2004) who conducted a RCT on people with $\mathrm{AD}$ to examine if computer-based cognitive therapeutic strategies, in combination with donepezil, was effective in slowing cognitive decline and improving mood. They found that the combined treatment had a considerable beneficial effect for both cognitive function and mood.

Another important study is the work of Astell et al. (2004), which focused on improving communication for people with dementia, utilising multimedia technology and operating on the 'here and now' principle that does not require remembering previous conversations. Computer-aided reminiscence and communication (CIRCA), is a touch screen operated system containing photographs, music and video clips. Eighteen people with $\mathrm{AD}$ were matched for age, years of education and dementia severity and were assigned to one of two groups: CIRCA or traditional reminiscence therapy. Results revealed that CIRCA facilitated retrieval and conversation and encouraged the caregiver to hand over control of the conversation. Traditional reminiscence were more question and answer sessions where the person with AD played a more passive role. Laughing and humour were coded as evidence of communication and good indicators of pleasure, which is often associated with QOL (MacPhillamy \& Lewinsohn, 1982). Astell et al.'s work creates a bridge between the principles of corporeality and temporality as described by Dewing (2008) and relational models of care as described by Morhardt and Spira (2013) and Nolan, Davies and Brown (2006b). Astell et al.'s CIRCA has only been tested using carers in residential settings. It is possible that the same effects would be forthcoming within a relational model of care in the community however, as it currently uses a caregiver/therapist model, it will have resource limitations.

Even when the focus is on the efficacy of the computer-assisted technology, perceived benefits of this modality of intervention surfaced, as demonstrated by Boulay et al. (2011), who conducted a usability study where the MINWii (music therapy game), along with a Wiimote Pistol were used to mix music therapy and cognitive stimulation via video gaming technology (MINWii). Seven people with mild to moderately severe AD were included in the study. The automatically computed results show participants progress 
in terms of "instant mastery or a clear learning effect" (p. 233). Although no outcome measures were used, the authors found that the MINWii fostered reminiscence and positive interactions with caregivers, even in patients with moderately severe AD. In addition, patients enjoyed and wished to repeat the experience. Similar reports of improved relations with caregivers and enjoyment relating to computer-assisted interventions for PWD were found by Astell $(2004,2009,2010)$ and Hale et al. (2012).

Improved relationships and enjoyment relate to the benefits that can be realised from environmental enrichment, as explored by Khan et al. (2016). Yamaguchi et al. (2010) concur and add the environment is enhanced through respectful communication and reasons that non-pharmacological intervention therapy is different in every situational case and results are dependent on all present, including the researcher/therapist. Astell (2004) had earlier argued that successful psychosocial interventions therapy has at its core how the therapist communicates with the PWD and not what approach is taken, as demonstrated in the rise, fall and recent resurrection of reality orientation interventions for PWD (Spector et al., 2000; Woods et al., 2012).

In addition to how the therapist communicates, Strack, Martin and Stepper (1988) argue that people can conjure affective emotions in others, for example facial feedback theory demonstrates that a smile, when perceived by others, results in spontaneous activation of the muscles related to smiling in the observer, evoking a pleasant feeling as an automatic, rather than a cognitive reaction. Astell (2004) was the only researcher to code laughing and humour as evidence of communication and indicators of pleasure. However, self-reported perceived enjoyment relating to computer-assisted interventions for PWD were found by Boulay et al. (2011) and Hale et al. (2012), which according to MacPhillamy and Lewinsohn (1982) are often associated with QOL for PWD, highlighting the potentiality of the effects of communication, by the therapist/researcher, both verbal and non-verbal, when providing psychosocial interventions to PWD. The following section of this review provides an in-depth overview of the theoretical basis for one of the most effective forms of cognitive rehabilitation that utilises the principle and strategies of EL with PWD, that were key influences in this study. 


\subsubsection{Cognitive rehabilitation through errorless learning}

The conceptual foundation for EL is a behavioural theory conceived by Skinner in the early 1930s which was applied and advanced by his students, including Terrace (1963), through his 'learning with and without errors work'. According to Terrace, a simple discrimination learning procedure is one to which the subject learns to respond. For example, in his seminal work, pigeons were trained to peck at a red light and were rewarded for 'correct behaviour'. No reward was given for pecking at a green light, 'errorful behaviour'. Terrace had four groups of three pigeons, all male: Early Progressive, Early Constant, Late Progressive, and Late Constant. For the Early Progressive group (learning without errors group), errorful discrimination was faded in over the first three sessions and was constant by session four. The sequential fading-in commenced as a black stimulus lasting a few seconds and then gradually faded-to a fully saturated green light. Out of three subjects, the range of error responses to errorful discrimination was 5-9. In contrast, the group of pigeons that learnt the same discrimination without fading-in procedures and where errorful discrimination was introduced much later, in session 22, of the conditioning process (Late Constant group) the range of errors was 1922-4153. The other two groups fell between. The Early Constant group range of errors was 191-210, and the Late Progressive group range was 31-760 in the first three discrimination sessions.

These results provided evidence that discrimination learning without errors can be acquired, but learning is dependent upon the manner in which the discrimination is introduced. Terrace (1963, p. 13 \& p. 25) found that the birds that learned without errors were not upset by the errorful green key, they calmly and quietly waited for the correct key to appear. In contrast, birds in the other groups were agitated when the errorful green key appeared, they would "usually flap their wings, stamp on the floor...and orientate themselves away from the key", implying that during the process of error extinction, the error key acquires aversive properties to which birds acquire a fright reaction that occasionally resulted in an outburst of responses to the errorful key. Terrace found that learning could occur without errors when training began early in operant conditioning and when fading procedures were in place. The birds that learned without errors did not display the agitation, fright reaction, or emotional and aversive behaviours that were seen in all the other groups, they calmly and quietly waited for their reward. Terrace's theory, that discrimination learning without errors can be acquired, challenged the accepted epistemology of the day regarding discrimination learning, that extinction of responding 
to error key (trial and error or learning by mistakes) was considered necessary (Evans et al., 2000; Keller \& Schoenfeld, 1950).

The evidence from Terrace's work is convincing. The study and findings are logically presented and his results are widely generalisable as demonstrated through application to human subjects. It is over 50 years since Terrace conducted his study, and his fading techniques were proved successful in training children (Schreibman \& Koegel, 1981). Terrace (1981) recounted that Skinner, his academic advisor, "cited his [Terrace's] dissertation results as evidence for the efficacy of programmed instruction" (p. 123), a step-by-step procedure for providing EL opportunities by teaching all the prerequisites that are necessary for the desired learning to take place (Sidman, 2010). The following is an example of programmed instruction called backchaining, an EL technique that is particularly effective in the development of behavioural and functional skills:

The teacher would start by tying the shoe almost completely, asking the child only to complete the job by pulling the final loops tight. This simple act would produce immediate and substantial reinforcement [reward] for the child....Then, the teacher would untie the shoe and tie it again, but this time, not quite so far.... The teacher would gradually work backward. On completing each new small step, the child would always be in a position to do what she had already learned and would be able to go through the rest of the sequence successfully. (Sidman, 2010, p. 172)

Sidman (2010) indicated other behavioural instances where backchaining would be effective, including ADLs: dressing, making a bed, washing hands, brushing teeth and self-feeding. Although children are the focus in the examples above, this step-by-step EL process has also been shown to be successful with older adults, especially PWD (de Werd, Boelen, Olde Rikkert, \& Kessels, 2013, 2015; Schmitz et al., 2014; Thivierge, Simard, Jean, \& Grandmaison, 2008; van Tilborg, Kessels, \& Hulstijn, 2011).

Over the last 20 years, EL concepts have been adapted to areas of adult rehabilitation and implicit learning, including basic skills of ADLs, and the more complex instrumental activities of daily living (IADLs). Some procedural memory deficits can be seen well in advance of symptomatic presentation, for example, Pérès et al. (2008), who conducted a 10-year prospective study, found people had at least two IADLs (e.g. 
telephoning, transportation, medications, finances) deficits 10 years before the clinical diagnosis of dementia was made. Similarly, Lau, Parikh, Harvey, Huang and Farias (2015) conducted a longitudinal study (4.1years) involving people who were cognitively normal or diagnosed with mild cognitive impairment, and found those with mild changes in functional abilities at baseline assessment had a four-fold risk of developing IADL disabilities in the future. They articulated the importance of developing interventions to support every day abilities in an effort to delay loss of independence.

There are other EL techniques that incorporate elements of programmed instruction and backchaining. According to de Werd et al. $(2013,2015)$ and Ballard et al. (2011) the most beneficial technique is self-generated EL, which involves several strategies: teaching techniques aimed at reducing the number of errors during acquisition of learning, effortful learning, small customised steps, verbal instruction and use of vanishing cues (a signal that tells you to begin or end an action); which is similar in method to programmed instruction (Terrace, 1963), and backchaining (Sidman, 2010).

There is also embryonic evidence that it is possible to reteach lost skills as well as new tasks to PWD using EL techniques. Re-learning, referred to as 'rementia' by Kitwood (1989, 1997) and Sixsmith, Stilwell and Copeland (1993), involves some lost cognitive and/or functional skills being regained through appropriate social, care and interventional contexts. For instance, Thivierge et al. (2008) used EL and spaced-retrieval implicit memory techniques to facilitate the relearning of IADLs in two people with AD. One participant was trained to relearn the use of voice mail and the other to use an answering machine. Both had improved performance, which was maintained over a five-week period, demonstrating the primary efficacy of EL and spaced-retrieval techniques, especially when tailored to the needs of the person. Likewise, van Tilborg et al. (2011) found that older people with mild dementia were able to learn new skills such as using a microwave oven and a coffee machine. The group who were trained using the implicit technique of modelling (observation and imitation) and the group who were trained with the explicit technique of providing verbal cues had similar rates of learning; the key being to avoid errors during the learning process. In addition to relearning and learning, Schmitz et al. (2014) found that EL approaches allowed for faster reaction times than errorful approaches in people with AD and healthy others. 
Other areas in which EL concepts have been adopted include assisting learning in people with acquired brain injury and for individuals with intellectual and developmental disabilities. In a single case quasi-experimental study, Ferland et al. (2013) found that rehabilitation using EL techniques during stroke recovery in an individual who had severe memory and initiation deficit, to be beneficial. The individual learned new routines, which were maintained at three months. Similarly, in a quasi-experimental clinical design study, involving 37 patients who were randomly assigned to one of three groups, Dou et al. (2006) found that people with brain injury performed better than a control group where one group received an intervention utilising a computerised EL-based memory rehabilitation and the second group attended a therapist-assisted memory group. Findings indicated that people with brain injury in both groups performed better than controls on cognitive tests and behavioural tests, although no differences were found between the intervention groups.

Finally, but not exhaustively, EL techniques have been applied to cognitive and memory problems in PWD (Clare et al., 2000; Clare \& Jones, 2008; Clare \& Wilson, 2004; Clare, \& Woods, 2003/2008; Laffan, Metzler-Baddeley, Walker, \& Jones, 2010; MetzlerBaddeley \& Snowden, 2005). This application is presently the most prolific research area relating to dementia and EL. Clare et al. (2000) were the first to report examining EL in relation to dementia of the Alzheimer's type. In their multiple single-case experimental design study, interventions focused on every day memory problems (learning face-name associations or improving recall of personal information, learning to use memory aids), and were based on the EL principle and a selection of three or more EL techniques: verbal elaboration, vanishing cues, cueing, expanding rehearsal, use of photographs, supervised practice in the real-life setting, mnemonics, structured practice using audiotape, prompting, electronic diaries, and using a memory board. The results demonstrate significant improvement on specific everyday memory tasks for five of the six participants and this improvement was maintained for up to six months. There was no significant change in the neuropsychological profile at post intervention testing and at 6-month follow up.

Although supportive of EL techniques, Jones and Eayrs (1992), Clare and Wilson (2004), and Ferland et al. (2013) recommend that when using EL approaches, it is important to select tasks that can be taught that way. In contrast, Banerjee et al. (2006) recommend that researchers extend beyond discrete functions, arguing that "reliance on 
measures of discrete functions, such as cognition, could lead to the positive effects of interventions being overlooked or to potential negative effects of intervention being missed" (p. 148), a view supported by Oyedobe and Parveen, (2016). Conversely, Woods et al. (2012) argue that other secondary benefits, including QOL and wellbeing, are mediated through improved cognition. Therefore, while the majority of studies involving participants with mild cognitive impairment and mild to moderate dementia, focus on the aspect of cognition alone, the key is to build in objective evaluation of a wider scope of outcomes, including QOL and wellbeing. Oyebode and Parveen (2016) concur, as do Yamaguchi et al. (2010), who offer another very important QOL aspect that is promoted and protected through EL interventional strategies but rarely measured: the aspect of 'personal dignity'. Personal dignity can be assured, if care is taken to make certain that the PWD succeeds, and if errors are made, that the errors are not pointed out. In addition, the environment should be conducive to both PWD and the delivery of the intervention (Yamaguchi et al., 2010); in other words, the environment should be enriched (Astell, 2004; Enosh \& Ben-Ari, 2016; George \& Whitehouse, 2011; Khan et al., 2016; Oyedobe \& Parveen, 2016; Prince et al., 2014).

This segment of the literature review demonstrates that implicit memory pathway is relatively spared in dementia. However, errors made during learning are strengthened in implicit memory, through the priming effect upon subsequent experience, thought or action. Therefore, learning is more efficient if errors can be avoided during the learning and relearning process. The most beneficial techniques for achieving this are EL techniques that are aimed at reducing the number of errors during learning acquisition. These include 'self-generated EL' and programmed instruction and backchaining that incorporate effortful learning, small-customised steps, verbal instruction, and vanishing cues. In section 2.3, I critically review 'personhood' and person-centred care, to highlight the importance of social and relational environments when conducting research of this type.

\subsection{Personhood and person-centred care}

The concept of personhood in the field of dementia was developed by Kitwood in the 1980s (Kitwood, 1989, 1990, 1993a, b, 1997). Personhood is defined as "a standing or status bestowed upon one human being, by others, in the context of relationship and 
social being" (1997, p. 8). Kitwood presents three important philosophical discourses that help to explain the positioning of personhood and how he arrived at the definition used in his work. These are the discourses of transcendence, ethics, and social psychology. Transcendence discourse relates to the sacredness of existence and being, and that "life is to be revered." Ethical discourse conveys our ethical and moral framework when dealing with others, that determines that each person has "absolute value", and should be treated with "deep respect", and social psychology discourse, is associated with "self-esteem...the place of the individual in a social group...performance of given roles and with integrity, continuity and stability of sense of self' (1997, p. 8).

Kitwood's model of personhood is rooted in the tradition of symbolic interactionism in that personhood is emergent only through appropriate social relationships, within an environment free of malignant social psychology (Kitwood, 1990, 1993a, b, 1997). Therefore, failure to attend to the social psychology of relationships and the environment could result in 'the realm of 'unbeing' a person, therefore loss of personhood", or loss of 'self' (1997 p. 46) due to the potential for accelerated cognitive decline. In identifying the link between cognitive decline and loss of personhood resulting in the 'realm of unbeing', Kitwood discloses his Cartesian views relating to mind-body dualism and symbolic interactionist ontology; a position that is critiqued and questioned by others (Dewing, 2008; Kontos, 2003, 2004, 2005; Sabat, 2001, 2014). Additionally, Kitwood (1993a, 1997) advocates for 'continuity and stability of sense of self', however, others claim this is not a realistic outcome when living and dying with dementia (Beard, 2004; Dewing, 2008; Millett; 2011 Pearce et al., 2002).

Dewing (2008) asserts that a more contemporary view of personhood should consider the concepts of body and time (corporeality and temporality), concepts that are missing from the writings of Kitwood. This view is endorsed by Pearce et al. (2002) who affirms the need to re-construct self through constant re-appraisal of self as the disease progresses over time. Similar views are held by Beard (2004), who concludes that PWD make sense of their lives and they "preserve themselves" (p. 415), by managing their interactions with others. There is an over reliance in the literature of the concept that PWD maintain 'self' and 'selfhood', suggesting that this concept places unnecessary burden on unpaid caregivers and families, who are denied opportunity to mourn the loss of the person they had known, and who may feel guilt or shame about their feelings towards the 
'changed' person (Millett, 2011). Millett argues that if we take the view that a person with dementia is in the process of losing their self, the end-point is "a non-person... an ontological null-point" (p. 1). However, Kitwood's philosophy of personhood revolutionised thinking and action from a biomedical model of finding a cure for the diseased brain, to one that put the whole physical and social person into the framework of caring for PWD, a model of care that he called person-centred care (PCC), which is considered to be the cornerstone of caring for older people and PWD (Brooker, 2004, 2007; Dewing, 2008).

Brooker (2004, 2007) was particularly influential in moving the concepts personhood and PCC forward after Kitwood, through the VIPS framework, a framework to assist the implementation of PCC practices. VIPS is an acronym for "Value base that asserts the absolute value of all human lives regardless of age or cognitive ability. An Individualised approach, recognising uniqueness. Understanding the world from the Perspective of the service user, [and] providing a Social environment that supports psychological needs" (2007, p. 13). Implementation of the VIPS framework in practice has been used by a number of practitioners, particularly to design programmes of training for staff in long-term care facilities (Passalacqua \& Harwood, 2012). VIPS has demonstrated QOL outcomes for residents, including reduced care depersonalisation, increased empathy, hope, and concrete communication strategies by caregivers. More recently, emerging from the VIPS framework, is the VIPS practice model, which facilitates implementation of VIPS and is used to facilitate reflection on interactions with PWD and their families; it incorporates a training and coaching approach and is underpinned by organisational change theory (Røsvik et al., 2011, 2013).

PCC, and by association VIPS, continues to have its critics. Dewing (2008) asserts that Kitwood's concept of personhood and emergent PCC programmes continue to be viewed as 'dualist' due to their Cartesian roots of mind and body having the ability to be separate, therefore stigmatising and polarising the physical body of the PWD and their brain or mind, and treating them as separate entities. Others are also beginning to challenge PCC as supporting polarised or asymmetrical models of care, where the PWD is a separate entity from family members and caregivers, despite the social context within which they are being considered (Dewing, 2008; Morhardt \& Spira, 2013; Nolan et al., 2006b; Nolan, Ryan, Enderby, \& Reid, 2002), or because the current PCC model treats 
them as passive, rather than active agents (Kontos, 2005). Forbat (2003) concludes that some PCC models fail to explore the more complex positional relationships, a view that is supported by de Vries et al. (2010), who advocated for urgent integration of the perspectives of the PWD and their family and caregivers to both improve understanding and to factor these complexities into care interventions. Moreover, integrating the perspectives of the PWD and their family and caregivers has been shown extend the independence of PWD and delay admission into residential care setting (Alzheimers New Zealand Incorporated, 2008; Banerjee \& Wittenberg, 2009; Boustani et al., 2003b; de Vries et al., 2010; Mittelman et al., 2004). The VIPS work of Brooker $(2004,2007)$ and her co-workers (Passalacqua \& Harwood, 2012; Røsvik et al., 2011, 2013) continues to advance in line with contemporary theory, especially in terms of ensuring engagement, training, and communication with family and caregivers as they proceed into the PCC pathway for PWD. However, Mitchell and Agnelli (2015) conclude by upholding the work of Kitwood in preference to that of Brooker (2007) and Nolan et al. (2006b), as being easy to relate to and defining what is good, or conversely, unintentionally bad about practice.

Similarly, Dewing (2008) advises caution regarding shaking the foundations of PCC practices, concluding that the foundations are solid, and that Cartesian mind-body duality argument, stigmatisation and polarisation can be addressed through the implementation of alternative models of care and contemporary theory and upholds the work of Kontos as offering a contemporary conceptual framework for understanding dementia from a position of 'embodied selfhood', as an alternative lens to Kitwood's 'Personhood'.

There is an emergence of 'relational' focused or 'family' focused models of care (Brooker, 2003, 2007; Mitchell \& Agnelli, 2015; Morhardt \& Spira, 2013; Nolan et al., 2006b), where the PWD and their family member and/or caregiver are seen as a dyad in all treatment choices, decision making and care interventions. Similar relational family/whānau models of care were the norm for Māori prior to mass urbanisation, when Māori lived in rural communal living spaces (Marae) and where individuals with an illness or disability, including dementia, were cared for within a whānau/family model of care, often without outside assistance. Since the mid-20 th century, more Māori have moved away from the Marae and the whānau model has decreased, with a resulting increase in 
reliance on public health services (Martin \& Paki, 2012). There is evidence of a significant increase in Māori participation in dementia services through culturally sensitive and inclusive models of service development and delivery (Martin \& Paki, 2012).

For this thesis, I have selected the work of Kontos as offering a theoretical and analytic framework and contemporary alternative to Kitwood's personhood. I challenge symbolic interactionist and Cartesian ontological concepts that underpin the notion that 'personhood' (self) can be lost due to brain pathology and absence of another person to facilitate the continuance of self.

\subsection{Kontos: Embodied selfhood}

Pia Kontos is an established contemporary researcher whose research interests include the use of critical social theory and qualitative research methods to examine care practices in long-term care and rehabilitation facilities such as clinical care practices for PWD (UHN, 2015). She advocates an approach to PCC that philosophises the importance of movements and gestures of the body, and primary socialisation, as sources of selfexpression, which she calls embodied selfhood (Kontos, 2003, 2004, 2005, 2012, 2014; Kontos \& Martin, 2013; Kontos \& Naglie, 2007a, b, 2009).

Kontos (2003) argues that "what it is to be human" (p. 159) in the western healthcare system is overlooked when individuals with dementia are viewed through a biomedical and often Cartesian lens. This lens categorises individuals in terms of cognitive deficits and other biological factors in an attempt to understand the pathophysiology of the brain, often to the exclusion of important psychological and social factors. In so doing, there is a constant search within the biomedical field of healthcare research for new pharmacological solutions to cure, treat and prevent, or at least modify, the neurodegenerative process (Birks, 2006; Brickell, 2012; McShane et al., 2006; NICESCIE, 2006/2015; Sabat, 2014). The biomedical approach to dementia assumes that many of the symptoms of dementia are caused by neuropathology and therefore can be treated using pharmacological approaches (Birks, 2006; Brickell, 2012; McShane et al., 2006; NICE-SCIE, 2006/2015). This view is challenged by contemporary scholars (Brooker, 2001, 2004, 2007; Dewing, 2008; Hansen et al., 2008; Kitwood, 1989, 1990, 1993a, b, 1997; Kontos 2003, 2004, 2005; Røsvik et al., 2011, 2013; Sabat, 2014). 
The basis of Kontos' argument theoretically challenges the epistemology and ontology of Cartesian philosophy within the context of dementia health and social care that is currently dominant in western healthcare systems and dementia care practices. She advocates that the discourse on AD must "embrace the facticity of our embodiment...in contrast to mind-body dualism" (Kontos 2003, p. 160), the latter of which has influenced many scholars (Cohen \& Eisdorfer, 1986; Davis, 2004; Fontana \& Smith, 1989; Kitwood, 1997). She proposes a complementary approach to the personhood model of care proposed by Kitwood (1997). Kontos' $(2004,2005)$ champions an approach that respects PWD as 'embodied beings', and she constructed an approach to 'embodied selfhood' that values the body as a source of self and not only a product of a self that resides in the mind as it is in Cartesian duality and other models of care that are influenced by this ontology.

Kontos (2004) conducted a robust ethnographic study of selfhood in Alzheimer's disease, engaging 13 participants ( 3 men, 10 women) who resided in an Orthodox Jewish long-term care facility in Ontario, Canada. Their cognition ranged from moderate to severe (score 1-15) according to the Mini-Mental State Examination (MMSE) (Folstein, Folstein, \& McHugh, 1975). Eight participants had moderate cognitive impairment and five were severely impaired. Proxy consent was obtained because of cognitive impairment. Participant observation was conducted over eight months, for eight hours a day, three days a week. Observations took place in a variety of social situations: scheduled activities programmes, holidays, celebrations, meal times, and during unscheduled activities such as walking around or sitting in the lounge. Detailed and descriptive field notes were recorded during the observations, which focused on the bodily movements and sociability of her participants. Data were thematically organised into five categories: appearance, social etiquette, caring, dancing, and gestural communication. To facilitate analysis of the data, Kontos constructed a conceptual framework that drew on the notion of "intentionality of the body" (Merleau-Ponty, 2014, p. 139), and Bourdieu's (1977) concept of habitus. In doing so, Kontos evinces the existence of embodied selfhood, and challenges the Cartesian epistemology of mind-body dualism that has influenced approaches to dementia care, including the most recognised approaches of personhood (Kitwood, 1997) and person-centred care (PCC) (Brooker, 2001, 2004, 2007; Kitwood, 1997; Kontos, 2005). 
According to Merleau-Ponty (2014), the body has the capacity to perceive and experience the world through bodily agency, practical involvement and a natural affinity with the environment. Bodily intentionality is attributed to pre-reflective (primordial) actions of the body, including bodily movements, gestures and how the body interacts with and utilises the environment, as opposed to being attributed to cognition. Merleau-Ponty philosophises, "I am of space and of time... [and] my body fits itself to them and embraces them" (p. 141), and argues "a movement is learned when the body has understood it and incorporated it into its world" (p. 140). By this, he is referring to the habitual body, which recognises and understands movement. In so doing, the movement participates with one's own body schema. It is through this process of habit and habitual sentient experiences, that we are able to "dilate our being in the world...[or alter] our existence through incorporating new instruments" (p. 145). Therefore, reiterating that purposeful movement of the body does not require conscious thought, it is rather, 'I can', in contrast to, 'I think' (Merleau-Ponty, 2014). In other words, human beings have a hold on their body through the perceptual significance by which the experienced and experiencing body has agency of action. This agency is brought about by primordial and habitual sentient experiences of the body that allow a person to apprehend and convey meaning. In addition, as humans are of space (spatiality) and of time (temporality), the space occupied by one's own body (corporeal) may alter as other objects, such as habits (manners, use of cutlery), or instruments (walking stick, walking frame or wheelchair) become part of our body schema. For example:

If I possess the habit of driving a car, then I enter into a lane and see that 'I can pass' without comparing the width of the lane to the fender, just as I go through a door without comparing the width of the door to that of my body. (Merleau-Ponty, 2014, p. 144)

The above example demonstrates non-cognitive intentionality of the body, a body that perceived and interacted with the environment without thinking or measuring the dimensions of the space to be occupied. It was already known, or perceived by the body in the car, and the body approaching the doorway, that the space to be occupied was large enough. This embodied perceptual knowledge comes from primordial and habitual experiences of being-in-the-world and interacting with the environment, and exemplifies 
how the body has a hold on itself, and a hold on the environment with which it interacts (Merleau-Ponty, 2014).

Kontos (2004) articulates the notion of "embodied selfhood" as "the complex interrelationship between primordial and social characteristics of the body, all of which reside below the threshold of cognition, are grounded in the pre-reflective level of experience, and are manifest primarily in corporeal ways [movement and gestures]" (p. 837). She exemplifies intentional body movement and expanded bodily schema (a pearl necklace) as follows:

When Molly reached behind her neck to pull her pearls from beneath her bib, she 'knew' where her hand was, and how far and at what angle she had to reach to grasp the pearls...[intentional of] a certain outcome by...[her] actions, and the enabling actions were spontaneously distributed amongst the appropriate parts of...[her] body. (Kontos, 2004, p. 838)

Kontos illustrates 'gestural communication' as another example of the intentional body and bodily manifestation of selfhood through the following experiences:

Abe sat down in the dining room and shouted 'bupalupah'. Anna twisted around in her chair so that she could see Abe (his table was behind hers). Abe's face opened up. His eyes grew wider, his mouth eased into a broad smile and he shouted 'brrrrrrr' with first rising and then falling pitch. Anna imitated him, and shouted back 'brrrrrrr' with the same changes in pitch....Anna eventually turned around in her chair to be seated with her back to Abe. Abe shouted 'bupalupah', as if wanting to initiate another exchange, but Anna instead raised her arm above her head and then swiftly dropped it with a sharp flick of her wrist. With this gesture, she terminated the interaction and they each began to eat their breakfast quietly. (Kontos, 2004, p. 836)

The above example demonstrates many aspects of gestural communication, including posture, body movement, facial expressions, eye behaviour and imitation, as well as the pitch and tone of the voices and verbal pauses as communicators await their turn. 
Kontos (2003, p. 161) argues that the "individualistic nature of phenomenology [after Merleau-Ponty] prevents it from exploring how bodies are always socially formed and located" (p. 161). In an effort to socially locate the body, Kontos (2004) draws on a second theory, Bourdieu's (1977, 1990) concept of habitus. Kontos (2003, p. 162) interprets Bourdieu's analysis of the practical logic of the cultural and social practices regarding human behaviour or habitus as "the source of an individual's understanding of the pattern of how things are usually done". In other words, the individual is agent in the social situation with evolving roles and relationships, and each agent is a "producer and reproducer of objective meaning" (Bourdieu, 1977, p. 79). As the individual agent accommodates to his/her role and to the relationships in the context of his/her position in the social field, he/she internalises the relationships and expectations for operating (being agent) and over time, these habitual expectations and relationships form the habitus (Bourdieu, 1977, 1990).

The concept that the external world is imprinted on the body is contested because this infers that the body is a "mechanical system" affected by the external world of which it is part as an observer rather than a sensing body that has "knowledge in the virtue of its always being with us" (Flynn, 2011, 3, ף5). The former view is similar to intuit or instinctive associative memory and mechanistic actions referred to by Descartes (1993), and the "inner resources" referred to by Kitwood (1993b, p. 62). Kontos deals with this notion by distinguishing between two sources of embodied selfhood. The first emanates from the pre-reflective corporeal dimensions of the pre-reflective body and the second from the socio-cultural dimension of the pre-reflective body.

Kontos (2004) interprets habitus as functioning "below the threshold of cognition...and language...enacted at a pre-reflective level...beyond the reach of introspective scrutiny or control by the will" (p. 841). She argues that in contrast to, but complementing the primordial individualistic embodied selfhood, after Merleau-Ponty, the body also has habitus that manifests as a more relational or social embodied selfhood, which is demonstrated in socio-cultural ways of being-in-the-world. Kontos' interpretation of Bourdieu's habitus has similar character to Merleau-Ponty's sentient intentional body, Kitwood's “enduring personality patterns" (1993b, p. 543), and Descartes' (1993) mechanistic associative body, that draws on special modes of thinking such as imagination and sensing, which Descartes reasons to be corporeal extended 
thinking substances. However, Kontos extends her options for data analysis within a cultural and social dimension by applying the notion of habitus after Bourdieu (1990, 1977).

Examples of how residents in the dining room knew instinctively how to use the utensils, condiments, and napkins are provided by Kontos (2004). She explains embodied selfhood is demonstrated by how they ate their meals, respected table etiquette, and socialised together. She argues that primary cultural and social experiences are embodied, and materialise in our habitual state, as demonstrated by how we act. The behaviour of residents, she claims, is not dictated by rules and regulations of the establishment, "but because the taken-for-granted, pre-reflective nature of their practices flowed from embodied selfhood...[or] mastery of a common code" (p. 842). Bourdieu (1977) reiterates that the habitus is "laid down in each agent by his earliest upbringing, which is the precondition for...co-ordination of practices... [and] practices of co-ordination” (p. 81). This is exemplified by Kontos below:

Bertha, the study participant with the most severe cognitive impairment, always said "thank-you" when her private sitter wiped food from her mouth or chin....Abe belched very loudly in the dining room. (Kontos, 2004, p. 833)

Anna, who was seated just one table over, held her hands over her ears and shouted, 'I can't take that. Tell the messhuggener [Yiddish for crazy person] to stop it.' (Kontos, 2004, p. 833)

The first example demonstrates embodied social etiquette and the second, exemplifies "strong reactions to those who were not well mannered" (Kontos, 2004, p. 833). In these examples, Kontos aims to demonstrate that the residents are engaged and interacting with coherence, purpose and meaning through their own powers of cultural and social agency, supporting her concept of embodied selfhood. She argues that participants in her study were "actively involved...through their embodied way of being-in-theworld...Interacting meaningfully through activity and engagement rather than contemplation and reflection", thus reinforcing the pre-reflective nature of embodied selfhood and the importance of social interaction (p. 831). Her central claim is that selfhood is "a fundamental level of [embodied] existence that does not involve cognitive 
consciousness" (Kontos, 2004, p. 837), and resides in the pre-reflective body. Embodied selfhood emanates from two sources, the first being "the body's power of natural expression...[which] manifests in the body's inherent ability to apprehend and convey meaning...such as basic bodily movement, gestures, and sociability" (p. 837). The second source is the "socio-cultural dimension of the pre-reflective body...[for example] mastery of socio-cultural practices (p. 837)...[with] spontaneity and improvisation” (p. 845), similar to the priming effect of the implicit memory pathway (Kihlstrom et al., 2007). Kontos reasons that PWD retain "life-force...that continues to engage with the world...despite the loss of memory" (Kontos 2003, p. 161), reiterating Merleau-Ponty's philosophy of 'I can' in contrast to 'I think' (Merleau-Ponty, 2014).

Kontos' embodied selfhood is a new approach that shifts the focus in the discourse surrounding dementia, away from the Cartesian concept of mind-body dualism and loss of self. In the emergence of embodied selfhood, she acknowledges the seminal work of Kitwood (1997) as being influential in terms of emerging and contemporary approaches to dementia care, including her own (Kontos, 2003, 2005). Kontos' (2004) ethnographic study is complex, but she develops, justifies, and applies her embodied selfhood analytical model to facilitate interpretation of her data, which is done in detail, providing enough description for others to follow. Dewing (2008) and Twigg (2010) uphold the work of Kontos as offering a contemporary conceptual framework for understanding dementia and advancing Kitwood's concepts of personhood and PCC.

In contrast, although complimentary of Kontos' "beautiful observations", Millett (2011, p. 6), critiques the work of Kontos through the lens of bio-phenomenology and contends that the concepts of umwelt and bio-semiosis would be a more appropriate narrative, in that they shift the focus away from 'self' and 'selfhood' and therefore its association with Cartesian philosophy. Millett argues that all animals, including human beings, create an umwelt, or meaningful surrounding world, through the interaction of their sensory receptors and the physical world around them" (p. 8) through bio-semiosis. He asserts that this narrative presents a case for understanding how individuals continue to “engage with life and co-create a [meaningful] life-world... (p. 6), and "inner life...[that is] the basis of a value that people have that is independent of what others think or feel and independent of cognitive capacity" (p. 2). Moreover, by understanding the PWD from the inside out, caregivers and family members would be more sympathetic and able to "act in 
the interests of the individual with dementia, and provide sympathetic care" (p. 12). Millett justifies this position by associating Kontos' embodied selfhood with the Cartesian perspective of over reliance on the concept of self as in selfhood. However, Millett acknowledges that Kontos' work on embodied selfhood has moved the discourse forward by countering the belief that loss of self is the result of loss of cognitive capacity.

The ideas of Kontos and Millett are not so different, in that they both explore how the very essence of life of each individual with dementia continues to reside within the physical body through the human process of being present in and interacting with the world in any given time and space. However, through the ethnographic lens of Kontos, we are able to see the autonomous embodied self through intentional bodily movement, gestures, sociability, and socio-cultural dimension of the pre-reflective body. In contrast, through the lens of bio-phenomenology of Millett, the focus is on understanding the inner-life of the individual with dementia from a caregiver perspective, the inference being on dependency of the individual with dementia on others, rather than celebrating embodied autonomy. A similar view is expressed by Oyebode and Parveen, (2016) whose views are underpinned by a bio-psycho-social model of dementia care, where embodiment is in relationships and social networks, external to the PWD.

Although well cited in the literature I only found one article that presented a summary of Kontos' analytical model when applied to how we dress. Twigg (2010) articulates how clothing forms corporeal extensions related to habitual, social, and cultural embodiment, arguing that "we occupy our clothes, and these, rather than our bodies, are what we are primarily viewed on...[indicative of]... a class and symbolic differentials that categorise and rank its relation to other classes" (p. 225), a concept highlighted by Kontos (2003, 2004, 2005). It is difficult to know how Kontos' $(2003,2004)$ analytical model of embodied selfhood will be interpreted and applied by others. Kontos continues to develop her framework (Kontos, 2005, 2012, 2014; Kontos, \& Martin, 2013; Kontos, \& Naglie, 2009).

Kontos' (2003) approach embraces both the ethical and social psychology discourse narrated by Kitwood. However, their philosophical underpinnings contrast. Kitwood's model of personhood is influenced by the tradition of symbolic interactionism in that personhood is emergent only through appropriate social relationships within an 
environment free of, malignant social psychology (Kitwood, 1990, 1993a, b, 1997). In contrast, Kontos' ontological assumption is that the body is agential at a pre-reflective level and no matter how ravished the brain is due to dementia or disease, selfhood is never lost as it continues to reside within the body. It is embodied. Moreover, Kontos' approach is based on a foundationalist tradition that does not require social relations for the embodied self to be emergent, but allows us to "understand how culture and social practices elaborate and construct the human body through endless social relations based on reciprocity" (Kontos, 2003, p. 160).

In recognising Kitwood's approach, Kontos (2003) acknowledges that his work on personhood and dementia care was influential in refocusing dementia care away from a purely biomedical model to a model that embraced other important social and psychological dimensions. In other words, a refined approach with the aim of looking for more "human rather than medical solutions" (Kitwood, 1997, p. 2). By situating people in the social-cultural world, through the application of Bourdieu's (1977) concept of habitus, Kontos acknowledged the social discourse of Kitwood and others. In contrast to Kitwood's personhood and PCC, and in an attempt to address the above gaps, Kontos' approach to selfhood is embodied and is emergent and agential at all times and is not inextricably linked to the mind (Kontos, 2003, 2004, 2005; Kontos \& Naglie, 2007a, b). In grounding her approach of embodied selfhood within a framework of Merleau-Ponty's (2014) intentionality of the body and Bourdieu's (1977, 1990) habitus, Kontos is responding to the Cartesian dualism argument of other scholars in the field of dementia care.

Through the work of Kontos, this section theoretically challenges the epistemology and ontology of Cartesian philosophy within the context of dementia health and social care and dementia care practices, including PCC practices built upon the foundations of Kitwood and others. Kontos' central claim is that selfhood is a fundamental level of embodied existence that does not involve cognitive consciousness as it resides in the prereflective body. A life-force that continues to engage with the world despite the loss of memory, and emanating from the body's power of natural expression of bodily movement, gestures and sociability, and the spontaneous and improvised socio-cultural dimension of the pre-reflective body. 


\subsection{Summary of key theoretical influences}

Four key theoretical influences were revealed from the literature and provided a theoretical framework for this study: embodied selfhood, EL, multi-sensory enriched environment for cognitive rehabilitation and ethical and moral influences.

\subsubsection{Embodied selfhood (Kontos)}

The basis of Kontos' argument sets the foundation to theoretically challenge the epistemology and ontology of Cartesian philosophy within the context of dementia health and social care that is currently dominant in western healthcare systems and dementia care practices. Her central claim is that selfhood is a fundamental level of embodied existence that does not involved cognitive consciousness, as it resides in the pre-reflective body, a life-force that continues to engage with the world, despite the loss of memory. Selfhood emanates from two sources, the first being pre-reflective bodily expression through movement, gestures and sociability, and the second source being the socio-cultural dimension of the pre-reflective body (Kontos 2003, 2004). This analytic framework facilitated extraction of evidence of selfhood that is embodied, emergent, and intentionally agential at all times. A selfhood that is not inextricably linked to the mind.

\subsubsection{Errorless learning}

Implicit memory pathway is relatively spared in dementia. Errors made during learning are strengthened in implicit memory through the priming effect upon subsequent experience, thought, or action. Learning is more efficient if errors can be avoided during the learning process (Evans et al., 2004; Kihlstrom et al., 2007). The most beneficial technique of EL for PWD is self-generated EL which contains the following elements: teaching techniques aimed at reducing the number of errors during acquisition of learning, effortful learning, small customised steps, verbal instruction, use of vanishing cues (de Werd et al., 2013, 2015) and modelling to facilitate imitation through observation (Kihlstrom et al., 2007; van Tilborg et al., 2011). The principle and strategies of EL facilitated teaching PWD computer-assisted exergaming with Able-X.

\subsubsection{Multi-sensory enriched environment for cognitive rehabilitation}

The theory relating to the effects of cognitive stimulation is based on the notions of 'cerebral plasticity', which allows the brain to form new synaptic connections to take 
over functions of other parts of the brain that may be damaged (Hallett, 2001; King et al., 2010; Prince et al, 2014). Integral to this theory is "experience-dependent neural plasticity", which argues that environmental input can influence the generation of new brain connections (Gelfand et al., 2014, p. 55), a view supported by Hallett (2001), Hijmans et al. (2011), King et al. (2010) and Koshibu, (2016). Multi-sensory, enriched environments include computer-assisted exergaming environments (Khan et al., 2016). In this study, the principles of EL and brain activation rehabilitation (Yamaguchi et al., 2010) ensured the integrity of the therapeutic environment and safeguarded the ethical interest of doing no harm (Drury, Francis \& Chapman, 2007).

\subsubsection{Ethical and moral influences of Kitwood}

Kitwood (1997) presents three important philosophical discourses: transcendence, ethics and social psychology, that collectively determine that to exist and to be, is sacred, and that each person has absolute value, and is treated with deep respect, is socially located and has a recognised role. In my various roles, I strove to ensure that these important philosophical discourses were respected and implemented.

\subsection{Chapter summary}

This chapter has identified that the clinical research addressing computer-assisted interventions for PWD using Able-X is non-existent. There is a scarcity of clinical literature that focuses on Able-X and stroke rehabilitation, and other neurological conditions. The current literature does not provide in-depth knowledge about the effects of computer-assisted exergaming, utilising Able-X, for PWD.

Reviewing the literature for effective psychosocial interventions for PWD is limited by the plurality of terms used to describe cognitive oriented therapies, with poor description of interventions being a methodological limitation. Even so, there is evidence from a large number of observational studies that cognitive activity programmes may be beneficial for both brain structure and function. However, the evidence is limited and caution is advised. There is a growing volume of RCTs, control studies, and critical reviews that focus on non-pharmacological invention literature relating to PWD. The selection of literature critically reviewed, reveals evidence that indicates that some psychosocial and psychotherapeutic interventions may have similar outcomes as 
pharmacological interventions, particularly for cognitive stimulation therapy and cognitive rehabilitation, which includes the EL interventional strategies that have been shown to be particularly beneficial in the aspects of cognitive functioning and QOL for PWD. However, these beneficial aspects are currently unexplored in relation to computerassisted interventions for PWD using Able-X. Also unexplored is the value of facilitating Able-X within an 'enriched' environment that aims to reduce the risk of making 'errors' during the intervention process.

A sparser literature provides evidence that non-pharmacological intervention therapy is different in every situational case and results obtained are dependent on all present, including the researcher/therapist, suggesting that successful nonpharmacological intervention therapy has at its core how the therapist communicates with the PWD and not what approach is taken. This literature is closely aligned to the large volume of literature on personhood and person-centred-models of care (Kitwood, 1997) which are critically reviewed. Personhood is defined as "a standing or status bestowed upon one human being, by others, in the context of relationship and social being" (Kitwood, 1997, p. 8). In the provision of therapeutic interventions, the social relationship is established, making this literature not only relevant, but essential in terms of facilitating computer-assisted interventions for PWD using Able-X, in an environment that is 'enriched' and free of malignant social psychology (Kitwood, 1990, 1993b, 1997). Also, by understanding and incorporating the philosophical discourses of transcendence, ethics, and social psychology, the potential risk of doing more harm than good, as revealed in a sparse literature relating to reality orientation therapy, is managed.

The chapter concludes with an illumination of the epistemology of embodied selfhood (Kontos, 2003, 2004), which provides a theoretical base to challenge the Cartesian mind-body dualism epistemology of Descartes, and the symbolic interactionist stance taken by Kitwood. The work of Kontos provides a framework and lens through which to see, understand, interpret, and communicate the actions of PWD. The conceptual framework of embodied selfhood, along with the principles and strategies of EL, provide the theoretical framework for this thesis. Additional theoretical influences were multisensory enriched environments and the moral and ethical influences of Kitwood. 


\section{CHAPTER THREE: METHODOLOGY AND METHODS}

\subsection{Introduction}

When undertaking research, coherence between the research approach and the research problem is essential. The overall research approach is informed by the nature of the research problem, philosophical assumptions of the researcher, methodology, and research methods. Coherence in all these aspects ensures trustworthiness and rigour. Existing literature focuses on the utilisation of Able-X for stroke rehabilitation. There is no literature currently explaining the benefit and potentiality of the Able- $\mathrm{X}$ as a cognitive stimulation gaming therapy for people with dementia (PWD), therefore the link between computer-assisted exergaming, utilising Able-X and QOL in PWD is yet to be determined. Understanding this link requires an approach that allows for observation of exergaming behaviours and interactions with the exergaming technology. This type of inquiry requires a pragmatic approach.

This chapter critically examines the philosophical, ontological, epistemological, and methodological positioning of the study. I critically explore and justify my rationale for using social pragmatism, combined with interpretive epistemology, within a collective convergent parallel CS methodological approach. Following this overview, I present the CS question, purpose, and components of each case, followed by a critical discussion of strengths and limitations of CS design. As CS is both a methodology and a method, I have chosen to present them within the same chapter for coherency. In the second part, an in-depth critical description of the multiple methods used to collect, manage and analyse my research data is presented. During the first two cases, some modifications were made, including decisions about the data collection process, assessments measures utilised, and the gaming interface and programme, all of which are explained throughout the chapter. I then provide a critical and reflexive discussion on trustworthiness and rigour. Reflexivity is one aspect of rigour and I strive to make apparent throughout the chapter how my multiple positions influenced the study's methodological positioning and the research process of the fieldwork. The chapter concludes with an examination of some of the key ethical issues relevant to researching vulnerable populations. 


\subsection{Methodological considerations}

This study recognises that PWD and others who know them well have different views of the world and of the people within it and these views and perspectives can change over time. There are many constructed realities in the form of multiple mental constructions that are socially, experientially and contextually based, and dependent for their form and content on the individuals who hold them and on the groups to which those individuals belong (Creswell, 2014; Lincoln, 1992). For people with AD and other dementias, the process of creating mental constructions diminishes over the disease trajectory due to deficits in explicit anterograde episodic memory (Alzheimer's Association, 2013b; Clare et al., 2000; Evans et al., 2004), as does their ability to cognitively and verbally express themselves (Jokel \& Anderson, 2012; Jokel et al., 2010). However, through their embodied selves, they are perceptively present and engaged with the real world in which they are situated (Kontos, 2004), and where others are present as their proxy or surrogate voice (Jonas, Schiffczek, Lahmeyer, Muller \& Riepe, 2011; Sugarman et al., 2007).

\subsubsection{Ontology and epistemology}

Philosophical assumptions are a basic set of beliefs that guide the orientation and the actions of the researcher. Creswell (2014) refers to these beliefs as four worldviews: postpositivism, constructivism, pragmatism, and transformative (Table 2).

Table 2. Four worldviews

\begin{tabular}{|c|c|}
\hline Postpositivism & Constructivism \\
\hline $\begin{array}{l}\text { - Determination } \\
\text { - Reductionism } \\
\text { - Empirical observation and } \\
\text { - Theasurement } \\
\text { Theory verification }\end{array}$ & $\begin{array}{ll}\text { - } & \text { Understanding } \\
\text { - } & \text { Multiple participant meanings } \\
\text { - } & \text { Social and historical constructions } \\
\text { - } & \text { Theory generation }\end{array}$ \\
\hline Transformative & Pragmatism \\
\hline $\begin{array}{ll}\text { - } & \text { Political } \\
\text { - } & \text { Power and justice oriented } \\
\text { - } & \text { Collaborative } \\
\text { - } & \text { Change-oriented }\end{array}$ & $\begin{array}{ll}\text { - } & \text { Consequences of actions } \\
\text { - } & \text { Problem-centred } \\
\text { - } & \text { Pluralistic } \\
\text { - } & \text { Real-world practice oriented }\end{array}$ \\
\hline
\end{tabular}

This study has broadly adopted an ontological approach of social pragmatism (Creswell, 2014; Onwuegbuzie \& Leech, 2005). A philosophical approach that is conceptualised as being on a continuum of co-existence with purists and pragmatists lying 
on opposite ends, and situationalists lying somewhere in between (Onwuegbuzie \& Leech, 2005). Purists contend that different approaches cannot and should not be mixed, and situationalists uphold the mono-method approach, but see complementary value in both purist approaches (Onwuegbuzie \& Leech, 2005). However, pragmatists advocate the integration of both approaches within a single study, where the researcher focuses on addressing the research problem rather than the purity of the philosophical approach (Creswell, 2014; Miles \& Huberman, 1994; Onwuegbuzie \& Leech, 2005; Yin, 2014).

The justification for social pragmatism was determined, in part, by the comprehensive literature review, which identified the gap in knowledge between QOL and computer-assisted exergaming with Able-X for people with AD and other dementias. Although there are validated instruments available to measure QOL and other associated aspects such as depression, anxiety, and cognition, none of these in isolation or collectively would allow a detailed exploration of computer-assisted exergaming utilising Able-X by PWD in the real-world environment. Also, there is a paucity of knowledge about how the improvements found, utilising validated instruments, translate back into the lived environment, and this is an important theoretical concept in this study, and could not be addressed comprehensively through either a postpositive or a constructivist approach in isolation. Elements of both were therefore required for this study.

To advance through the purist philosophical lens of postpositivism, the researcher begins with a theory or hypothesis based on the ontological position that there is a reality (truth) out there and ultimately, through robust postpositivist inquiry methods, the truth will be found and the ability to predict and control the phenomenon will materialise (Creswell, 2014; Yin, 2014). The epistemological positioning of the postpositivist researcher is dualist and objectivist, in that the researcher and the subject are separate entities as the researcher tries to study subjects without influence or being influenced. Thus, values and biases are said to be prevented and findings are asserted to be true (Creswell, 2014; Lincoln, 1992). This methodological positioning is experimental and manipulative within tightly controlled environments and is summarised by time and context-free generalisations that are deterministic of the laws of cause and effect and facilitate the collection of data that supports or refutes the theory or hypothesis (Creswell, 2014; Lincoln, 1992). The postpositivist worldview is reductionist and is based on careful 
observation and measurement of ontological object reality and epistemological objective duality (Creswell, 2014; Lincoln, 1992).

Clearly, a quantitative, postpositivist philosophical lens alone would not appropriately address the study question: How does Able-X affect QOL, cognitive functioning and physical functioning in people $\geq 65$ years who are living with dementia, and residing in three different assisted care settings in New Zealand? However, utilisation of some of the methods and tools from this approach would serve to validate qualitative findings. Furthermore, a more expansive approach would enable the capture of the multiple complexities of social and behavioural dimensions in the natural environment (Onwuegbuzie \& Leech, 2005).

Alternative, more expansive approaches are qualitative research paradigms, the methods of choice for sociological inquiries that aim to explore and emphasize broad understanding and deep insight of a phenomenon in its own context (Creswell, 2014; Lincoln, 1992; Robson, 2002). The constructivist worldview, sometimes called social constructivism, interpretivism (Creswell, 2014; Polit, Beck, \& Hungler, 2001), or naturalistic inquiry (Lincoln, 1992; Polit et al., 2001), lends itself philosophically to qualitative research approaches. Contrasting with the one Truth ontology of postpositivist inquiry, the ontological assertion of qualitative constructivism is relativist (Lincoln, 1992; Polit et al., 2001). The epistemological position of constructivist theory is monistic interpretivism, juxtaposed to the dualistic objectivism found in postpositivist inquiry. The monist philosophical approach asserts that the researcher and participants are merged into a single entity. The experience of participants leads the researcher to explore, interpret, and describe expansive and complex realities that are created subjectively by the interaction of this dyad (Lincoln, 1992; Polit et al., 2001).

The methodological positioning for constructivists is again the antithesis of the postpositivist paradigm. Rather than a controlled, contrived, or manipulated environment, research is focused in the natural environment where people, live, play, work, seek health care services and support, and where constructions are elicited and refined through a process of iterative interactions between the dyad to generate reconstructions of previously held constructions, or multiple realities (Creswell, 2014; Lincoln, 1992). In contrast to reductionist, deterministic underpinnings of postpositivist inquiry, constructivism is 
expansionist, non-deterministic and facilitates the emergence of new realities or constructs within an iterative consensual framework. Qualitative constructivist worldview is a more appropriate approach to answer the research question. However, I believe a plural approach would add even more value to this inquiry.

Although pluralistic approaches are used in this study, it is weighted towards an interpretivist epistemology (Creswell, 2014). Furthermore, this approach facilitates the collection of a "richer and stronger array of evidence that cannot be accomplished by any single method alone" (Yin, 2014, p. 66). This philosophical approach recognises that individuals seek understanding and subjective meaning from within the world in which they live. Meanings that are varied lead the researcher to look for complexity of views to generate knowledge and make use of quantitative statistical analysis to validate qualitative findings (Urdan, 2010). Additionally, if the design allows, make use of quantitative evidence to test qualitative findings (Span, Hettinga, Vernooij-Dassen, Eefsting, \& Smits, 2013).

Researchers from the transformative worldview have been critical of both postpositivism and constructivism. The transformative philosophical lens arose during the 1980s and 1990s as an alternative paradigm that challenged the postpositivist assumptions applied to marginalised individuals when researching other issues, such as power and social injustice and discrimination and oppression (Creswell, 2014). Also challenged is the constructivist approach, which is deemed to be lacking in its contribution to the change agenda for disadvantaged and disempowered groups (Creswell, 2014). A transformative lens holds that research inquiry needs are intertwined with politics and the political change agendas. Initially, I considered the transformative paradigm as being a possible philosophical orientation for my research, however, being closely associated with the ethical discourse of human rights and social injustice, it potentially lends itself to polarisation of individuals rather than seeing human life as interdependent and interconnected (Creswell, 2014); the latter two points being important theoretical concepts in this study. People with AD and other dementias are vulnerable, and some argue that this group is already polarised (Dewing, 2008; Morhardt \& Spira, 2013; Nolan et al., 2006a) and stigmatised (Bamford et al., 2007; Connell et al., 2009; de Vries et al., 2010; Swaffer, 2014; Teel, 2004), and the focus should move away from PWD and a new lens that views them as interconnected and interdependent in social, relational models of 
existence should prevail (Dewing, 2008; Mitchell \& Agnelli, 2015; Morhardt \& Spira, 2013; Nolan et al., 2006b).

Moreover, in this study, PWD were recruited along with at least two others, the next of kin (NOK) and the lead registered nurse $(\mathrm{RN})$, and to a lesser degree healthcare assistants (HCA) and diversional therapists (DT), with whom they were interconnected and interdependent. Therefore, although the study required me to be cognisant of elements of the transformative approach, such as empowerment and collaboration, the broader political and change agenda paradigms, although important in dementia research and relatively under-explored (Kontos \& Martin, 2013), were not considered to be influential in the successful completion of this study. Moreover, I believed that my choice of research approach would demonstrate a contribution to the QOL change agenda for this vulnerable group, and for these reasons, the transformative approach was rejected.

Another criticism that spans all research approaches relates to the absence of explicit description of ontological and epistemological orientation; although the broad research methodological orientation is usually clear (Creswell, 2014; Lincoln, 1992). Lincoln states that, "methodology interacts profoundly with epistemology and, likewise, epistemology is in part determined by ontology" (p. 379), all of which should be made apparent to the reader. In this study, the ontological approach of social pragmatism is combined with interpretive epistemology.

\subsubsection{Methodology: Making a case for Case Study}

CS methodology is both, "the process of an inquiry and the product of that inquiry" (Stake, 2003, p. 136). An inquiry strategy is required to investigate a contemporary phenomenon (the case) within its real-life context, in which the boundaries between phenomenon and context are not clearly visible (Baxter \& Jack, 2008; Stake, 2003; Yin, 2014). CS is a methodology that supports deconstruction and reconstruction of certain phenomenon, viewed through a variety of theoretical lenses, allowing multiple aspects of the phenomenon, within the context, to be revealed and understood (Baxter \& Jack, 2008). It is primarily used in the social sciences, commonly in qualitative inquiry, but not exclusively (Yin, 2014). Cases can be single or multiple in scope (Yin, 2014). The CS 
approach has coherency with the ontology of social pragmatism and interpretive epistemology (Creswell, 2014; Stake, 2003; Yin, 2014).

Stake (2003) identifies three types of CS: 'intrinsic', which are undertaken to get a better understanding of a phenomenon; 'instrumental', which aim to provide insights into an issue through exploration; and 'collective', which extend instrumental to several cases, all of which are heuristic, rather than determinative. Creswell (2014) expands the description of CS when multiple methods are utilised to acknowledge that qualitative and quantitative data are combined or integrated. Following careful consideration of Stake (2003), Yin (2014) and Creswell's (2014) description of types of CS methodology, this study fits the design of a collective convergent parallel CS. Figure 4 summarises the design, including methods and analysis.

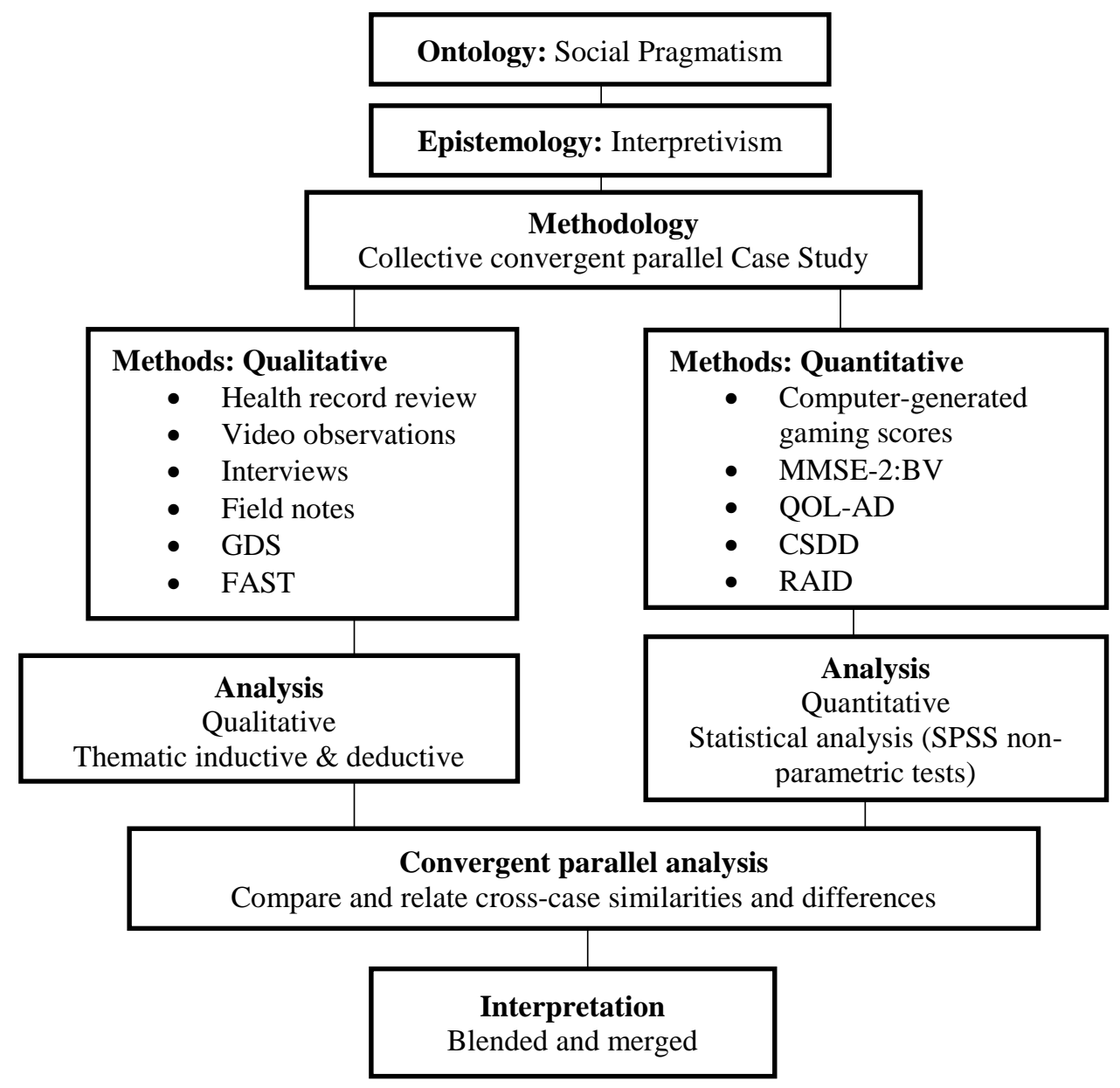

Figure 4. Study design 
In this design, qualitative and quantitative complementary data are generated and analysed separately before converging and merging to allow for comparisons and relationships to be comprehensively synthesised (Creswell, 2014; Yin, 2014).

The use of CS methodology in this research is guided primarily by the methodological approaches of Yin (2014) and Stake (2003). Yin (2014) provides a fivestep methodological protocol to guide researchers new to CS design. The first three steps of Yin's protocol were applied to construct the framework for my research, and the final two steps informed data analysis (see 3.3.5). The five steps are:

1. CS question

2. Purpose for exploratory studies, along with objective criteria (or propositions)

3. The components that contribute to a case

4. Logical linking of data to objective criteria/ propositions

5. Criteria for interpreting the findings

\subsubsection{Case study question, purpose and components}

\section{Research question}

How does Able-X affect QOL, cognitive functioning and physical functioning in people age $\geq 65$ who are living with dementia and residing in three different assisted care settings in New Zealand?

The CS question was formulated following a thorough review of the relevant literature determining gaps in knowledge, or what is yet to be known about the problem under inquiry. It was evident that the link between computer-assisted exergaming technology (Able-X) and QOL in PWD is yet to be made. However, a thoughtful and succinct question alone is not enough to bind the study direction. In exploratory studies such as this, a clear purpose and objective criteria were required to guide and direct the study (Stake, 2003; Yin, 2014). 


\section{Research purpose}

The purpose of this study was to explore if a programme of exergames utilising computer-assisted exergaming technology (Able-X), as an adjunct to existing activities and treatments, could deliver improvements in QOL and cognitive and physical functioning in people diagnosed with dementia.

\section{Research objective criteria}

1. To explore whether people age $\geq 65$ who are living with dementia, and are resident in care homes, achieve a:
a. QOL affect from using Able-X
b. Cognitive function affect from using Able-X
c. Physical function affect from using Able-X

2. To explore if Able-X, originally developed for stroke survivors, can be reconceptualised for people with dementia (to be reported in detail elsewhere).

Additionally, in CS research it is important to define the components of each case (Yin, 2014). In this study, each case comprises of the experiences of a PWD using the Able-X exergaming device, within the care home environment. Including:
a. Assessments (baseline and end-point)
b. Interview data (NOK, RN and significant others)
c. Video recordings of the person with dementia gaming with Able-X
d. Health record review
e. Field notes
f. Gaming scores produced by the Able-X
g. Bound by time

\subsubsection{Strengths and limitations}

One of the inherent strengths of CS methodology lies within its features of being both a comprehensive methodology and method of inquiry in which the researcher can explore in depth one or more individuals, events, activities or processes where there will be more variables of interest than units of analysis (Baxter \& Jack, 2008; Stake, 2003; Yin, 2014). CS benefits from the development of propositions or objective criteria and 
theoretical concepts to guide data collection and analysis, which all contribute to trustworthiness, reliability and congruence (Amerson, 2011; Yin, 2014). Cases are bound by time and activity, where researchers collect detailed information, using multiple data collection procedures over a sustained period of time in the field (Creswell, 2014; Simons, 2009; Stake, 2003; Yin, 2014). In this study, I spent seven months in the field, over a 10month period, excluding postscript interviews. Prolonged engagement in the field of research is one of Guba's (1981) criteria for demonstrating trustworthiness and credibility.

Another strength of CS research is triangulation, where multiple perceptions and a variety of procedures are utilised to clarify meaning and to identify the different ways phenomenon are perceived. Construct validity is established through the convergence of multiple sources of data to determine consistency of findings (Pharoo, 2006; Stake, 2003; Yin, 2014). Furthermore, triangulation is a validity strategy through which qualitative research methods can demonstrate credibility and confirmability (Guba, 1981; Kimchi, Polivka, \& Stevenson, 1991). In this study, three triangulation strategies were used: method, data, and analysis (Pharoo, 2006). Assessments of more than one point of difference were examined, and inferences made only when at least two unrelated but converged data sources correlated. For example, reports from proxy informants of improved hand-eye coordination were validated by improved gaming scores and improved functioning, and reports from proxy informants of improved BPSD were validated by improved depression and/or anxiety and/or QOL scores. Also, the study generated both qualitative and quantitative data (gaming scores and measures). Creswell (2014) argues that studies that collect both qualitative and quantitative data neutralises the weaknesses inherent in each data type. Amerson (2011) and Yin (2014) concur.

Conversely, the strength of having a rigid protocol or classification in qualitative research is challenged by Sandelowski (2010, p. 83), who advocates for a "living classification... built to contain within it the capacity to reconfigure the world", within a design. However, Sandelowski concurs with the need to analyse and interpret data, rather than celebrating its mere emergence, which has been the case in other methods such as qualitative description. Also, Sandelowski, agrees with the criticisms of Creswell (2014) and Lincoln (1992) regarding the lack or absence of an explicit description of ontological and epistemological orientation, and where findings are produced that show no evidence of, or congruence with the method claimed. 
Through the convergence of multiple sources of data, CS methodology can bridge the three main research approaches. However, this is also a limitation, due to the absence of an agreed language or clear description when constructing CS reports (Anthony \& Jack, 2009). Amerson (2011) considers this limitation is particularly widespread among nurses conducting CS. Overcoming this limitation requires familiarity with CS design. However, in the social sciences where it has long and established roots, confusion is less obvious (Yin, 2014).

The primary limitation of CS research is that it is relatively new as a research method, the bulk of studies being from the 1980s, however, the origins date back to the work of Campbell and Fisk in 1959 (Creswell, 2014). Anthony and Jack (2009) propose that CS is weaker or less rigorous than other methods. Lincoln (1992) concurs and argues that simple linear models, such as CS, generate simplistic, linear results. However, this view is challenged by Yin (2014, p. 3) who asserts that CS is "one of the most challenging of all social science endeavours", it is not only a linear process, it is also an iterative process that generates rich data rather than simplistic results. All case studies are unique; however, I agree with the view of Yin, and aim to demonstrate in this and subsequent chapters that this case study is iterative and will generate rich, comprehensive data.

\subsection{Methods}

\subsubsection{Recruitment strategy}

The approach of engagement and recruitment was based on the experience of my primary supervisor, Dr de Vries, an expert researcher in the field of dementia (Brooker et al., 2011; de Vries et al., 2010), an approach that required leadership of clinical managers in each care establishment in terms of identifying potential participants, obtaining consent of NOK and the lead RN and finally, the PWD. This multiple step recruitment approach was adopted to protect the interests of the PWD from coercion in the research process and to ensure that those who knew the PWD well, with whom they were interconnected and inter-related, were available to be recruited as a proxy voice of the PWD (Cacchione, 2011; Jonas et al., 2011; Sugarman et al., 2007). The approach is an ethically acceptable solution where consent and/or collateral information is required about another individual who is 
unable to provide the information for themselves (Health and Disability Commissioner, 1996; National Ethics Advisory Committee, 2012; New Zealand Medical Council, 2011).

Stage one of the process was to recruit the care establishments (Table 3). Sixteen care homes were identified through the regional dementia network and word of mouth. Each care home received a letter and information inviting them to participate in the study (Appendix 1) and an offer of a meeting to present the proposal and study requirements.

Table 3. Research time frame

\begin{tabular}{|c|c|c|c|}
\hline & Research activity & Commences & Completed \\
\hline 1 & $\begin{array}{l}\text { Stage one: } \\
\text { - Recruitment of care establishment } \\
\text { - Engage clinical leadership }\end{array}$ & November 2013 & April 2014 \\
\hline 2 & $\begin{array}{l}\text { Stage two: } \\
\text { - Recruitment of potential participants } \\
\text { - Initiate contact with NOKs and Lead RNs } \\
\text { - } \quad \text { Make contact with and obtain assent from PWD }\end{array}$ & November 2013 & July 2014 \\
\hline 3 & $\begin{array}{cl}\text { Site 1: } & \\
- & \text { Exergaming and data collection } \\
- & \text { Cases } 1 \text { and } 2\end{array}$ & November 2013 & April 2014 \\
\hline 2 & Study modification based on first two cases & May 2014 & June 2014 \\
\hline 4 & 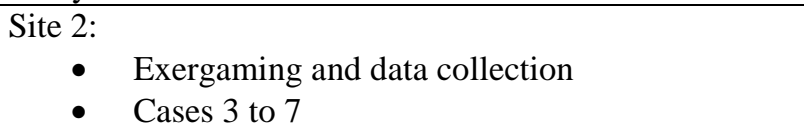 & July 2014 & August 2014 \\
\hline 5 & $\begin{array}{cl}\text { Site 3: } & \\
- & \text { Exergaming and data collection } \\
& -\quad \text { Cases } 8 \text { to } 10\end{array}$ & September 2013 & October 2013 \\
\hline 6 & Data analysis and synthesis & November 2013 & December 2015 \\
\hline 7 & Thesis and write up & July 2015 & December 2016 \\
\hline 8 & Dissemination & November 2014 & On going \\
\hline
\end{tabular}

Eight facilities responded, of which six expressed interest and two declined. The other eight failed to respond after two additional e-mail contacts. Following initial telephone contact, two interested sites were not available to participate within the research time frame due to other research commitments. Three of the remaining sites were recruited following completion of the access to unit agreement (Appendix 2), which included completion by a representative of the establishment of an on-line electronic signature required by the Ethics Committee, confirming that the site was suitable and that the signatory had read and understood the nature of and the requirements of the study. The three care homes were all in the North Island, one urban $\left(\right.$ Tenderlea $\left.^{9}\right)$, one suburban (Gardenside) and one rurally located (Lakelands). All provided residential and continued

\footnotetext{
${ }^{9}$ All names of establishments are pseudonyms.
} 
hospital level of care services, and Tenderlea and Lakelands provided additional secure dementia unit facilities. The fourth site was not recruited due to an objection to the use of the word 'dementia' in the paperwork, which is discussed in section 3.4.5.

Stage two of the recruitment process was two-fold, firstly to identify potential participants and secondly to initiate contact with the NOK. This role was delegated to the care home manager in liaison with the lead RNs, both of whom would know potential participants. Participants were initially identified utilising the inclusion and exclusion criteria (Table 4).

Table 4. Original inclusion and exclusion criteria

\begin{tabular}{|c|c|}
\hline Inclusion Criteria & Exclusion Criteria \\
\hline $\begin{array}{ll}\text { - } & \text { Age } \geq 65 \text { years } \\
\text { - } & \text { Diagnosis of dementia or showing } \\
\text { signs of memory impairment as } \\
\text { determined by care home management } \\
\text { and/or family members } \\
\text { - } \quad \text { Level of dementia mild to moderate as } \\
\text { measured by MMSE } \geq 10 \text { (Perneczky } \\
\text { et al., 2006) } \\
\text { - Resident in a care facility for at least } \\
\text { one year } \\
\text { - } \quad \text { Speaks English as first language } \\
\text { Ability to follow simple instructions in } \\
\text { English } \\
\text { Ability to physically hold the gaming } \\
\text { device and engage with computer } \\
\text { technology }\end{array}$ & 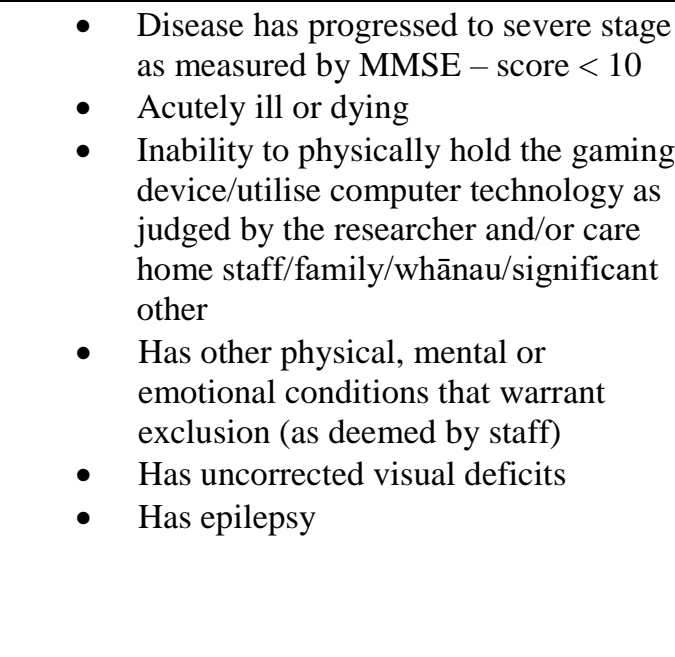 \\
\hline
\end{tabular}

However, the original criteria proved to be too restrictive, in that it became clear that the criterion MMSE $\geq 10$ was redundant because clinicians within each of the settings did not have access to current or previous cognitive screening assessment results. The clinicians called upon clinical judgment and a more global assessment, based on knowing the PWD well, to determine the level of dementia. Another challenge posed by the inclusion criteria was that four participants did not meet the length of residence criterion. Considering these challenges, a further review of the literature revealed that in NZ, up to 70\% of PWD are cared for in their own homes (Gee et al., 2001; Kirkman, 2011) and the majority of people with mild to moderate dementia do not reside in residential settings. For these reasons, the inclusion criteria were amended (Table 5). 
Table 5. Amended and final inclusion and exclusion criteria

\begin{tabular}{|c|c|}
\hline Inclusion Criteria & Exclusion Criteria \\
\hline $\begin{array}{l}\text { - } \\
\text { Age } \geq 62 \text { years } \\
\text { Diagnosis of dementia or showing } \\
\text { signs of memory impairment as } \\
\text { determined by care home management } \\
\text { and/or family members } \\
\text { - } \quad \text { Level of dementia is mild to moderate } \\
\text { as measured by MMSE } \geq 10 \\
\text { (Perneczky et al., 2006), or according } \\
\text { to clinical judgment } \\
\text { Resident in a care facility for } 2 \text { months } \\
\text { or more } \\
\text { - Speaks English as first language } \\
\text { Ability to follow simple instructions in } \\
\text { English } \\
\text { Ability to physically hold the gaming } \\
\text { device and engage with assistance with } \\
\text { computer technology }\end{array}$ & $\begin{array}{l}\text { - Acutely ill or dying } \\
\text { - Inability to physically hold the gaming } \\
\text { device/utilise computer technology as } \\
\text { judged by the researcher and/or care } \\
\text { home staff/family/whānau/significant } \\
\text { other } \\
\text { - Has uncorrected visual deficits } \\
\text { - Has epilepsy. }\end{array}$ \\
\hline
\end{tabular}

The second element of stage two recruitment process was to initiate contact with the NOK of each potential participant, as their contribution as proxy informants was a key aspect of this study, as was the proxy contribution of the lead RNs. This element of the recruitment process was delegated to the clinical managers at each location. This stage involved distributing pre-prepared study documentation, including an invitation to take part in the study (Appendix 3), information, and consent form (Appendix 4), an alternative, simplified information and consent form for PWD (Appendix 5), a proxy agreement allowing me to approach the PWD (Appendix 6), and information about the Able-X (Appendix 7). Once I was in receipt of all signed documentation I contacted the NOK and lead RN, to complete the consenting, permission giving and information sharing processes, following which, I made an approach to each participant to talk about the study and to obtain verbal assent (Black, Kass, Fogarty, \& Rabins, 2007; Black, Rabins, Sugarman, \& Karlawish, 2010; Cacchione, 2011; Cubit, 2010).

Of the 26 potential participants identified by the facilities, 12 engaged and 10 completed the programme. One of the participants who completed the programme was always accompanied by his wife, who was also a resident in the dementia unit, and although she was present and occasionally gamed, she was not a study participant as her NOK thought it would be too stressful for her, as would the separation from her husband (discussed in section 4.2.4). Two other participants did not complete the programme, one consistently disengaged from the gaming preferring to spend her time with me reading her 
simple books out aloud; she is not included in the study. A third participant who had been proxy consented by his NOK dissented on three consecutive occasions and therefore did not participate in the gaming experience (discussed in 3.4.2). Fourteen of those identified as potential participants did not advance to full engagement for several reasons, including family members $(n=4)$ not consenting as they thought the study would be too stressful, the person did not fit the study criteria as they had no documented evidence of dementia $(n=2)$, were acutely ill $(n=3)$, there was incomplete documentation $(n=3)$, or documentation arrived too late $(\mathrm{n}=2)$ for the person to be included.

The recruitment process was more difficult and time consuming than anticipated. I expected recruitment of the site, proxies and participants to take two to four weeks for each site, but it took six to eight weeks. Rather than one engagement visit, two or three were required. It was difficult to meet the manager and RNs during the same visit, and depending where the participants were located (residential, hospital or dementia unit) engagement visits had to fit in with staff availability which differed between settings and intra-setting. As with the recruitment of the establishments and RNs, recruitment and engagement strategies with NOK were also variable. Four NOKs wanted to meet at the establishment, one at their own place of residence, and five communicated all information by telephone.

\subsubsection{Exergaming with Able-X: The intervention}

The study provided an opportunity to utilise and explore existing NZ designed and commercialised computer-assisted exergaming technology (Able-X), developed specifically for stroke rehabilitation (Jordan, Sampson \& King, 2014; King et al., 2010; King, Hijmans, Sampson, Satherley, \& Hale, 2012; Sampson, Shau, \& King, 2012). The Able-X had two gaming options, the "Rehab Routine" (Im-Able Ltd., 2013, p. 17), a hands-off approach to gaming where the player completes the circuit challenge to get a baseline measurement of movement capability, which then automatically leads the player through challenges based on their personal movement capabilities as assessed by Circuit. This was the gaming option for the first two participants as recommended by my industry mentor. However, due to the predetermined gaming level and lack of familiarity with the exergaming technology, both participants had difficulty keeping focused for the game duration and making game selections. 
It is known that cognitive and emotional responses such as comfort, prediction, effortlessness, and speed are consequences of familiarity and vice versa (Son, Therrien, \& Whall, 2002). It will be apparent that the gaming outputs presented in the findings show that the first two cases played fewer games than the other eight participants. However, based on their experiences and opinions, I constructed a gaming sequence, selected from the second play option 'Rehab Challenges' (Im-Able Ltd., 2013, p. 17), that I believed would be more appropriate gaming therapy for PWD, as I was able to customise each challenge, including level of difficulty and length of play, I called the sequence the 'Cognitive Challenge', which resulted in improved engagement in the gaming programme (Figure 5).

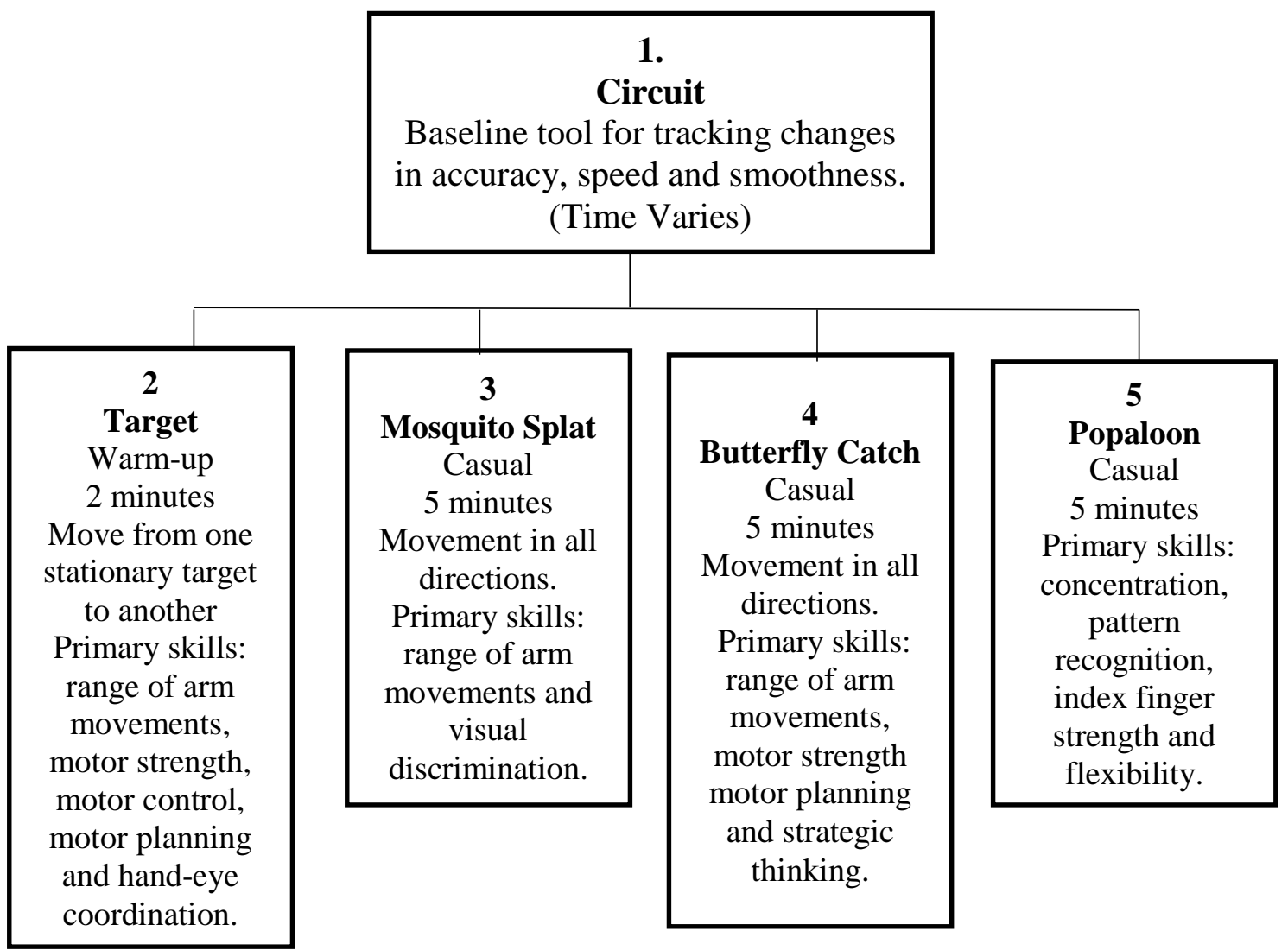

\section{Figure 5. The Cognitive Challenge}

The Cognitive Challenge consisted of one assessment challenge (Circuit) and four games (Target, Mosquito Splat, Butterfly Catch, and Popaloon). Circuit was designed primarily as a tool to assess accuracy, speed, and smoothness of movement and is based on a standard 'centre-out' assessment tool that has been used in neurological movement 
skill assessment for many years $\left(\operatorname{King}^{10} \&\right.$ Sampson ${ }^{11}$, personal communication $17^{\text {th }}$ April 2015). It was the intention of this study to look for 'intentional movement'. Target is the simplest of the Able-X suite of games, allowing players to move from one stationary target to another. One of the primary skills of this game is hand-eye co-ordination, the ability of the user to control his/her hands when presented with a visual challenge (Im-Able Ltd., 2013, p.25).

As players progress through the games, the selected games increased in difficulty and participants could build on the primary skill acquired from each preceding game (King \& Sampson, personal communication, April 17, 2015). Mosquito Splat and Butterfly catch require players to hit 'moving' targets, but avoid others. In addition to hand-eye coordination, two additional primary skills that these two games provide are concentration and visual discrimination. The final game, Popaloon, is the most complex challenge played by participants, as it required extra manipulation of the controller trigger and index finger. Popaloon also presents additional cognitive challenges of pattern recognition and concentration (Im-Able Ltd., 2013). Following on from the first two participants, the remaining eight participants played this gaming sequence, three times a week for four weeks (12 gaming sessions). Five of these played individually and three, from one site, occasionally played some gaming sessions in pairs or in small groups of three. This was purely a pragmatic solution for accessing participants from the secure dementia unit.

The gaming frequency was based on the study that had utilised the Able-X with stroke survivors (Hale et al., 2012; Hijmans et al., 2011). The exergaming intervention always took place in the morning, at a similar time for each participant. The exergaming sequence is 25 minutes; however, the exergaming process generated non-gaming moments that required time and attention such as self-reflection, reminiscence, validation, and relocation, extending the length of each session to approximately 45 - 60 minutes per person. Exergaming took place in an everyday environment such as an activities room. A summary of the frequency of exergaming, games played and group or solo gaming is shown in Table 33 (p. 199). Equipment used was a laptop computer, uploaded games, an

\footnotetext{
${ }^{10}$ King, from Medical Device Technology, Callaghan Innovation, Christchurch, NZ.

${ }^{11}$ Sampson, from Burwood Academy of Independent Living, Christchurch, NZ.
} 
extra computer screen, Able-X hand-held device, table, chairs, video recorder, tripod, and audio equipment.

\subsubsection{Data collection}

The first two cases provided an opportunity to test data collection tools and to determine which instruments would be used to measure change over time (Table 6). The data collection process commenced with the pre-gaming health record review (Appendix 8), which was repeated post-gaming to establish if change had been documented, and to facilitate data triangulation. The initial health record review took on average two hours; the follow up review took on average 15 minutes. The second stage of data collection was semi-structured interviews (Appendix 9). Interviews varied in length, but on average interviews took 17 minutes and 90 interviews were completed. All interviews were recorded and transcribed verbatim, resulting in 26 hours of audio transcription. All RN and participant interviews were completed on-site, as were four of the NOK informant interviews. One other NOK interview took place at a personal residence and five NOK were interviewed by telephone. To facilitate observation of exergaming and progress I used a video recorder as 'persistent observer', resulting in 62 hours of video recordings. No changes were made to either the health record review or the interview schedule templates during the course of the research.

The timing schedule for the exergaming intervention and associated interviews and assessments was underestimated. Initially I anticipated that six to eight weeks would be required for each site to complete the pre-and post-assessments, interviews and the gaming programme. However, this was a gross underestimation. It took 14 weeks at the first site. There were several extenuating circumstances including underestimation of the complexity of the recruitment and consenting processes, plus the testing and completion of extra measures. Two other delaying influences that were not factored into the timeline were acute illness and the emotional and physical effects of doing qualitative research. There was a viral outbreak of Norovirus within the residential unit, which resulted in the loss of four weeks. The impact of undertaking this study on me as researcher is discussed in section 3.3.6. 
Table 6. Data collection strategies

\begin{tabular}{|c|c|c|c|c|c|c|}
\hline & $\begin{array}{l}\text { Data collection } \\
\text { tools/strategies }\end{array}$ & $\begin{array}{l}\text { Baseline } \\
\text { measures } \\
\text { Pre-game } \\
\text { playing }\end{array}$ & $\begin{array}{l}\text { Game } \\
\text { Playing and } \\
\text { socialisation }\end{array}$ & $\begin{array}{l}\text { Mid-point } \\
\text { after } 6 \\
\text { gaming } \\
\text { sessions }\end{array}$ & $\begin{array}{l}\text { Game } \\
\text { Playing and } \\
\text { socialisation }\end{array}$ & $\begin{array}{l}\text { End-point } \\
\text { measures } \\
\text { after } 12 \\
\text { gaming } \\
\text { sessions }\end{array}$ \\
\hline \multicolumn{7}{|c|}{ QUALITATIVE } \\
\hline 1 & $\begin{array}{l}\text { Health record } \\
\text { review: } \\
\text { Demographic, } \\
\text { personal \& clinical } \\
\text { profile }\end{array}$ & $\checkmark$ & & & & $\checkmark$ \\
\hline 2 & $\begin{array}{l}\text { Semi-structured } \\
\text { interview (audio) } \\
\text { Proxy x } 2 \\
\text { Significant others }\end{array}$ & $\begin{array}{l}\checkmark \\
\checkmark \\
\end{array}$ & & $\checkmark$ & & $\begin{array}{l}\checkmark \\
\checkmark\end{array}$ \\
\hline 3 & $\begin{array}{l}\text { Video \& audio } \\
\text { recording of } \\
\text { participants } \\
\text { gaming \& } \\
\text { socialisation (12 } \\
\text { sessions) }\end{array}$ & & $\begin{array}{l}\checkmark \checkmark \checkmark \\
\checkmark \checkmark \checkmark\end{array}$ & & $\begin{array}{l}\checkmark \checkmark \checkmark \\
\checkmark \checkmark \checkmark\end{array}$ & \\
\hline 4 & $\begin{array}{l}\text { Field notes \& } \\
\text { observations }\end{array}$ & $\checkmark$ & $\checkmark$ & $\checkmark$ & $\checkmark$ & $\checkmark$ \\
\hline 5 & GDS/FAST & $\checkmark$ & & & & \\
\hline \multicolumn{7}{|c|}{ QUANTITATIVE } \\
\hline $6 a$ & $\begin{array}{l}\text { Mini-Mental State } \\
\text { Examination 2: } \\
\text { Brief version } \\
\text { (MMSE-2:BV) }\end{array}$ & $\checkmark$ & & & & $\checkmark$ \\
\hline $6 \mathrm{~b}$ & $\begin{array}{l}\text { Mini-Cog } \\
\text { (Cases } 1 \& 2 \text { only) }\end{array}$ & $\checkmark$ & & & & $\checkmark$ \\
\hline $7 \mathrm{a}$ & $\begin{array}{c}\text { Quality of Life AD } \\
\text { - } \quad \text { Participant } \\
\text { - } \quad \text { Proxy x } 2 \\
\end{array}$ & $\begin{array}{l}\checkmark \\
\checkmark\end{array}$ & & & & $\begin{array}{l}\checkmark \\
\checkmark \\
\end{array}$ \\
\hline $7 b$ & $\begin{array}{l}\text { DEMQOL } \\
-\quad \text { Participant } \\
\bullet \quad \text { Proxy x } 2 \\
\text { (Cases } 1 \& 2 \text { only) }\end{array}$ & $\begin{array}{l}\checkmark \\
\checkmark\end{array}$ & & & & $\begin{array}{l}\checkmark \\
\checkmark\end{array}$ \\
\hline 8 & $\begin{array}{l}\text { Cornell Scale for } \\
\text { Depression in } \\
\text { Dementia (CSDD) }\end{array}$ & $\checkmark$ & & & & $\checkmark$ \\
\hline 9 & $\begin{array}{l}\text { Rating for Anxiety } \\
\text { in Dementia } \\
\text { (RAID) }\end{array}$ & $\checkmark$ & & & & $\checkmark$ \\
\hline 10 & $\begin{array}{l}\text { Computer data: } \\
\text { Generated from } \\
\text { Able-X/prototype } \\
\text { \& computer games }\end{array}$ & & $\begin{array}{l}\checkmark \checkmark \checkmark \\
\checkmark \checkmark \checkmark\end{array}$ & & $\begin{array}{l}\checkmark \checkmark \checkmark \\
\checkmark \checkmark \checkmark\end{array}$ & \\
\hline
\end{tabular}

All outcome measures were selected based on their frequency of use in the literature with similar populations, and for their internal and external reliability, validity, 
sensitivity, and congruence with the design of this study and data collection strategies. One of the measures utilised with the first two participants was the Mini-Cog (Borson, Scanlan, Brush, Vitallano, \& Dokmak, 2000), a brief 3-minute cognitive screening measure that uses a three-item recall test for memory and a simple clock-drawing test, which serves as an informative distractor. This was selected as a potential alternative to the MMSE-2:BV (Folstein, Folstein, White, \& Messer, 2010), which had recently become subject to a charge by the current copyright holders (Psychological Assessment Resources). NICE-SCIE (2006/2015) and Thomas (2010) advised researchers to utilise alternative resources, the Min-Cog being one of them.

The MMSE-2:BV is a reliable and validated outcome measure. According to Folstein et al. (2010), it has internal consistency ( $\alpha^{12}$ 0.66-0.79), and test-re-test reliability ${ }^{13}$ coefficient that ranged from $0.44-0.86$ across all versions ( 0.44 for the MMSE2:BV). Several other validity tests were favourably reported, including interrater reliability of 0.94 to 0.99 utilising the one-way, single-measure intraclass correlation coefficients, of which there was $100 \%$ agreement for the items 1-16 on the MMSE-2:BV utilised in this study (Folstein et al., 2010). Conversely, problems with sensitivity of the instrument are reported (Lacy, Kaemmerer, \& Czipri, 2015), especially when looking for subtle cognitive changes in the healthy population (Schmand et al., 1995). However, the MMSE (Folstein et al., 1975) continues to be the most ubiquitous cognitive measure (Lacy et al., 2015) and for these reasons, the current version of MMSE-2:BV (Folstein et al., 2010), was the preferred cognitive measure for this study and was utilised to monitor change over time (baseline and end-point assessments). Application of the instrument was guided by the user's manual (Folstein et al., 2010). It has a total score of 16 points and measures registration, orientation to time, orientation to place, and recall. It comprises the first four sections of the traditional MMSE, now called MMSE-2:SV, which has a total score of 30 points.

The discourse of dementia includes classifications such as mild cognitive impairment, or mild, moderate, and severe dementia. To engage with these classifications

\footnotetext{
12 The Cronbach's alpha ranges from zero to one. A higher alpha level indicate a higher degree of item homogeneity (Folstein et al., 2010; Wells \& Wollack, 2003).

13 Test-retest reliability is concerned with repeatability of observations made on or by individuals. High reliability is considered to be 0.80 and above.
} 
however, there is a need to look beyond Folstein et al. $(1995,2010)$ who provide the score, relative to a normal population, of the same age and of a similar level of education, rather than staging of dementia as described above. In this study, I was initially interested in targeting people with mild to moderate dementia with a MMSE-2:BV score of $\geq 10$. However, prior to gaming commencement, but following completion of the consenting process, it became apparent that the first two participants were in the stage of severe dementia according to their MMSE-2:BV scores of 9 and 6 respectively when compared to generally accepted comparisons between MMSE scores and dementia staging, which according to Perneczky et al. (2006) is 26-29 dementia is questionable; 21-25 mild dementia; 11-20 moderate dementia; and 0-10 severe dementia. Similar stages are identified by Thomas (2010) and NICE-SCIE (2006/15). The lead RNs challenged the cognitive scores, arguing that based on clinical judgment and the judgment of their NOKs, both of whom knew the participants well, that these were suitable participants. Following discussion with my supervisors, I revisited the literature in search of a wider global assessment of functioning that would allow me to triangulate multiple clinical and assessment data sources that had already been collected for the first two participants, a technique that demonstrates the trustworthiness aspects of credibility and dependability in interpretive research methodologies (Guba, 1981).

I identified two scales that were pragmatic for this purpose: the Global Deterioration Scale (GDS) (Auer \& Reisberg, 1997) and the Functional Assessment Staging Test (FAST) (Auer \& Reisberg, 1997; Reisberg, 1988), both of which have been examined for reliability and validity, and are appropriate for global clinical assessment (Olde Rikkert et al., 2011; Oremus, Perrault, Demers, \& Wolfson, 2000). The GDS is a frequently used outcome measure from the global domain (Oremus et al., 2000), and describes seven distinguishable global stages from normality to severe dementia of AD type (Auer \& Reisberg 1997). The FAST describes a continuum of seven successive stages and sub-stages from normality to most severe dementia of AD type, all of which align with the GDS stages from where they were derived (Auer \& Reisberg, 1997; Reisberg, 1988). Both frameworks are brought together in a useful comparative matrix in Reisberg (2007, p.424) that plots useful data sources such as the MMSE scores, clinical diagnosis and the clinical dementia rating scale that I found particularly useful as an aide memoir, although I asked, I was not granted permission to reproduce it here. However, a cautionary note, the GDS/FAST matrix alludes to retrogenesis, a phrase coined by Reisberg, Kenowsky, 
Franssen, Auer, and Souren (1999) to describe the loss of mental abilities in old age in the opposite order in which they are acquired in childhood. Retrogenesis is a practice criticised by Kitwood (1990, 1993b, 1997) as a malignant social psychology that incorporates the risks of disempowerment, labelling, invalidation and objectification of PWD through infantilisation. This aspect of staging was not applied to participants in this study. The GDS and FAST staging were added to the battery of measures and used consistently in all cases other than one (case 5.2.8) where it was difficult to determine which aspects of disability were related to his multiple strokes, or to $\mathrm{VaD}$. The introduction of an extra assessment was reported to the Ethics Committee as part of the six-monthly reporting requirement.

Once I had completed the baseline data gathering and assessments, I had multiple sources of data that I was able to converge to stage each participant according to the GDS and FAST. This staging was more attuned to the clinical judgments of those who knew the participants well (RNs and NOKs). The participant with a MMSE-2:BV score of 9 was staged as moderate and the participant with a score of 6 was staged as moderately severe rather than severe as indicated by staging on the MMSE-2:BV score alone.

The second measure that was utilised in the first two cases but not for the remaining cases was the validated QOL measure the DEMQOL (Smith et al., 2005, 2007), which was used alongside the QOL-AD as a potential alternative, or additional measure of QOL. The DEMQOL is comprised of two slightly different scales, a 29-item, and a 31-item scale, that are completed during separate semi-structured interview with the PWD and the proxy informant respectively. Not only was the proxy questionnaire slightly longer, several of the items within the questionnaire differed from the PWD version, although each version is separated into three subsections covering feelings, memory, and everyday life.

The more global 13-item QOL-AD scale (Logsdon, 1996; Logsdon, Gibbons, McCurry \& Teri, 2002) contains similar items as the DEMQOL. It is also available in two scales, one for PWD, and one for a proxy (caregiver) informant. One of the criterion for utilisation of the QOL-AD is a MMSE score of $\geq 10$ for self-completion of the PWD option, otherwise researchers are advised to facilitate completion of the family member or caregiver version. In this study, it was important for me to hear the voices of the PWD in all aspects of this study so I decided to collect the assessment data from all participants 
and their proxy informants. Also, the QOL-AD scale was more time efficient and easier to complete than the DEMQOL. Selwood et al. (2005) found the same when comparing the QOL-AD with the DQoL, designed and tested by Brod et al. (1999).

Both versions have identical scale items, but the caregiver option has additional instructions for the rater. The QOL-AD measure had good internal consistency $(\alpha=0.88$ 0.89) and good test-re-test reliability for patient (0.76) and caregiver (0.92) reports (Logsdon et al., 2002). Moreover, validity was supported through the correlation of high QOL-AD scores and low levels of depression (Logsdon et al., 2002). The QOL-AD not only had a higher internal and test-re-test validity and reliability than the DEMQOL, it was more efficient to administer (15 minutes to complete both the PWD and the informant questionnaires), compared to the DEMQOL (40 minutes). Therefore, the QOL-AD was selected as being a pragmatic option for this study. It was utilised at baseline and endpoint from the PWD, NOK, and RN. The DEMQOL was only used for the first two participants, however, the data collected from this measure was not utilised during the data analysis process.

Associated and often linked with QOL in dementia is the absence of depression and anxiety, the latter being a potential symptom of the former. It is unclear from the literature whether depression itself is a causal factor or a symptom of dementia (Prince et al., 2014). Equally unclear is whether there is an association between anxiety and dementia. Anxiety is considered an important aspect of QOL for PWD as it is one of several indicators or symptom of depression (Alexopoulos, Abrams, Young, \& Shamoian, 1988) and therefore the absence of, or improvement in depression and anxiety scores is an important aspect of QOL.

Two measures were selected for measuring depression and anxiety in dementia: the Cornell Scale for Depression in Dementia (CSDD) (Alexopoulos et al., 1988) and the Rating Scale for Anxiety in Dementia (RAID) (Shankar, Walker, Frost, \& Orrell, 1999). The CSSD is a 19-item scale, rating signs and symptoms in the week prior to the assessment, and excluded symptoms arising from physical disability or illness. The total score is the sum of items $1-19$, and the range of scores is 0 to 38 . A total score of $\geq 8$ suggests significant depressive symptoms that required medical referral (Alexopoulos et al., 1988). The scale has high internal consistency ( $\alpha 0.84)$, good interrater reliability 
$\left(\mathrm{kappa}^{14}=0.63\right.$ for the severely demented) and (kappa $\left.=0.62\right)$ for the less severely demented (Alexopoulos et al., 1988). Further, this measure appears to be equally valid in non-demented populations (Kørner et al., 2006). The measure was completed as directed in the administration guidelines (Alexopoulos, 2002), through the interview process first with an informant $(\mathrm{RN})$, then with the PWD, following which the final result was decided and agreed upon by me in consultation with the lead RN. This measure was utilised to monitor change over time at baseline and end-point.

The RAID is an 18-item scale rating signs and symptoms within two weeks prior to the assessment, which excludes symptoms that arise due to physical illness or medication. The total score is sum of items 1-18, and the range of scores is 0-54. A score of $\geq 11$ is suggestive of significant clinical anxiety. The kappa values were moderate to good for test-retest reliability 0.53 to 1 and inter-rater reliability 0.51 to 1 , and the measure correlated significantly with other anxiety scales (Shankar et al., 1999). The scale was completed as directed in the administration guidelines, through the interview process first with an informant (RN), then with the PWD, following which confirmation of findings were sought through reviewing the clinical record for the same two-week period. The final result was decided and agreed upon by me in consultation with the lead RN. Table 7 provides a summary of data collection.

Table 7. Summary of data collection from all three sites

\begin{tabular}{|c|c|c|c|c|c|}
\hline Research activity & Subset & Site 1 & Site2 & Site3 & Total \\
\hline Days on site & & 17 & 21 & 18 & 56 \\
\hline \multirow[t]{3}{*}{ Number of Participants } & Primary & 2 & 5 & 3 & 10 \\
\hline & Proxy NOK & 2 & 5 & 3 & 10 \\
\hline & Proxy RN & 2 & 5 & 3 & 10 \\
\hline \multirow[t]{3}{*}{ Number of interviews } & Proxy NOKs & 6 & 15 & 9 & 30 \\
\hline & Proxy RNs & 6 & 15 & 9 & 30 \\
\hline & Postscript (RN) & 2 & 5 & 3 & 10 \\
\hline $\begin{array}{l}\text { Number of assessment with } \\
\text { primary participants }\end{array}$ & & 4 & 10 & 6 & 20 \\
\hline \multirow{2}{*}{$\begin{array}{l}\text { Number of assessments by } \\
\text { proxy informants }\end{array}$} & Proxy NOK & 4 & 10 & 6 & 20 \\
\hline & Proxy RN & 4 & 10 & 6 & 20 \\
\hline $\begin{array}{l}\text { Number of health record } \\
\text { reviews (pre \& post) }\end{array}$ & & 2 & 5 & 3 & 10 \\
\hline $\begin{array}{l}\text { Number of audio interview } \\
\text { hours }\end{array}$ & & $\begin{array}{l}9 \mathrm{hrs} \\
11 \mathrm{mins}\end{array}$ & $\begin{array}{l}10 \mathrm{hrs} \\
31 \mathrm{mins}\end{array}$ & $\begin{array}{l}5 \mathrm{hrs} \\
45 \mathrm{mins}\end{array}$ & $\begin{array}{l}25 \mathrm{hrs} \\
27 \mathrm{mins}\end{array}$ \\
\hline $\begin{array}{l}\text { Number of video gaming } \\
\text { observation hours }\end{array}$ & & $\begin{array}{l}7 \text { hrs } 28 \\
\text { mins }\end{array}$ & $\begin{array}{l}33 \text { hrs } \\
29 \text { mins }\end{array}$ & $\begin{array}{l}20 \mathrm{hrs} \\
26 \mathrm{mins}\end{array}$ & $\begin{array}{l}61 \mathrm{hrs} \\
23 \mathrm{mins} \\
\end{array}$ \\
\hline
\end{tabular}

\footnotetext{
${ }^{14}$ Kappa is a measure of this difference, standardized to lie on a -1 to 1 scale, where 1 is perfect agreement, 0 is exactly what would be expected by chance, and negative values indicate agreement less than chance (Viera \& Garrett, 2005, p. 360).
} 


\subsubsection{Data management}

Through robust data management and audit trail processes, trustworthiness in terms of confirmability and dependability can be demonstrated (Guba, 1981). CS research generates large volumes of data that requires management. Physical and electronic storage and security of data is an ethical requirement (National Ethics Advisory Committee, 2012). All data, including personal and organisation details gathered during this study were deidentified and stored securely (Table 8). Paper documents remain locked in filing cabinets in an office at the Graduate School of Nursing Midwifery and Health (Victoria University of Wellington). Electronic data are stored at a security level dependent on the level of deidentification, for example, no video and audio material is stored in the cloud-based system of Dropbox. Raw data (audio and video recordings) were only available to my academic supervisors and myself. The large volume of video data required extra storage space on the university secure server. Viewing of selected video imagery by industry representatives was required to inform product development of the Able-X. All viewing was in my presence and in a controlled and private environment. Industry representative and transcribers were required to sign a confidentiality agreement (Appendix 10).

Table 8. Secure storage and back up of electronic data

\begin{tabular}{|l|l|l|l|l|l|}
\hline Key files storage, security and back-up & A & B & C & D \\
\hline 1. & $\begin{array}{l}\text { PhD working files (Case data, data corpus sets, data analysis, cases, } \\
\text { thesis, field notes, literature searches etc.). } \\
\text { All de-identified. }\end{array}$ & $\checkmark$ & $\checkmark$ & $\checkmark$ & $\checkmark$ \\
\hline 2. & $\begin{array}{l}\text { PhD support files (Ethics, reports, pdfs, presentations etc.). All de- } \\
\text { identified }\end{array}$ & $\checkmark$ & $\checkmark$ & $\checkmark$ & $\checkmark$ \\
\hline 3. & End Note Library & & $\checkmark$ & $\checkmark$ & $\checkmark$ \\
\hline 4a. & Audio recordings & & $\checkmark$ & & $\checkmark$ \\
\hline 4b. & Audio transcripts & & $\checkmark$ & $\checkmark$ & $\checkmark$ \\
\hline 5a. & Video recordings & & $\checkmark$ & & $\checkmark$ \\
\hline 5b. & Video transcripts & & $\checkmark$ & $\checkmark$ & $\checkmark$ \\
\hline 6 & Gaming outputs & & $\checkmark$ & $\checkmark$ & $\checkmark$ \\
\hline STRATEGIES & & & \\
\hline A & Drop box account (1 TB) plus Packrat safety back up. & & \\
\hline B & External hard drive (2TB) - Password protected \& encrypted. & & \\
\hline C & C Drive laptop computer - Password protected \& encrypted. & & \\
\hline D & Extra storage (back up daily) Victoria University Server - Password protected \& encrypted. \\
\hline
\end{tabular}

\subsubsection{Data analysis and interpretation}

Researchers need to be overt and accurate in their description of how data were analysed, and ensure techniques used are congruent with the design of the study (Braun \& 
Clarke, 2006). Failure to identify the method of analysis affects the rigour and reliability of the work, making it difficult for others to evaluate and/or synthesise it with other similar studies and can impede research in related projects (Braun \& Clarke, 2006). CS research does not require a specified approach to data analysis (Robson, 2002; Yin 2014). Yin (2014) asserts that the process for analysing case studies is the least developed aspect of CS research; much depending on the researchers own style of rigorous reflexivity, empirical thinking, interpretation, and presentation of findings. Stake (2003) concurs, proposing that researchers conducting CS select one of two analytic strategies, categorical aggregation or direct interpretation, the latter when conducting intrinsic case studies and the former when the CS is of the exploratory instrumental type as in this study.

Thematic analysis (TA) is a form of categorical aggregation of data. An accessible approach that is flexible in that it does not stem from or is not tied to a particular theoretical or ontological position. The latter point is a limitation in that it could be said to be poorly branded in the sense that it does not exist as an agreed method of analysis, rather as an analytical strategy that is commonly utilised but often called something other than TA (Braun \& Clarke, 2006). TA is an iterative process through which patterns and themes are elicited, analysed, interpreted, and reported as a story that the researcher tells about the data in relation to the research questions (Vaismoradi, Turunen, \& Bondas, 2013). The emergent themes are a coherent integration of disparate data that constitute a finding that is important and related to the research question (Sandelowski \& Leeman, 2012), allowing the researcher to construct meanings and elicit new knowledge (Braun \& Clarke, 2006; Fereday \& Muir-Cochrane, 2006; Vaismoradi et al., 2013).

Due to the lack of an agreed method for conducting TA, I constructed a seven-step process (Figure 6) to ensure logical organisation and analysis of data that represents seven steps from raw data (data corpus) to extraction of data themes, based on the inductive and deductive tenets of TA. The process was informed by Braun and Clarke (2006) and Vaismoradi et al. (2013). 


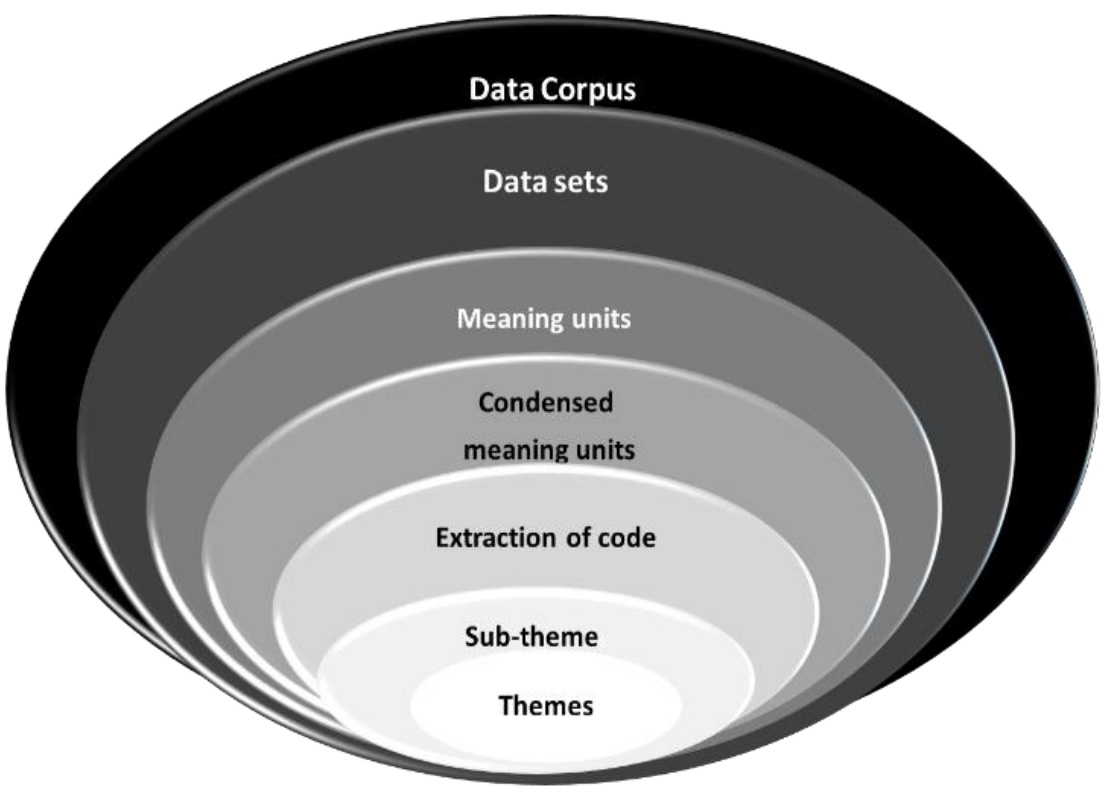

Figure 6. Seven steps from data corpus to theme

Braun and Clarke (2006) argue that researchers should be overt about their intent to generate either rich thematic description, where some depth and complexity is lost, or to generate in-depth detailed accounts of specific themes within the data. In this study, I strove to do both by utilising a hybrid process of data driven inductive approach of coding, complemented by priori codes derived from the objective criteria and theoretical frameworks which allowed what is yet to be known about exergaming to be integral to the process of deductive TA. Both approaches enable themes to be constructed from within the data (interview transcripts, field notes and video recordings). Every effort was made to ensure the subjective voice of participants, in their real world, was not replaced by an alternative over constructed non-existent reality, which is a risk associated with qualitative inquiries that lack rigour and trustworthiness (Fereday \& Muir-Cochrane, 2006). Figure 7 illustrates data sorting into logical segments (data-corpus sets) that best fit with each research objective criteria, exemplifying the first two stages of data organisation process, and step four of Yin's CS methodology protocol that requires logical linking of data to objective criteria. 


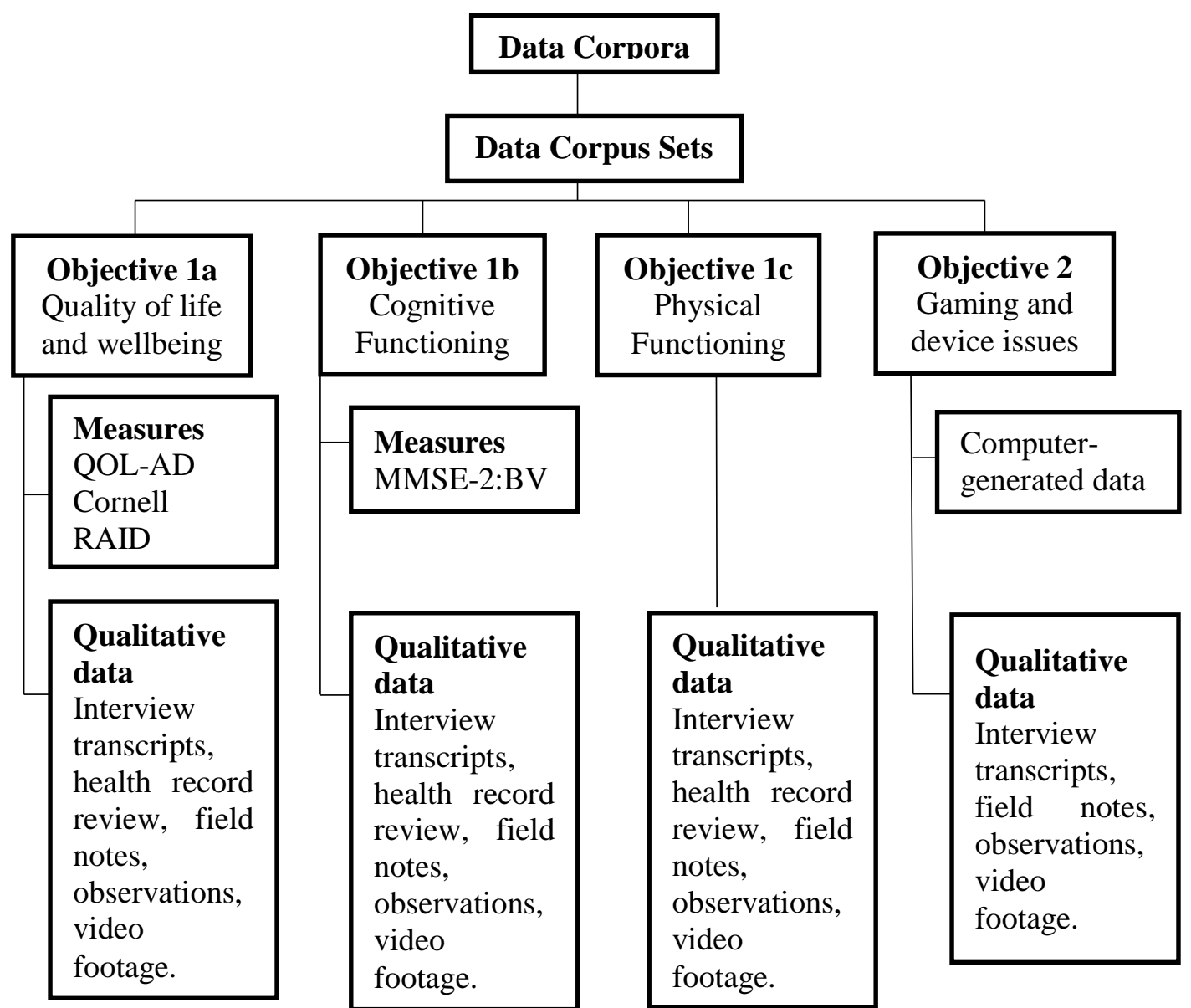

Figure 7. Data sorting: Logical data corpus sets

Stages 3-7 of the process required the data extraction of 'meaning units' (words, phrases, sentences or multiple text that conveys a significant meaning) which resulted from the iterative process of reading and re-reading each transcript multiple times. Stage 8 was a process of condensation to generate the 'main gist' of the meaning unit from which codes were identified, combined with codes derived from the objective criteria and theoretical frameworks which were used to analyse each data corpus set. Data were categorised into units of analysis, which were organised into themes. The data analysis process from meaning units to development of themes is demonstrated in Table 9, which is also the fifth and final stage of Yin's protocol for conducting CS research. 
Table 9. Example of raw data meaning unit to sub-theme of BPSD

\begin{tabular}{|c|c|c|c|c|}
\hline $\begin{array}{l}\text { Meaning Unit } \\
\text { (Reference in data } \\
\text { corpus set) } \\
\text { [Interpretation] }\end{array}$ & $\begin{array}{l}\text { Condensed } \\
\text { meaning unit- } \\
\text { [Interpretation] }\end{array}$ & Code (s) & $\begin{array}{l}\text { Sub-Theme/Unit } \\
\text { of analysis } \\
\text { [Interpretation] }\end{array}$ & $\begin{array}{l}\text { Theme } \\
\text { [Interpretation] }\end{array}$ \\
\hline $\begin{array}{l}\text { Joy: calmer and less } \\
\text { anxious and less pacing } \\
\text { Bill: His whole body } \\
\text { and mind appeared } \\
\text { more alert and aware } \\
\text { (VN:19/8:2676). } \\
\text { Iris: "she is not pacing, } \\
\text { agitated or looking to } \\
\text { escape" } \\
\text { (RN:I:28/7:2049). } \\
\text { Iris: "not wandering } \\
\text { about as much" } \\
\text { (RN:I:16/4:938). } \\
\text { Clara: alert and very } \\
\text { eloquently wakened, } \\
\text { (VN:28/7:) } \\
\text { Bill: "he's much more } \\
\text { relaxed...calmer } \\
\text { (RN:I:21/8:1714) } \\
\text { Iris: she was really } \\
\text { lively" } \\
\text { (RN:I:15/1:1326) } \\
\text { Alana: "was } \\
\text { depressed... [now] she } \\
\text { has a more positive } \\
\text { mood } \\
\text { (HCA:I:2/10:1049, } \\
\text { 1088) } \\
\text { Alana: her restlessness } \\
\text { had reduced (RAID) }\end{array}$ & $\begin{array}{l}\text { Behaviour is calm } \\
\text { and more relaxed, } \\
\text { although is bright, } \\
\text { interested, awake } \\
\text { and lively. }\end{array}$ & $\begin{array}{l}\text { Abscond/ } \\
\text { Anxiety } \\
\text { Alert } \\
\text { Awake } \\
\text { Aware } \\
\text { Calm } \\
\text { Depression } \\
\text { Escape } \\
\text { Lively } \\
\text { Mood } \\
\text { Pacing } \\
\text { Relaxed } \\
\text { Restlessness } \\
\text { Wandering }\end{array}$ & $\begin{array}{l}\text { Reduced } \\
\text { wandering and } \\
\text { pacing } \\
\text { Reduced } \\
\text { absconding } \\
\text { Improved } \\
\text { depression and } \\
\text { anxiety scores } \\
\text { More lucid and } \\
\text { wakeful }\end{array}$ & $\begin{array}{l}\text { Quality of life } \\
\text { improved through } \\
\text { reduced } \\
\text { behavioural and } \\
\text { psychological } \\
\text { symptoms of } \\
\text { dementia }\end{array}$ \\
\hline
\end{tabular}

Early real-time and iterative exploration of the data, using the reflexive techniques of inductive and deductive analysis and constructive synthesis, culminated in the revelation of important codes, patterns, themes and interrelationships (Braun \& Clarke, 2006; Fereday \& Muir-Cochrane, 2006; Stake, 2003; Vaismoradi et al., 2013; Yin, 2014). Where units of analysis fitted more than one thematic domain, decisions were made to present these findings where they fitted best. For example, it was impossible to improve gaming scores without controlled physical movement. This improvement, where quantifiable, was identified in controlled physical improvement, such as improved handeye coordination. Similarly, video data was analysed through multiple viewing of the video footage and TA procedures described previously. Gaming codes were identified and grouped together to create the first three themes 'Sentient interaction with the social gaming environment', 'Initiation and gaming advancement in partnership', and 'Levels of 
gaming intentionality', all of which focused on the gaming environment, including gaming initiation, engagement and progress. In the analysis of the video footage, six novel concepts were revealed: game shy, game enabled, game able, gaming by association, repeated success of easy gaming, and error starer-error inertia. These six novel concepts manifested inductively from the data and are may be generalisable to other situations.

Real-time evaluation of the data from the first two participants (video footage and field notes) revealed emerging recommendations for improvement of the Able-X for PWD, which was shared with representatives from Im-Able Ltd. ${ }^{15}$ and Callaghan Innovation in October 2014. Ongoing recommendations for improvement when they emerged were reported through meetings and the three-monthly reporting process ${ }^{16}$. All recommendations for industry will be reported elsewhere due to the word limitations of this thesis.

All utilised outcome measures (RAID, CSDD, MMSE-2:BV, RAID \& QOL-AD) generated categorical data at ordinal level. Ordinal data indicates qualitative differences in location on a scale and in what direction the difference is located (Urdan, 2010). In contrast variables generated by computed outputs from participant gaming scores, are continuous level data that indicate relative value and distance in terms of increasing or decreasing scores (Urdan, 2010). Data were analysed using Statistical Package for the Social Sciences Version 22 (IBM Corp., 2013), guided by Mehmet and Atkin (2003), Mehta and Patel (2012), Pallant (2011), Siegel (1956) and Urdan (2010), following which, all calculations were reviewed by a statistician. Where appropriate, descriptive and nonparametric statistics were applied to data that lent itself to this level of analysis. The threshold for $\mathrm{p}$ is $\alpha=0.05$.

Measures of central tendency were calculated, including median, interquartile range (IQR) and range for ordinal data, plus mean and standard deviation (SD) for continuous level data. Results confirm that the data was not normally distributed. Additional testing with the Shapiro-Wilks test of normality for small population (Mehmet

\footnotetext{
${ }^{15}$ Im-Able Ltd. Lower Hutt, Wellington, NZ.

${ }^{16}$ Agreed quarterly reporting as per template report to investmentsupport@ callaghaninnovation.govt.nz.
} 
\& Atkin, 2003) confirmed non-normal distribution of data. Statistical tests of choice are dependent on assumptions relating to the data, these assumptions are presented below.

The number of cases in this multiple CS was 10. The application of statistical test on data is dependent on assumptions regarding distribution of data, sample size, and level of data (Mehmet \& Atkin, 2003). Having established the data were non-normally distributed, small in number, at ordinal and continuous level, nonparametric statistical analysis by exact methods was selected. Exact tests are the preferred nonparametric tests for small, sparse, or unbalanced data, where the independent variable is measured at the ordinal or continuous level, and consists of two related groups, from which reliable inferences can be formulated (Mehta \& Patel, 2013). There is debate in the literature questioning the validity of application of large sample theory to small population data (Mehta \& Patel, 2012). Taking all these factors into consideration, data generated by this study was tested using the Wilcoxon signed-ranks test, a nonparametric exact test suitable for use on data that is small in number of ordinal or continuous level, including repeated observations of the same subject (Mehta \& Patel, 2012; Siegel, 1956). An alternative nonparametric test is the Sign test, however, the Wilcoxon signed-ranks test (exact test) is considered more powerful (Mehta \& Patel, 2012), thus justifying my choice of nonparametric data analysis.

In this collective CS, each case was written up separately to present an overview of the individualistic nature of each case, within the exergaming environment. In Chapter Four, each case is presented using a story-telling framework, with a combination of thick, rich description and in depth detailed accounts of specific themes relative to the case. Chapter Five presents the cross-case analysis to cultivate the under-mined knowledge (Khan \& VanWynsberghe, 2008), which consists of the emergent themes and novel concepts present across all cases. The theoretical framework of embodied selfhood (Kontos, 2004) and the principle and strategies of learning without errors (Terrace, 1963) facilitates cross-case interpretation of each of the themes.

\subsubsection{Trustworthiness and reflexivity}

Demonstrating trustworthiness is an important aspect of interpretive research. The most common framework for assessing trustworthiness are the four aspects coined by 
Guba (1981): credibility, dependability, confirmability and transferability, all of which have been woven, where appropriate, throughout this thesis, but are summarised here, drawing on the framework of Guba, whilst applying examples from this thesis.

\subsubsection{Credibility}

Credibility, the most important factor in establishing trustworthiness, is demonstrated through conducting research in a believable and credible manner (Houghton Casey, Shaw, \& Murphy, 2013), which includes explicit description of ontology, epistemology, methodology and methods (Creswell, 2014; Lincoln, 1992, Stake, 2003; Yin, 2014), all of which have been explored and described in this chapter. Other aspects of credibility are demonstrated through prolonged engagement in the field and persistent observation in an effort to gain a comprehensive understanding and appreciation of the phenomenon being studied (Enosh, \& Ben-Ari, 2016; Guba, 1981; Houghton et al., 2013). In this study, seven months over a 10-month period were spent in the field conducting research activities. Video was used as a data collection method, which is becoming increasingly popular in dementia research (Einang Alnes, Kirkevold, \& Skovdahl, 2011; Hammar, Emami, Götell, \& Engström, 2011; Hansebo \& Kihlgren, 2001; Kontos, Miller, Mitchell, \& Stirling-Twist, 2015; Ridder et al., 2009; Williams, \& Parker, 2012). Sixtytwo hours of persistent observation were generated.

Additionally, credibility is demonstrated through data triangulation (presented in 3.2.4), data saturation (see section 3.3.6.3), and member checking. In this study, 50 percent of CS transcripts were checked by NOK, who advised on changes required to protect identity, no other significant changes were requested. The selection of which cases were member checked was determined by a delay in postscript interviews for five participants where the lead RNs were unavailable for comment due to extended leave or resignation. However, I managed to locate these nurses, who each agreed to postscript interviews.

\subsubsection{Dependability, confirmability and reflexivity}

One of the key components of dependability and confirmability is through audit trail rigour (Fereday \& Muir-Cochrane, 2006; Guba, 1981; Houghton et al., 2013). Establishing an audit trail that details methodological and interpretive decisions and judgments of the researcher makes it possible for others to discern and evaluate data 
collection, analysis, and interpretation and although they may not agree with the researcher's interpretation, they should at least be able to understand how it was reached (Houghton et al., 2013). In this thesis, I have made every effort to critically describe each step of the research process, which is reflexively documented throughout the chapters of this thesis, and specifically this chapter in terms of methodological and interpretive decisions and judgments.

Reflexivity provides a process through which critical examination of how the researcher's beliefs, assumptions and interactions/relationship with participant during the course of the fieldwork influence the data and interpretation, therefore explicit reflexivity increasing the rigour of the research process (Enosh \& Ben-Ari, 2016; Guillemin, \& Gillam, 2004; Jootun, McGhee, \& Marland, 2009; Lincoln \& Guba, 2000). For example, during gaming I turned off the gaming sound to mask negative noises and encouraged participants to ignore negative on-screen messages in an effort to keep the gaming environment and activity positive and as errorless as possible. I also regularly recalibrated the Able-X controller, as participants were not able to do this task for themselves. There is no doubt that principles of EL and brain activation rehabilitation influenced my interaction during gaming with Able-X. Furthermore, when a researcher is also identified as a professional nurse, this latter role has the potential to influence the researcherparticipant interaction (Alley, Jackson, \& Shakya, 2015; Jack, 2008; Jootun et al., 2014). The use of reflexivity has had a pivotal role in articulating how my position as both a nurse and researcher, influenced the philosophical and methodological positioning of the study and the research process of fieldwork. These considerations have been addressed throughout the chapters of this thesis in order to provide transparency of process, findings and written representations.

In Chapter One, I describe reflexively how my professional interest in dementia care research evolved. In addition, reflexivity is documented throughout the thesis as I justify the decisions and choices made during the research process. Here I critically examine how I, as a researcher, have influenced this study. My role was tetrachotomous (Huber, 2003): researcher, teacher, game partner, and study subject. It is not uncommon for the researcher to have multiple roles (Denzin \& Lincoln, 2003; Dickson-Swift, James, Kippen, \& Liamputtong, 2006, 2007; Wray, Markovic, \& Manderson, 2007). In my role as researcher, I researched the area of computer-assisted gaming/exergaming and dementia 
literature and revealed the importance of procedural steps when providing therapy sessions to PWD (Clare et al., 2000; Clare \& Wilson, 2004; de Werd et al., 2013, 2015; Dou et al., 2006; Ferland et al., 2013; Jones \& Eayrs, 1992; Laffan et al., 2010; Metzler-Baddeley \& Snowden, 2005; Schmitz et al, 2014; Thivierge et al., 2008; Yamaguchi et al., 2010). Furthermore, I became aware that the negative feedback loops in the gaming system may be motivational for one population, such as stroke survivors (Jordan et al., 2014; King et al., 2012; Hale et al., 2012; Hijmans et al., 2011; King et al., 2010), but may do the opposite for a population such as PWD who cannot self-correct errors made when learning new things (Evans et al., 2004).

In my role as a teacher, I took advantage of a practice framework for activating cognition utilising the five principles of brain activation rehabilitation (Yamaguchi et al., 2010), through the construction of an interventional procedure for gaming to ensure the gaming intervention process was consistent, enjoyable and underpinned by ethical standards (Table 10).

\section{Table 10. Procedure for exergaming with Able-X-initiation and progress}

1. Introduce self and project. Ensure empathetic verbal/non-verbal communication between therapist and participants, as well as between participants

2. Obtain assent for gaming

3. Escort to and from gaming room

4. Ensure environment is comfortable (heat/light/ atmosphere etc.)

5. Explain, demonstrate and initiate gaming. Continue with verbal instructions as required. Watch for independent gaming agency and reduce/increase verbal support as required

6. Praise participants to enhance their motivation

7. Offer participants some social role that takes advantage of his/her remaining abilities

8. Errorless learning for pleasant atmosphere and to maintain participants dignity

9. Celebrate the completion of each game

10. Review scores and progress graphs if requested (choice)

Procedural Step 1 (along with the recruitment process discussed in section 3.3.1) embraces the rights of each participant to be treated with respect; to be free from discrimination, harassment, coercion, and exploitation; and to enjoy effective communication (Alzheimer's Association, 2011; Health and Disability Commissioner, 1996). Step 2 ensures the right of each participant to be fully informed and to give consent 
within their level of capability (Health and Disability Commissioner, 1996), and ensures that the ethical interest of doing no harm is observed (Drury, Francis \& Chapman, 2007). The specific ethical issues relating to consent of participants in this study are discussed in section 3.4.2 and 3.4.3. Steps 3-5 ensures that each participant is safely mobilised from one environment to another and that the environment is comfortable in terms of heat, lighting and atmosphere, and that the rules of engagement are made clear, all of which contribute to the enriched therapy and learning environment (Yamaguchi et al., 2010). Steps 6-10 provide positive regard and encourage motivation for participants to utilise their remaining abilities that were identified through data collection processes (interviews with proxy informants and health record review). For example, five participants had used a computer before and for them, input from me was minimal, compared to those who had never used a computer. Step 8 reiterates the importance of the EL principle and related strategies (Terrace, 1963, Yamaguchi et al., 2010), which is one of the key theoretical concepts in this study.

In my role of game partner, I took it upon myself to try to contemporaneously manage the negative messages and noises generated by the Able-X games, through verbal support, guidance and encouragement, in line with what is known about EL strategies and PWD (Clare et al., 2000; Clare \& Wilson, 2004; de Werd et al., 2013, 2015; Dou et al., 2006; Ferland et al.,2013; Jones \& Eayrs, 1992; Laffan et al., 2010; Metzler-Baddeley \& Snowden, 2005; Schmitz et al, 2014; Thivierge et al., 2008). Steps 4-8 were my strategies for redressing the negative loops that were inherent in the Able-X system, that I foresaw in the light of the literature, and pre-empted what aspects would need to be managed.

Once in the gaming environment, my role was more teacher and gaming partner and to a lesser degree, study subject in my own research. Steps 5-6 specifically relate to the gaming initiation and progress with individualised support that varied across the cases. Steps 6-10 contain motivational strategies all of which contributed to enrichment of the therapeutic environment (Astell, 2004; Enosh \& Ben-Ari, 2016; George \& Whitehouse, 2011; Khan et al., 2016; Oyedobe \&Parveen, 2016; Prince et al., 2014; Yamaguchi et al., 2010), which is captured on the video footage. The video recorder became my persistent observer, relieving me from observer role, to allow me to submerge myself in the realworld gaming experience, where the participant(s) and I merged into a single entity, through which I was able explore, interpret and describe expansive and complex realities 
of computer-assisted exergaming utilising Able-X with PWD. The aim was to explore and emphasize broad understanding and deep insight of the computer-assisted exergaming with Able-X phenomenon in the real-world context (Creswell, 2014; Enosh \& Ben-Ari, 2016; Lincoln, 1992; Polit et al., 2001; Robson, 2002).

According to Dickson-Swift et al. (2006, 2007) and Wray et al. (2007), selfsubmergence in the real world of research postures physical and emotional risks for both participants and the researcher. In this study, the participant's emotional and physical wellbeing were protected by the strategies presented in this section and sections 3.4.2 and 3.4.3. Additional strategies were required to ensure my own wellbeing whilst immersed in the field. For example, in some cases, the near constant verbal prompting and constant repetitive instructions became exhausting for me as gaming partner. To alleviate this risk, I limited the gaming time, used the pause gaming option and took opportunities to engage in empathetic verbal and non-verbal communication, strategies as advocated by Astell (2004, 2010) and Yamaguchi et al. (2010). I also made time to discuss and reflect on my fieldwork with my supervisors as advocated by Dickson-Swift et al. $(2006,2007)$ and Wray et al. (2007). The procedural steps and strategies presented above, along with the principle of EL and the analytic framework of embodied selfhood (Kontos, 2003, 2004), influenced me as a researcher in how I prepared myself to undertake this research, how I influenced the gaming environment and how I constructed my framework for data analysis. A context that requires researchers to be reflexive and responsive to participant's emotional well-being as well as their own.

\subsubsection{Transferability}

Transferability is demonstrated through the collection of thick descriptive data (Braun \& Clarke, 2006; Guba, 1981; Houghton et al., 2013). In this study, this data consisted of 90 audio-recorded interviews (40 with RNs, 30 with NOKs, 20 with participants), which were all transcribed verbatim, resulting in over 26 hours of thick description. In addition, 62 hours of video recordings of the participants exergaming and ten pre-and post-gaming health record reviews added to the thick, rich, descriptive data; a selection of which are reported in the case studies in Chapter Four and in the cross-case analysis in Chapter Five. 
Transferability and credibility are further demonstrated through both theoretical saturation and data saturation, the former being relevant for theory building research such as grounded theory (Walker, 2012), and the latter, being relevant to all qualitative research designs, but often under reported (Fusch \& Ness, 2015; Guest, Bunce \& Johnson, 2006; Morse, Lowery, \& Steury, 2014; Wray et al., 2007). Data saturation is reached when the ability to elicit new knowledge has been attained; when more coding and additional cases are unlikely to generate new information; when only repetitions are noted (Fusch \& Ness, 2015; Wray et al., 2007); when the researcher determines that the collection of additional data will not contribute to answering the research question (Morse et al., 2014), and when the study can be replicated (Guest et al., 2006). In this study, other than expanding the methods to include the GDS/FAST, the collective convergent parallel CS methodological design and associated methods, and the processes of data collection and analysis, facilitated data saturation within the study period. On reflection, I am aware I reached data saturation by case seven in terms of utilising the Able-X with PWD, as no new information or themes were found in the final three cases; just repetitive evidence of what had already been extracted and coded during the first seven cases. Guest et al. (2006) found similar levels of data saturation after as few as six interviews, and within the first 12 interviews. However, due to every study design being different, there is no "one-size-fits-all method to reach data saturation" (Fusch \& Ness, 2015, p. 1409).

The major ethical issues that arise in a study like this, including recruitment and consent of PWD and an argument for proxy consent, are presented (see 3.4.2 and 3.4.3). This information will be especially useful for any researcher intending to undertake research with PWD. Also transferable is the procedure for exergaming with Able-X (Table 10), which lends itself to the execution of other forms of psychosocial intervention therapy. Similarly, the six original concepts (game shy, game enabled, game able, gaming by association, repeated success of easy gaming, and error starer-error inertia) that were revealed and that are indicative of the level of embodiment in relation to exergaming could be applied to other activities in which PWD interact with the world. Examples demonstrating this transferability are presented on page 228 and 229. 


\subsection{Ethical processes}

In addition to the ethical principles and the rights of participants, discussed in section 3.3.6.2 the participants in this study were vulnerable, in that all had reduced capacity to consent. Guillemin and Gillam (2004) distinguish between procedural ethical processes and ethical issues relating to practice, and the importance of reflexivity as a strategy for balancing ethical tensions and "important ethical moments" (p. 277) that arise, especially in qualitative research.

\subsubsection{Ethics approval}

In NZ, there are widely accepted procedures for ensuring researchers are bound by ethical and moral practices and high standards of academic integrity when conducting research with people who may not be able to give informed consent, or lack the capacity to do so (Health and Disability Commissioner, 1996; National Ethics Advisory Committee, 2012; New Zealand Medical Council, 2011). Researchers are required to seek to balance the vulnerabilities that arise due to the person's diminished capacity with the injustice that would arise from their exclusion from the benefits of research.

Swaffer (2014) found that stigma and discrimination is compounded by low numbers of people with dementia taking part in research activities. However, research involving one or more participants who have restricted capability to make independent and informed decisions about their participation in a study, as with this study, are considered more than minimal risk and, in NZ, fall into the category of requiring a full review by the Health and Disability Ethics Committee of New Zealand (National Ethics Advisory Committee, 2012). An application for a full review of this study was submitted to the Central Health and Disability Ethics Committee NZ in July 2013, following which both my primary supervisor and myself attended an interview with the Ethics Committee to present the study and answer questions relating to it. Following this meeting, additional documentation and information was submitted, including contact details of named advisors for both Māori and Pacific peoples and an additional, simplified, information and consent form for PWD (Appendix 5). Ethical approval for this study was granted in November 2013 (Appendix 11). 


\subsubsection{Consenting vulnerable adults}

Caregivers' efforts are acknowledged for the potential to be the surrogate voice (Sugarman et al., 2007) or proxy voice (Jonas et al., 2011) should the day come that the person with dementia is unable to verbalise their desires and wishes for themselves. Researchers working with people with dementia are increasingly calling upon the proxies to engage and provide consent for people with dementia to take part in research activities (Cubit, 2010; Overton et al., 2013). Proxies are usually a spouse, caregiver, or other trusted individual who knows the person well (Health and Disability Commissioner, 1996; National Ethics Advisory Committee, 2012; New Zealand Medical Council, 2011). De Vries et al. (2013) and Overton et al. (2013) recommend caution based on the results of a survey about how proxies made their decisions finding that some proxies reported that they would be willing to override the person's wishes, or persuade or influence the person if they perceived it was in their best interests. Although proxy consent is accepted as a standard of academic integrity, and was the first step of the consenting process in this study, some argue that proxy consent is not sufficient to safeguard the interests of vulnerable populations and more rigorous efforts, including participant assent is required (Black et al., 2007; Black et al., 2010; Cacchione, 2011; Overton et al., 2013).

In one case, a proxy NOK consented his relative for the study without the PWD's permission. It was clear to me by the second meeting that this participant did not consent or assent to taking part and after agreeing to meet with me to talk about the study he dissented on three consecutive occasions. This case demonstrates the researcher's responsibility to treat each person with respect, in a relationship that is free from discrimination, harassment, coercion, exploitation and to ensure the right of each person to be fully informed and to give consent within their level of capability (Health and Disability Commissioner, 1996). In so doing this demonstrates the qualities of a "sympathetic other" (Goffman, 1963, p. 37), illustrating a desirable code for conducting research with vulnerable populations.

\subsubsection{Assent and dissent}

Black et al. (2010), Cacchione (2011) and Overton et al. (2013) advocate for additional effort over and above proxy consenting processes in the context of research involving decisional impaired adults to determine how best to operationalise the concepts 
of assent and dissent, further assuring academic integrity. Assent requires individuals to have minimal understanding to be able to make a choice regarding participation. Obtaining assent and respecting dissent are widely adopted safeguards when conducting dementia research. In this study, assent was sought at every contact and dissent was binding if it was constant after an effort to relieve concerns and/or distress. The assenting process embraced the rights of each participant to be treated with respect, to be free from discrimination, harassment, coercion and exploitation and to enjoy effective communication and to be fully informed within their level of capability as required by the Health and Disability Commissioner (1996), and in so doing, addresses the concerns of Overton et al. (2013) of undue persuasion, influence and the risk of overriding the PWD wishes.

\subsubsection{Proxy rater (advocate)}

Utilising proxy informants beyond the consenting process in research is increasingly common (Cubit, 2010; Jonas et al., 2011; Overton et al., 2013). For example, in this study, proxy informants completed several outcome measures on behalf of participants. Many validated outcome measures have questionnaires specifically for proxy raters, including the DEMQOL (Smith et al., 2005, 2007) and the QOL-AD (Logsdon, 1996). However, Jonas et al. (2011) found that proxy ratings can be modulated by informant's variables, for example, a recent study found female caregivers with a feeling of poor wellbeing were likely to be biased in their rating of others, and tended to overestimate functional disabilities and underestimate the QOL of patients, therefore limiting the usefulness of the data in such cases. Correspondingly, Moyle, Murfield, Griffiths and Venturato (2012) found similar results, when using the QOL-AD questionnaire with a similar population and concluded that level of impairment in ADLs had an inverse effect on ratings by proxy informants, especially care staff. Likewise, Winzelberg, Williams, Preisser, Zimmerman and Sloane (2005) concluded that ratings of QOL by healthcare assistants may be influenced by their attitudes to dementia and their confidence in meeting the care needs of PWD, and Spector and Orrell (2006) found that residential care staff rated QOL differently to PWD, and the difference was directly correlated to low staff hope. To reduce the inherent limitation of utilising proxy informants, ratings were obtained from at least two sources who knew the PWD well and 
from the PWD themselves, providing multiple sources of data on each aspect of the inquiry. A cautious, but pragmatic solution, as recommended by De Vries et al. (2013).

\subsubsection{Ubiquitous language of dementia}

Dementia is a word that is difficult for people to say and/or hear, especially when it is in relation to someone close to them, resulting in the use of ubiquitous language such as 'short-term memory problems' and 'memory problems of old age' (Bamford et al., 2004). Equally critical of veiled narrative surrounding dementia is Swaffer (2014), who argues that failure to name dementia results in continuance of stigmatisation of PWD and delay in service access and further compounds the difficulty of recruiting PWD and their caregivers in research activities. During the recruitment process, one site expressed an interest in taking part in the study on the condition that reference to dementia be removed from all documentation and secondly, that I, as the researcher, refrain from using any language relating to dementia or dementia sub-types during the research process. Both conditions were unacceptable for two reasons: firstly, all documentation had been approved by the Ethics Committee and secondly, I felt ethically and morally challenged at the request to avoid the language of dementia diagnosis, consequently, the site did not progress to engagement. The above example demonstrates how the 'veiled narrative' described by Swaffer (2014) continues in the present-day care, and the potential injustice that arise from excluding PWD from the benefits of research (National Ethics Advisory Committee, 2012).

In another site, one participant's NOK crossed out every reference to dementia on the information sheet and consent form, as she was worried that her mum would pick it up in the documentation when alone and without opportunity to discuss the content and therefore be repeatedly reminded that she had dementia. Although I felt ethically and morally challenged by this action I understood the reasoning. The NOK and I agreed that I was free to use whatever language necessary to consent and gain assent during in faceto-face meetings, which alleviated my concerns in this case. Following a discussion with my supervisors, I accepted the edited documentation and this participant (Clara) progressed to full engagement. 


\subsubsection{Te Tiriti o Waitangi}

Te Tiriti o Waitangi recognises the Māori people as tāngata whenua (indigenous people of NZ). In conducting this research, I was cognisant of the significance of the Treaty of Waitangi and the principles embodied within the Treaty: partnership, participation, and protection. People of all ethnicities were eligible to take part in this project and the nature of the project was supportive of all participants throughout their participation and at their discretion. Māori, Pacific and Asian peoples are less likely to be in the care home setting than European New Zealanders (Ministry of Health, 2013a), however, the nature of the consenting process developed enabled me to seek cultural advice and guidance if required. I had named advisors for both Māori and Pacific peoples' ethnic groups and access other cultural advisory services as required by the Ethics Committee of NZ.

\subsection{Chapter summary}

The philosophical and methodological underpinnings of this study were critically described in this chapter. Also explored were the strengths and weaknesses of this study, including the rigour aspects of study design credibility and dependability. Bounded and guided by this approach, I critically presented the process of recruitment of three establishments, 10 participants, and at least two additional informants (proxy participants) that knew each of the participant well. CS research is an iterative process, therefore generating rich data that facilitated the exploration and deep understanding into computerassisted exergaming with Able-X and PWD. I presented a step-by-step audit trail of the research process, to facilitate replication by others who may wish to extend this research, demonstrating the rigour aspects of credibility, dependability, confirmability and the potential for transferability. Triangulation is a key element of CS research where multiple perceptions and a variety of procedures are used to clarify meaning and to identify the different ways the phenomenon is being seen, and construct validity is established through iterative process and convergence of multiple sources of data, to determine consistency of findings. The rich thick data generated through interviews and observation were merged with the findings from the outcome measures to establish and demonstrate rigour aspects of credibility of findings and transferability of the findings across the cases, but there is no claim for transferability beyond this study cohort. 
Throughout the chapter, I provided critical and reflexive discussion on how my multiple roles may have influenced the research process. In addition, I highlighted key ethical obligations that needed to be considered when conducting research with vulnerable populations such as PWD. In the following two chapters, I present the findings of my research. In Chapter Four, I present the individual 10 case studies. 


\section{CHAPTER FOUR: PRESENTATION OF CASES}

\subsection{Introduction}

In this first of two findings chapters, I present the 10 case studies that describe and explore the computer-assisted exergaming experiences of 10 older PWD who completed a four-week programme of cognitive rehabilitation therapy utilising the Able-X computerassisted exergame system. The chapter begins with an explanation of the terms used to describe the proxy voices within each case and how each of the voices and the context in which these voices were documented can be recognised by the reader through a unique referencing system. Each CS begins with a personal and clinical profile that documents the unique dementia journey of each case and situates them in one of three assisted care settings. A summary of gaming engagement, progress and quantitative gaming outputs are presented, followed by the individual case scores from baseline and end-point assessments for MMSE-2:BV, RAID, CSDD, and QOL-AD. Each CS concludes with a summary of the QOL findings unique to each case and a postscript statement indicating if the QOL findings were maintained at three, six, nine or 12 months post gaming.

\subsection{Cases}

Incorporated into each case are the proxy assessments and voices of those closest to each participant, including the next of kin (NOK), registered nurse (RN) and diversional therapist (DT). In some cases, voices of additional proxy agents such as the clinical manager (M) and health care assistant (HCA) are incorporated. Pseudonyms are used for participants and the residential establishments, and title abbreviations for all other informants are used to ensure identity protection. Voices can be recognised in the text through the detailed way in which they are referenced, for example (J:I:2/1:90) informs the reader that Joy $(\mathrm{J})$, was interviewed $(\mathrm{I})$, on $2^{\text {nd }}$ January $(2 / 1)$ and the raw data can be located on line 90 of the case master transcript. All data references follow the same pattern of date and line number, however prefixes change. Where appropriate the personal identifier is omitted if the voice of the narrator is obvious in the text for example, the NOK said "xxx" (I:7/2:502). Also, two participants have the same initials, but they are bounded by the case story. Some references relate to observational transcription notes such as: research field notes $(\mathrm{FN})$, video observation transcription notes (VN) and where live video footage (VL) can be found in the 62 hours of footage, if required, to evidence the claims 
made in the thesis. As I am the custodian of the data (including the live video footage), viewing of videos is only available to my thesis supervisors and my thesis examiners, the latter as part of the examination only.

\subsubsection{Joy $^{17}$}

When I look at other people, I'm glad I'm me....Even the mirror doesn't put me down....I still have a little joie de vivre. (J:I:22/1:96)

\section{Personal and clinical profile}

Joy was a small, slight nonagenarian female. She described herself as "an elderly person who doesn't think that she is" (VN:8/4:1695). In 2009, at the age of 86, she inadvertently drank dish-wash solution thinking it was wine. Family members became worried about her safety and ability to look after herself, and following assessments by her general practitioner and a psychogeriatrician, she was diagnosed with AD. In August 2012 , at the age of 89 , she was no longer coping safely at home, resulting in an urgent admission into a residential care bed at Tenderlea, located some distance from her home, friends, neighbours and neighbourhood, but closer to her daughter who was Joy's Enduring Power of Attorney (EPA) for Personal Care and Welfare. Joy was very settled at Tenderlea:

Ifeel pretty safe in here. I can't tell if I had been in my own home that I would feel the same. Here...they do things at the same time. Also, having my daughter. If I didn't have her here and near, I might feel different. (J:I:14/4:243)

In January 2014, Joy enjoyed excellent ambulation and independent mobility both within and outside the residential setting. She could manage all the basic ADLs, however IADLs were more of a challenge for her. For example, she had recently forgotten how to turn on her music centre, so could no longer access her beloved music. According to the Functional Assessment Staging Test (FAST) (Reisberg, 1988, 2007) and Global Deterioration Scale (GDS) (Auer \& Reisberg, 1997), Joy functioned within the boundaries of Stage 5. The stage where "individuals can no longer survive if left on their own" (Auer $\&$ Reisberg, 1997, p. 168). Stage 5 equates with a clinical diagnosis of moderate dementia

\footnotetext{
${ }^{17}$ The pseudonym Joy means "Jubilation" http://www.thenamemeaning.com/joy/
} 
(Reisberg, 2007). Other than taking regular amitriptyline for depression, no other moodaltering medications were administered during the gaming period.

\section{Engagement in gaming}

Joy needed full assistance to engage with the computer. Once gaming was initiated, she played with independent agency, assisted by moderate verbal prompting every 30-60 seconds. Joy had no day-to-day recollection of how to play the games but could ask questions to assist with gaming progress. Gaming with Joy was constantly punctuated by reminiscences or conversations that were triggered through the gaming experience. She played 10 of 12 solo gaming sessions over a 10 -week period ${ }^{18}$, declining on two occasions, to accommodate unexpected visitors. She played: Circuit $(n=10)$, Target $(n=7)$, Mosquito Splat $(n=6)$, Butterfly Catch $(n=1)$ and Popaloon $(n=3)$. Prior to gaming, she had never used a computer. Gaming sessions varied in length depending on Joy's motivation, enjoyment, and wellbeing. Every play session was as if she was playing for the first time, as she could not recall playing previously. After six sessions, her NOK reported, "she's enjoying it and she hasn't said anything negative. She's happy to do it to help you with your degree" (I:7/2:502).

On occasions, Joy became disengaged from gaming for one of three reasons: her constant battle with claustrophobia, the drift associated with the controller, and the impact of gaming on-screen messages, ${ }^{19}$ and noises ${ }^{20}$. Concerns about being in the confines of the gaming room caused physiological effects such as flushing, sweating, and shortness of breath, resulting in her desire to leave the room or abandon gaming. This reaction was not unexpected, as the RN, NOK, and Joy herself had alluded to her fear of closed spaces. I used several measures to manage this situation, for example, she was empowered to manage the environment (windows, door, space), a strategy proposed by Yamaguchi et al. (2010). Having a degree of control helped to an extent, other than on two occasions when gaming was discontinued and replaced with a visit to the garden and a rendition of one of her repetitive stories: "I have claustrophobia...it started after I found myself locked in

\footnotetext{
18 The gaming period was extended for the first two participants to accommodate testing of measures and game selection.

${ }^{19}$ Some on-screen messages were negative (too slow, try again). They interrupted Joy's gaming and caused gaming inertia.

${ }^{20}$ Some gaming noises were also associated with gaming errors and Joy thought she was doing something wrong and would stop gaming.
} 
wardrobe...I called out and eventually a neighbour heard and opened the door. Thank you for reminding me of the worst day of my life" (I:2/1:90).

Joy found the drift associated with the Able-X controller distracting and complained, "silly thing is we are not pointing at it [the target on the computer screen]" (VN:2/4:1504). I often needed to re-centre the controller as Joy could not remember how to do it herself and she found the intrusion irritating (VN:21/1/1629). By the midpoint, she had learned to ignore the drift and adapted her play by focusing on the screen and not the controller (VL:17/2:AA000806:00:00). The third trigger for disengagement, and the most disempowering, was the paucity of on-screen instructions and various negative messages, scores and sounds. For example, on the Circuit assessment challenge, other than the initial instruction, which disappeared from the screen too quickly, there were no on-screen prompts to encourage gaming action. The Target challenge provided more prompts both positive (excellent, good, well done) and some negative (too slow, try again, missed, try again). Mosquito Splat and Butterfly Catch games communicated positive and negative messages through noise and minus scores rather than on-screen words. These negative messages would trigger a response such as "It's not doing anything for me. I can't imagine why people would want to play these for hours on end" (FN:17/2:268,273).

\section{Gaming outputs}

Joy's gaming ability improved despite her being unable to remember previous gaming sessions and her variable gaming motivation. A comparison of baseline and endpoint Circuit graph and scores, demonstrates improved hand-eye coordination in terms of accuracy, smoothness and speed of movement. The biggest improvement was in gaming speed, 21 points. Joy also achieved improved scores for smoothness of movement (17 points) and accuracy of movement (14 points). Improvement was also evident on the Target challenge heat maps, where red indicates heat (activity) on the playing screen. At the baseline assessment, Joy was hitting targets mainly around the centre screen, but by end-point, she was seeing and reaching peripheral targets. Her end-point score for this game improved by 2000, evincing improvement in hand-eye coordination (Im-Able Ltd., 2013) (Figure 8). 


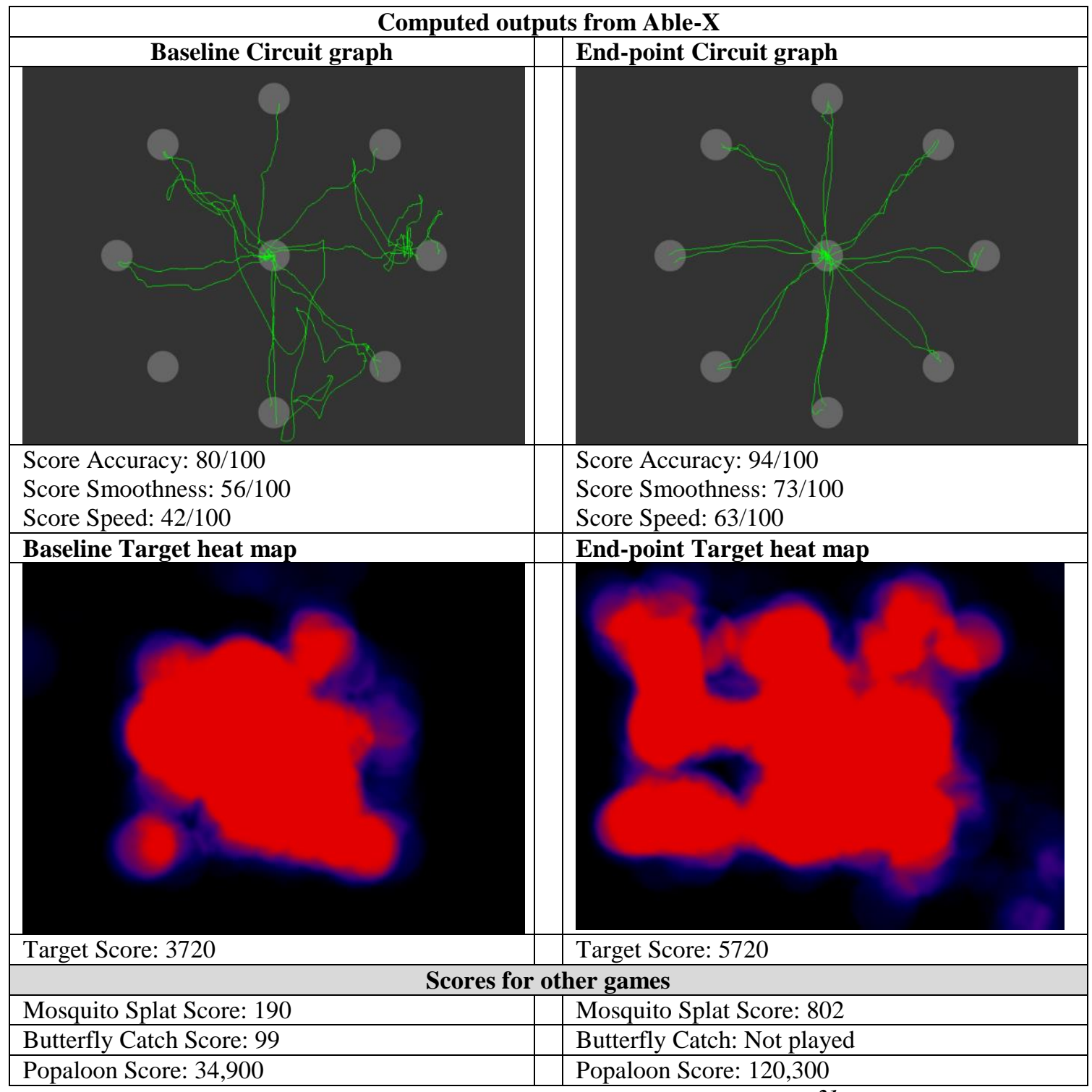

Figure 8. Joy: Computer-generated exergaming outputs ${ }^{21}$

Joy did less well on the Mosquito Splat and Butterfly Catch games, which build on the primary skills of hand-eye coordination and introduce the challenges of visual discrimination. Mosquito Catch was played six times with an improved score of 612 points. Joy only played Butterfly Catch once so there was no end-point score. She found some of the on-screen graphics in both games unpleasant, especially when they grew and crowded the screen, saying on two occasions "It's like being in my worst nightmare" (FN:17/2:268,273), following which the games were abandoned.

${ }^{21}$ Scores start at 0 at the beginning of each new game. 
Joy's biggest improvement was on the most difficult game in the sequence, Popaloon, which introduces extra manipulation of the Able-X controller and introduces two cognitive challenges of pattern recognition and concentration (Im-Able Ltd., p. 26). Although she only played Popaloon three times, her improvement was a huge score of 85,400 points, inferring improvement in cognitive ability of pattern recognition.

\section{Comparing baseline measures and end-point measures}

Joy's cognitive MMSE 2-BV improved by four points to 13/16 at the end-point assessment (Table 11). The extra points were gained through improved orientation to time ( 3 points) and place (1 point). Evidence of changed cognition was also provided by others, "[she is] less confused...it's amazing the change in her" (M:I:17/4:1235 \& 1258), and "she's been remembering your name which is really rare as she doesn't know the names of the staff" (NOK:I:7/2:474). Furthermore, improvement in cognitive ability of pattern recognition was demonstrated consistently as evidenced by the improved scores for the game Popaloon. However, there is no claim of improved concentration, as Joy required moderate verbal prompting.

Table 11. Joy: Summary of baseline and end-point measures

\begin{tabular}{|l|r|r|}
\hline Validated measure & Baseline & End-point \\
\hline MMSE 2-BV & $9 / 16$ & $13 / 16$ \\
\hline RAID & $6 / 18$ & $5 / 18$ \\
\hline CSDD & $2 / 38$ & $2 / 38$ \\
\hline QOL-AD: Self-report & $43 / 52$ & $40 / 52$ \\
\hline QOL-AD: RN Proxy & $47 / 52$ & $47 / 52$ \\
\hline QOL-AD: NOK Proxy & $34 / 52$ & $38 / 52$ \\
\hline
\end{tabular}

The slight reduction in anxiety RAID score by one point, infers a slight but positive change in anxiety levels, further affirmation of this was evident: "I think she is much calmer and less anxious" (M:I:17/4:1219); "I don't see her as stressed as much or as anxious" (DT:I:17/4:1102); "She's a lot calmer, she's not complaining about things" (M:I:17/4:1211). Joy's very low depression score of 2/38 was unchanged, however, improvements were noticed. The NOK reported that she is "certainly more positive" (I:24/4:622). The Manager reported positive changes more aligned to BPSD: 
There is a very clear difference that you can see, she's not pacing, she would usually be pacing...up and down the stairs....She's doing less and less of outside wandering and her inside wandering and mobility is good and purposeful...[she] passes and chats to...staff and goes to courtyard. (I:17/4:1268, 1210)

In terms of the Quality of Life-Alzheimer's Disease Scale (QOL-AD), there was one point improvement over the course of the study across all ratings. Joy's NOK rated four points improvement overall, however, the $\mathrm{RN}$ rated the total score unchanged. Joy perceived a reduced overall score of minus three QOL points (Table 12).

Table 12. Joy: Quality of life points change pre-and post-gaming

\begin{tabular}{|c|c|c|c|c|c|c|}
\hline Items on the QOL-AD questionnaire & $\begin{array}{l}\text { Self } \\
\text { B }^{*}\end{array}$ & $\begin{array}{l}\text { Self } \\
A^{* * *}\end{array}$ & $\begin{array}{l}\mathbf{R N} \\
\text { B }^{*}\end{array}$ & $\begin{array}{l}\mathbf{R N} \\
\mathbf{A}^{* *}\end{array}$ & $\begin{array}{l}\text { NOK } \\
\text { B }^{*}\end{array}$ & $\begin{array}{l}\text { NOK } \\
\mathbf{A}^{* *}\end{array}$ \\
\hline Physical health & 3 & 3 & 3 & 3 & 3 & 4 \\
\hline Energy & 3 & 3 & 4 & 3 & 2 & 2 \\
\hline Mood & 3 & 3 & 4 & 4 & 3 & 3 \\
\hline Living situation & 4 & 3 & 4 & 4 & 3 & 3 \\
\hline Memory & 3 & 3 & 2 & 2 & 2 & 2 \\
\hline Family & 4 & 3 & 4 & 4 & 4 & 4 \\
\hline Marriage (closest relationship) & 4 & 4 & 4 & 4 & 3 & 4 \\
\hline Friends & 3 & 3 & 3 & 4 & 3 & 2 \\
\hline Self as a whole & 3 & 3 & 4 & 3 & 2 & 3 \\
\hline Ability to do chores & 3 & 3 & 3 & 4 & 2 & 3 \\
\hline Ability to do things for fun & 3 & 3 & 4 & 4 & 2 & 3 \\
\hline Money (not relevant to gaming) & 3 & 3 & 4 & 4 & 3 & 2 \\
\hline Life as a whole & 4 & 3 & 4 & 4 & 2 & 3 \\
\hline Total score & 43 & 40 & 47 & 47 & 34 & 38 \\
\hline Total QOL points change & & -3 & & $\mathbf{0}$ & & +4 \\
\hline
\end{tabular}

$\mathrm{B}^{*}=$ Baseline score (1=poor, 2=fair, 3=good, 4=excellent). $\mathrm{A}^{* *}=$ After (end-point score).

Joy rated a loss of one QOL point, for the item of 'Living situation', bringing it into alignment with the assessment of her NOK, as being good. The RN however rated this item to be excellent. Joy also rated a loss of one point for the item of 'Family', although both the NOK and RN thought this item to be excellent. In terms of 'Life as a whole', Joy rated a loss of one point, bringing it into line with that of her NOK as being good. Joy often talked about her life, "I've been lucky...I have an excellent quality of life...I sometimes think I do have to die, but it's put aside, a little like the song Que Sera Sera" (I:26/1:1125, 102). The RN rated this item to be excellent on both assessments. The RN noted a slightly reduced score for 'Energy' and 'Self as a whole', which is likely reflective of a recent acute illness that resulted in referral for medical assessment. 
The biggest assessed change in QOL was from the NOK, rating a four-point improvement overall. Items improved were 'Physical health', 'Marriage/closest relationship', 'Self as a whole', 'Ability to do chores', 'Life as a whole', and 'Ability to have fun'. "She's making a point of going to all the activities, everything that is happening" (NOK:I:7/2:498). "It's less about her health and more about activities.... She comes down even without activities, talking and chatting" (RN:I:16/4:1453). "She used to poo-poo activities and it would take about a year or so for something to be accepted ... [gaming] is keeping her occupied” (NOK:I:7/2:500).

Two items had reduced scores by one QOL point at the final assessment according to the NOK: 'Friends' and 'Ability to manage money'. Joy often said to me that she no longer had friends as most of them had died, but she had acquaintances at Tenderlea, and even more so since gaming commenced, but they were not the same as friends (FN:24/4:650). The mention of friends connected her to her past life and friendships:

I was out with my friend and we saw these two Marines that we knew.... We chatted and that was that. A long time later, I saw him again and spoke to him. I tried to explain who I was....he said, 'that's impossible, she was beautiful!' [Joy was smiling and laughing]. I was happy that he thought I was once beautiful. I think it was my hairstyle; I had a pageboy at the time. (J:I:22/1:71)

\section{Case summary and postscript}

Joy had no conscious memory of computer-assisted exergaming, so every gaming session was a new adventure. She needed full assistance to engage with the gaming technology, but could follow cues and prompts, during which time she played with independence and free will. In almost all gaming sessions, she had gaming inertia, usually as a reaction to negative on-screen messages. However, gaming with the Able-X system was a catalyst for improved QOL for Joy. The most positive change effects were those relating to BPSD: remembering more and repeating less, less anxious and calmer, more likely to attend activities, especially new for her activities that required use of her hands, increased purposeful mobility, and less aimless pacing. Also, her revived motivation to re-engage and socialise with others during the gaming period, had an immediate QOL impact. Additionally, improvement in hand-eye coordination and pattern recognition were recorded by the Able-X computer-generated exergaming outputs. 
The above changes were maintained at 12 months post gaming according to the RN (I:18/7:1525) and affirmed by the NOK (I:15/7:1578). Of particular note was the maintained improvement in BPSD:

She occasionally repeats herself but not at the level of pre-gaming (RN:I:18/7:1529). She seldom if ever talks about being claustrophobic, which would be, related to anxiety...She has remained significantly happier since your gaming study began and her social skills remain very good. She also seems very comfortable among people she does not know. (NOK:I:15/7:1588, 1579)

Improved hand-eye coordination continued to be evident in daily life, the RN reported, "pre-gaming she was reluctant to use her hands for activities but is now bowling and doing crafts" (I:18/7:1535). The NOK had concurring evidence, "Joy is writing quite a lot of cards to family and friends without prompting, with little if any repetition...Her hand writing is remarkably steady" (I:15/7:1557). A final word from Joy: "I think who am I? Am I still that person? It's a complicated business having a brain and being a person [laughs]" (VN:8/4:1694).

\subsubsection{Iris $^{22}$}

She's like a flower. She just opens up when she feels the love. Physical contact, hugs and kisses are important to her. (NOK:I:16/1:359)

\section{Personal and clinical profile}

Iris was a tall, willowy octogenarian female. She enjoyed conversing in both English and her first language, Dutch. Married at the age of 23 and widowed in 2002 after 49 years of marriage, she kept a photograph of her late husband at her bedside and often repeated, "have you met my husband [name]...My husband is so nice, I love him" (RN:I:17/2:1241). On one occasion, during a rendition of 'have you met my husband', I said "no" and she smiled and sat on her bed, lifted her legs into the air and laughed like a

${ }^{22}$ Pseudonym: Iris means "Colourful” (http://www.thenamemeaning.com/iris/). 
teenager and said, "he's so handsome, I love him so much, he's a good man [her face blushed]" (FN:17/4:1581).

In April 2009, Iris became increasingly deluded, disabled, and confused. Her daughter recalled, "she would call the police believing people were stealing from her [misplaced things]" (NOK:I:18/1:4). These are typical non-cognitive behaviours common in dementia (Bpac ${ }^{\mathrm{nz}}$, 2013; Perkins, 2013; Prince et al., 2014; Small et al., 1997; Snowden et al., 2003). She had stopped using the oven and cooking for herself, which is indicative of instrumental functional decline (Stineman et al., 2014). She was often found wandering the streets day and night and family and friends were increasingly worried about her safety. Following a psychogeriatric assessment, a diagnosis of AD was made; subsequently she was prescribed donepezil (a cholinesterase inhibitor). Within a few days of diagnosis, Iris was admitted to a residential care bed at Tenderlea, a facility located $25 \mathrm{kms}$ from her home, but close to her daughter who was EPA for Personal Care and Welfare. Iris had been resident at Tenderlea for nearly five years when the study began.

My life is good. (I:I:14/4:1457)

In January 2014, Iris enjoyed excellent ambulation and independent mobility; however, some ADLs had become a challenge for her. Although independently mobile, she was no longer able to transport herself outside of the unit safely, resulting in restrictions to her ambulation and freedom, which she resisted. The RN recounted, "she tries to escape [and has a repetitive story to account for her need to leave]....Darlings, I have to go, you have to leave me alone...I need to find my Ma. I need to see if my house is burnt down or if there has been a flood or anything" (I:17/2:1249). She still had the ability to use cutlery to feed herself and therefore would be able to hold and use the Able$\mathrm{X}$ controller. Functional decline had extended to most other IADLs. She functioned at Stage 6 of the FAST (Reisberg, 1988, 2007) and the GDS (Auer \& Reisberg, 1997). Stage 6 is the penultimate dementia stage, where "individuals lose basic activities of daily life capabilities and in which behavioural disturbances peak in occurrence and severity" (Auer \& Reisberg, 1997, p. 168) and has been equated to a clinical diagnosis of moderately severe dementia (Reisberg, 2007). One week prior to commencement of gaming, Iris had been referred to the GP because "her friend had passed over... she has been really sad. She's just been sitting...before she was really lively" (RN:I:15/1:1326). This resulted in 
Iris being prescribed regular risperidone (antipsychotic medication). Up until this point risperidone was prescribed as and when required.

\section{Engagement in gaming}

Iris needed varying but near constant levels of assistance (every 5 to 10 seconds) to both engage with the computer-assisted exergaming technology and to make gaming progress (VL:4/2:AA000401:03:00). If she got stuck, she would turn her attention to whatever was on-screen or start to chatter, her late husband apparently referred to this as "her cocktail party persona" (NOK:I:14/4:1574). Every gaming session was not only a new experience for her, it was several new experiences or gaming moments, tenuously connected through the gaming technology (VL:3/2:AA000401:02:00). Gaming with Iris was constantly interjected by chatty communication, reminiscence (VL:28/1:AA000302:04:40) and lots of smiles and laughter as her concentration and focus wandered. When focused however, she could game with near constant verbal support. Iris played 8 of 12 solo gaming sessions over a 10-week period declining on four occasions, usually saying something like, "not at the moment darling ${ }^{23}$, I don't feel like playing" (I:I:14/4:1437). Iris played Circuit ( $n=6)$, Target $(n=8)$, Mosquito Splat $(n=4)$, Butterfly Catch $(n=4)$ and Popaloon $(n=5)$. Prior to gaming, she had never used a computer.

Gaming sessions varied in length depending on Iris' level of engagement and enjoyment. She played Circuit, Target, and Popaloon most often. Iris particularly enjoyed simple, time limited games, but as the games became more challenging, she tended to lose focus and start to chatter. The second reason for disengagement was the on-screen messages, both positive (enabling) and negative (disabling). Positive messages would trigger witty repartee, "I'm very clever, you love me don't you” (VN:23/3:1624), she was talking to the Able-X controller. Her pleasure state was etched on her face, smiling and laughing or in her relaxed and engaged body language (VN:31/3:2203). Positive messages also facilitated reminiscence, for example, well done, excellent, or similar words of encouragement either on-screen or from me, enabling connection to memories and stories about her late husband and children, "clever people...unlike me" (I:I:28/1:1846). Fun and laughter moments, of which there were many, connected her to memories of her Mum,

${ }^{23}$ Everyone was called darling as she had forgotten most names other than her children and husband. 
"my mum and I used to have lots of fun together, we used to laugh. It's nice having a mum like that" (VN:18/1:1825). Negative messages did the reverse.

Negative on-screen messages or noises disempowered Iris, resulting in disengagement and purposeful inaction (VL:4/2:AA000502:04:14). Comments such as too slow, try again, usually resulted in a rapid aversive response from Iris: "no, it's not my game" (VN:23/1:1675). "I don't see the fun in it" (VN:23/1:1670). "I am not clever" (VN:28/1:1843), and "I can't tell you why I don't feel clever, it's a pity, I wish I could" (I :I:28/1:1846). Meanwhile, during aversive moments, the game continues and the error messages start to stack up, making her more aversive. On one occasion, she slapped herself on the leg when the on-screen message 'too slow, try again' appeared (VN:4/2:2020). When her pleasure state was negative, it was obvious by her facial expression and body language, which often heralded verbalisation of the same. She would make every effort to break the news gently, as if not wanting to offend me by opting out of gaming (VN:7/4:2277). The hand held Able-X controller was the third cause for disengagement. It had three buttons on the top and a trigger, all of which competed for her attention and focus, creating distraction and disengagement (VN:16/4:1650). Regardless of distractions, over the weeks Iris and I played together, she enjoyed the gaming experience and this was affirmed by the clinical manager: "[She] always looks happy...coming out [of gaming]...I always ask what she did and if she enjoyed the games and she says yes" (M:I:5/2:706, 1062).

\section{Gaming outputs}

Iris' gaming ability improved in some challenges irrespective of her lack of ability to remember gaming previously and her short attention span. When comparing the baseline and end-point Circuit graph and scores, improvement in hand-eye coordination in terms of accuracy, speed and smoothness of movement is inferred (Figure 9). The biggest improvement was 17 points for accuracy, 15 points for speed and 12 points for smoothness. Conversely, on the game Target, which is specifically designed to develop primary skills of hand-eye coordination, she scored lower at end-point (-1040). Also, the heat map shows reduced activity at the peripheries at end-point assessment, other than the top left of the screen. 


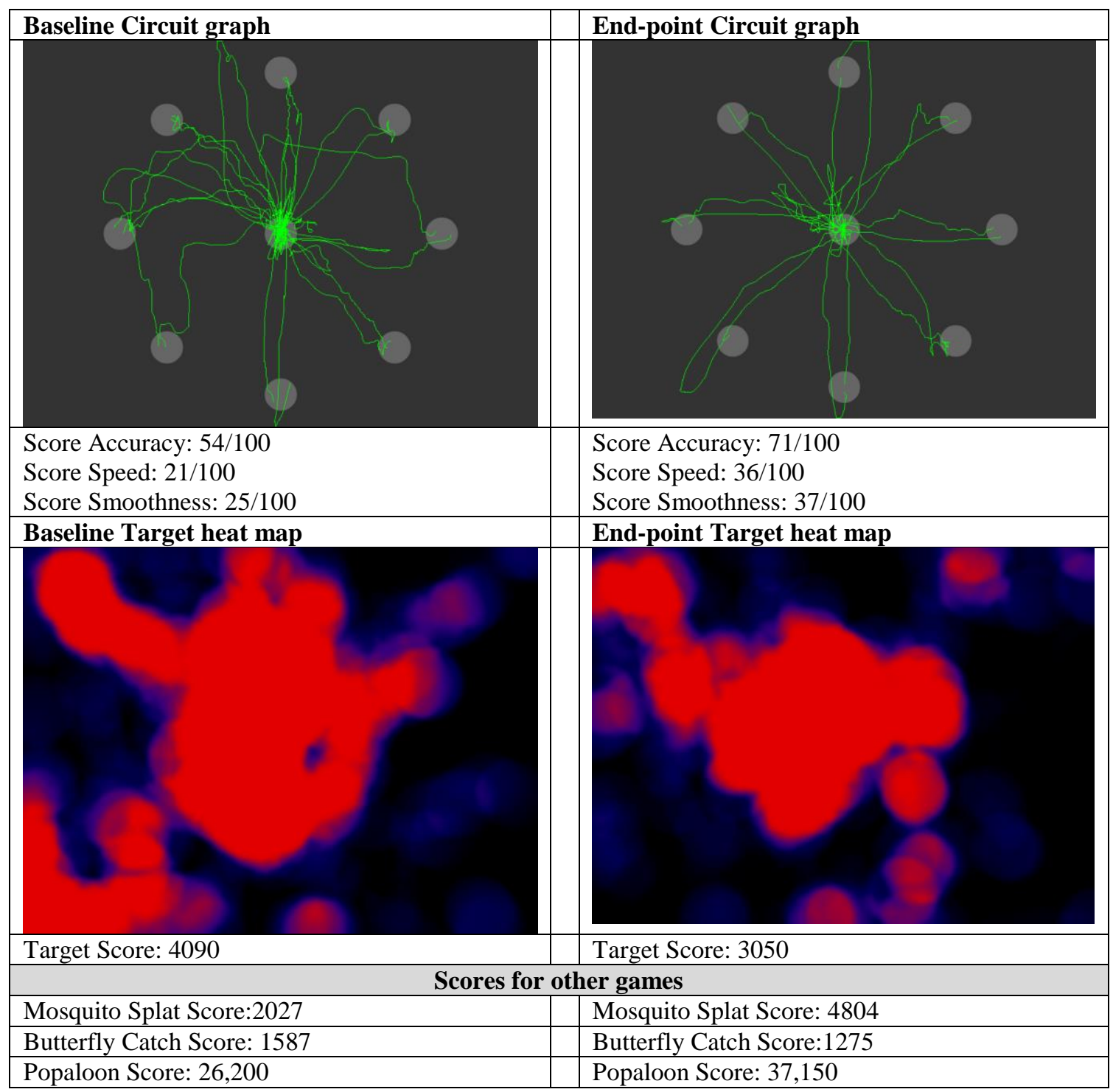

Figure 9. Iris: Computer-generated exergaming outputs

There was also variance in Iris' scores for Mosquito Splat and Butterfly Catch, the two games that introduce visual discrimination. For the former, she had an improved score of 2777 points, although she needed verbal assistance to enable her to discriminate between the mosquito and the butterflies in this game. For Butterfly Catch, she scored lower at end-point. Iris found the extra manipulation of catching a butterfly in her onscreen net and depositing it onto a flower difficult. In addition, as with Mosquito Splat, Iris was unable to discriminate, without assistance, between the on-screen targets and those to be avoided, so visual discrimination skills were not demonstrated in this case. Iris played well on Popaloon where her score improved by 10,950. In this case, it would be incorrect to claim improved concentration, as near constant verbal cues were needed. 
However, Iris was required to find the on-screen patterns herself and she did this very efficiently, demonstrating improved pattern recognition skills.

\section{Comparing baseline measures and end-point measures}

Iris' cognitive MMSE 2-BV improved by one point at the end of gaming (Table 13). Two points were acquired through improved orientation to time (month and season), however, one point was lost for orientation to place. Additional evidence of improved cognition during the gaming period was testified by others, "I've just spoken to Mum, she's back again, she was bright and clear" (NOK:I:10/2:758), the NOK is referring to a conversation she had with her sister (Iris' second daughter) who was unaware of the computer-assisted exergaming activities. The Manager recounted, "a staff member who resigned about three years ago...came to visit... and she [Iris] remembered, she said 'I know you'...How amazing is that”? (M:I:17/4:1122).

Table 13. Iris: Summary of baseline and end-point measures

\begin{tabular}{|l|r|r|}
\hline Validated measure & Baseline & End-point \\
\hline MMSE 2-BV & $5 / 16$ & $6 / 16$ \\
\hline RAID & $1 / 18$ & $1 / 18$ \\
\hline CSDD & $6 / 38$ & $1 / 38$ \\
\hline QOL-AD: Self-report & $41 / 52$ & $45 / 52$ \\
\hline QOL-AD: RN Proxy & $37 / 52$ & $47 / 52$ \\
\hline QOL-AD: NOK Proxy & $31 / 52$ & $33 / 52$ \\
\hline
\end{tabular}

There was no change in the already low RAID anxiety score, however, the RN reported, "she seems to be much more settled" (I:15/2:686) and she's "not wandering about as much" (I:16/4:938). However, the CSDD baseline score indicated a score of 6/38 and examination of the clinical record, signalled increasing episodes of depression over the previous three months, so the score was not surprising. Iris was also dealing with having recently lost her "special friend" (RN:I:16/4:949), resulting in conflict situations brought about by repeatedly visiting her late friend's room, which was now occupied by a new resident "Fortunately, quite a lot of times we managed to catch her before she went in” (M:17/4:1140). Iris commenced regular risperidone, for behavioural symptoms during the first week of gaming. By the end-point assessment, the CSDD score was reduced by five points, this reduction is likely a consequence of a combination of things, including coming to terms with the consequences of her loss, medication, gaming, and socialisation. 
By the end-point assessment, Iris had a new special friend. The manager also described indications of improved symptoms:

She was getting down into a depression I think... really depressed about it [friend's death] and then she came back right again. So, it's really hard to say if she had done it all on her own, or actually the therapy [computer gaming] helped that as well. And I think probably you should take some credit on that too. (M:I:17/4:1161)

QOL-AD reassessment revealed an overall increase of 16 points, and had an immediate positive impact on Iris' daily life. Iris self-reassessed an additional four QOL points in the items of: 'Energy', 'Ability to do chores', 'Ability to have fun', and 'Money' (Table 14).

Table 14. Iris: Quality of life points change pre-and post-gaming

\begin{tabular}{|c|c|c|c|c|c|c|}
\hline Items on the QOL-AD questionnaire & $\begin{array}{l}\text { Self } \\
\text { B }^{*}\end{array}$ & $\begin{array}{l}\text { Self } \\
\mathbf{A}^{* *}\end{array}$ & $\begin{array}{l}\text { RN } \\
\mathbf{B}^{*}\end{array}$ & $\begin{array}{l}\mathbf{R N} \\
\mathbf{A}^{* *} \\
\end{array}$ & $\begin{array}{l}\text { NOK } \\
\text { B }^{*}\end{array}$ & $\begin{array}{l}\text { NOK } \\
\text { A }^{* *}\end{array}$ \\
\hline Physical health & 4 & 4 & 3 & 3 & 3 & 3 \\
\hline Energy & 3 & 4 & 2 & 3 & 2 & 3 \\
\hline Mood & 3 & 3 & 2 & 4 & 3 & 3 \\
\hline Living situation & 3 & 3 & 4 & 4 & 4 & 3 \\
\hline Memory & 3 & 3 & 1 & 3 & 1 & 1 \\
\hline Family & 4 & 4 & 4 & 4 & 2 & 3 \\
\hline Marriage (closest relationship) & 4 & 4 & 4 & 4 & 3 & 3 \\
\hline Friends & 3 & 3 & 3 & 4 & 1 & 2 \\
\hline Self as a whole & 3 & 3 & 3 & 3 & 2 & 3 \\
\hline Ability to do chores & 3 & 4 & 2 & 3 & 2 & 2 \\
\hline Ability to do things for fun & 2 & 3 & 3 & 4 & 3 & 2 \\
\hline Money (not relevant to gaming) & 3 & 4 & 4 & 4 & 3 & 1 \\
\hline Life as a whole & 3 & 3 & 2 & 4 & 2 & 4 \\
\hline Total Score & 41 & 45 & 37 & 47 & 31 & 33 \\
\hline Total QOL points change & & +4 & & +10 & & +2 \\
\hline
\end{tabular}

$\mathrm{B}^{*}=$ Baseline score (1=poor, 2=fair, 3=good, 4=excellent). A A $^{* *}=\mathrm{After}$ (end-point score).

The RN agreed with all items of this self-reassessment, and overall awarded an additional 10 points, with two extra points for three items: 'Mood', 'Memory', and 'Life as a whole'. The NOK agreed that the item 'Energy' had improved, but the items 'Ability to have fun' and 'Money' had, in her opinion, deteriorated.

Evidence to support Iris' self-reassessment was recounted by others: "she is more lively... when she comes out of the session.... She is quite awake...It is not just me who observed it (M:I:5/2:702); "When she comes out...she is chatty and happy" (M:I 
5/2:1049). Also evidenced was increased interest and pride in managing her basic ADLs, particularly dressing and clothes selection. "When she is in a low mood, she dresses really down. She will wear pyjamas and stuff...but now she is choosing...brighter colours and will put on heaps of necklaces and things” (RN:I:15/2:690). I said to her one day, "you look gorgeous", she laughed and replied, "thank you darling, nice clothes make me feel good" (I:I:5/2:1104). The RN reported, "she particularly likes to be a wearable arts model, she loves to dress up" (RN:I:10/2:1277). "I let her choose her clothes, it gives her independence and makes her happy throughout the day" (RN:I:15/1:1371). The importance of clothing as an expression of selfhood and embodiment in dementia is explored by Twigg (2010) and clothing was very important to Iris. Additional QOL points were awarded by the RN and NOK for the item 'Friends'. "She has a new male friend. I don't think it is a gender thing...she just likes to have a close friend....She is a one friend person that she likes to focus on all the time" (RN:I:16/4:953). The remaining QOL items with improved end-point scores have been addressed under the MMSE-2:BV, RAID and CSDD section above.

\section{Case summary and postscript}

Iris had no conscious memory of gaming so every time we met was introductory and every game we played felt like a sequence of gaming moments and reminiscences. Although Iris needed verbal prompts every 5-10 seconds to be cognitively agent, she achieved many wonderful moments of independent gaming agency where she played with free will and was awarded improved gaming scores. She could independently use the Able-X controller when focused, but there were many moments where gaming focus was interrupted, usually by the on-screen messages and graphics that would trigger moments of reminiscence, self-reflection or gaming inertia.

Gaming with Able-X in partnership was a psychosocial intervention that resulted in improved QOL outcomes for Iris. The most positive changes were associated with improved energy levels and social re-engagement, along with improvement in some BPSD, such as, less unsettled behaviour and aimless wandering, and an improved depression score. She also demonstrated improved hand-eye coordination, which translated into daily life though a renewed interest in selecting her clothes, dressing up and jewellery selection. What was interesting in this case was how the Able-X suite of games on-screen graphics acted as a catalyst for reminiscence. 
Iris maintained improved BPSD and improved hand-eye coordination, which was evinced by the RN, "she dressed for the day and not for the night [and] the sorting of and the putting on of jewellery" (I:18/7:2296), for up to nine months post gaming following which there was a "sudden decline...her needs changed" (NOK:e-mail:12/7/15, p. 2). There was no secure bed available at Tenderlea, so her family took the opportunity to relocate Iris to a facility that was closer to family, where "She has settled well and they think she is adorable and declare she is never to leave" (NOK:e-mail:12/7/15, p.2). The NOK informant referred me to the RN for postscript follow up. The RN made no changes to the case transcript. A final word from Iris: "You are very lucky to be able to meet interesting people and play computer games with them" (I:I:16/1924). RIP February 2017.

\subsubsection{Bill $^{24}$}

A very compassionate, generous man (NOK:I:18/7:133). A gentleman...always trying to help, always trying to assist staff. He's happier on his own, and he's happy in the garden. (RN:I:11/7:776)

\section{Personal and clinical profile}

Bill was a tall, slim, septuagenarian male. Although Bill could speak, he seldom did, usually choosing appropriate monosyllabic responses when communicating. His wife affirmed, "he's never been a man for words" (NOK:I:18/7:191). Gradual loss of speech (dysphasia) is not uncommon in later stages of most dementias (Alzheimer's Society UK, 2013). His education concluded pre-high school level, leading to occupations in community patrol and as a detention warden. In 2010, at the age of 74, Bill's behaviour had become increasingly difficult for his wife to manage. He was refusing to shower or change his clothes, and stayed up most of the night "mucking around on his computer... [until the day] he couldn't remember how to use it....The next thing I know, he's taking all his computers and selling them for scrap" (NOK:I:23/8:1494). In 2011, aged 75 , he was wandering out of the house at night, which caused concern for his wife. "I was sleeping on the couch by the front door because I was worried he was going to take off" (NOK:I:18/7:104). Referral for assessment to a consultant geriatrician resulted in a diagnosis of Frontotemporal dementia. His wife recounted, "when he was 21 he had a

\footnotetext{
${ }^{24}$ Pseudonym: Bill means "Protection" (http://www.thenamemeaning.com/bill/).
} 
nervous breakdown and he went into [named] mental hospital... and they gave him shock treatment and I reckon that contributed to what's wrong with him now” (NOK:I:18/7:372).

By July 2012, at the age 76, Bill had become incontinent of urine and was resistive to care. The day and night reversal continued to manifest, "one night he got so violent and I was so frightened.... know it sounds horrible but the day the ambulance came and took him away it was a kind of relief" (NOK:I:18/7:83, 315). Following emergency assessment at the hospital, Bill was discharged to a secure dementia unit at Gardenside. The unit was in close proximity to his home, friends and family, including his wife who was also his NOK and EPA for Personal Care and Welfare. The closeness of Gardenside enabled Bill to remain socialised and active with his family, especially with his beloved grandson (Charlie, aged four) and his two dogs. At the time of the study, Bill had been resident in the dementia unit for nearly two years.

In July 2014, Bill was dependent on others for most ADLs and all IADLs. He had the ability to use cutlery to self-feed and could transfer and transport himself, the latter keeping him purposefully mobile and active within the confines of the bounded environment of the secure dementia unit. For all other ADLs and IADLs, he needed full assistance. Bill functioned at Stage 6 on the FAST (Reisberg, 1988, 2007) and the GDS (Auer \& Reisberg, 1997). Stage 6 is the penultimate dementia stage where "individuals lose basic activities of daily life capabilities and in which behavioural disturbances peak in occurrence and severity" (Auer \& Reisberg, 1997, p. 168) and has been equated to a clinical diagnosis of moderately severe dementia (Reisberg, 2007). Bill was prescribed seven regular medications, including paroxetine, an antidepressant for escalating noncognitive behaviours, such as "security patrolling... in the afternoons when the security guard in him comes out" (RN:I:6/8:2131). He was also prescribed PRN quetiapine, an antipsychotic, although it was not administered during the gaming period.

\section{Engagement in gaming}

Bill recalled playing the computer-assisted exergames, so each session was developmental, building upon previous gaming sessions. His prior experience of computer gaming was obvious, although he had lost IADLs and needed assistance to engage with the computer, Able-X gaming suite, the controller and to initiate gaming. Once initiated, he played all 12 gaming sessions, seven solo and five group sessions, over a four-week 
period. He played: Circuit $(n=12)$, Target $(n=12)$, Mosquito Splat $(n=12)$, Butterfly Catch $(\mathrm{n}=12)$ and Popaloon $(\mathrm{n}=12)$.

\section{Solo gaming sessions}

Gaming sessions with Bill followed the pre-set routine 'Cognitive Challenge'. Once connected to the gaming technology, he could follow instructions and quickly moved to independent gaming agency with minimal verbal prompting. He looked at one with the technology and was a confident gamer. If he needed assistance, he made gaming noises such as "nooo", "ooh", "aah" and "yeah" to communicate (VL:13/8:48.00). I noted that some of the games appeared to be too easy for him and recommended that we make them more challenging. Bill declined, preferring to enjoy success at the current challenge level. Positive on-screen messages made him smile. Verbal praise and encouragement made him laugh and increasingly vocal with an array of gaming noises. Bill very rarely received negative messages so was rarely influenced by them, but when he did, his demeanour became agitated and annoyed (VL:13/8:28:00). He enjoyed independent gaming and his enjoyment extended to enjoyment by association in the group sessions, as long as they were filled with success and excitement.

Bill occasionally became disengaged from gaming for one of three reasons. The first was the drift associated with the Able-X controller, which he found distracting. I was not successful in teaching him how to re-centre it, so I would try to centre it for him, but he found this assistive touch intrusive and would pull his hands and arms away causing more disengagement. I knew from my experience with other participants that he would likely adjust his play and self-compensate for the drift, and he did. By gaming session six, he had absorbed the drift into his game and, gaming with the drift seemed to add a level of challenge that was acceptable to him and appeared to augment the gaming experience and increase the physicality of gaming, as upper body movements became more exaggerated (VL:12/8:37:37). Secondly, Bill had an innate desire to use the trigger on the controller, even on games where it was not needed; this slowed his gaming progress, and was the second cause of disengagement.

Thirdly, Bill's level of wakefulness impacted on gaming engagement. In three of the five group sessions Bill had moments where he appeared to be sleeping and therefore disengaged from the gaming experience. However, when other participants were being 
successful, he would watch, enjoy, and support with encouraging noises and the occasional word. If gaming was less animated and/or interrupted by negative on-screen messages and noises, or if continuance of gaming required constant verbal instructions, Bill would fold his arms, close his eyes and occasionally fall asleep. When awoken, he was always sufficiently alert to re-engage in gaming and would smile at the inference that he had fallen asleep, shaking his head as if to infer he was just resting his eyes.

However, it was the presence of his wife and grandson (Charlie) in the gaming room when Bill's gaming was most animated. His whole body and mind appeared more alert and his verbal communication improved markedly, announcing on one occasion, "This is my grandson" (VN:19/8:2676). The ambience and energy levels were enriched through intergenerational relations, similar to the finding of Galbraith et al. (2015) and George and Whitehouse (2011). All present benefitted by association (VL:12/8: 30:00), especially in the presence of Charlie, who on one occasion shushed the members of the group because the toy he had brought in to the gaming room was 'sleeping'. All participants obeyed and quietly stifled giggles and exchanged glances. Charlie continued with his conversation with Granddad in a whispered voice, whilst crunching on his snacks (VN:18/6:2682) and for several moments the gaming room was very quiet, other than the sound of Charlie's crunching. When they left, the special body language, facial alertness and smile that Bill had shown in their presence, left with them and only reappeared during 'exciting' group gaming sessions.

\section{Group gaming sessions}

Group sessions involved three participants (two males, one female), plus one observer (wife of one participants who was also a resident in the dementia unit) and on four occasions, Bill's wife and grandson. One of the benefits of group gaming was the enjoyment of gaming through social association. Bill appeared to get pleasure from observing the success of other players, especially Thomas, who was also a very good player. As previously stated, Bill disengaged when the ambience of the group gaming room was less animated and/or interrupted by negative on-screen messages and noises. However, Bill achieved consistently improved scores (Figure 10). 


\section{Gaming outputs}

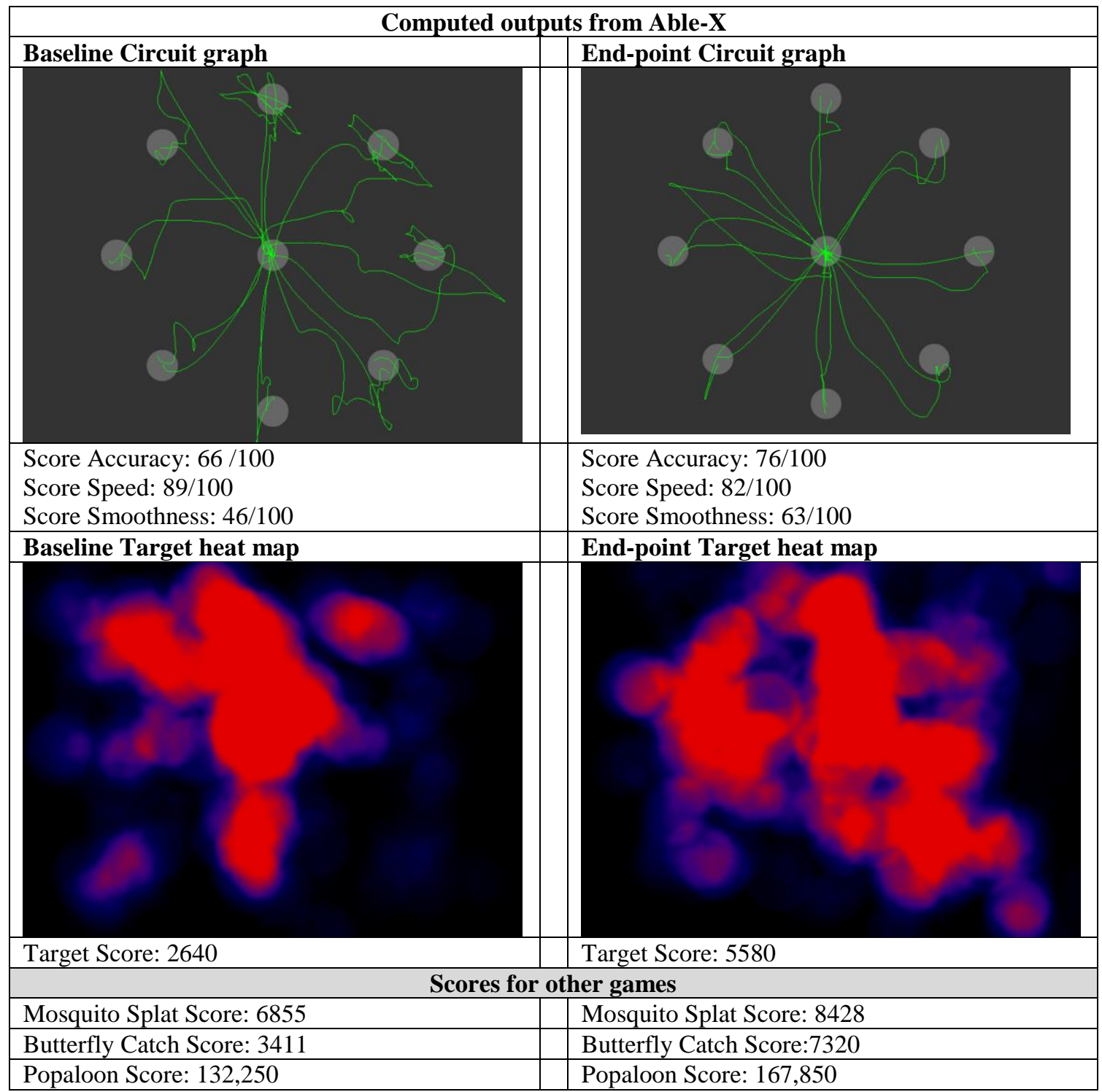

Figure 10. Bill: Computer-generated exergaming outputs

Bill's ability to game and follow on-screen prompts facilitated independent gaming and improved gaming scores. A comparison of baseline and end-point Circuit graph and scores imply improved hand-eye coordination in terms of improved scores for accuracy by 10 points and smoothness by 17 points. Although Bill's speed score reduced slightly by 7 points, he was a very fast gamer from day one, and as he reduced his speed, his accuracy and smoothness improved over time. Improved activity was also evident on the Target challenge scores and heat maps, further evincing improved hand-eye coordination. Initially, Bill's gaming focused on centre and upper left of the Target screen. By the end of gaming, he was seeing and hitting targets in a wider area, particularly in the middle and on the right of the screen. His end-point score for Target was improved by 2940 points. 
Also, improved were his scores for Mosquito Splat, Butterfly Catch, and Popaloon. For Mosquito Splat and Butterfly Catch, Bill needed verbal reminders to avoid non-target objects, so improvement in visual discrimination was not established. However, Bill's improved score of 35,600 for Popaloon, evinced improved concentration and pattern recognition. Also, worthy of note was his excellent gaming performance and style (VL:20/8:55:28).

\section{Comparing baseline measures and end-point measures}

Bill's cognitive score according to the MMSE-2:BV reduced by three points (Table 15), which was not unexpected in the penultimate Stage of global functional decline and where MMSE score of < than 5 are not unusual (Reisberg, 1988; Auer \& Reisberg, 1997). He lost two points in registration and recall, and another point for orientation to time.

Table 15. Bill: Summary of baseline and end-point measures

\begin{tabular}{|l|r|r|}
\hline Validated measure & Baseline & End-point \\
\hline MMSE 2-BV & $6 / 16$ & $3 / 16$ \\
\hline RAID & $4 / 18$ & $2 / 18$ \\
\hline CSDD & $3 / 38$ & $1 / 38$ \\
\hline QOL-AD: Self-report & $39 / 52$ & $38 / 52$ \\
\hline QOL-AD: RN Proxy & $35 / 52$ & $43 / 52$ \\
\hline QOL-AD: NOK Proxy & $37 / 52$ & $38 / 52$ \\
\hline
\end{tabular}

Of the three dementia unit participants, Bill was the only one who remembered gaming when asked, "They [group participants] come back [from gaming] really alert and bright....[Bill] is the only one that remembered at the end of the day that he went gaming", (RN:I:21/8:1646, 1740). Improved cognition was inferred by the computerised output scores for the exergame Popaloon, which were indicative of improved concentration and pattern recognition skills over the gaming period.

Although cognitive score deteriorated, the end-point scores were more positive for other dimensions of QOL, for example, reduced scores by two points each for the RAID and CSDD measures indicate positive change. Other evidence of reduced anxiety and depression include, "I think he's a lot calmer but then of course he's on his medication" (NOK:I:23/8:1356), and "he's much more relaxed...calmer once he's had the stimulation....[He is] less stressed...much more placid, much more relaxed" (RN:I:21/8:1714, 1727). Conversely, if something happens he did not like "he'll run off 
and chase them" (RN:I:21/8:1728). This latter comment could be evidence of escalating functional decline and deteriorating BPSD, not uncommon at this Stage of global decline (Auer \& Reisberg, 1997). However, the RN stated that Bill had also started "patrolling of the corridor outside his room" (RN:I:21/8:1605), inferring this was a problem. Bill spent most of his working life as a community patrol officer and a warden at a detention centre, and the patrolling behaviour could be explained as Bill drawing on his prior experience of being agent in the field.

Following completion of the 12 gaming sessions, overall QOL had improved by eight points. Bill's self-reassessment for QOL-AD was reduced by one point. However, according to others his QOL had improved by one point according to the NOK and by eight points according to the RN (Table 16):

Table 16. Bill: Quality of life points change pre-and post-gaming

\begin{tabular}{|c|c|c|c|c|c|c|}
\hline Items on the QOL-AD questionnaire & $\begin{array}{l}\text { Self } \\
\text { B }^{*}\end{array}$ & $\begin{array}{l}\text { Self } \\
\mathbf{A}^{* * *}\end{array}$ & $\begin{array}{l}\mathbf{R N} \\
\mathbf{B}^{*}\end{array}$ & $\begin{array}{l}\mathbf{R N} \\
\mathbf{A}^{* *}\end{array}$ & $\begin{array}{l}\text { NOK } \\
\text { B }^{*}\end{array}$ & $\begin{array}{l}\text { NOK } \\
\mathbf{A}^{* * *}\end{array}$ \\
\hline Physical health & 3 & 3 & 2 & 3 & 3 & 3 \\
\hline Energy & 4 & 3 & 2 & 3 & 3 & 2 \\
\hline Mood & 3 & 3 & 2 & 3 & 3 & 3 \\
\hline Living situation & 1 & 3 & 3 & 3 & 3 & 3 \\
\hline Memory & 3 & 3 & 1 & 3 & 2 & 3 \\
\hline Family & 3 & 3 & 4 & 4 & 4 & 4 \\
\hline Marriage (closest relationship) & 3 & 3 & 4 & 4 & 4 & 4 \\
\hline Friends & 3 & 3 & 2 & 3 & 3 & 4 \\
\hline Self as a whole & 3 & 3 & 3 & 4 & 3 & 3 \\
\hline Ability to do chores & 3 & 3 & 4 & 4 & 3 & 2 \\
\hline Ability to do things for fun & 3 & 3 & 4 & 4 & 2 & 3 \\
\hline Money (not relevant to gaming) & 4 & 2 & 1 & 1 & 1 & 1 \\
\hline Life as a whole & 3 & 3 & 2 & 4 & 3 & 3 \\
\hline Total Score & 39 & 38 & 35 & 43 & 37 & 38 \\
\hline Total QOL points change & & -1 & & +8 & & +1 \\
\hline
\end{tabular}

$\mathrm{B}^{*}=$ Baseline score (1=poor, 2=fair, 3=good, 4=excellent). $\mathrm{A}^{* *}=$ After (end-point score).

Bill gave himself extra points for 'Living situation', bringing his score into alignment with the scores of the NOK and RN for this item. Both the NOK and RN agreed that Bill's 'Memory' had improved, bringing their scores into alignment with Bill's own assessment on this item, but contrasts with the MMSE-2:BV score. There was agreement that his relationship with 'Friends' had improved. Additional evidence of this was reported by the $\mathrm{RN}$, "they [group participants] all kind of sit together in the...[recreation] room" (RN $\mathrm{I}: 21 / 8: 1690)$. This is a change for Bill, who prior to gaming preferred his own company 
(RN:I:11/7:776). Increased socialisation had an immediate and sustained impact on Bill's QOL.

The NOK reported additional improvement of one QOL point for Bill's ‘Ability to do things for fun', "I know he does look forward to it [gaming]” (NOK:I:23/8:1372). The RN reported improvements in 'Mood', 'Self as a whole' and 'Life as a whole', acknowledging that "he seems more alert, and happy when he comes out [of gaming]" (RN:I:21/8:1687). Additional evidence for this element is presented under anxiety and depression scores above. Finally, the RN rated a one-point improvement for 'Physical health', however, other than the computer-generated printouts that inferred improved hand-eye coordination; there were no other reports to support improved physical health during the gaming period. Both Bill and his NOK reported reduced 'Energy' levels'; the RN, however, thought Bill's 'Energy levels' had improved a little. Variable energy levels and the above QOL changes could be explained by the introduction of mood altering medication prescribed during the gaming period. Whatever the reason, Bill's quality of life improved by eight points overall between baseline and end-point assessments.

\section{Case summary and postscript}

Bill could recall gaming with Able-X and was quick to achieve independent gaming agency and improvement. He needed assistance to initiate and move from game to game, minimal verbal instruction and occasional recalibration of the drift, associated with the Able-X controller, which occasionally halted gaming flow, as did his variable levels of wakefulness. Although Bill found the Cognitive Challenge easy, he was reluctant to move to the next level of challenge. The gaming experience for Bill was either in individual gaming sessions or in small group sessions (2-3 participants). He was at his most animated when his wife (NOK) and grandson were present, and in small group situations, where he could enjoy, by association, the gaming success of others. However, group gaming could also result in Bill becoming disengaged, particularly if the gaming environment lacked excitement. Computer-assisted exergaming with Able-X was a catalyst for improvements in QOL for Bill. The most positive change for him was the development of new friendships that extended beyond the gaming environment and back into the dementia unit. Also, at a time when his BPSD was escalating due to the disease process, there was evidence he was less restless and more settled, wakeful and aware in 
the afternoons on gaming days. Furthermore, the computer-generated gaming outputs revealed improvement in hand-eye coordination and pattern recognition.

The three most important aspects in Bill's case are the concept of continued/repetitive success at the most basic gaming level being preferred to the risk of not being as successful at a more challenging gaming level, expanded social and friendship network and improvement in some aspects of BPSD at a time when these symptoms were escalating due to the disease process. In the three months following gaming, "his new friendships... and socialisation ...continued" (RN:I: 28/11:2168). The facility replaced computer-assisted exergaming sessions with other stimulating activities to capitalise on the "content calmness that extended into the late afternoon [which followed gaming with Able-X]” (RN:I:28/7:2177). Activities included adult colouring books, bingo, singing, reminiscence, music and karaoke, all of which had "the same effect of extending calm and happy behaviour and reduced pacing and unsettled behaviour in the afternoon and evenings [similar to effects following gaming with Able-X] (RN:I:28/7:2129).

The RN was of the opinion that improved hand-eye coordination that was acquired through gaming with Able-X "facilitated continuance of activities such as painting, colouring, gardening, dressing and feeding himself, up to ... [his death]", three months after gaming concluded. Although she did not wish to be interviewed, Bill's NOK read and concurred with the above improvements. She made one correction to the CS transcript; regarding the number of medications Bill was prescribed. Final words and actions from Bill: "This is my grandson [he was smiling with pride and hugging Charlie close to his chest]”(VN:19/8:2676). RIP November 2014.

\subsubsection{Thomas ${ }^{25}$}

He's still got his sense of humour...he cracks little jokes all the time, the staff quite like him for that....He's a lot more agreeable now when told to do things, whereas when my mother started with Alzheimer's he was reluctant to go along with anything he didn't agree with... he would fight the authorities quite forcefully. He's become more docile. (NOK:I:18/7:828)

\footnotetext{
${ }^{25}$ Pseudonym: Thomas means “A Twin” (http://www.thenamemeaning.com/thomas/).
} 


\section{Personal and clinical profile}

Thomas was of average height, slim, octogenarian, and male. He was born in England and emigrated to NZ in 1991. When World War II broke out he joined the Navy and was trained as a mechanic to work on aircraft. Post war he worked as an office clerk in a steel mill, during which time he took night classes and became a qualified accountant. His son recounted, "he dragged us up another status symbol in the class structure" (NOK:I:18/7:1200).

In 2012, at the age of 85, Thomas was struggling to cope at home with his wife, who had advancing AD, so they gave up their home and went to live in a local rest home. In the same year, family members noticed that Thomas was exhibiting some short-term memory deficits. Following an assessment by a geriatrician, he was also diagnosed with AD. In 2013, Thomas was separated from his wife as her advancing dementia resulted in her requiring a more secure environment, which was not available at the rest home of their choice.

It was during these weeks of separation that Thomas' own mental and functional capacity rapidly deteriorated to such an extent that he could no longer cope without assistance. 'He was wandering around [rest home] saying 'have you seen my wife' and all this sort of thing...It was taking months [to get him a bed near his wife]...it's easier to get someone in a rest home in Russia I think, than transfer them between [in NZ]" (NOK:I:18/7:899). In January 2014, a bed became available in Gardenside, the residential unit attached to where his wife was accommodated, and Thomas relocated there. However, Thomas continued to decline, constantly searching for his wife. In February 2014, he was transferred to the secure dementia unit section of Gardenside and finally reunited with his wife of nearly 60 years. Gardenside was in close proximity to his son, who was EPA for Personal Care and Welfare, and was NOK. At the time of this study, Thomas had been resident in the dementia unit for five months. The change helped, " $h e$ 's got his wife with him, and they walk around the unit hand in hand. They have breakfast, lunch and dinner together" (RN:I:11/7:667).

In July 2014, Thomas was independent but supervised with ADLs. IADLs living were more of a trial for him. He was very mobile around the small dementia unit and 
equally small garden area, always with his wife and constant companion. He functioned at Stage 5 on the FAST (Reisberg, 1988, 2007) and the GDS (Auer \& Reisberg, 1997). Stage 5 is where "individuals can no longer survive if left on their own" (p. 168) and has been equated to a clinical diagnosis of moderate dementia (Reisberg, 2007).

\section{Engagement in gaming}

Thomas could not recall gaming at the start of each session, so games commenced with an introductory revision element. He needed assistance to connect with the technology, but could follow instructions, both on-screen and verbal. Once gaming, he played with independent gaming agency and rarely needed verbal prompting from me. Thomas had experience of using computers, although he had never played computer games. He seemed to be very comfortable with the Able-X controller and gaming technology, in fact, he had the best gaming technique of all participants (VL:18/8:15:22). He had an innate skill of incorporating the drift associated with the controller into his game. Thomas played all 12 gaming sessions, four solo and eight group sessions, over a four-week period. He played: Circuit $(n=12)$, Target $(n=12)$, Mosquito Splat $(n=12)$, Butterfly Catch $(n=12)$ and Popaloon $(n=12)$.

\section{Solo gaming sessions}

If Thomas needed assistance during gaming, he would make a witty comment rather than ask for help. His NOK reported, "if he's joking and he's not moaning he's generally happy" (I:18/7:1005). I noted immediately that some of the games appeared to be too easy for him, but on enquiry he said, "I enjoy the game... it is not too easy or too hard" (VN:11/8:2702), so gaming continued at the most basic level. Thomas could read the on-screen messages and the positive messages made him smile and laugh. They also motivated him, as did positive praise and encouragement from others. He rarely attracted negative on-screen messages, but did attract negative noises on Mosquito Splat and Butterfly Catch, as he was unable to avoid non-target objects without verbal prompting from me. The negative noises would trigger a witty comment such as, "get out of here" (VN:5/8:2476), adding to the pleasure and enjoyment by association of others present, with only minimal loss of gaming focus (VL:5/8:19:29). He was a very charismatic gamer, for example, on one occasion, he was playing so hard his glasses steamed up and interrupted gaming, much to the enjoyment of all present. He was also a very selfcompetitive player (VL:29/7:18:03) and he enjoyed looking at his gaming scores and 
statistics (VL:5/8:13:13). Thomas's wife was always present during the gaming sessions; they spent every minute of every day together.

\section{Group gaming sessions}

Group sessions were made up of two or three participants, Bill and Maya, plus Thomas' wife. One of the benefits of group gaming was the enjoyment of gaming through social association and Thomas was influential in this aspect (VL:19a/8:01: 03:45). Thomas appeared to get gaming pleasure from observing the success of other players, one in particular (Bill), who was also a very good player. Thomas was attentive to all other players, offering encouragement, through supportive gaming words and noises, for example, he would mimic the noise of the on-screen mosquito and other on-screen critters.

Thomas enjoyed wonderful support from his beloved wife. She would read out the on-screen messages and constantly congratulate him on his success, announcing to all, "he is good, I know that. I am used to it" (VN:11/8:2668). Thomas responded by making a fist, gestured a punch in his wife's direction, and said, "I am working on my reaction time" (VN:11/8:2671). His NOK affirmed his sense of humour, "if he's on an even keel and he's cracking jokes he's happy” (I:18/7:1009).

Rarely did Thomas disengage from gaming but when he did, it was his response to negative on-screen noises, and occasional lack of focus. The latter was always a shift of focus from gaming to his wife. If he could not see, hear or feel her, he would stop gaming to check on her (VL:4/8:36:00, VL:6/8:06:20). To ensure her inclusion he would often give up his gaming seat, resulting in episodes akin to the game musical chairs (VL:12/8:11:05). This slight disruption was nothing compared to the independent agency it triggered in his wife and another female participant (Maya). Thomas was very gallant and the women hung on to his every word.

\section{Gaming outputs}

Thomas' ability to game and follow on-screen prompts enabled independent gaming agency and improved gaming scores in every game, other than the game Popaloon (Figure 11). 


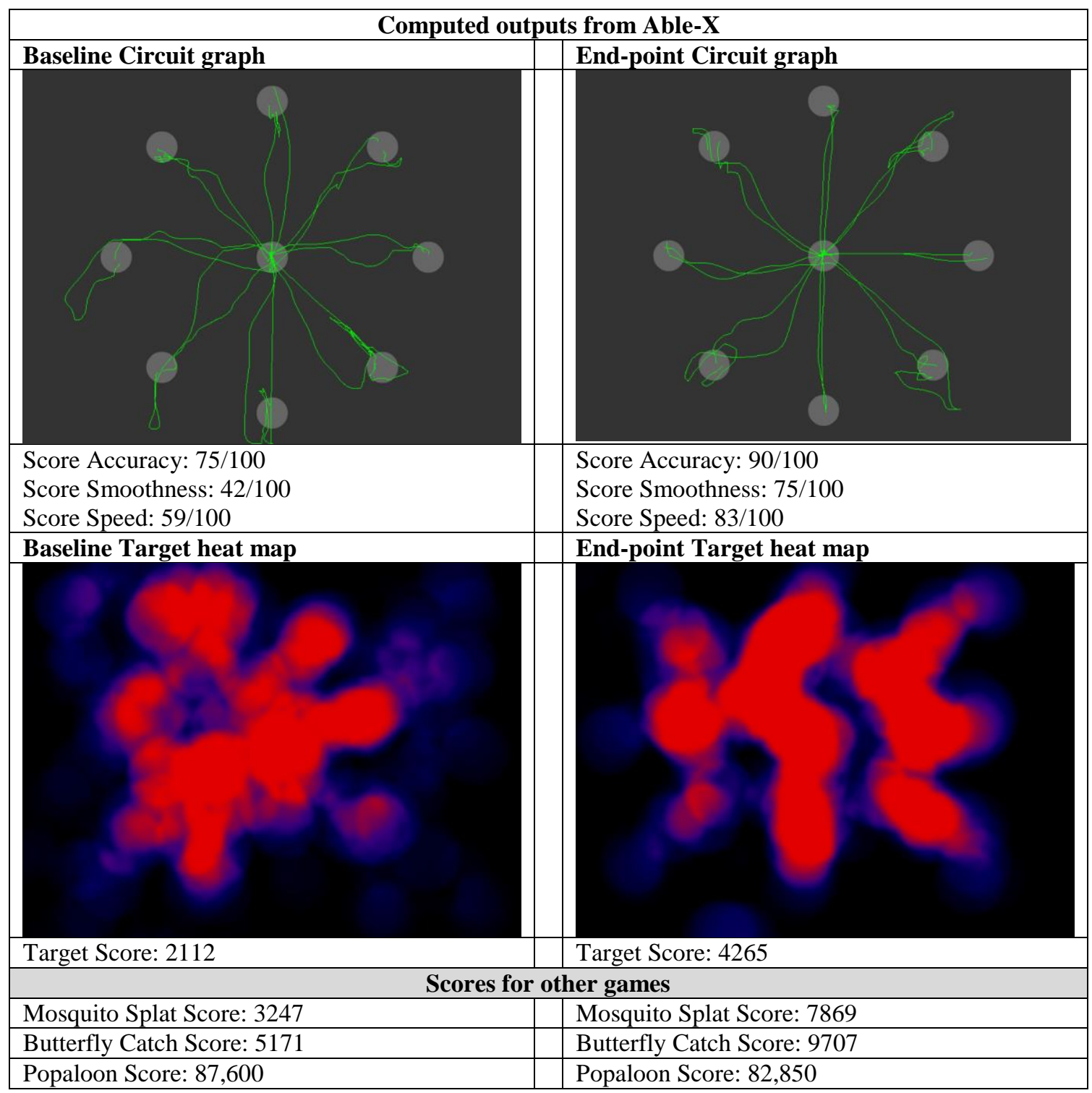

Figure 11. Thomas: Computer-generated exergaming outputs

When comparing the baseline and end-point Circuit graph and scores, improvement in accuracy by 15 points, smoothness by 33 points and speed to target by 24 points, all of which inferred improved hand-eye coordination. Improvements were also demonstrated on the Target challenge heat maps and score. Initially, Thomas' gaming focused on centre and upper middle of the screen. By the end-point, he had a wider gaming field, particularly on the right, but did not particularly improve in the peripheries of the gaming screen. His end-point score for this game more than doubled, further evincing improved hand-eye coordination. Thomas improved his scores on Mosquito Splat by 4622 and Butterfly Catch by 4536, however, Thomas needed verbal reminders to avoid non-target objects, so improvement in visual discrimination was not established in this case. For the game 
Popaloon, his score reduced by 4750 points and he needed occasional verbal prompting to progress, so there is no claim of improved concentration. However, his gaming technique and ability to find on-screen patterns was a pleasure to watch (VL:20/8:01:01:00).

\section{Comparing baseline measures and end-point measures}

Thomas' cognitive score of 4/16 was unchanged at the end-point according to MMSE 2-BV (Table 17). This low MMSE score is unusual at Stage 5 of global decline, it is more in keeping with Stage 6 according to the FAST (Reisberg, 1988) and the GDS (Auer \& Reisberg, 1997).

Table 17. Thomas: Summary of baseline and end-point measures

\begin{tabular}{|l|r|r|}
\hline Validated measure & Baseline & End-point \\
\hline MMSE 2-BV & $4 / 16$ & $4 / 16$ \\
\hline RAID & $3 / 18$ & $1 / 18$ \\
\hline CSDD & $2 / 38$ & $0 / 38$ \\
\hline QOL-AD: Self-report & $40 / 52$ & $39 / 52$ \\
\hline QOL-AD: RN Proxy & $43 / 52$ & $45 / 52$ \\
\hline QOL-AD: NOK Proxy & $35 / 52$ & $34 / 52$ \\
\hline
\end{tabular}

Although his cognitive score did not improve, it was maintained. Maintenance is one of the goals of non-pharmacological interventions for dementia, to slow cognitive and functional decline (Yamaguchi et al., 2010). Other positive changes in cognitive state were reported: "He's a lot more oriented...I'm noticing a bit more of him trying to orientate [his wife]" (RN:I:6/8:1311, 1315), and that he is "more alert...at the end of the day" (RN:I:21/8:1613). The NOK reported, "Dad in particular seemed a little more sort of, aware than usual" (I:11/8:1457). Gaming output scores did not reveal additional cognitive improvements in concentration or pattern recognition in this case.

Reduced scores by two points each for the RAID (anxiety) and CSDD (depression) indicated immediate improved QOL in these areas, although the scores were very low at baseline. Other evidence of reduced anxiety and depression include improvements in the wider aspects of BPSD:

He's more calm [since gaming] and then she's calmer [wife] and so it's a knockon effect...They'd have ding dong arguments in the hallway, we just called them marriage tiffs...but we're having none of that now (RN:I:6/8:1359, 1336)...He's 
less anxious in the afternoon...much calmer between 4 and 5 [pm, and] less likely to get agitated by other people. (RN:I:21/8:1656, 1668)

Thomas' end-point scores were more positive for some of the other QOL measures (Table 18). The QOL-AD end-point self-reassessment by Thomas was reduced by one point for both 'Memory' and 'Family'. The RN scored additional points for both aspects, implying his memory was good and his relationship with family was excellent, however, she did not offer additional evidence to support her assessment, whereas Thomas did, which evinces insight: "My memory's going...[it is] pretty poor at the moment" (T:I:22/8:1874, 1894). When asked about family Thomas reflected, "I haven't had much contact with them, since I've been in here” (T:I:22/8:1897), a view supported by his NOK: "I have [visited] a little bit yeah...not as much as I would've liked to [less than his usual weekly visit due to work commitments]" (I:11/8:1431).

Table 18. Thomas: Quality of life points change pre-and post-gaming

\begin{tabular}{|c|c|c|c|c|c|c|}
\hline Items on the QOL-AD questionnaire & $\begin{array}{l}\text { Self } \\
\text { B }^{*}\end{array}$ & $\begin{array}{l}\text { Self } \\
\mathbf{A}^{* * *} \\
\end{array}$ & $\begin{array}{l}\text { RN } \\
\mathbf{B}^{*}\end{array}$ & $\begin{array}{l}\mathbf{R N} \\
\mathbf{A}^{* *} \\
\end{array}$ & $\begin{array}{l}\text { NOK } \\
\text { B }^{*}\end{array}$ & $\begin{array}{l}\text { NOK } \\
\text { A }^{* *}\end{array}$ \\
\hline Physical health & 3 & 3 & 4 & 4 & 3 & 3 \\
\hline Energy & 3 & 3 & 4 & 4 & 3 & 3 \\
\hline Mood & 3 & 3 & 4 & 3 & 3 & 3 \\
\hline Living situation & 3 & 3 & 4 & 4 & 3 & 3 \\
\hline Memory & 2 & 1 & 1 & 3 & 2 & 2 \\
\hline Family & 4 & 3 & 3 & 4 & 3 & 3 \\
\hline Marriage (closest relationship) & 4 & 4 & 4 & 4 & 3 & 3 \\
\hline Friends & 3 & 3 & 2 & 4 & 2 & 2 \\
\hline Self as a whole & 3 & 3 & 4 & 4 & 3 & 3 \\
\hline Ability to do chores & 3 & 3 & 4 & 2 & 3 & 2 \\
\hline Ability to do things for fun & 3 & 3 & 4 & 4 & 3 & 3 \\
\hline Money (not relevant to gaming) & 3 & 4 & 1 & 1 & 1 & 1 \\
\hline Life as a whole & 3 & 3 & 4 & 4 & 3 & 3 \\
\hline Total Score & 40 & 39 & 43 & 45 & 35 & 34 \\
\hline Total QOL points change & & -1 & & +2 & & -1 \\
\hline
\end{tabular}

$\mathrm{B}^{*}=$ Baseline score (1=poor, 2=fair, 3=good, 4=excellent). A**=After (end-point score).

Relationships with 'Friends' were improved according to the RN by two points. The RN also reported "the small group [group participants] continue to socialise and sit together in the activities room" (I:28/7:2239), demonstrating that new friendships and associations had extended beyond the gaming room. Both the RN and NOK rated Thomas' 'Ability to do chores' had deteriorated over the previous few weeks, but provided no evidence to support this. Thomas rated no change in this item and thought his abilities were good. The final negative change since gaming according to the RN was loss of one 
point for 'Mood', indicating his mood was now good rather than excellent, this assessment is aligned to the ratings of Thomas and the NOK, who reported "[in] the last two weeks [his mood] has been good" (I:11/8:1508).

\section{Case summary and postscript}

Thomas was unable to recall gaming with Able-X from day-to-day. He needed assistance to connect with the technology, initiate gaming and to move between games, but once gaming, he did so with independent gaming agency and minimal verbal prompting, usually when the skill of visual discrimination was needed. The two main detractors for Thomas, were the negative on-screen noises and the proximity of his wife in the gaming space. In group sessions, there was a 30-second window of opportunity to self-initiate, that appeared to be related to repetitive exposure to gaming instructions. Although Thomas found the Cognitive Challenge easy, he was reluctant to move to the next level. He was more interested in self-competition to improve his own scores, and in enjoying the consistency of success that the gaming experience offered him. Thomas was inspiring to observe and his gaming technique was exemplary. With his natural gaming charisma, he could heighten the ambience of the gaming experience for all present.

Computer-assisted exergaming with Able-X was a catalyst for improvements in QOL for Thomas; the most positive change was improvement in cognitive orientation and alertness following gaming. Thomas also showed signs of improvement in behaviours associated with BPSD such as reduced anxiety and agitation and extended periods of calmness in the afternoon. Friendships were extended during the gaming period and sustained in non-gaming moments in the dementia unit. As in other cases, Thomas' computer-generated outputs indicated improved hand-eye coordination. Finally, this case elucidated the concept of 'gaming by association'. Thomas demonstrated gaming technique, success and charisma through self-competition and his positive appraisal of the gaming of others, all of which had an undoubted positive impact on others present.

All the above improvements were maintained for six months post gaming, following which Thomas had a period of gradual and then more rapid decline "which left him sub where he was pre-gaming by July 2015" (RN:I:28/11:2232) in terms of cognitive and physical functioning. At postscript (11 months after gaming intervention), improved BPSD and friendships were maintained (RN:I:28/11:2236). Final words from RN: “They 
[Thomas and wife] joke at each other, they laugh, they walk hand-in-hand... and they're always together, there is never one without the other" (I:21/8:1616). RIP September 2015. RIP wife October 2016.

\subsubsection{Maya $^{26}$}

One of the most loving women you'd find, she'd help everybody....In the ward there they call her Mum, because she helps them with the others. (NOK:I:14/7:263)

\section{Personal and clinical profile}

Maya was average height and build, septuagenarian and female. In 2010, at the age of 73, she was showing signs of early cognitive decline. "I could see her starting to go...her father had it" (NOK:I:14/7:178). She had been visiting the doctor for some time, but "she never...told him about things...I had to go in with her and explain" [what was going on] (NOK:I:14/7:211). She was forgetting things, "[we would] sit down...just have a chat and she'd say, 'have you seen my Mum and Dad lately'?" (NOK:I:14/7:331). Maya was referred to a consultant geriatrician who diagnosed AD. "She was given a pill [donepezil]...to slow it down...but [they] told us there was nothing else they could do" (NOK:I14/7:186,189). She was also prescribed quetiapine on an 'as required basis', but it was not administered during this study.

By 2013, she required constant supervision. In early 2014, "[She] put a casserole dish on the top of the stove instead of in the oven....I heard a BANG...I go out there...all slivers of glass all over the kitchen, food all over" (NOK:I:14/7:436). No longer feeling able to cope, a request for assistance resulted in rapid admission into a secure dementia unit at Gardenside. The accommodation was 20 kilometres from her family, which enabled Maya to remain socialised and active with her family on a daily basis. At the start of this study, Maya had been resident in Gardenside for three months.

She's got a bloody excellent quality of life here....She's getting everything else she needs and wants...I couldn't supervise her constantly at home like... [they] do

\footnotetext{
${ }^{26}$ Pseudonym: Maya means "Mother" (http://www.thenamemeaning.com/maya/).
} 
here.... I thought I'd get used to coming in to see her and going, but I cried last night, too bloody emotional. (NOK:I:14/7:714, 487)

In July 2014, Maya was independently mobile both within the secure boundaries of the dementia unit, and in the daily supervised outings with family members (husband and children). She could manage some ADLs (eating, ambulating), for others she needed supervision and assistance. However, IADLs were increasingly difficult for her. Her NOK reported that she could not use her debit card or mobile phone, and although she enjoyed baking, "she was forgetting stuff so then...I was making sure that she had all the ingredients to go in" (NOK:I:14/7:402). She functioned at Stage 6 on the FAST (Reisberg, 1988, 2007) and the GDS (Auer \& Reisberg, 1997). Stage 6 is the penultimate dementia stage where "individuals lose basic activities of daily life capabilities and in which behavioural disturbances peak in occurrence and severity" (Auer \& Reisberg, 1997, p. 168) and has been equated to clinical diagnosis of moderately severe dementia (Reisberg, 2007).

\section{Engagement in gaming}

Maya had never used a computer before, so needed near constant verbal assistance to engage with the gaming technology and to make gaming progress. Each gaming session was as if starting all over again, as her ability to recall previous gaming sessions was variable. Maya gamed according to the Cognitive Challenge routine. She played all 12 gaming sessions, six solo and six group sessions over a four-week period. She played: Circuit $(n=12)$, Target $(n=12)$, Mosquito Splat $(n=12)$, Butterfly Catch $(n=12)$ and Popaloon $(n=12)$.

\section{Solo gaming sessions}

Once gaming, Maya needed prompting every 5-10 seconds to remain gaming and cognitively agent (VL:4/8: 02:38). In the first few sessions, she found it difficult to accept praise. If I said she was doing really well she would say, "I don't think so" (VN:29/7:2117), or “thank you” (VN:28/7:2079), the latter being her most used word. She would grimace when I read the positive on-screen comments. Her ability to follow verbal instructions contemporaneously, was rewarded with positive on-screen messages and improving scores, which, over time, made this very shy and reserved lady laugh out 
loud and blush (VL:18/8:48:16). Actual visual proof (contemporaneous gaming scores) that she was improving, resulted in gaming confidence. As her confidence and enjoyment escalated, so did her need to connect with others in the moments of gaming success, as she sought validation in their eyes and faces, as if seeking proof from me and others that she had reason to be pleased and proud of herself (VL:19/8:49:10).

Maya seemed to be comfortable with the Able-X controller and gaming technology, she also had a very economical gaming technique (VL:19a:01:10:36). Although distracted by the trigger, she did not seem to notice the drift associated with the Able-X controller, and would have continued to play on in error without assistance. Constant assistance was wearying for me as gaming partner. However, the wearying effects were attenuated in the group situation where there were multiple assistive voices of encouragement.

Facilitating gaming with non-participant observers in the room could enhance or undermine the gaming experience. In this case, there were moments of both, for example, on one occasion, the atmosphere of the gaming room was enriched as another participant (Thomas), and his wife (non-participant observer) entered. Maya was very pleased to see her friends. She gave Thomas the biggest smile and patted him on the leg. She verbally greeted his wife who patted Maya on the shoulder. They were momentarily joined as a trio by tactile connection and reunion. It is 20 minutes since they last met (VN:19a/8:2796). On other occasions, the presence of a non-participant observer was undermining, for example, on two occasions a non-participant observer joined the gaming session and observer would encourage her when she was successful, but if she made gaming errors, observer would use language such as, "you missed it...you silly girl" (VL:11/8:13:45, 13:38), resulting in disengagement.

\section{Group gaming sessions}

Gaming in groups provided opportunities to listen to gaming instructions several times and watch others play, creating a gaming revision scenario for game watchers. These revision sessions appeared to prepare Maya for gaming. Group sessions also enabled engagement and gaming by association (VL:19a/8:01:15:58, 01:27:40). Although Maya was unable to read the on-screen messages when gaming herself, she could and did read them for others when she was game watching. With near constant direction, her 
motivation and gaming improved. It was wonderful to see her transition on a continuum from denial of ability for gaming, to celebration with smiles, laughter, selfacknowledgement and confidence, resulting in many joyous partnership and group gaming moments (FN:20/8:3018).

The main reason for disengagement during gaming for Maya was the trigger on the Able-X controller. She was tempted to use it on most games and needed constant assistance to readjust her grip to avoid it. On Popaloon, the game where the trigger is required, she could press it, but constantly forgot that she had to release the trigger for it to remain active. Another reason for disengagement was the negative feedback from others. One of the differences in Maya's case was her reaction to negative on-screen messages and noises, which, in contrast to all other players, made her laugh, and resulted in momentary gaming inertia or screen watching as she purveyed the one-screen blood and gore (VN:19/8:2890).

\section{Gaming outputs}

Maya's ability to follow verbal instructions contemporaneously empowered her to game in partnership, and through this partnership, she was rewarded with improved scores in every game (Figure 12). A comparison of baseline and end-point Circuit graph and scores reveal improvements in all three aspects of accuracy, smoothness, and speed, which implied improved hand-eye coordination. Supporting this implication are improvements in the Target challenge heat maps and score. Initially, Maya's gaming focused on centre and upper middle of the screen. By the end-point, she had a different gaming field, particularly on the right side of the screen; however, the left middle peripheral field looked neglected. Her end-point score for this game improved by 1130 points.

Scores for Mosquito Splat, Butterfly Catch, and Popaloon were improved by 4764 for Mosquito Splat and 2950 for Butterfly Catch. However, she needed near constant verbal reminders to avoid non-target objects, so improvement in visual discrimination was not established. For the game Popaloon, a substantial improvement of 21,050 points was achieved. Like the former two games, Maya played with the support of constant prompting and occasional adjusting of her handgrip on the Able-X controller to release the trigger. Maya tended to point and fire at all targets irrespective of pattern matching, so there is no claim of improved cognitive skills of concentration or pattern recognition in this case. 


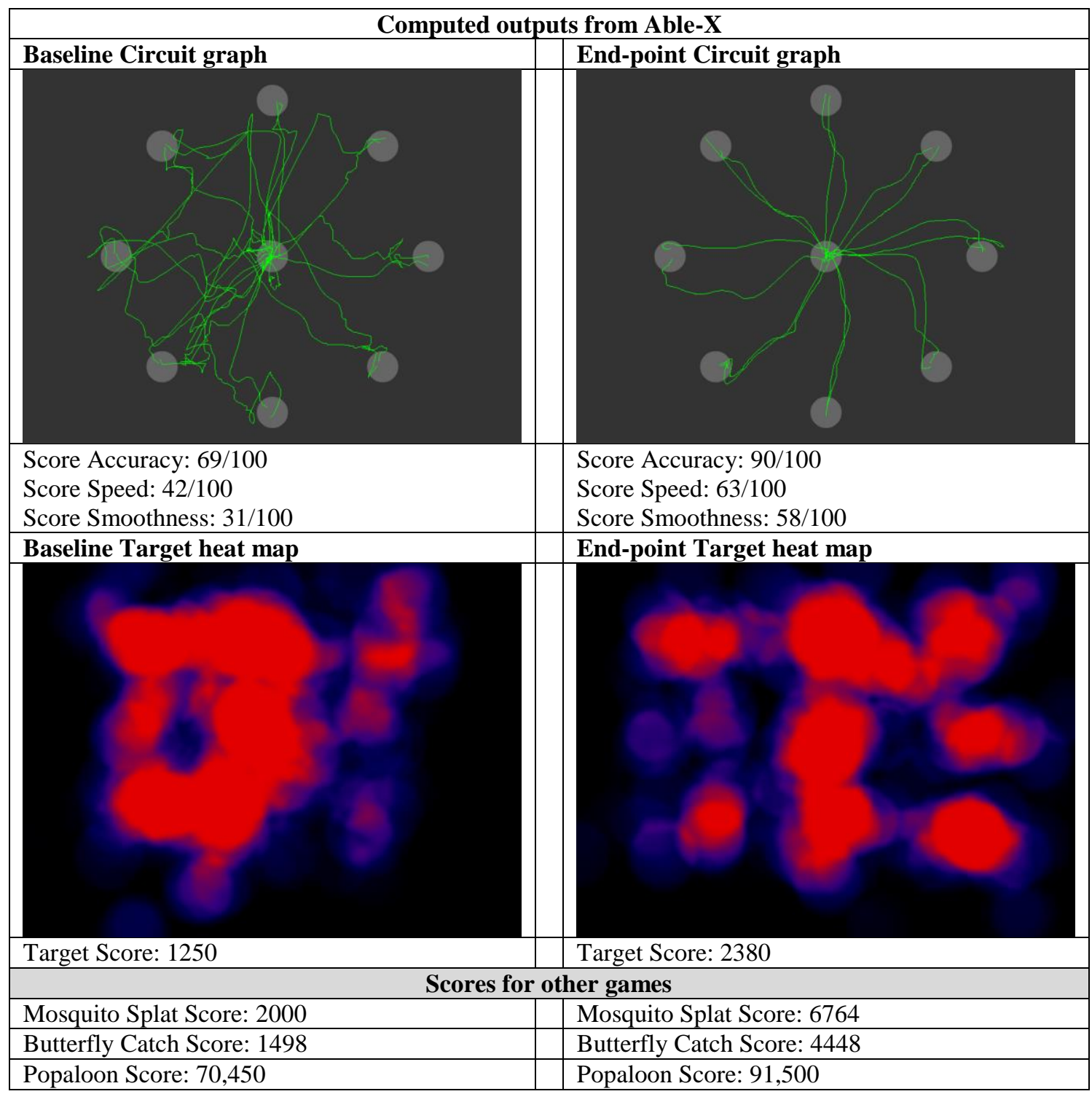

Figure 12. Maya: Computer-generated exergaming outputs

\section{Comparing baseline measures and end-point measures}

Maya's cognitive score improved by three points over the course of the study (Table 19). She could register and immediately repeat three words. Other evidence of improved cognition was reported by the $\mathrm{RN}$, "[Maya is] more alert...very receptive. The other day she did all the breakfast dishes" (I:21/8:1407). Prior to gaming constant instruction about each item to be cleared from the table was required. 
Table 19. Maya: Summary of baseline and end-point measures

\begin{tabular}{|l|r|r|}
\hline Validated measure & Baseline & End-point \\
\hline MMSE 2-BV & $1 / 16$ & $4 / 16$ \\
\hline RAID & $0 / 18$ & $0 / 18$ \\
\hline CSDD & $0 / 38$ & $2 / 38$ \\
\hline QOL-AD: Self-report & $42 / 52$ & $39 / 52$ \\
\hline QOL-AD: RN Proxy & $41 / 52$ & $46 / 52$ \\
\hline QOL-AD: NOK Proxy & $39 / 52$ & $39 / 52$ \\
\hline
\end{tabular}

In this case, there was no evidence of anxiety in dementia according to the RAID score and only a slight increase in the depression score at end-point assessment according to the CSDD. The latter score increased by two points, which related to the item of weight loss in the last month, which was due to a purposeful reduction in "junk food" (RN:I:21/8/:1533). The NOK and RN awarded extra QOL points for 'Physical health', which could be attributed to this purposeful weight loss and improved diet, rating this item as excellent at end-point. However, Maya rated this item unchanged and good. Maya thought her 'Mood' was reduced slightly at end-point, rating it good. However, her NOK and $\mathrm{RN}$ rated no change for this item rating it as good and excellent.

Others indicated improvements in some BPSD, which are often associated with anxiety and depression for example:

She seems more settled now and calmer...I don't think she gets as agitated (NOK:I:22/8:1961). When she's agitated she'd be folding her clothes ... and pacing around....She's happier and more settled.... When she comes out [of gaming] she's quite settled.... I did notice that, she's not looking to go home, especially in the morning (DT:I:6/8:961). She's happy and she's more relaxed....not going pacing, [or] looking for ways to go home or get out. (RN:I:21/8:1425).

In this case, there is no quantitative improvement evident in terms of reduced anxiety and/or depression. However, there is reported evidence of improvement in other aspects of BPSD, including less pacing and trying to leave the unit, less restlessness and less clingy/shadowing. Also, Maya is calmer, happier, and more social and settled following gaming with Able-X, all of which contributed to an improved QOL. 
In terms of overall QOL at end-point, there was variance between the ratings of Maya, the RN, and the NOK. Maya inferred her 'Overall QOL' had reduced by three points. However, according to the RN it had improved by five QOL points. Maya's NOK's reassessment score was unchanged (Table 20).

Table 20. Maya: Quality of life points change pre-and post-gaming

\begin{tabular}{|c|c|c|c|c|c|c|}
\hline Items on the QOL-AD questionnaire & $\begin{array}{l}\text { Self } \\
B^{*}\end{array}$ & $\begin{array}{l}\text { Self } \\
A^{* * *}\end{array}$ & $\begin{array}{l}\mathbf{R N} \\
\mathbf{B}^{*}\end{array}$ & $\begin{array}{l}\mathbf{R N} \\
\mathbf{A}^{* * *}\end{array}$ & $\begin{array}{l}\text { NOK } \\
\text { B }^{*}\end{array}$ & $\begin{array}{l}\text { NOK } \\
A^{* * *}\end{array}$ \\
\hline Physical health & 3 & 3 & 3 & 4 & 3 & 4 \\
\hline Energy & 2 & 3 & 3 & 4 & 3 & 4 \\
\hline Mood & 4 & 3 & 4 & 4 & 3 & 3 \\
\hline Living situation & 3 & 3 & 3 & 4 & 1 & 4 \\
\hline Memory & 3 & 3 & 1 & 2 & 3 & 1 \\
\hline Family & 4 & 3 & 4 & 4 & 3 & 3 \\
\hline Marriage (closest relationship) & 3 & 3 & 4 & 4 & 4 & 3 \\
\hline Friends & 3 & 3 & 4 & 3 & 3 & 3 \\
\hline Self as a whole & 3 & 3 & 3 & 4 & 4 & 3 \\
\hline Ability to do chores & 4 & 3 & 4 & 4 & 4 & 3 \\
\hline Ability to do things for fun & 3 & 3 & 4 & 4 & 3 & 4 \\
\hline Money (not relevant to gaming) & 3 & 3 & 1 & 1 & 1 & 1 \\
\hline Life as a whole & 4 & 3 & 3 & 4 & 4 & 3 \\
\hline Total Score & 42 & 39 & 41 & 46 & 39 & 39 \\
\hline Total QOL points change & & -3 & & +5 & & $\mathbf{0}$ \\
\hline
\end{tabular}

$\mathrm{B}^{*}=$ Baseline score (1=poor, 2=fair, 3=good, 4=excellent). A**=After (end-point score).

The range of the scores is 39-46 out of a total of 52, implying that all agree Maya has a good to excellent 'Overall QOL' at both baseline and end-point assessment. The biggest change in terms of points according to Maya's NOK was 'Living situation', which he rated as excellent at end-point. "I know she's got a bloody excellent quality of life here" (NOK:I:14/7:714). The RN agreed and rated this item excellent, adding, "she's happy and she's more relaxed...[she is] participating...and laughing with other residents" (RN:I:21/8:1421). Maya self-rated this item unchanged and good.

According to the 'Memory' aspect on the QOL-AD scale, Maya self-rated this item as unchanged and good at end-point and the RN rated it to be slightly improved and fair, however, the NOK reported loss of QOL points, rating 'Memory' as poor at the end-point assessment, revealing variance across raters regarding this item. All three raters agreed that the end-point rating for 'Family', 'Friends', 'Energy', and 'Ability to do things for fun' was good or excellent. However, there was more variance across the raters regarding 'Ability to do chores' and 'Life as a whole'. Maya rated her relationship with 'Family as slightly reduced at end-point and good. Her NOK agreed that it was good but unchanged. 
The RN however, thought this item unchanged and excellent on both assessments. Maya and NOK rated 'Friends' as unchanged and good. However, the RN thought this item to be slightly reduced at end-point implying that Maya was choosing to spend more time with her gaming colleagues, "they [group participants] all kind of sit together as well in the rec [recreation] room" (RN I:21/8:1690), therefore spending less time socialising with others in the unit.

Regarding the item 'Energy', Maya rated herself to have a little more energy, rating this item as good at end-point, and the RN and NOK concurred with this improvement, rating this item as excellent. "She appears much more active and engaged...especially when her group of friends are present” (RN:I:28/7:2037). All agreed that she had good or excellent 'Ability to do things for fun'. However, according to Maya her 'Ability to do chores' and 'Life as a whole' were reduced by one point, rating both these items as good. Her NOK concurred, but the RN thought these items were unchanged or improved, rating them both excellent at end-point.

In terms of the item of 'Marriage/closest relationship' there was variance between raters. Maya rated these items unchanged and good. The RN considered this item unchanged and excellent. However, Maya's NOK reported loss of one point at end-point rating this item as good. His reduced rating could be due to his distress caused by Maya no longer recognising him as her husband. "Just now, she still said 'that's my dad' [NOK sobbing]...[she is] proud as punch though [sob]" (NOK:I:14/7:270). The RN reiterates this reality, "She thinks her husband is her parent [father]. She gets quite upset with him... [if] she thinks he is doing things he [dad] shouldn't be doing [if he tried to kiss and cuddle her] (RN:I:11/7:66, 124). Anger on the part of family, close relatives and caregivers and relationship changes is not unexpected at this late stage of the dementia journey (Mittelman et al., 2004; Røsvik et al., 2011, 2013). Maya is trying to live well now (Oyebode \& Parveen, 2016) by reinventing her family in a contemporary space and time through sensory perception (Millett, 2011). She was overtly creating her new world and naming the members closest to her. One of her gaming partners and his wife became her cousins and another participant became her grandad (RN:I:21/8:1348, 26). On one occasion, when we were walking to the gaming room Maya called out, "come on grandad”, Bill replied, "hey”, he looked at me, shrugged his shoulders and rolled his eyes, 
and then made eye contact with Maya and smiled as he shook his head from side to side, a sort of reluctant acceptance of his new title (VN:18/8:2686).

\section{Case summary and postscript}

Maya was unable to recall gaming with Able-X from day-to-day and needed constant (every 5-10 seconds) assistance to cognitive gaming agency. However, as the small gaming group matured, other participants would encourage her to gaming agency and therefore slightly reducing the need for my input, as other participants became increasingly agent and confident. Maya was at her most animated in group sessions, which also facilitated her to agency through repetition and revision through game watching. She was at her most disempowered in the presence of negative verbal or body language, which on a few occasions resulted in aversive behaviour towards gaming.

Computer-assisted exergaming with Able-X was a catalyst for improvements in QOL for Maya. The most positive change was improved BPSD, including reduced agitation, pacing and attempts to leave the unit and increased moments of being calm, relaxed, happy and settled. Also noted were new associations that extended beyond the gaming room, which were maintained within the dementia unit following gaming activity. As with the previous four cases, Maya's scores are indicative of improved hand-eye coordination. In addition, by the end of the gaming period, others report that she is more cognitively alert, bright, and receptive to instruction, which was demonstrated through her ability to complete a sequential task such as clearing the breakfast tables. What is different about this case is that Maya adopted the on-screen positive messages to use as her repertoire when encouraging and motivating others.

Some of the above improvements were maintained at 12 months post gaming. Improvements in BPSD were maintained, "she is not pacing, agitated or looking to escape" (RN:I:28/7:2049). In terms of improved hand-eye coordination, the RN reported this to have continued as evidenced by, "activities such as setting the table...colouring books [new activity] and dressing herself [relearned activity]" (RN:I:28/7:2061). New friendships and associations were maintained and developed:

Excitement and enjoyment has continued, especially if her friends are present. She continues to be much more active and engaged (RN:I:28/7:2029). Nearly every 
resident has been given a family title, brother, sister, uncle, aunt, husband. The latter title has had a negative impact on her husband who sees it as a betrayal. He is her dad in her new family. He visits less frequently now and is angry about the situation. (RN:I:28/7:2054)

Maya did not appear to notice Bill's absence, however, and she did not reassign the title of granddad to anyone in her new family. She relearned the ability to complete a whole task (clearing the breakfast/dinner table); however, this ability diminished six months post gaming. In addition, there was no evidence that the language adopted from the gaming screen was ever used beyond the gaming room. Final word from Maya: “Thank you”(VN:28/7:2079). RIP May 2016.

\subsection{6 $\operatorname{Risa}^{27}$}

She's an extrovert personality... in that she is...bright and interested and likes to be centre of attention and...to be important. Her short-term memory is nonexistent ....two minutes later she forgets. (NOK:I:26/7:576)

\section{Personal and clinical profile}

Risa was an average height and built, octogenarian (83), female. She told me, "I don't like to up myself but...I get on with people [and] I enjoy life....Not bad for a 92-yearold eh" (VN:30/7:1842). She presented as rather dramatic and very artistic and this was evidenced through her activities of playing the piano, singing, and painting. In 2012, Risa was depressed and lonely in the home she had shared with her late husband, whom she described as "a beautiful man and good looking too... a great father...I was lucky to have him so long" (I:22/7:317). Her daughter, who was also EPA for Personal Care and Welfare, was concerned, as she "was getting thinner. The Meals on Wheels ${ }^{28}$ that were being provided for her often hadn't been eaten and she would go out and buy stuff and it would still be in the cupboard" (NOK:I:26/7:689). She was also leaving the house unlocked and walking to the shops, usually finding her way home, but not directly. "I didn't know whether she was actually looking after herself....There was no way she would

\footnotetext{
${ }^{27}$ Risa: Laughter (http://www.thenamemeaning.com/risa/).

${ }^{28}$ Meals on Wheels is a community service providing pre-cooked meals daily or as ordered.
} 
let me come and oversee that...It's quite distressing seeing that lost and...startled sort of look" (NOK:I:23/71652), although Risa "would deny...vehemently that there was anything wrong” (NOK:I:26/7:710).

Relief for the family came in 2013 , when Risa decided to put her name down on the waiting list for a residential apartment at Gardenside, where she had been attending as a volunteer during the day, providing ministry sessions and leading sing-alongs. Risa had been resident in Gardenside for 16 months when this study commenced.

When my husband passed, I decided to come here for the day...It's very homely and I got used to the staff...I'm glad I did it, you've got company and good meals and things and you go out...It was a good move. (R:I:22/7:308)

In July 2014, Risa enjoyed good functionality. Her mobility was excellent with the support of a walking stick that helped steady her during bouts of "raging tinnitus" (R:I:22/7:1077), for which she was prescribed prochlorperazine maleate ${ }^{29} \mathrm{PRN}$, an antipsychotic medication (not administered during gaming period). Although she had lost most IADLs abilities, including preparing meals, managing money and using a cell phone, she had retained some instrumental skills such as selecting her clothes, keeping her room organised and using the remote control for the television. She was fully independent with all basic ADLs if she was given the time to do them, otherwise "she would get anxious...when ...rushed...[or] out of her comfort zone” (RN:I:22/7:469). According to the FAST (Reisberg, 1988, 2007) and the GDS (Auer \& Reisberg, 1997), she functioned at Stage 4, a stage where "Individuals generally have deficit in management of instrumental activities of daily life" (Auer \& Reisberg, 1997, p. 168), which equates to a clinical diagnosis of mild dementia (Reisberg, 2007). Risa's family disagreed with the diagnosis of $\mathrm{VaD}$. "I struggle with this concept of dementia because the only thing that I see...is ...memory loss” (NOK:I:26/7:580).

\section{Engagement in gaming}

Risa had previously used a computer for word processing but not computer gaming. She needed varying levels of assistance to both engage with the computer-assisted exergaming technology and to make gaming progress. Every gaming session was a new

\footnotetext{
${ }^{29}$ Prochlorperazine maleate can have a slightly sedative and mood altering effect (Medsafe, 2014d).
} 
experience for her, but once into the games she had some degree of remembrance that she had played the game before (VL:29/7:00:05), which triggered gaming confidence. Although she needed near constant verbal prompting and physical recalibrating of the Able-X controller to achieve gaming agency, the effort of assisting her was balanced by the pleasure and ambience created in the gaming environment through her animated and dramatic gaming style. Gaming with Risa was constantly interposed with moments of surprise and laughter as her exaggerated reactions to gaming made us both jittery at times (VL:29/7:13:56: VL:5/8:12:05). Risa was the most animated and vocal player of all the participants. Once gaming, I understood what her NOK meant when she described her as, "slightly exaggerated...animated... [and] dramatic" (NOK:I:26/7:599). Risa played 12 solo gaming sessions during which she played: Circuit $(n=12)$, Target $(n=12)$, Mosquito Splat $(n=12)$, Butterfly Catch $(n=10)$, and Popaloon $(n=12)$.

From the first gaming session, it appeared that some of the games were too easy for her, but when I suggested that I should increase the challenge level, she frowned and said, "It was hard enough" (VN:29/7:1710). If I reduced verbal feedback she would be lost and a little panicked (VL:11/8:30:00). She had capability to ask appropriate questions to elicit additional information, and the positive on-screen messages and words of encouragement facilitated motivation and the growth of gaming confidence. Her NOK reported, "She is enjoying the games [they are]...making her brain work (NOK:I:6/8:1941). Risa said that gaming is "interesting [and it] makes your brain work very quickly” (VN:30/7:1825).

Disengagement from gaming was triggered by two gaming situations. One was her exaggerated response to negative gaming noises, for example, the splat noise associated with hitting a non-target image or, as Risa would describe, "murdering” nontarget images such as butterflies or bees (VN:6/8:1932). This situation interrupted gaming to allow her to gather gaming intelligence. She would ask questions to improve her game and to reduce the collateral damage on-screen. The second reason for disengagement is related to the first, in that she would assess the gaming screen with the eye of an artist and receive the gaming noises with the ear of a musician and comment on the on-screen splat gore of error play, which would then connect her to her memories of her art work and painting. She likened the Circuit path traces (Figure 13), to the movement of an artist arm and hand, as she demonstrated her technique with her outstretched arm (VL:12/8:03:55). 
The musical sounds of gaming linked her to memories of when she used to sing at weddings (VL:29/7:5:00). Unlike some participants, Risa did not appear to be unduly hampered by the drift associated with the Able-X controller; she would adapt her play to accommodate it. On one occasion, the controller was pointing directly at me, at virtual right angle to the gaming screen. She played on, laughed, and stated, "I feel as though I am going to shoot you" (VL:28/7:16:00).

\section{Gaming outputs}

Although some of the games appeared too easy for Risa, her variable gaming outputs suggest otherwise. A comparison of baseline and end-point Circuit graph demonstrates improved hand-eye coordination in terms of accuracy, speed, and smoothness of movement (Figure 13).

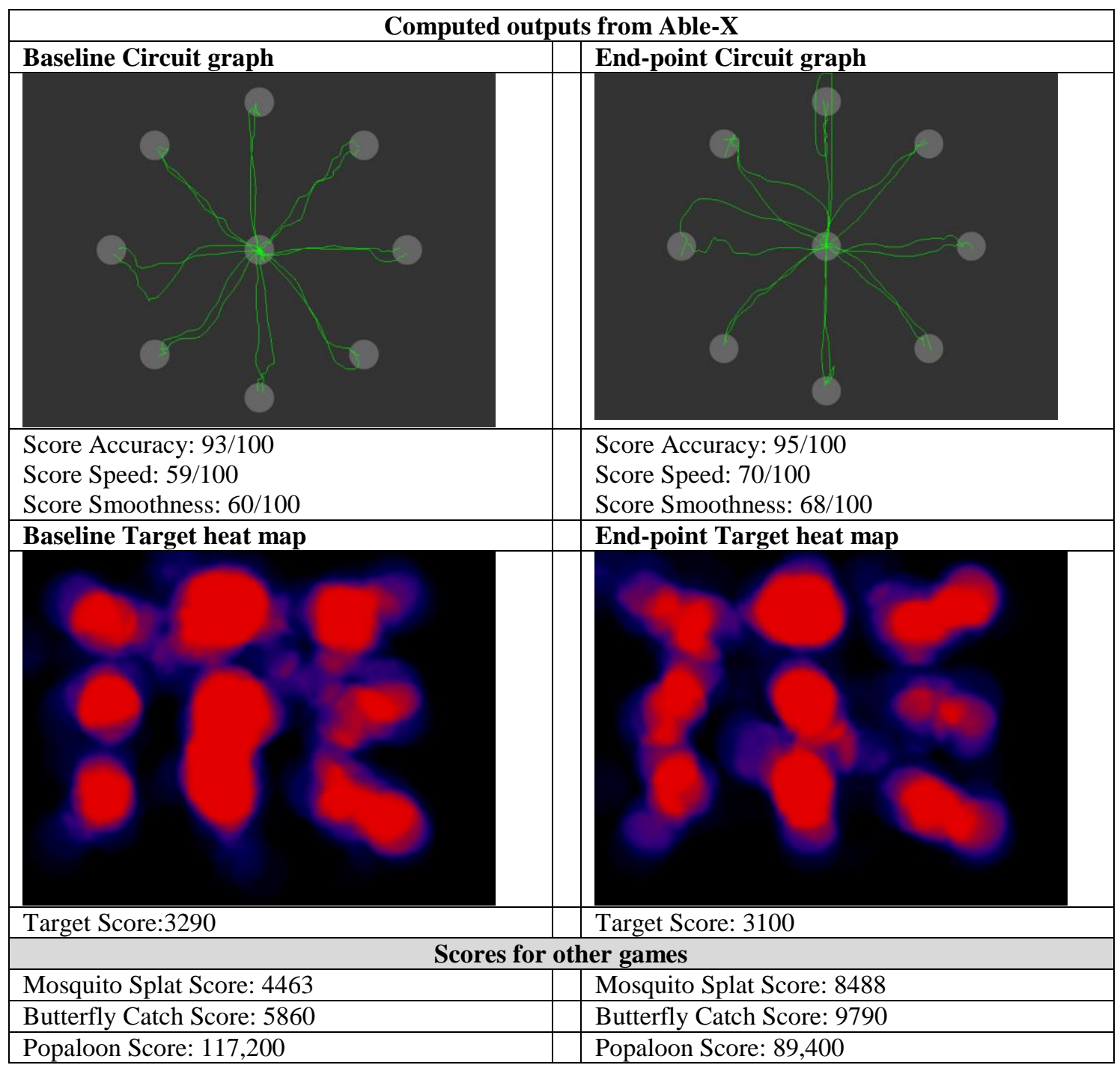

Figure 13. Risa: Computer-generated exergaming outputs 
There was a slight improvement by two points for accuracy, even though she had an excellent beginning score of 93/100. Improved smoothness of movement and speed to target scores indicates that she was getting to target quicker without reducing her accuracy scores. Conversely, reduced activity and score are evinced on the gaming outputs for Target. The Target heat maps show very slight reduction of on-screen activity and the Target score is reduced by 190 points. Risa's gaming was much less variable for the games of Mosquito Splat and Butterfly Catch, where her scores nearly doubled in each of these games at end-point. However, when non-target images appeared on-screen, verbal assistance was required, so there is no claim to improved visual discrimination in this case.

For the final game Popaloon, Risa achieved a reduced score of 27,800 points. However, in Risa's case, the reduced scores for Target and Popaloon could be explained by longer periods of independent gaming agency, which by the last session verbal assistance had reduced from every 5-10 seconds to one period of four minutes in one gaming sequence (Popaloon), therefore, although her score was reduced, improved cognition in terms of concentration and pattern recognition was evident.

\section{Comparing baseline measures and end-point measures}

Risa's cognitive MMSE 2-BV was unchanged at 7/16 at the end of gaming (Table 21). This MMSE score is unusually low for Stage 4 global decline (Reisberg, 1988; Auer $\&$ Reisberg, 1997). However, functionally, Risa continued to enjoy independent agency for ADLs and some IADLs, the RN testifies to this: "It's hard with [Risa] because the girls [HCAs] don't do much for her...they make her bed and make sure she's got everything she needs" (I:22/8:1349). The concern with this is they could be disempowering her IADLs agency to organise her own living space.

Table 21. Risa: Summary of baseline and end-point measures

\begin{tabular}{|l|r|r|}
\hline Validated measure & Baseline & End-point \\
\hline MMSE 2-BV & $7 / 16$ & $7 / 16$ \\
\hline RAID & $1 / 18$ & $1 / 18$ \\
\hline CSDD & $0 / 38$ & $1 / 38$ \\
\hline QOL-AD: Self-report & $47 / 52$ & $49 / 52$ \\
\hline QOL-AD: RN Proxy & $45 / 52$ & $47 / 52$ \\
\hline QOL-AD: NOK Proxy & $34 / 52$ & $40 / 52$ \\
\hline
\end{tabular}


The RN reported that Risa "is not being so bossy... as she was before” (I:11/8:831). "I've always seen her sort of like, hurrying the others up... and she holds on to the remote control [for the TV]" (RN:I:22/8:1375: I:11/8:831). This bossiness and controlling of the remote was seen as problematic, a negative change, documented in her clinical record by the RN following reports from the HCAs, who were not permitted to write in the health record. There was no change in the RAID anxiety score, which was very low at 1/18 on both occasions. Others reported "she's a little less uptight... a little less anxious (RN:I:11/8:842). The HCA stated, "[She is] a lot happier...none of this rush, rush [behaviour]" (RN:I:22/8:1393). The CSDD indicated a one-point increase to 1/38 at endpoint, in the aspect of irritability, and the RN indicated this was related to bossiness behaviour.

Overall QOL had improved by 10 points according to the joint ratings. The biggest rated change was from the NOK who awarded an extra six points at end-point. Risa and the RN reported a positive change, awarding an extra two points at the final assessment (Table 22). Both Risa and the RN, rated improved 'Physical health', the NOK rated no change in this item. Improved independent agency validated the extra point for 'Ability to do chores', awarded by both self and NOK. The RN thought this item was already excellent. The biggest change was three extra points awarded by the NOK for 'Ability to do things for fun', reporting "she was playing [the piano] for the ladies...I got a couple of pictures of her playing the piano... and they were singing” (NOK:I:23/8:1562). Both Risa and the RN rated no change in this item, rating it excellent on both assessments.

Table 22. Risa: Quality of life points change pre-and post-gaming

\begin{tabular}{|c|c|c|c|c|c|c|}
\hline Items on the QOL-AD questionnaire & $\begin{array}{l}\text { Self } \\
\text { B }^{*}\end{array}$ & $\begin{array}{l}\text { Self } \\
\mathbf{A}^{* * *} \\
\end{array}$ & $\begin{array}{l}\mathbf{R N} \\
\mathbf{B}^{*}\end{array}$ & $\begin{array}{l}\mathbf{R N} \\
\mathbf{A}^{* *} \\
\end{array}$ & $\begin{array}{l}\text { NOK } \\
\mathrm{B}^{*}\end{array}$ & $\begin{array}{l}\text { NOK } \\
\text { A }^{* *}\end{array}$ \\
\hline Physical health & 3 & 4 & 3 & 4 & 3 & 3 \\
\hline Energy & 3 & 3 & 3 & 4 & 4 & 3 \\
\hline Mood & 4 & 3 & 3 & 3 & 3 & 4 \\
\hline Living situation & 4 & 4 & 4 & 4 & 3 & 3 \\
\hline Memory & 3 & 3 & 2 & 3 & 1 & 1 \\
\hline Family & 3 & 4 & 4 & 3 & 4 & 3 \\
\hline Marriage (closest relationship) & 4 & 4 & 4 & 3 & 4 & 3 \\
\hline Friends & 4 & 4 & 4 & 4 & 1 & 3 \\
\hline Self as a whole & 4 & 4 & 4 & 4 & 3 & 4 \\
\hline Ability to do chores & 3 & 4 & 4 & 4 & 3 & 4 \\
\hline Ability to do things for fun & 4 & 4 & 4 & 4 & 1 & 4 \\
\hline Money (not relevant to gaming) & 4 & 4 & 3 & 3 & 1 & 1 \\
\hline Life as a whole & 4 & 4 & 3 & 4 & 3 & 4 \\
\hline Total Score & 47 & 49 & 45 & 47 & 34 & 40 \\
\hline Total QOL points change & & +2 & & +2 & & +6 \\
\hline
\end{tabular}

B*=Baseline score (1=poor, 2=fair, 3=good, 4=excellent). A**=After (end-point score). 
The NOK also awarded two extra points for the item 'Friends' (at the rest home), reporting that she had noted that Risa had become more social and engaged in activities. Risa reported this item as excellent and stated, "the friendships I make are always sound and happy" (R:I:22/8:1097). Risa rated her relationship with the item 'Family' had improved by one point, although her NOK and the $\mathrm{RN}$ rated this item as having deteriorated slightly. This score could reflect the NOK's guilt about her lack of presence: "I see her when I have a weekend off and that's not very often (NOK:I:23/8:1527. "She [Risa] always says we never visit or phone...we [siblings] treat it as a family joke" (NOK:I:26/7:797). In contrast, Risa declared, "I've got three children, I don't see a lot of them but we keep in touch and that is the main thing" (R:I:22/7:165). In items 'Self as a whole' and 'Life as a whole', all three raters agree that both these items were excellent at the final assessment.

\section{Case summary and postscript}

Risa had no conscious memory for gaming so every time we met it was introductory until she was actually playing and then she connected to a previous memory of gaming. In early gaming sessions, Risa needed near constant prompting, but towards the end of gaming, she could game, with independent gaming agency and less frequent prompting. Gaming with Able-X in partnership and at times independently was a psychosocial intervention that resulted in improved QOL outcomes for Risa. The most positive change effects were associated with improved independent intentional bodily agency that was initiated in the gaming room but extended back to the residence. Of particular note was how the splat and gore of the error screen connected Risa to wonderful memories of her life as a painter and the on-screen noises triggered memories of her life as a wedding singer and piano player, and rekindled her passion for playing the piano and singing. On occasions, this newfound agency was perceived as being bossy and controlling by staff. The other key finding was improved hand-eye coordination, concentration, and pattern recognition. There could be a link between these changes and her increased motivation to play the piano on a more regular basis during the gaming period.

Twelve months after the gaming experience, the $\mathrm{RN}$ reported that Risa's "shortterm memory is worsening and dementia is progressing but she continued to play the piano and sing several times a day [much more than pre-gaming] which is very entertaining for 
the others who love to attend her sessions" (RN:I:18/7:1698). This finding could be attributed to improved hand-eye coordination skills. Last word from Risa: "I enjoy life...I've got plenty of friends ...life is excellent”' (R:I:27/7:178, 196).

\subsubsection{Clara $^{30}$}

She's a strong woman who knows her own mind (RN:I:22/7:185). She was, interested in the world, independent, self-initiator...very interested in history...politics [and] social justice....Compared to how she was, her confidence is much lower, her memory loss is greater...I think her grip on reality is fading. (NOK:I:21/7:284, 282)

\section{Personal and clinical profile}

Clara was a slightly built, average height nonagenarian (91), female, born in England and emigrated to NZ in the 1950s. In 2011, at the age of 88, Clara had the first in a series of stokes ${ }^{31}$. Over the next three years, she became increasingly dependent on her two daughters as she lost IADLs ability:

We noticed her memory going and it just got more and more difficult for her to manage at home....We were paying bills, doing her shopping, writing her shopping list, doing her washing...we were doing everything for her. (NOK:I:21/7:294)

She had lived on her own for over 40 years but was now frightened at night, so her daughters took turns to stay over, "it was just a...complete pain in the ass, she...needed constant supervision" (NOK:I:21/7:328). In December 2013, she had another stroke. Her memory "took a big dive" (NOK:I:21/7:305). She was discharged from hospital to a residential home, but did not settle. Her daughters looked for an alternative residence and selected rest home accommodation at Gardenside. "It might only [provide]...dumb activities [but] at least there are people around for social engagement and the carers are good" (NOK:I:21/7:452).

\footnotetext{
${ }^{30}$ Pseudonym: Clara means "Clear, bright" ("http://www.thenamemeaning.com/clara/).

${ }^{31}$ Stroke: (left sided cerebrovascular accident (CVA).
} 
In April 2014, Clara, relocated to Gardenside, she was 91 years old. On admission, a consultant geriatrician assessed her and a diagnosis of $\mathrm{VaD}$ was made. Her NOK, requested that the word dementia was not used in Clara's company, the family refer to her condition as "short term memory problems... and have never used the word dementia in front of her" (NOK:I:18/7:3). The word dementia was edited out of all study documentation by the NOK, before it was shared with Clara (as discussed in section 3.4.5). Clara had been resident in Gardenside for three months when this study commenced.

I think she's happy now, she likes where she is which is a major turnaround from when we first visited...she said 'if you put me there I'll die (NOK:I:21/7:340). [Clara is] more accepting now that she needs to be here.... She said she 'felt tranquil and accepting of her situation. (RN:I:22/7:187, 233)

In July 2014, Clara enjoyed slow but steady ambulation with the support of a walking stick that helped steady her as she mobilised with the added challenge of vertigo. Although she had lost most IADLs abilities, she retained the ability to keep her room tidy:

I think she would have made her own bed until the day she died and that's from her navy days...Her bed was always immaculate. I noticed...they come in and make it, so she's sort of lost that, and they do the cleaning in her room. (NOK:I:21/7:762, 769)

Clara was fully independent with all basic ADLs, with gentle prompts. She functioned at Stage 4 on the FAST (Reisberg, 1988, 2007) and the GDS (Auer \& Reisberg, 1997), a stage where "Individuals generally have deficit in management of instrumental activities of daily life" (Auer \& Reisberg, 1997, p. 168) and has been equated to a clinical diagnosis of mild dementia (Reisberg, 2007). Clara was prescribed three regular medications, two of which had a sedative and mood-altering effect: lorazepam (anxiolytic) and citalopram (antidepressant) (Medsafe, 2015c; 2014e). 


\section{Engagement in gaming}

I'm glad to be able to help you coz it helps me as well. (C:I:22/7:1234)

Clara had 20 years of experience using a computer, albeit for communication (email). She lost the ability to use a computer in 2012, "that was one of the great shames of memory loss ... she just couldn't remember how to work it" (NOK:I:21/7:544). She needed assistance to connect with the computer-assisted exergaming technology, but once gaming she played with independent gaming agency from the very first session, with minimal prompting and assistance other than for the game Popaloon. She had a very nice playing posture (VL:20/8:20:00) and handled the Able-X controller well, incorporating the associated drift into her game without comment (VL:28/7:19:00). She tended to shuffle in towards the gaming screen, as her vision was impaired, but she did not wear corrective lenses, she used a hand-held magnifying glass for reading, but not for gaming. Clara played 9 out of 12 solo gaming sessions. She declined on two occasions when she was acutely unwell and on another occasion, she was unavailable due to an unexpected outing with a close friend. She played: Circuit $(n=9)$, Target $(n=9)$, Mosquito Splat ( $n=9)$, Butterfly Catch $(n=9)$ and Popaloon $(n=9)$.

Gaming with Clara was introductory, because at the start of every session she would be a little impeded in movement and speech, before becoming awakened by gaming. I would ask how she was feeling and she would describe herself in terms of her perceived level of consciousness and lucidity: "I feel semi-conscious" (VN:28/7:2236); "My brain is only half awake" (VN:29/7:2293); "I feel a little dopey, not fully awake" (VN:30/7:2349); “I feel half asleep" (VN:6/8:2429); “I don't know what I'm doing to make me feel so tired" (VN:12/8:2528). Conversely, after she had been gaming for about four minutes, sometimes longer, she would become more alert and very eloquently wakened: "this [gaming] makes me feel awake" (VN:28/7:2250); "The scores tell me I have good concentration [and] I have control of my brain" (VN:30/7:2359); "It is good [gaming] it stimulates something in the brain...It shows my brain is still active" (VN:5/8:2406); "[Gaming] has taxed my brain quite a bit...it feels good" (VN:5/8:2411); "Once you get into it you could play again and again...it certainly wakes the brain up" (VN:11/8:2514); “Every time I play I get a little quicker” (VN:12/8:2561); “It [gaming] certainly woke me up" (VN:13/8:2611); "I feel quicker" (VN:20/8:2673). 
During each gaming session, there was a clear continuum of cognitive agency from semi-wakefulness and need of assistance (VL:29/7:00:00) to awakened (04:40), to inquisitive (04:40), comparative (05:50), evaluative (10:04), game able (20:30) and alert and bright (33:00). Although she became more awake during gaming, wakefulness extended beyond the gaming room. Once awakened Clara was the most inquisitive of all the participants. She asked questions and sought information on how her game could be improved (VN:29/7:2294). The computer-generated scores and outputs were very important to her, as were the positive on-screen messages and other sources of encouragement that resulted in gaming confidence. "I am impressed with myself" (VN:28/7:2253). I asked her if the games were too easy and she said, "they were just about right" (VN:12/82541). She continued to enjoy the repeated success of gaming at the same level.

Gaming with Clara was punctuated by moments of quiet conversation, usually relating to the game in hand, or periods of quiet concentration and focus. She was, "quite an intellectual” (NOK:I:8/8:1369), [and] “quite a serious lady” (RN:I:22/7:200), who needed "a start of the day event to activate her day" (NOK:I:21/7:26). Gaming with Able$\mathrm{X}$ appeared to be a very appropriate way to activate the day. Clara always left the gaming room alert, bright, and chatty and this demeanour was maintained beyond the gaming room. "I think there has been a big improvement [She is] coming to more activities and engaging more. The fact that she's actually...going looking for activities rather than us having to come to her [is evidence of change]" (RN:I:22/8:1690, 1699, 1710).

Two gaming situations would trigger disengagement from gaming with Able-X. Negative gaming noises caused a startle effect that momentarily disrupted her gaming and appeared to trigger a run of errors or momentary gaming inertia (VL:13/8:07:00, 09:50), following which she would require re-initiation to become gaming active again. She also had difficulty with colour differentiation between blue, purple, red and green on-screen images, and again needed verbal and pointing assistance to progress. However, unlike some participants, Clara did not appear to be unduly hampered by the drift associated with the Able-X controller; she was able to adapt her play to accommodate it, or graciously accept assistive touch without comment. 


\section{Gaming outputs}

Clara's gaming scores were relatively stable during the gaming period, gaining small improvement in two aspects of the Circuit assessment and a slightly improved score for all the other games (Figure 14):

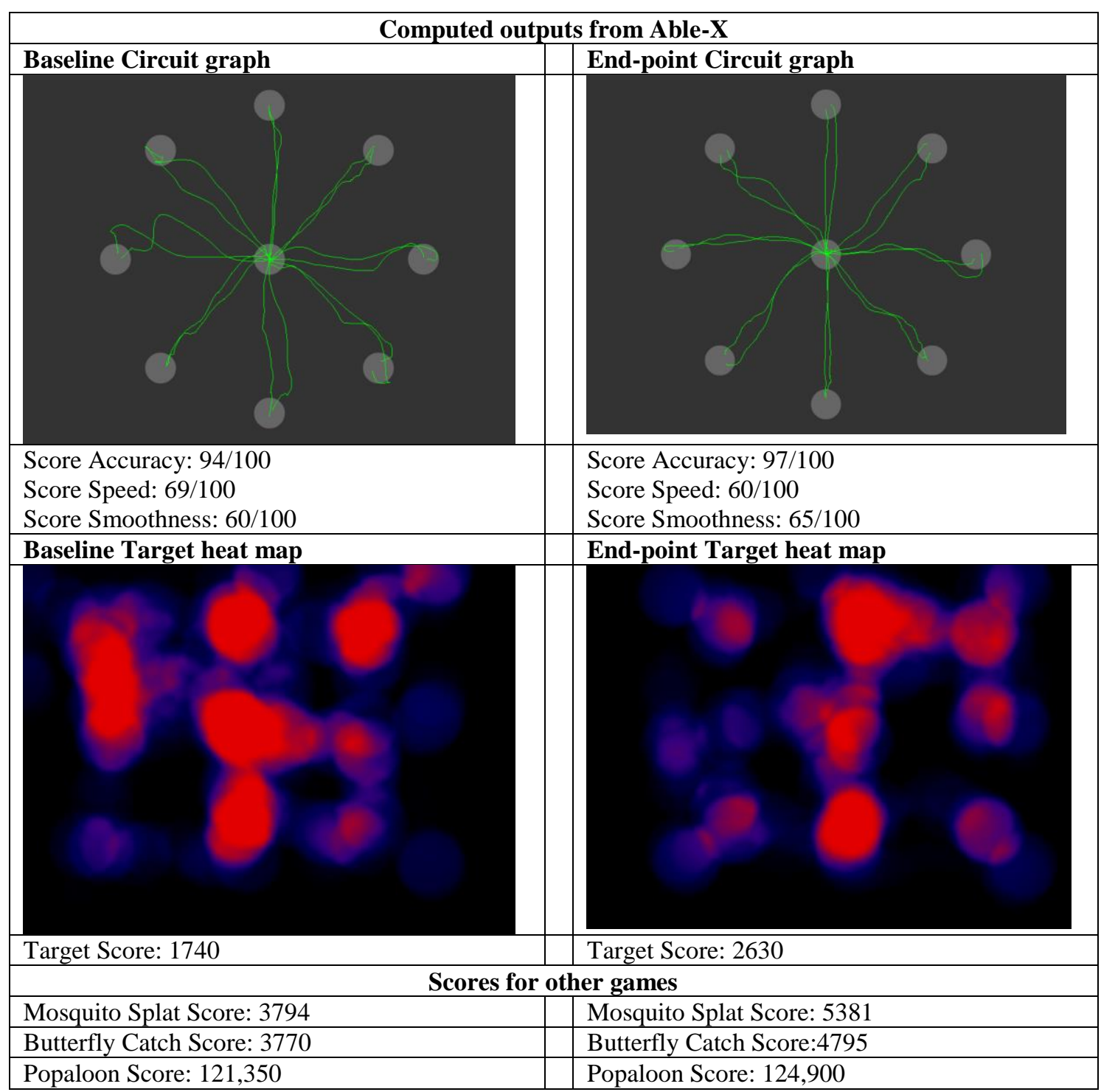

Figure 14. Clara: Computer-generated exergaming outputs

Clara's beginning score for accuracy of movement was an excellent score of 94/100 at baseline, leaving not much room for improvement; however, she did improve by three points to 97/100. Also improved at end-point was smoothness of movement by five points to $65 / 100$. These improvements impacted on gaming speed which reduced by nine points to $60 / 100$ at final assessment. Improved Target score infers slightly improved hand-eye coordination; however, the Target heat map-activity graph appears to show slightly less 
activity overall, other than the in the central field, therefore caution is required when implying improved hand-eye coordination from this game.

Clara also had improved scores for the games Mosquito Splat, Butterfly Catch, and Popaloon. However, she needed verbal assistance to avoid non-target on-screen images, more so for Mosquito Splat, so there is no claim of improved visual discrimination in this case. In addition, although her score improved for Popaloon, she had difficulty seeing some of the coloured images (green and blue), and needed verbal prompting and screen pointing assistance. That said, for the colours she could see, she demonstrated good concentration and pattern recognition skills.

\section{Comparing baseline measures and end-point measures}

Clara's cognitive MMSE 2-BV score deteriorated at end-point by three points, all related to orientation to time and place (Table 23). As her NOK reiterated, her memory was in decline before the study commenced. Clara also had episodes of acute head pain towards the end of the gaming period that could be connected to the low MMSE score.

Table 23. Clara: Summary of baseline and end-point measures

\begin{tabular}{|l|r|r|}
\hline Validated measure & Baseline & End-point \\
\hline MMSE 2-BV & $9 / 16$ & $6 / 16$ \\
\hline RAID & $8 / 18$ & $0 / 18$ \\
\hline CSDD & $3 / 38$ & $0 / 38$ \\
\hline QOL-AD: Self-report & $38 / 52$ & $40 / 52$ \\
\hline QOL-AD: RN Proxy & $40 / 52$ & $47 / 52$ \\
\hline QOL-AD: NOK Proxy & $25 / 52$ & $37 / 52$ \\
\hline
\end{tabular}

This MMSE 2-BV score is unusually low for Stage 4 global decline (Auer \& Reisberg, 1997; Reisberg, 1988). The MMSE results were not surprising to Clara's NOK: "It might not have had an effect on her memory...but it has had multiple other effects [it] helped create a sense of connection...she felt engaged" (NOK:I:27/8:2012). The RN reported, "she doesn't need to be prompted to go to meals and stuff like that [since gaming]” (RN:I:22/8:1722).

Functionally, Clara was independent in basic ADLs with only minimal supervision, and following gaming with Able-X, she appeared awakened, alert and bright, which afforded her opportunities of independent agency to be social and engaged within 
her environment. Improved wakefulness and lucidity is evidence of improvement in BPSD by association $\left(\mathrm{Bpac}^{\mathrm{nz}}, 2013\right)$. The $\mathrm{RN}$ reported, she is smiling and interacting with other residents (RN:I:22/8:1692). Both the RN and NOK awarded extra QOL points for the item of 'Energy' at the end-point, which could be reflective of the intra and post gaming wakefulness. There was also evidence of improved cognition in respect to concentration and pattern recognition, as demonstrated by improved gaming scores on Popaloon (Able$X, 2013)$.

At the beginning of the study, Clara was showing signs of anxiety with a RAID score of $8 / 18$, by the end-point, her score was zero. There were also other indications of changed anxiety levels: "she is an anxious person but we haven't seen any anxiety especially over the last couple of weeks I think she's a lot calmer.... We haven't had any of the, oh I'm going to my daughters...I've got to pack" (RN:I:22/8:1714 \& 1734). An improved depression score was also evident at the final assessment, with the score of zero points (CSDD). Further evidence of improved mood includes, "[she has]...been more accepting of the situation of where she is.... and understands that if she was in her own home ... she wouldn't be able to function and manage properly (NOK:I:24/8:1808). She is also "a lot happier" (RN:I:22/8:1688) and clearly taking an interest in what is going on around her as she engages in activities within the residence. The RN and NOK awarded an extra point for 'Ability to do things for fun', the NOK stated, 'I think what you did for her...that month probably helped propel her ...to engagement" (I:26/8:2064), (Table 24).

Table 24. Clara: Quality of life points change pre-and post-gaming

\begin{tabular}{|c|c|c|c|c|c|c|}
\hline Items on the QOL-AD questionnaire & $\begin{array}{l}\text { Self } \\
\text { B }^{*}\end{array}$ & $\begin{array}{l}\text { Self } \\
A^{* * *}\end{array}$ & $\begin{array}{l}\mathbf{R N} \\
\mathbf{B}^{*}\end{array}$ & $\begin{array}{l}\mathbf{R N} \\
\mathbf{A}^{* * *}\end{array}$ & $\begin{array}{l}\text { NOK } \\
\text { B* }^{*}\end{array}$ & $\begin{array}{l}\text { NOK } \\
A^{* *}\end{array}$ \\
\hline Physical health & 3 & 4 & 3 & 3 & 3 & 3 \\
\hline Energy & 3 & 3 & 2 & 4 & 1 & 2 \\
\hline Mood & 3 & 3 & 3 & 4 & 3 & 4 \\
\hline Living situation & 3 & 3 & 4 & 4 & 3 & 4 \\
\hline Memory & 2 & 3 & 2 & 3 & 1 & 1 \\
\hline Family & 2 & 3 & 3 & 3 & 1 & 1 \\
\hline Marriage (closest relationship) & 3 & 3 & 3 & 3 & 3 & 3 \\
\hline Friends & 1 & 3 & 3 & 4 & 1 & 4 \\
\hline Self as a whole & 2 & 3 & 3 & 4 & 1 & 4 \\
\hline Ability to do chores & 3 & 3 & 4 & 4 & 1 & 3 \\
\hline Ability to do things for fun & 3 & 2 & 3 & 4 & 3 & 4 \\
\hline Money (not relevant to gaming) & 4 & 4 & 3 & 3 & 1 & 1 \\
\hline Life as a whole & 2 & 3 & 4 & 4 & 3 & 4 \\
\hline Total Score & 34 & 40 & 40 & 47 & 25 & 38 \\
\hline Total QOL points change & & +8 & & +7 & & +13 \\
\hline
\end{tabular}


The RN and NOK awarded an extra point for the item 'Mood', the NOK also awarded an extra point for 'Living situation', reflecting that Clara had settled in place. Improvement in levels of anxiety level and depression demonstrates improvement in BPSD (Bpac $\left.{ }^{\mathrm{nz}}, 2013\right)$, which is an indicator of QOL in PWD (Prince et al., 2014). It is likely in this case that medication for anxiety and depression may have contributed to changes in anxiety levels and mood and certainly to her level of drowsiness, as both have sedative effects.

Overall QOL had improved by 28 points across all raters. All agreed that the item of 'Friends' had improved, and the NOK thought that this item had improved by three points, adding "I think that she's made excellent friends with [name] across the corridor" (NOK:I:24/8:1891). Clara agreed that this was the most improved item. The RN testified, "[Clara is now] smiling...interacting...[and] more social" (I:22/8:1692). All were in agreement that the item 'Self as a whole' improved by at least one point, and according to the NOK by three points, making this item good according to Clara and excellent according to the RN and her NOK who had scored this item poor at baseline. The item 'Life as a whole' also improved according to Clara and her NOK, rating this item good or excellent, also proposing:

Given everything, I think [her QOL] it's actually excellent, but that's mainly to do with her lift in engagement...If she hadn't had that lift...there would still be problems (NOK:I:27/8:2131). I would agree...whole heartedly...that her...wellbeing and her demeanour and her...intrinsic happiness is markedly improved....That would be where the biggest change is and the fact that she is very settled, and very happy and I'm quite sure that... [exergaming with Able-X] played quite a big part. (NOK:I:27/8:1998)

\section{Case summary and postscript}

Clara had no conscious memory of gaming so every time we met was introductory until she was playing and then she connected to previous memory of gaming. From the first gaming session, she could follow instructions and quickly moved to independent gaming agency as she became more confident. Clara was distracted by negative on-screen messages and noises, which at times interrupted her independent gaming agency and resulted in a run of errors, which would continue until she regained independent gaming 
agency. She was also distracted by her inability to discriminate between some of the colours on the gaming screen due to her impaired vision.

Gaming with Able-X resulted in improved QOL outcomes for Clara. The most positive change effects were associated with improved wakefulness that allowed her to become increasingly successful at gaming, and enabled her to reconnect to her inquisitive, competitive, evaluative, and wakeful self. Once connected to wakefulness, she could extend independent agency beyond the gaming room and back into the residence, where she purposefully engaged herself socially and recreationally, demonstrating improvement in the BPSD associated with lucidity and wakefulness $\left(\mathrm{Bpac}^{\mathrm{nz}}, 2013\right)$. The computergenerated outputs demonstrated improved hand-eye coordination, concentration, and pattern recognition. What was exciting about Clara's case was the revelation of concepts of intra and post-game wakefulness, the potential of day activation events, and how negative error messages linked to a run of errors and gaming inertia.

All the improvements in wakefulness, connection to social self and hand-eye coordination were maintained at 12 months post gaming. The $\mathrm{RN}$ reported that in order to capitalise on the gains made through gaming with Able-X, wakefulness was initiated and maintained through the introduction of a 'start of the day event'. "She likes the cat and the cat visits her room every morning...She was given the responsibility for feeding the cat and the fish....She has also been allocated a garden space [she gets up every morning to do both]" (RN:I:18/7:2199). Her improved hand-eye coordination has translated to "more independence with washing and showering... She is also cleaning her room ... [and] playing bowls (RN:I:18/7:2212). Because Clara is more awake she can "get from A to B without forgetting what she is doing” (RN:I:18/7:2205), so she is more social and active. Finally, she is no longer "constantly looking to go home" (RN:I:18/7:2219). Last word from Clara: "I forget I'm 91 sometimes. I'm supposed to be full of arthritis with a walking stick [laughs]" (I:30/7:2352). 


\subsubsection{Alfie A2 $^{32}$}

He's lovely...a wonderful guy...very popular....He's...young and he's very uncomplaining, civilised, and loves the banter...a cheeky chappie. (RN:I:10/9:517)

\section{Personal and clinical profile}

Alfie was heavier than average for weight and height. A sexagenarian male who, dreams about "getting out of his wheelchair...walking...and playing tennis" (FN:15/9:1535). He was a divorced father of four children, who "don't seem to want to come and see him" (NOK:I:15/9:190). The estrangement occurred years ago when Alfie "caused a lot of trouble trying to get custody [of his children]" (NOK:I:15/9:194). His closest relative and NOK was one of his three older brothers, who was also EPA for Personal Care and Welfare.

In 2008 at the age of 56, Alfie had what would be the first in a series of four strokes that caused left sided hemiplegia, requiring continued hospital level care in the care home sector. In 2009, a second stroke left him with short-term memory problems and expressive dysphasia, "he finds it very hard to talk [and]...he will start crying" (NOK:I:15/9:121). When he did talk, his speech was slightly stunted and slurred. Sometimes he did not speak, but used gestures such as smiles, thumbs up, nod or point, and interjections such as, "aah, ooh, mm, aw, hmm" (VN:10/9:416). He was prescribed regular diazepam (benzodiazepine) used to treat agitation. Benzodiazepines are known to cause confusion, impaired cognition, and sedation $\left(\mathrm{Bpac}^{\mathrm{nz}}, 2008\right)$, and this could have attributed to his lagging interaction and slurred speech.

Alfie's last stroke in 2011, "was a bad one... he nearly died. They...tried to get me to sign papers to say not to resuscitate him...I said 'no...you're the doctors, you'll do your best'” (NOK:I:15/9:94). Following this event, he was diagnosed with VaD by his GP. The following year (2012), a decision was made to relocate him to Lakelands for continued hospital level of care, in a location distant from his hometown but closer to his three

\footnotetext{
${ }^{32}$ Pseudonym: Alfie means "Ready for battle" (http://www.thenamemeaning.com/alfie/).
} 
brothers and only visitors. One brother visited once a week, the other two "go and see him when they can" (NOK:I:15/9:187). Alfie was 60 years old at the time of his admission, and had been at Lakelands for two years when this study commenced. "He wants to get out... and I keep telling him, you get that left arm and...leg moving, come out the door and I'll be waiting for you (NOK:I:15/9:143).

In July 2014, Alfie was wheelchair bound and fully dependent on others for all ADLs, other than feeding himself, which was supervised. There were some behaviour issues around toileting that created tension between himself and his caregivers. Alfie often had coughing fits due to post stroke dysphagia. However, Alfie had retained some IADL ability for example; he could work the remote control to access the television and his music centre, both of which he enjoyed. It was too dubious to assess Alfie's functional assessment and global deterioration using the FAST (Reisberg, 1988) and the GDS (Auer \& Reisberg, 1997) because it was difficult to determine which aspects of disability, loss of functioning and decline were related to his multiple strokes and which were related to $\mathrm{VaD}$, so they were not used in this case. Alfie's baseline MMSE-2:BV (Folstein et al., 2010) was $4 / 16$ and has been equated to a clinical diagnosis of severe dementia (Reisberg, 2007).

\section{Engagement in gaming}

Alfie was computer literate and had played computer games before, so was eager to engage in gaming with Able-X. He played 11 out of 12 solo gaming sessions, including Circuit $(n=13)$, Target $(n=13)$, Mosquito Splat $(n=11)$, Butterfly Catch $(n=11)$ and Popaloon $(n=11)$. Alfie declined to game on one occasion due to an acute illness, and on three occasions gaming was delayed, because his caregivers were late getting him up for the day. These delays were a known irritation for Alfie, "there's a little bit of frustration ... but we have 32 [patients] to prioritise" (RN:I:2/10:430).

After the third gaming session, staff reported, that he recalled information about gaming when asked (RN:I:2/10:452). By the tenth gaming session, Alfie could select game choices and levels with absolute independent gaming agency. He knew the start-up routine and assisted with game preparation. He was the only participant to achieve this level of gaming independence. 
Alfie was a quiet but confident gamer, a man of few words who nodded or pointed at the screen rather than talking (VL:10/9:00:00). An offer to make the games more challenging was declined, as he preferred the repeated success of easy gaming and simple time-limited games. Alfie played two extra Circuit and Target sessions, this was because he had played with both hands and arms, utilising the Able-X handle bar (VL:12/9:03:00), which was developed specifically for stroke survivors or others with neurological impairment (Im-Able Ltd., 2013). However, he was disappointed with the results from his two-handed efforts (VL:12/9:03:00; VL:22/9:03:50) so he repeated each game with his dominant uninjured hand and arm.

The contemporaneous scores and positive on-screen messages were important to Alfie. He enjoyed early success and I told him such good results would be hard to equal or surpass and he said, "I like a challenge" (VN:10/9:1434). He also enjoyed inspecting his results, scores, and positive messages both during and at the end of each game (LV:25/9:09:00). Alfie was very self-competitive and his scores were dependent on his level of wakefulness, which was variable, especially at the beginning of gaming when he sometimes appeared a little impeded, especially on the days when I was waiting for him to be mobilised from his bed. However, once awakened, his wakefulness extended beyond the gaming room. "[He is] more alert, more on the page, more conversational. [He has changed in] lots of ways really. He can handle normal conversation really well [since gaming], but I think that comes through keeping him stimulated" (HCA:I:1/10:823).

Alfie rarely became disengaged from gaming once activated. If he did, it was usually the drift associated with the controller that he could not re-centre himself, due to having use of only one hand. By the sixth gaming session, he intuitively absorbed the drift into his gaming technique, and assistance from me was reduced.

\section{Gaming outputs}

Alfie achieved improved scores in all but one game. Circuit showed variable but improved scores for accuracy, smoothness of movement and speed to target. It is difficult to improve on a baseline accuracy of $95 / 100$, but Alfie did and his end-point accuracy rate was 97/100. Although Alfie's speed to target improved by 13 points, this improvement was based on a very slow baseline speed score, the improved speed did not appear to have a detrimental effect on accuracy or smoothness of movement, the latter of which slightly 
improved by 6 points at end-point assessment. As can be seen from the Target heatactivity map (Figure 15) Alfie also increased his activity, particularly around the peripheral gaming field. Both Circuit and Target outputs are suggestive of improved hand-eye coordination.

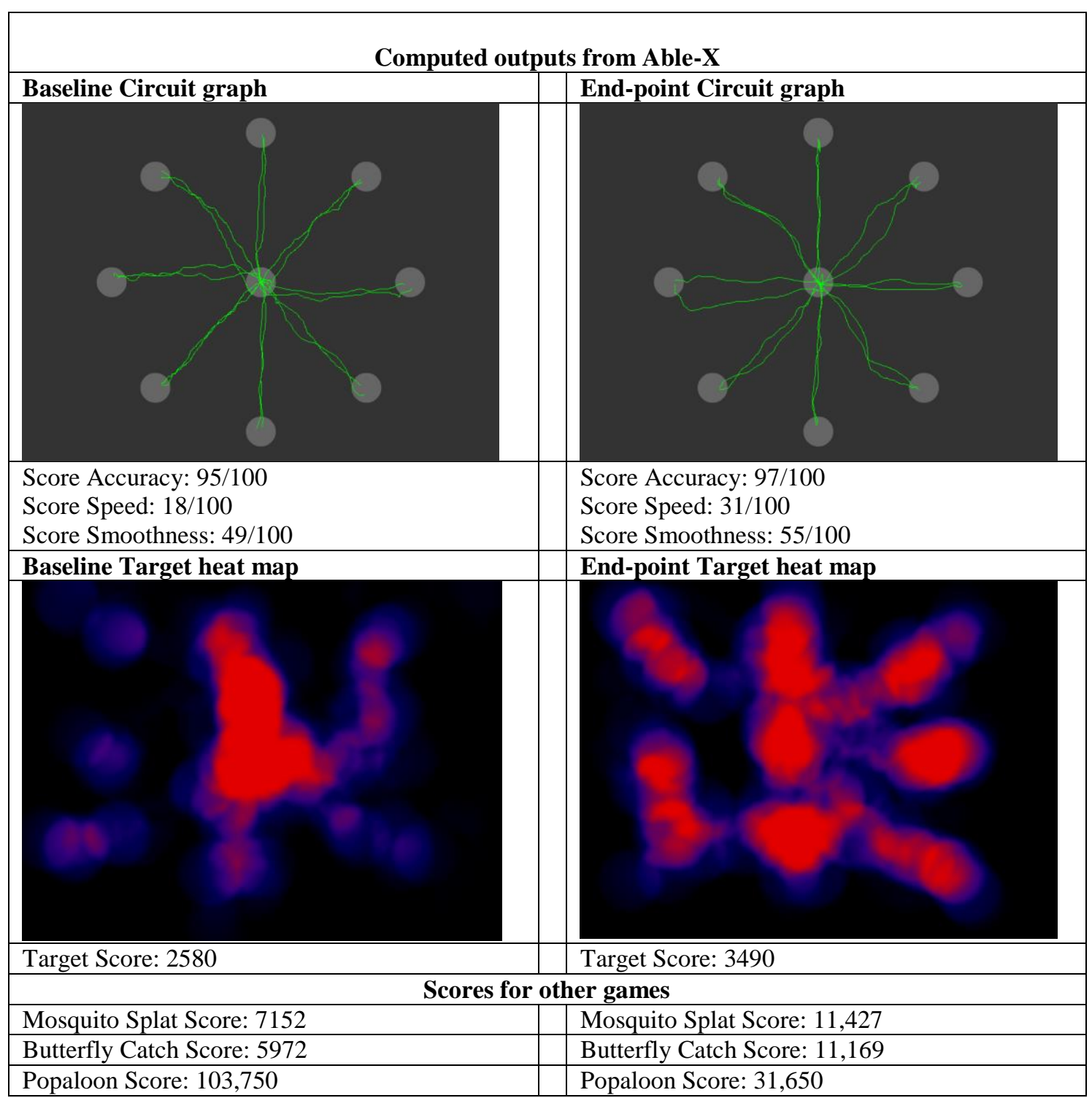

Figure 15. Alfie: Computer-generated exergaming outputs

There was more room for improvement in Mosquito Splat and Butterfly Catch, which builds on the primary skills of hand-eye coordination and introduces the challenges of visual discrimination. Improved scores of 4275 for the former and 5197 for the latter inferred improved hand-eye coordination and improved visual discrimination in this case. In the final game Popaloon, Alfie did less well according to the score. Although Alfie's score reduced on Popaloon by 72,100, he played the game unassisted and demonstrated 
the skills of concentration and pattern recognition. His slow and accurate gaming style did not lend itself to this game in which gamers were penalised if slow to target. Alfie was a slow but accurate and independent gamer.

\section{Comparing baseline measures and end-point measures}

Alfie's cognitive MMSE 2-BV score improved by three points at final assessment, with extra points being gained for orientation to time, place, and recall (Table 25). There was other evidence of improved cognition in respect to concentration, visual discrimination and pattern recognition, as demonstrated by gaming scores on Popaloon (Able-X, 2013). The RN reported, “I've noticed he's as stable as I think I've ever met him" (I:2/10:292). He was managing to maintain eye contact, which improved communication and connection with others:

Even his eye contact...[was] more solid...I don't know why it is, but he seems to have more control [of his eyes]...he seems to be able to focus much quicker after [gaming, and for] longer....No wandering, no looking at anyone else. He talked back, which itself is quite unusual because we usually get two or three words from him ...but he was chatting away. (RN:I:2/10:350)

Table 25. Alfie: Summary of baseline and end-point measures

\begin{tabular}{|l|r|r|}
\hline Validated measure & Baseline & End-point \\
\hline MMSE 2-BV & $4 / 16$ & $7 / 16$ \\
\hline RAID & $1 / 18$ & $0 / 18$ \\
\hline CSDD & $1 / 38$ & $0 / 38$ \\
\hline QOL-AD: Self-report & $45 / 52$ & $46 / 52$ \\
\hline QOL-AD: RN Proxy & $36 / 52$ & $40 / 52$ \\
\hline QOL-AD: NOK Proxy & $36 / 52$ & $35 / 52$ \\
\hline
\end{tabular}

Alfie was awakened through the process of computer-assisted exergaming with Able-X and this wakefulness extended beyond the gaming room, although for Alfie, his opportunities to enjoy wakefulness were dependent on others to transport him to less solitary situations, where he could take advantage of socialising and engaging in conversation and activities. The DT stated, "It would be good if they could get him up in the morning....He likes the paper and the quizzes...but I rarely see him. It makes me aware that we should be doing more stimulating activities with people like him” (1/10:931, 1008). 
Improvement in levels of anxiety level and depression demonstrate improvement in BPSD by association (Bpac ${ }^{\mathrm{nz}}$, 2013). At the beginning of the study, Alfie's RAID score for anxiety in dementia was $1 / 18$, the one point being for fatigability and tiredness. By the end-point, his score was 0/18. Similar scores were given for fatigue and lack of energy on the CSDD. Over the gaming period, computer-assisted exergaming with Able-X was the event that activated Alfie's day for three days a week for four weeks and this could have contributed to a reduced fatigue state. "He used to have a go [complain and grumble] at some of the caregivers...but not recently [since gaming], he's been wonderful....He's got all the patience in the world at the moment" (RN:I:2/10:303, 433). Although having less to complain about can infer improvement, in this case, this finding was a conundrum, as for Alfie, it was his independent agency to "have a go at some of the caregivers" (RN:I:2/10:303), that was the trigger event that activated his day and transported him from his bed and his small room to a more stimulating and social environment.

Overall QOL improved slightly according to the perceptions of Alfie by one point and the RN by four points. However, the NOK perceived a slight drop of one point (Table 26):

Table 26. Alfie: Quality of life points change pre-and post-gaming

\begin{tabular}{|c|c|c|c|c|c|c|}
\hline Items on the QOL-AD questionnaire & $\begin{array}{l}\text { Self } \\
\text { B }^{*}\end{array}$ & $\begin{array}{l}\text { Self } \\
\mathbf{A}^{* *} \\
\end{array}$ & $\begin{array}{l}\mathbf{R N} \\
\mathbf{B}^{*} \\
\end{array}$ & $\begin{array}{l}\mathbf{R N} \\
\mathbf{A}^{* *} \\
\end{array}$ & $\begin{array}{l}\text { NOK } \\
\text { B* }^{*}\end{array}$ & $\begin{array}{l}\text { NOK } \\
\text { A }^{* *}\end{array}$ \\
\hline Physical health & 3 & 3 & 2 & 2 & 3 & 3 \\
\hline Energy & 3 & 4 & 3 & 3 & 3 & 1 \\
\hline Mood & 3 & 3 & 3 & 4 & 3 & 2 \\
\hline Living situation & 4 & 4 & 3 & 4 & 4 & 4 \\
\hline Memory & 4 & 4 & 3 & 2 & 1 & 1 \\
\hline Family & 4 & 4 & 2 & 2 & 3 & 4 \\
\hline Marriage (closest relationship) & 4 & 4 & 2 & 2 & 3 & 4 \\
\hline Friends & 4 & 4 & 3 & 3 & 3 & 3 \\
\hline Self as a whole & 3 & 3 & 3 & 4 & 4 & 2 \\
\hline Ability to do chores & 4 & 4 & 3 & 3 & 1 & 4 \\
\hline Ability to do things for fun & 3 & 3 & 4 & 4 & 3 & 4 \\
\hline Money (not relevant to gaming) & 3 & 3 & 2 & 3 & 1 & 1 \\
\hline Life as a whole & 3 & 3 & 3 & 4 & 4 & 2 \\
\hline Total Score & 45 & 46 & 36 & 40 & 36 & 35 \\
\hline Total QOL points change & & +1 & & +4 & & -1 \\
\hline
\end{tabular}

$\mathrm{B}^{*}=$ Baseline score (1=poor, 2=fair, 3=good, 4=excellent). $\mathrm{A}^{* *}=$ After (end-point score).

Alfie self-rated all items of QOL-AD to be good or excellent at each assessment point and the RN concurred with Alfie's ratings on most items other than 'Physical health', 'Closest relationship', and 'Family', which the RN rated to be fair at baseline and no change at end- 
point. Alfie had lost contact with all friends and family other than with his three brothers, one in particular, who was his most frequent weekend visitor. This could explain the difference in ratings for these two items. The highest point change was rated by the NOK for the item 'Ability to do chores', for which he awarded three extra points. All raters agreed that at the end-point assessment Alfie had good to excellent ability to do chores within his level of capability and functionality, which included feeding himself and managing the in-reach environment.

The only item Alfie rated as improved was 'Energy' awarding one extra point for this item. Conversely, his NOK thought this item was lower by two points at the end-point assessment, describing Alfie as "a hemiplegic person who's dependent on others to... move around" (NOK:I:15/9:172). The inference being that Alfie is doing less for himself. Conversely, there was evidence that Alfie had begun to push boundaries to be more actively engaged in activities that he enjoyed. Usually when he returned from being out in the fresh air, he is exhausted and wants to go to bed, but during the gaming period he would say, "put me in there [social room], I'm gonna carry on"(RN:I:2/10:492). Alfie's improved energy and cognition levels could also explain improved ability to communicate and converse, "he was good...at story-telling...but there's tons more of that [since gaming]. To be honest, it might be not as much the game but also yourself, you've brought a lot of that out in him" (RN:I:I2/10:500).

The RN rated the item of 'Mood' had improved slightly by one point, adding, "at the moment he is...very happy...[with] less periods of confusion" (I:2/10:452). Conversely, the NOK did not agree and awarding minus one point for this item and stated, "He wants to get out of where he is [Lakelands]" (NOK:I:15/9:143). For the items 'Self as a whole' and 'Life as a whole', Alfie reported them to be good at each assessment point. The RN thought at end-point that both items had improved and were excellent, again the NOK assessed differently, and awarded two less points for each of these items at the final assessment and reported that every time he goes to see him "usually just before lunch, or just before dinner...he just sleeps" (NOK:I:6/10:38). The NOK only sees Alfie for an hour a week on a Sunday, when there is less activity going on in the residential setting, which could account for his ratings. Responding to the item 'Ability to do things for fun', the NOK agreed that this item had improved. Moreover, gaming with Able-X appeared to activate other fun situations: 
[He has] the look on his face that he's awake...I think this is the biggest thing looking from the outside in....His engagement with other people is another sign too...he's more able to engage... and he'll be bothered to try (HCA:I:1/10:845). I've seen a distinctive change in his confidence. I mean that conversation on Monday, he was focused, no mistakes in speech, so grade that as you will. (RN:I:2/10:508)

\section{Case summary and postscript}

Gaming with Able-X resulted in improved QOL outcomes for Alfie. Alfie found gaming particularly pleasurable. The computer-generated outputs demonstrated improved hand-eye coordination, visual discrimination, concentration, and pattern recognition. He was the only participant to achieve absolute independent gaming agency. The most positive change effects were those associated with improved wakefulness that extended beyond the gaming room and were evidenced by improved ability to engage in and sustain meaningful conversations and eye contact. Once connected to wakefulness, Alfie could extend himself through improved mood and communication with his caregivers and others. Furthermore, improved communication led to improved relationships and improved behaviour regarding toileting issues, all of which evince improved BPSD ( $\mathrm{Bpac}^{\mathrm{nz}}, 2013$ ). The conundrum elicited by Alfie's case was the realisation that prior to gaming, Alfie used independent agency to complain to caregivers until they mobilised him for the day. Throughout the gaming period, Alfie became more patient and tolerant. Alfie's case highlights the importance of an 'event to activate the day'.

Ten months post gaming Alfie continued to enjoy longer periods of wakefulness, improved eye contact, sustained meaningful conversations and purposeful engagement in all activities, which the RN equated with continued improved hand-eye coordination and concentration. Following my research, changes were made to Alfie's daily routine in an effort to activate his day and maintain the improvements brought about through gaming:

His behaviour is more appropriate...[he] assertively asks for toileting [less disruptive around toileting, which previously caused relationship problems with care staff]....He is now sleeping better...more wakeful and less drowsy....His days are more active...[He] takes part in all activities now....He is more wakefully...interactively engaged, [and] less passively engaged in activities. He 
has now got something to say and he says it out loud. His verbal communication [continues to be] improved [he was quiet and not very verbal pre-gaming]. He even asked that we decorate his room and make it more like a man cave, which we did. (RN:I:5/7:1283, 1319, 1333, 1349)

The NOK made no changes to the case transcript. Last words from Alfie: "It [Gaming] makes me feel good...[the feeling lasts] all day...Nothing else does this for me, I don't get the same feeling" (I:1/10:943).

\subsubsection{Alana A3 $^{33}$}

She is loving and caring, one of life's givers. She's got a lot of energy for life (FN:NOK:11/9). She smiles a lot...[and] she loves to touch [and] embrace, she's very tactile.... She has a wonderful facial expression, which tends to give us the idea she's in a really good space [or not]. (RN:I:9/9:490)

\section{Personal and clinical profile}

Alana was a slightly built, average height octogenarian (86) female, born in the Middle East. She emigrated to NZ in the early 1960s, with her Kiwi husband and youngest sister. Her mother died when she was 10 years old, and her father died three years later. As the oldest female in the family, she became homemaker for her two older brothers and child caregiver for her youngest sister. Her youngest daughter, who she describes as "the baby of my children ...my little angel” (A:I:9/9:256), was her NOK and EPA for Personal Care and Welfare.

In 2010, at the age of 82 , four years after she was widowed, Alana relocated from her marital home to live in a retirement village. Over the next three years, she became less able to cope in her independent living environment and her family noticed she kept leaving the village and getting lost. An assessment by the GP resulted in a diagnosis of dementia, likely Alzheimer's disease, for which she was prescribed donepezil and regular citalopram for depression.

\footnotetext{
${ }^{33}$ Pseudonym: Alana means "peaceful, serene" (http://www.thenamemeaning.com/alana/ ).
} 
In July 2014, at age 86, Lakelands became Alana's new place of residence in rest home level of care. It is distant from her friends in the retirement village, but nearer to her daughter. Alana had been resident in Lakelands for just over two months when this study commenced. She described her life as "very good...I'm having an easy time in my life. I would say [my living situation]...is on the excellent side. I don't get any problems here....I am very happy (A:I:9/9:87).

In September 2014, Alana enjoyed agile ambulation and mobilisation without aids, and with a little prompting. She had the physical benefits of someone who "walks a lot and it's been her pattern in life" (NOK:I:6/10:1418). She looked fit, lean and appeared full of energy. However, there was evidence of loss of some functioning, for example, Alana had lost all IADLs over the previous three years, she could no longer use the telephone, computer, or the remote controls for the television and music centre. Her NOK reported, "she's used to doing things with her hands all the time, and that seems to have slipped away since she's been in [Lakelands]. She's seen others doing it and wants to do it but can't' (I:11/9:615). The DT stated:

I've tried her with crafts but she's not good with her hands.... She's not good at quizzes or bingo. The first time I had her playing bingo I realised how bad her dementia was...she hasn't a clue...I haven't tried anything else yet. (I:22/9:873, 877)

Alana's basic ADLs were limited, "Did you know she cannot dress herself? This surprised me as she is such a lovely lady, if you put her clothes on the bed she couldn't put them on" (HCA:I:1/10:999). She also needed assistance with eating, showering, and occasionally toileting. She functioned at Stage 6 on the FAST (Reisberg, 1988, 2007) and the GDS (Auer \& Reisberg, 1997), the penultimate dementia stage where "individuals lose basic activities of daily life capabilities and in which behavioural disturbances peak in occurrence and severity" (Auer \& Reisberg, 1997, p. 168) and has been equated to a clinical diagnosis of moderately severe dementia (Reisberg, 2007).

\section{Engagement in gaming}

Alana had previous experience of using a computer, including computer gaming, although she could not remember or self-initiate, she engaged well with assistance. 
Following assisted initiation, Alana required verbal cues every 5-10 seconds. She was a slow and controlled gamer and by the ninth gaming session she was having increasing intra-game moments of independent gaming agency lasting on average 30 seconds. She did not seem to be unduly affected by the drift associated with the Able-X controller, but needed assistive touch to correct it; otherwise, she would play on in error. Alana played 12 solo gaming sessions: Circuit $(n=12)$, Target $(n=12)$, Mosquito Splat $(n=12)$, Butterfly Catch $(n=12)$ and Popaloon $(n=12)$. Gaming with Alana was interjected with moments of quiet conversation, chatty reminiscence, and periods of quiet concentration, focus, and reflection. 'It's fun, something to work for, it's not just putting this here and this there [I got the impression she was referring to activities that she doesn't see value in like bingo] (VL:30/9, 32:42).

Alana enjoyed and interacted with the positive on-screen messages, which at times triggered reminiscence. The positive on-screen messages were a powerful motivational influence on Alana, she enjoyed praise and flattery and would celebrate her success by self-congratulation: "I feel like a winner" (VN:29/9:1698), and, "I'm becoming a genius [laughed]" (VN:25/9:1649), or by patting herself on the back (VN:22/9:1597, 10/9:1483). At nearly every gaming session, the positive messages or the gaming experience would trigger reminiscences such as memories of school days, "where they always encouraged me to do more" (VN:29/9:1690). When the memories were triggered, gaming was paused to provide a space for reflection and sharing.

Disengagement from gaming was primarily due to on-screen error messages, which would cause Alana to freeze and momentarily disengage, creating moments of gaming inertia and gaming errors as her focus, concentration and confidence were interrupted. She would say, "sometimes I think I am doing it wrong" (VL:1/10:33:35) or, "I'm aware I'm not thinking, am I doing it right?" (VN:1/10:1740). Fortunately, she did not attract too many error messages, so she always left the gaming room alert, bright, and chatty, and this demeanour was maintained beyond the gaming room. Her NOK stated, "It's lovely for her to have an experience [gaming] that she wasn't going to fail, or whatever she did wouldn't be wrong" (NOK:6/10:1407). 


\section{Gaming outputs}

Alana's gaming ability was variable over the gaming period; however, she achieved improvement in most games, including Circuit and Target, which focus on quality of movement and hand-eye coordination (Figure 16).

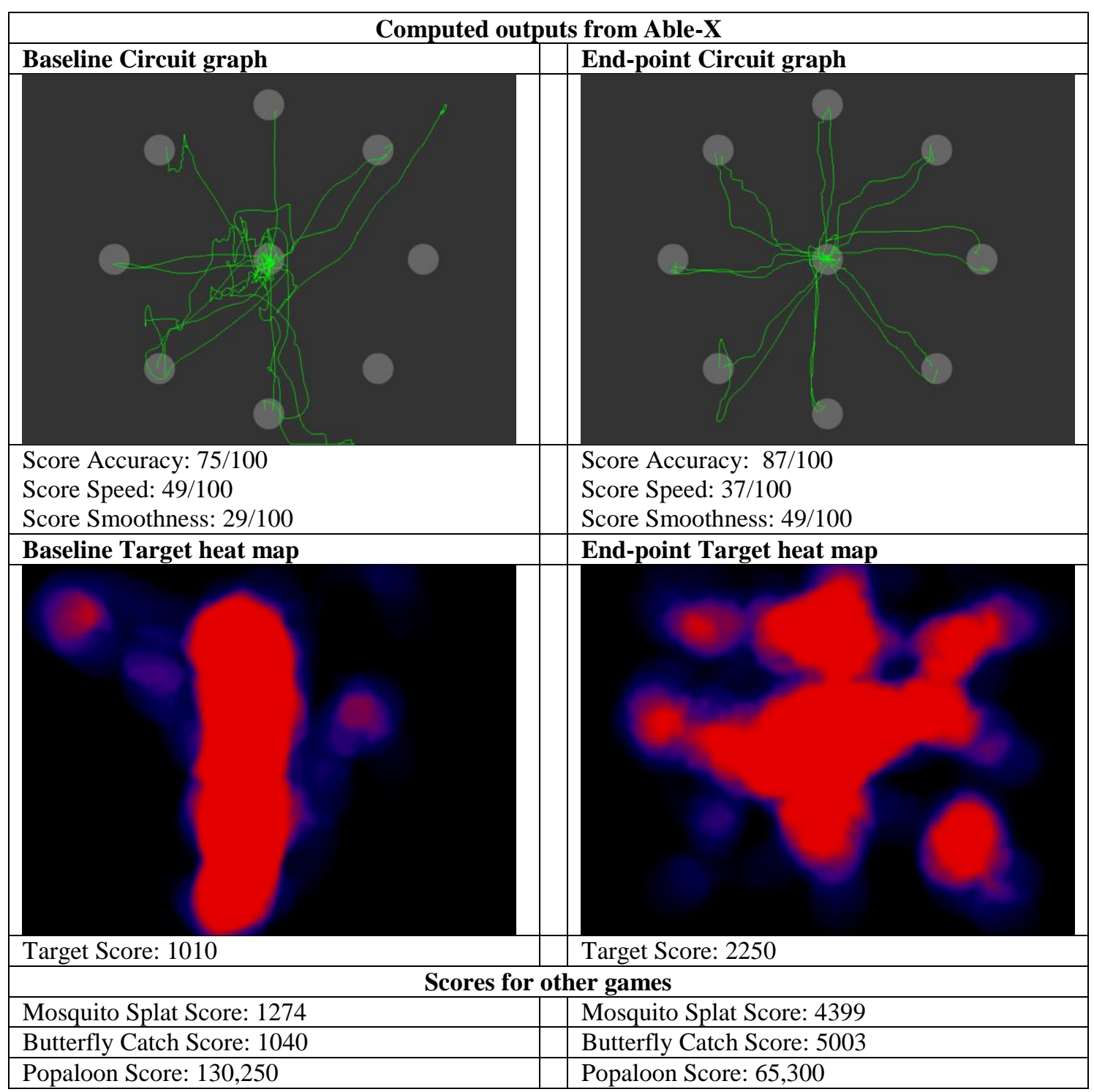

Figure 16. Alana: Computer-generated exergaming outputs

As can be seen on the Circuit graph the baseline assessment is incomplete, because during this gaming session, Alana complained that the assessment gave her headache, so it was abandoned before the final two outer targets were reached. The image, however, speaks for itself in terms of accuracy and smoothness of movement, both of which were improved; however, improvement came at the cost of a slower speed score. 
The Target heat maps show that Alana had extended her play from centre up and down movements at baseline to a wider field of play by end-point, utilising most of the screen, other than the bottom left area. The improved score for this game was not surprising as Alana was gaming without error, with only occasional verbal assistance on this game by session three. Improved Circuit assessment and Target scores infer improved hand-eye coordination in this case. Alana's gaming success continued with improved scores for Mosquito Splat of 3125 and Butterfly Catch of 3963, further inferring improved hand-eye coordination. However, there is no claim of improved visual discrimination in this case as Alana was unable to avoid non-target on-screen images without verbal cues. Conversely, for Popaloon, her score reduced by 64,950 points at end-point. In this game, she needed full verbal support and it took her an average of 2.5 minutes to find patterns herself. It was visually obvious that she did not initially understand what she was doing in this game, although three minutes into the game, she remembered how to play and had moments of independent gaming agency (VL:1/10:26:46), she often said 'I still can't attach my brain to what I am doing [in the game Popaloon]” (VL:1/10:29:28).

\section{Comparing baseline measures and end-point measures}

Alana's cognitive MMSE 2-BV score improved by one, this was in registration and repetition. All other areas were unchanged (Table 27).

Table 27. Alana: Summary of baseline and end-point measures

\begin{tabular}{|l|r|r|}
\hline Validated measure & Baseline & End-point \\
\hline MMSE 2-BV & $3 / 16$ & $4 / 16$ \\
\hline RAID & $4 / 18$ & $3 / 18$ \\
\hline CSDD & $2 / 38$ & $0 / 38$ \\
\hline QOL-AD: Self-report & $42 / 52$ & $47 / 52$ \\
\hline QOL-AD: RN Proxy & $36 / 52$ & $44 / 52$ \\
\hline QOL-AD: NOK Proxy & $37 / 52$ & $40 / 52$ \\
\hline
\end{tabular}

This MMSE score is not unusual for Stage 6 on the GDS (Reisberg, 1988 and Auer \& Reisberg, 1997). However, Alana had insight, "I don't always attach my brain and thoughts to what I am doing and this [gaming] is making me do that...this is what I should be doing” (VN:18/9:1578). She told me she had tried other things without success but she can, "see and feel that gaming is helping... [and she] can feel something is going on [she puts both hands to her temples and taps them]" (VL:18/9:14:44). 
At the beginning of the study, Alana was showing signs of anxiety with a RAID score of 4/18. She scored one point for being frightened/anxious and sensitive to noise, and two points for the aspect of restlessness. By the final assessment Alana's score was $3 / 18$, implying a slightly reduced level of anxiety in dementia, although the aspects of concern had changed. She was no longer frightened or sensitive to noise and her restlessness had reduced, by one point, however, two new concerns were revealed, physical health and memory, for which she was scored one for each item on the RAID. Alana was never able to articulate what it was about her physical health that she was worried about, although her NOK proposed it could be to do with her inability to use her hands, and possibly her insight into her increasing disability. However, there was a little more evidence supporting improved anxiety levels: "She's a little bit calmer... She is doing less of the picking [of her clothes and face] (HCA:I:1/10:1005, 977) and, "Her behaviour is more natural and socially acceptable" (FN:1/10:1094). Moreover, the RN reported:

She was actually quite clingy and touchy, you can almost say flirty... whereas now she is a lot less anxious, so less clingy....[Her] tolerance is much higher, and agitation is much lower, so she's actually very calm, very placid....Definitely less agitated and less wandering. (I:2/10:1141,1143,1247)

Similar scores for anxiety and restlessness were revealed by the CSDD at baseline, but by end-point assessment they were absent. Other evidence demonstrating reduced levels of anxiety, depression, and other BPSD in this case were elicited:

When she first came she was really depressed...[now] she has a more positive mood...she looks forward to the day ahead... her emotions are a little bit better [in this last month]....Emotionally she was needy with hugs, she could not stop...but now she is more appropriate ... less disinhibited. (HCA:I:2/10:1049, 1088)

The QOL-AD assessment revealed additional changes. Overall QOL had improved at the end-point assessment according to Alana's self-rating by five points. The RN concurred and rated eight extra points, similarly, the NOK rated three extra points for this item (Table 28). Additionally, all agreed that the items of 'Friends' and 'Ability to have fun' were improved, and that both items at end-point assessment were excellent. 
Other supporting evidence for improved friendships included: "She's more confident and social [in the last four weeks]....She likes to sit and talk and smile with anyone" (HCA:I:1/10:1083, 1070). Increased socialisation is reiterated by the NOK and the RN who reported, “there's more of a capability now than I ever recall....She's developing friendships, relationships, her little gang, her little clique. She didn't have that for the first 2 months" (I:2/10:1152).

Table 28. Alana: Quality of life points change pre-and post-gaming

\begin{tabular}{|c|c|c|c|c|c|c|}
\hline Items on the QOL-AD questionnaire & $\begin{array}{l}\text { Self } \\
\text { B }^{*}\end{array}$ & $\begin{array}{l}\text { Self } \\
\mathbf{A}^{* *}\end{array}$ & $\begin{array}{l}\mathbf{R N} \\
\mathbf{B}^{*}\end{array}$ & $\begin{array}{l}\mathbf{R N} \\
\mathbf{A}^{* *}\end{array}$ & $\begin{array}{l}\text { NOK } \\
\text { B }^{*}\end{array}$ & $\begin{array}{l}\text { NOK } \\
\text { A }^{* *}\end{array}$ \\
\hline Physical health & 4 & 3 & 3 & 3 & 3 & 3 \\
\hline Energy & 3 & 4 & 3 & 4 & 3 & 3 \\
\hline Mood & 4 & 3 & 3 & 3 & 3 & 3 \\
\hline Living situation & 4 & 4 & 2 & 4 & 3 & 3 \\
\hline Memory & 2 & 3 & 1 & 2 & 1 & 2 \\
\hline Family & 4 & 4 & 4 & 4 & 4 & 4 \\
\hline Marriage (closest relationship) & 4 & 4 & 4 & 4 & 4 & 4 \\
\hline Friends & 3 & 4 & 3 & 4 & 3 & 4 \\
\hline Self as a whole & 3 & 4 & 3 & 4 & 3 & 3 \\
\hline Ability to do chores & 3 & 3 & 3 & 3 & 3 & 3 \\
\hline Ability to do things for fun & 3 & 4 & 3 & 4 & 3 & 4 \\
\hline Money (not relevant to gaming) & 2 & 3 & 1 & 1 & 1 & 1 \\
\hline Life as a whole & 3 & 4 & 3 & 4 & 3 & 3 \\
\hline Total Score & 42 & 47 & 36 & 44 & 37 & 40 \\
\hline Total QOL points change & & +5 & & +8 & & +3 \\
\hline
\end{tabular}

$\mathrm{B}^{*}=$ Baseline score (1=poor, 2=fair, 3=good, 4=excellent). A**=After (end-point score).

Regarding the item 'Ability to do things for fun', the RN reported, "she's [now] often one of the first in that room [activities room] to start the day" (I:2/10:1215). Even the cleaner recounted that Alana asked her if she had seen the "lady with the games" (VN:1/10:1068) as she wanted to play. The fact that she played all 12 sessions is testament that she had the capability to do things for fun. In Alana's own words about gaming with Able-X, "it's fun actually...something to work for...It gives me something to do...and I can do it". I ask her how long the 'I can do it feeling' lasts and she says sometimes for days, or until, it is interrupted by "something nasty... [or something] not fair and it's gone" (VL:1/10:34:54).

Alana rated the item 'Memory' improved by one QOL point from fair to good by the end-point assessment and the RN and NOK agreed. The HCA reported, "she may be a little more alert" (I:1/10:988). The $\mathrm{RN}$ also stated, "she's finding her room a lot better....[and], she seems to be able to focus a bit more" (I:2/10:917 \& 1184). This could 
be attributed to her getting to know her way around and settling down into her relatively new 'Living situation', for which the RN awarded an extra two points at end-point assessment, bringing the RN's score in line with the ratings of Alana and her NOK. Both Alana and the RN agreed that the items 'Self as a whole' and 'Life as a whole', had improved, and excellent at end-point. Moreover, the RN reported that she appears more purposeful in her activities, "she can sit down at the table a lot longer ....Before it was difficult to get her to focus and eat her food [self-feed], she'd often get up halfway into the meal, but now she's engaging more and so we're getting more food into her, so her weight's a bit more stable" (RN:I:2/10:1187). The HCA stated that she seems more confident and able:

Today, she went to talk to [name]. He was sat at the table and all the seats were full, but she stood and talked to him. You have to have confidence to approach his table and stand and talk, that is new for her.... She seems to be able to manage inappropriate attention and relations herself now...there have been no more incidents...she is clearly expressing more confidence. (I:1/10:1078, 1022)

In addition, the HCA revealed that Alana was more purposeful with her hands following gaming:

Today she was outside her room and she was cleaning a mark off the wall. I asked her if she was doing her housework and she said 'yes'.... We noticed that she has started cleaning...in and around her room. She did it when she first came but stopped soon after. She has gone back to what she used to do...house-proud she is. (I:1/10:1014, 1029)

Alana had been a resident for only two months at the commencement of this study and may have been still in the settling down phase, she was also taking citalopram for depression and donepezil for dementia, all of which could have contributed to some of the changes presented in this case.

\section{Case summary and postscript}

Gaming with Able-X resulted in improved QOL outcomes for Alana. The two key findings in this case were improved hand-eye coordination and improved BPSD. Before 
gaming with Able-X, the NOK reported that Alana's ability to use her hands for activities had slipped away. During the gaming period, Alana's ability to use the Able-X controller, including the trigger, which requires finger dexterity, and her concentration improved to the level of moments of independent gaming agency. This improved skill was transferred to the dining room where she reconnected to previous experience of using cutlery at the dining table to self-feed, and her very low weight started to increase as a result. In terms of improved BPSD, Alana was less restless and more settled in place for longer periods of time.

The RN reported that improved hand-eye coordination and improved BPSD changes were maintained for three months. In particular, Alana re-learned capability to use instruments (knife, fork, and spoon) was maintained for the period she stayed at rest home. She continued to be settled at the table and feed herself, "She gained about 3-5kgs in the period between beginning gaming and December 2014" (RN:I:5/7:1453). In January 2015, three months post gaming, an annual review and family meeting was held, and a decision was made to "stop all non-lifesaving medications, including donepezil and citalopram” (RN:I:5/7:1803). Alana became unsettled and was prescribed a PRN antipsychotic (either Quetiapine or Risperidone, the RN could not recall which). Rapid decline followed so the treatment was discontinued. By February, "atypical behaviour had started to reappear and ... she was in a state of rapid decline.... She absconded twice... a decision was made to relocate her to a facility that had a secure dementia bed" (RN:I:5/7:1792, 1802). She was resettled in March 2015 and the NOK reported, "It was an awful time for Mum and me during the last few months at [Lakelands]. I think Mum was her happiest during the time you saw her. I would say a lot was to do with enjoying interacting with you and the special care you took to spend time with her" (NOK:email:21/7). Last words from Alana: "I've enjoyed it [gaming]...I've enjoyed spending time with you, it gives me a twinkle in the heart" (VN:1/10:1781). RIP December 2016.

\subsubsection{Florence $^{34}$}

She's a fantastic girl...full of life, she's energetic....The girl loves to laugh....She's very rarely not happy.... She loves life... but she's a little forgetful. (RN:I:9/9:7)

\footnotetext{
${ }^{34}$ Pseudonym: Florence means "Prosperous, flowering" (http://www.thenamemeaning.com/florence/)
} 


\section{Personal and clinical profile}

Florence was a larger than average build, average height, octogenarian (83) female, born in NZ. In 2011, at the age of 80, Florence began to have minor strokes. Over the next two years she started "shutting herself out...she was embarrassed because she'd forget things, and some people [at the 60s Club] would snap at her" (NOK: I: 15/9:947). She had stopped cooking, would not eat the meals that had been organised, and was angry with her daughter if she left reminders on the fridge door. On two occasions, Florence became lost in a shopping centre. On a third occasion her neighbour found her asleep on a couch in a shopping centre. "The problem was the...neighbour ... was actually covering up for Mum a bit, so wasn't actually helping her" (NOK:I:15/9:1029). In late 2013 Florence repeatedly presented at the GP surgery for "appointments she hadn't made, or [was] not turning up for appointments she had made" (NOK:I:15/9:1011). In December 2013, she had another stroke, which affected her short-term memory, a diagnosis of VaD was made by the GP, and plans were made to relocate her to Lakelands rest home level of care where she could receive appropriate support. Florence settled in place quickly: "I used to think I'm useless...I worried about becoming a vegetable.... I might as well be dead, but not now, I am happy with life and where I live” (VN:11/9:799).

In July 2014, when this study commenced, Florence had been resident for eight months. She self-mobilised with the aid of a walking stick. Her mobility was slow and her gait had an exaggerated lurch to either side as she walked. This was possibly a result of her several strokes and the effects of three total knee replacements. Balance problems are not unusual following a stroke and with people with $\mathrm{VaD}$ (Stephan \& Brayne, 2014; Ekberg et al., 2002; Perkins, 2013). However, she was independent and very proud to be so, stating, "I go for a walk along here, and right along there [pointing down the corridors and around the building]" (F:I:9/9:1364).

Florence was independent with all ADLs, however, IADL were more of a challenge. She could no longer use the telephone, although she could still turn on her TV and music centre, so had retained some instrumental capability. "I'm a fussy old thing. Even here, I like my room tidy and do my own dusting and all that" (F:I:9/9:1345). Her IADLs capabilities extended to shopping for personal items, usually in the company of her daughter, who was her NOK and EPA for Personal Care and Welfare. She functioned at Stage 4 of the FAST (Reisberg, 1988, 2007) and the GDS (Auer \& Reisberg, 1997). Stage 
4 is where "individuals generally have deficit in management of instrumental activities of daily life" (Auer \& Reisberg, 1997, p. 168) and has been equated to a clinical diagnosis of mild dementia (Reisberg, 2007).

\section{Engagement in gaming}

Florence had never used a computer before so gaming on the computer was a new experience for her. She engaged eagerly in solo gaming sessions and played: Circuit $(n=12)$, Target $(n=12)$, Mosquito Splat $(n=12)$, Butterfly Catch $(n=12)$ and Popaloon $(n=2)$. During the first gaming session, it was obvious that Florence was going to find it challenging to use the Able-X controller as her upper torso moved in the direction of her eyes, and her hands, wrists and arms (usually bent at the elbows) were pinned tightly into her lateral torso and folded across her middle trunk (VL:10/9:04:12), so mastery of the Able-X controller was a major feat. The natural drift associated with the controller exacerbated her exaggerated upper-body movements, and she needed constant assistive touch by me to reduce the drift as well as varying levels of verbal support to facilitate her gaming agency. During the first session Florence became upset as her body sway became exaggerated during gaming (VL:10/9:3:33, 04:12, 06:55), she had no centre of gravity and appeared unstable on the chair. The DT was present but left after a few minutes with a shake of her head in my direction, she reported later, "She is worse than I thought" (I:1/10:598). Florence needed near constant verbal reminders to move her arms freely away from her torso and bend and roll her wrists, otherwise they were held stiffly in place and gaming was restricted (VL:10/9:1:00). Gaming with Florence was constantly interjected with my reminders, demonstrations and repeated practice sessions on how to move her wrists and arms freely (VL:11/9:07:00-08:00, 15/9:00:00-01:00, 16/9:00:00). Similar age and health related motor impairments were found by Boulay et al. (2011).

By the second gaming session, Florence recognised that she was using her whole upper body rather than her arms and wrists and started to self-correct (VL:11/9:03:55). Her NOK reported, "I can actually remember her doing something like that [swaying movement] with one of the kids at school [when watching her grandchild use a computer]" (I/6/10:675). No one else reported this exaggerated hand-eye-body dissonance, although the RN reported at baseline, "her mobility is just starting to decline" (I:9/9:77). 
As gaming progressed, Florence began to enjoy simple time limited games and repeated success of easy gaming. She was motivated by praise (VL:10/9: 08:30; VL:16/9:27:00), positive on-screen messages and the contemporaneous computergenerated scores, which made her laugh out loud (VL:16/9:23:14, 29/9:17:00) and visibly flush with pride. Florence commented, "I can't believe I've done so well" (VL:16/9:26:40), and would express how gaming made her feel, "It [gaming] makes me feel good...I no longer feel stupid [and]...it makes me feel really good and not useless" (FN:1/10:620, 626). I asked how long this feeling lasts and she said, "it stays with me...It makes me think I can do stuff" (VN:25/9:2070, 2059). A change was also noticed by the HCA, "I don't know what you are actually doing in these games but she has been very proud [and] very happy with herself (I:1/10:251).

The level of assistance needed by Florence to achieve gaming agency reduced markedly over the gaming period. By the fourth session, she had progressed from near full assistance with initiation and gaming, to gaming with verbal assistance only, and had moments of independent gaming agency (VL:15/9:06:04). She used her lower arms and wrists well, her central body core was stable, and her body posture much improved (VL:15/9:13:57). She started to self-evaluate as she recognised improvement and stated, "I am getting better [at gaming]" (VN:15/9:1876). By the $12^{\text {th }}$ and final gaming session she had extended moments of independent gaming agency and her improved body posture was obvious both inside and outside the gaming room. She needed constant positive regard, which motivated her (VL:10/9:16:02). She would regularly say, "I can't believe it [smiling and laughing]” as I praised her efforts (VN:10/9:1722).

During the gaming sessions, there were two reasons for gaming disengagement: poor hand-eye-body coordination and negative on-screen messages. The former made it particularly difficult for her to manage the Able X controller, especially the trigger, which she never learnt to use. She was also distracted by negative on-screen messages, and her distraction was evident by a look of disheartenment on her face. She would also express her frustration (VL:18/9:10:00) and/or have moments of gaming inertia, which was usually followed by a run of gaming errors until confidence and gaming agency was regained (VL:8/9:10:00, 1/10:09:23) through the provision of continual positive regard. 


\section{Gaming outputs}

Florence's gaming ability and scores were variable; however, her most improved scores were for the assessment Circuit and the game Target, both of which are clearly demonstrated on the Circuit graph (Figure 17):

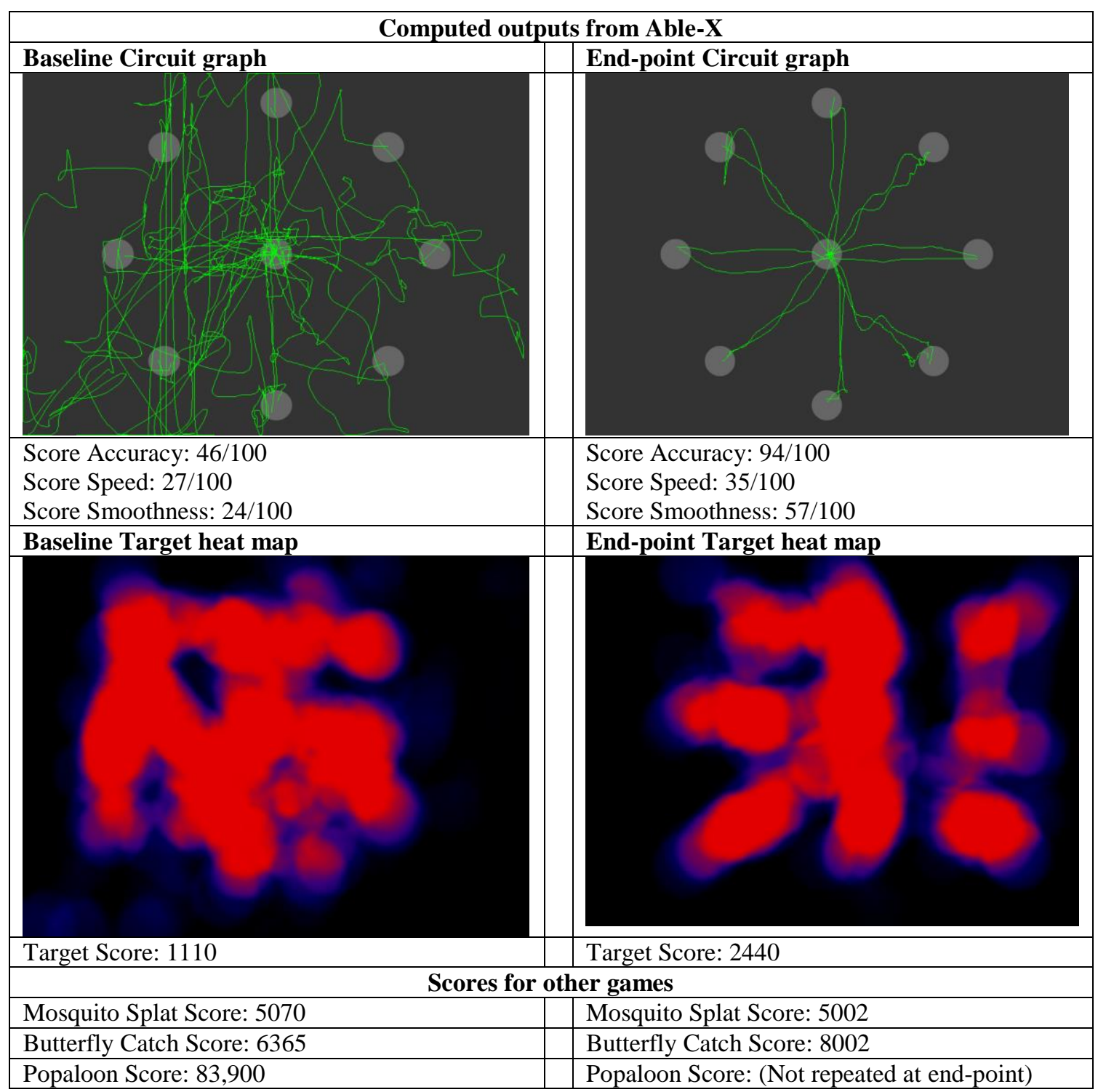

Figure 17. Florence: Computer-generated exergaming outputs

She also had a slight improvement in speed to target without reduction in accuracy or smoothness. For the game Target, she more than doubled her score, and as the Target heat map demonstrates, she was more active, especially on the right side of the gaming screen. Both Circuit and Target scores indicate improved hand-eye coordination over the gaming period. Improvements in hand-eye-body coordination was also evident on video footage (VL:10/9:3:3, 15/9:13:57) and on reports from others. 
Gaming on Mosquito Splat, Butterfly Catch and Popaloon were more variable. Florence had a slightly reduced score for Mosquito Splat, but did a little better on Butterfly Catch with an improved score by 1637 points, further indicating improved hand-eye coordination. However, Florence needed verbal assistance to avoid non-target on-screen images, so there is no claim of improved visual discrimination in this case. I also noted that when playing Mosquito Splat and Butterfly Catch, Florence could use the Able-X controller in a vertical (up and down) gaming position which worked well for her, not because she was aiming to catch the on-screen targets, but because on-screen targets flew into her vertical path as they crossed the gaming screen.

The final game, Popaloon, was near impossible for Florence to play and this was evident by the second gaming session. Florence found it virtually beyond her capability to use index finger manipulation to pull and release the trigger on the Able-X controller (VL:11/9:24:00, 26:36). This lack of ability affected her gaming confidence and the cacophony of gaming inertia moments which made her feel that she was doing something wrong (VL:11/9:28:35), and it was clear she was becoming stressed. In the ethical interest of doing no harm during the intervention (Drury et al., 2007; Spector et al., 2000; Woods et al., 2012), this game was abandoned and removed from Florence's gaming routine. Also, the constant repetitive instruction was exhausting for me as researcher. The threat of exhaustion and the need to have coping strategies is well documented in qualitative research (Dickson-Swift et al., 2006, 2007; Wray et al., 2007) and forgoing Popaloon in this case benefited me also.

\section{Comparing baseline measures and end-point measures}

Florence's cognitive MMSE 2-BV score deteriorated at end-point by two points, consisting of one point loss for registration and for orientation to time (Table 29). Both MMSE scores are unusually low for Stage 4 global decline (Auer \& Reisberg, 1997; Reisberg, 1988). Her NOK was not surprised by the low MMSE score and stated, "Mum is changing from the mum that we've known over the years, which is hard.... We have noticed her memory is worse now, even since she 's been in [Lakelands]" (I:15/9:939, 957). 
Table 29. Florence: Summary of baseline and end-point measures

\begin{tabular}{|l|r|r|}
\hline Validated measure & Baseline & End-point \\
\hline MMSE 2-BV & $6 / 16$ & $4 / 16$ \\
\hline RAID & $5 / 18$ & $1 / 18$ \\
\hline CSDD & $1 / 38$ & $0 / 38$ \\
\hline QOL-AD: Self-report & $46 / 52$ & $42 / 52$ \\
\hline QOL-AD: RN Proxy & $37 / 52$ & $45 / 52$ \\
\hline QOL-AD: NOK Proxy & $36 / 52$ & $47 / 52$ \\
\hline
\end{tabular}

Conversely, the DT reported slight improvement in cognition:

Her short-term memory is really bad, but this morning I brought my England onesie... as it is England Day and she said, 'Oh, I remember you wore that when those two came from England [Prince William and Princess Kate] and I remember you came back really wet'. She was spot on. She had that memory and I thought wow. (I:1/10:529)

Improved remembering was also evidenced during gaming, by the last session she was recalling playing the games before and how to play them (VL:1/10:15:12). The DT noticed, "She hasn't repeated herself as much. Yes, definitely less repeating and more remembering things" (I:1/10:613) and the RN reported that Florence remembered gaming and would look for me when I was in the facility. Conversely, her NOK stated, "Mum hasn't mentioned the gaming at all [unless prompted]." The HCA reported her level of confusion was reduced, the RN concurred, "no...confusion in the last month, just more of a determination" (I:2/10:403). However, Florence's NOK thought that there was no change in her confusion levels. Improved remembering and less repeating is evidence of improved BPSD (Bpac $\left.{ }^{\mathrm{nz}}, 2013\right)$, which is an indicator of improved QOL.

According to the RN, at the beginning of the study Florence was showing signs of mild or intermittent anxiety in the items of physical health, cognitive performance, worried over trifles, sensitivity to noise and fatigability, having an overall RAID score of 5/18. On reassessment, the end-point score was 1/18. All perceived worries had subsided apart from concern about physical health (discussed below). The CSDD implied mild or intermittent anxiety at baseline assessment with a score of $1 / 38$, but by end-point reassessment the score was $0 / 38$. Other evidence of improvement in anxiety noted by the DT were, "she's happier, she's laughing more, and asking for activities to do" (I:1/10:575); the RN and 
HCA concurred. The HCA reported on improved self-esteem and recounts that Florence informed her "she can do more than she thought she could do" (I:1/10:267). Improvement in levels of anxiety level and depression demonstrate improvement in BPSD (Bpac ${ }^{\mathrm{nz}}$, 2013).

Overall Florence's QOL-AD assessment had improved according to the ratings of the RN by 8 QOL points and the NOK by 11 points. Conversely, Florence rated her QOL to be poorer by four points (Table 30).

Table 30. Florence: Quality of life points change pre-and post-gaming

\begin{tabular}{|c|c|c|c|c|c|c|}
\hline Items on the QOL-AD questionnaire & $\begin{array}{l}\text { Self } \\
\text { B }^{*}\end{array}$ & $\begin{array}{l}\text { Self } \\
\mathbf{A}^{* *}\end{array}$ & $\begin{array}{l}\mathbf{R N} \\
\mathbf{B}^{*}\end{array}$ & $\begin{array}{l}\mathbf{R N} \\
\mathbf{A}^{* * *}\end{array}$ & $\begin{array}{l}\text { NOK } \\
\text { B* }\end{array}$ & $\begin{array}{l}\text { NOK } \\
\text { A }^{* *}\end{array}$ \\
\hline Physical health & 3 & 3 & 2 & 3 & 3 & 4 \\
\hline Energy & 4 & 3 & 2 & 4 & 2 & 4 \\
\hline Mood & 4 & 3 & 4 & 4 & 2 & 3 \\
\hline Living situation & 3 & 4 & 3 & 4 & 4 & 4 \\
\hline Memory & 2 & 2 & 2 & 2 & 2 & 2 \\
\hline Family & 4 & 4 & 3 & 3 & 3 & 4 \\
\hline Marriage (closest relationship) & 4 & 4 & 3 & 3 & 4 & 4 \\
\hline Friends & 3 & 2 & 4 & 4 & 3 & 4 \\
\hline Self as a whole & 4 & 4 & 3 & 4 & 3 & 4 \\
\hline Ability to do chores & 4 & 3 & 2 & 3 & 3 & 4 \\
\hline Ability to do things for fun & 4 & 3 & 3 & 4 & 3 & 4 \\
\hline Money (not relevant to gaming) & 3 & 3 & 2 & 3 & 1 & 2 \\
\hline Life as a whole & 4 & 4 & 4 & 4 & 3 & 4 \\
\hline Total Score & 46 & 42 & 37 & 45 & 36 & 47 \\
\hline Total QOL points change & & -4 & & +8 & & +11 \\
\hline
\end{tabular}

All agree that 'Self as a whole' and 'Life as a whole', were excellent at end-point. Moreover, Florence appeared to have new found confidence, assertiveness and determination that was recognised: "she appears to have a high self-esteem and reports she can do more than she thought she could do...It's great" (HCA:I:1/10:267), and "maybe a little more confident....She is taking the initiative" (DT:I:1/10:629). For the items 'Energy' and 'Mood', Florence reassessed these items slightly lower at the end-point assessment, grading herself as good. Conversely, both the RN and NOK thought the item 'Energy' had improved by two points at end-point reassessment and was excellent.

Likewise, with the item 'Mood', the RN rated no change for this item, which she rated as excellent, and her NOK rated this item to be improved from poor to good. Florence rated her 'Ability to do chores' and 'Ability to do things for fun' to be reduced 
by one point for each item. The RN and NOK differed in their assessment and rated these items to be improved by one point. The $\mathrm{RN}$ reported, "in the last month ... a bit more of a have-a-go attitude, which you once would have never associated with her" (RN:I:2/10:404). This newfound assertive and have-a-go attitude was deemed by the HCA to be "overpowering" (I:2/10:195). Florence also rated the item 'Friends' to be slightly reduced at end-point assessment, although the $\mathrm{RN}$ rated this item to be excellent and stated, "she's very well loved, everybody likes to engage with her....She's our Queen" (RN:I:23/9:22, 135). The NOK also rated this item as improved from good to excellent at reassessment.

The final and most important aspect in this case is the change in 'Physical health'. Florence perceived this item unchanged at end-point reassessment, rating herself good. The RN awarded an extra QOL point on reassessment, aligning her score with that of Florence. The NOK also awarded an extra point at reassessment rating this item excellent at the end-point assessment. The biggest change was in physical ability brought about by improved hand-eye-body coordination, which extended beyond the gaming room. This improvement was reported on by the $\mathrm{RN}$, "If you look at her gait, it's a lot more upright...she seems to be taking longer steps, stronger stride.... We have noticed [since gaming] that she seems to be a bit more assured in her mobility" (I:2/10:323). So improved was Florence that the RN agreed to her request to take her out on a longer than usual walk (100-200 metres):

She didn't just walk up and down the drive, she was the only one who was mobile, the rest were all in wheelchairs so she was the slowest...but we waited.... She got right to the bottom...the best part of 400 metres...I said, my car's here, do you want me to give you a lift [back]? 'Bugger off', she said, 'I'm gonna do that hill'....She was knackered, when she got into the hallway, but she was beside herself. (RN:I:2/10:463)

The following day Florence was waiting by the door for me and she proudly recounted the above story. I asked her what made her think she could do the long walk and she said, "I realise, from this game, that I can improve, and I'm not stupid, so I set myself a goal" (FN:2/10:458). The HCA reported, "I know she is feeling very proud of 
herself because she's been out for some walks.... She's feeling very happy with herself and she told me this twice" (I:1/10:251). In addition, her NOK reported:

We usually go walking to the plaza from [one end to the other]. She'd have to stop two times, but I asked her if she's alright without stopping and she said that she was all fine and that she's walking a lot better [since gaming]. (NOK:I:6/10:682)

\section{Case summary and postscript}

Gaming with Able-X resulted in improved QOL outcomes for Florence. Her key improvements were hand-eye-body coordination, increased confidence and more determined personal agency. Before gaming with Able-X, the RN reported Florence's mobility was in decline. During the gaming period, marked improvements in hand-eyebody coordination and mobility were recorded and reported, both in computer-generated outputs and by others who knew Florence well.

Florence's ability to use the Able-X controller consistently improved to the level of extended moments of independent gaming agency, which boosted her confidence. She used her newfound confidence assertively to create an opportunity to achieve her goal to extend her usual walking space and walking distance, successfully completing a walk of 800 metres, which included a 400-metre relatively steep climb, and a similar distance in a local shopping mall. Staff and NOK reported improvements in posture, gait, stride, and mobility. Also improved was her self-esteem, which resulted in increased assertiveness according to the RN. Florence began to project her views, demonstrating independent agency that extended beyond the gaming room and into everyday life. She interacted with a wider environment, through mobility and her personal agency to be more assertive, engaged, and motivated. Improved motivation, self-esteem, confidence, and depression and anxiety scores are all associated with improved BPSD (Bpac $\left.{ }^{\mathrm{nz}}, 2013\right)$.

Ten months post gaming; all improvements identified above were maintained. She continued to maintain improved hand-eye-body coordination, beyond the level of pregaming functioning. The RN reported, "at a family meeting, improved mobility and reduced depression [was recognised by all present, including the GP] and her dependency score reduced, which is a very rare occurrence" (I:5/7:1628). In terms of newfound confidence, voice, and assertiveness the $\mathrm{RN}$ reported, "She continues to be 
assertive...outspoken... [and] very independent.... She has come out of the corner and is comfortable in the middle of the room" (I:5/7:1633). Last word from Florence: "This [gaming] is lovely. It keeps my mind active; I could play all day....It's wonderful, it makes me feel good. It makes me feel happy" (VN:12/9:1858; VN:29/9:2102).

\subsection{Chapter summary}

This chapter has explored the computer-assisted exergaming experiences of 10 people with dementia. Each unique case documents the ways in which participants engaged with the Able-X computer-assisted exergame system. It reveals how all participants, irrespective of cognitive ability or severity of dementia, could participate in computer-assisted exergaming therapy with varying levels of support that empowered them to become gaming active and to complete the gaming challenges. Furthermore, analysis of baseline and end-point assessments, observations, and reports revealed improved hand-eye coordination and improvement in some items of QOL. Each CS in this collective is unique; however, cross-case analysis identified similarities and differences across the cases. These findings are presented in the following chapter. 


\section{CHAPTER FIVE: CROSS-CASE ANALYSIS}

\subsection{Introduction}

This chapter presents the similarities and differences across the 10 cases studies described in Chapter Four. Collectively, both chapters answer the research question: How does Able-X affect QOL, cognitive functioning and physical functioning in people $\geq 65$ years, who are living with dementia and residing in three different assisted care settings in NZ? I begin with an overview of the demographic, personal, and clinical characteristics of the cases, and a synopsis of computer-assisted exergaming with Able-X in terms of gaming engagement, frequency, and game description. Following this overview, I utilise a theoretical and analytic framework of 'embodied selfhood' (Kontos, 2003, 2004) and the principle and strategies of EL (Terrace, 1963) to examine in detail five emergent themes and six associated novel concepts (game shy, game enabled, game able, gaming by association, repeated success of easy gaming, and error starer-error inertia). The first three themes have a focus on the exergaming environment: sentient interaction with the environment, exergaming initiation and advancement, exergaming engagement and progress. Themes four and five explore the similarities and differences across each case in terms of the two main findings of improved hand-eye coordination and improved QOL. Throughout the chapter, I provide evidence of how these findings translated from the gaming room into the everyday lives of PWD.

For clarity, I use five terms to reveal embodied selfhood. Three terms are used to evince embodied selfhood emanating from the primordial significance of the human body that has the natural ability to apprehend and convey meaning. They are 'intentional bodily actions/movement/agent/agential', 'intentional bodily gestures', and 'sentient interaction in the environment'. Two terms are used to evince embodied selfhood that emanates from the socio-cultural dimension of the pre-reflective body that implicitly understands the socio-cultural ways of being-in-the-world, due to the priming effect in each person by his/her earliest upbringing. They are 'expressions of socio-cultural etiquette' and 'intentional socio-cultural relationality'. Although each example may show multiple cases of embodied selfhood, I only signpost the one(s) relevant to the sub-heading in which they are presented. In addition, to avoid over referencing the above-named authors, and to 
allow the text to flow, I have decided to signpost the theoretical influences in the first few examples only as I believe the reader will understand which theoretical influence I am drawing upon when interpreting my findings in this chapter.

\subsection{Demographic and personal characteristics}

The demographic, personal, and clinical characteristics of the participants in this study are summarised in Tables 31 and 32. The majority of cases (seven) were female and three were male. Most of the participants (six) were born in NZ, two in the UK, one each in Europe and the Middle East. The representation of $70 \%$ women is slightly higher than expected considering the estimation that women carry $60 \%$ of the dementia disease burden (Alzheimers New Zealand Incorporated, 2008; Santibáñez et al., 2007).

The median age of participants was 83.5 (range 62-91). However, one participant, a stroke survivor aged 62, was younger than the original target population of 65 years and over, but due to difficulty recruiting participants the inclusion criteria were amended. If the youngest participant is removed from the data corpus, the median age is 84 (range 7791). It was not possible to compare the age range with the participants in Kontos' study (2004) as she averaged the age of all 80 residents on the floor where she was conducting her research, instead of the 13 participants whom she recruited. When compared to other similar case studies/case series concerned with cognitive rehabilitation in PWD, the mean age was similar (within in 2-3 years) but slightly older than van Tilborg et al. (2011) and Metzler-Baddeley and Snowden (2005). However, some studies focus on populations at the earlier end of the dementia trajectory who are more than a decade younger (Clare et al., 2000; Thivierge et al., 2008).

All 10 participants in this study had married, six were widowed, and one divorced. Only three had living spouses. Spouses were next of kin (NOK) and enduring power of attorney (EPA) for two participants. One spouse was also a resident and shared a room in the dementia unit with her husband (Thomas). In this case, the NOK/EPA was their son. The divorced participant's NOK/EPA was his brother. For participants now widowed, their NOK/EPAs were their children, daughters for six participants and son for one participant. Even though all but one of the participants had at least one son, their daughters stepped into the caring role, which is in line with the findings of others (de Vries et al., 
2016; Kirkman, 2011; Kerse, 2014a; Ward-Griffin et al., 2007), and with what is known about the burden of caring for a family member with dementia (de Vries et al., 2016; Kirkman, 2011; Kerse, 2014a; Ward-Griffin, Oudshoorn, Clark, \& Bol, 2007).

Inclusion criteria for this study required a degree of functionality in terms of being able to communicate, follow instructions, hold the controller, transfer and mobilise to the gaming room and engage with the computer-assisted exergaming technology, all of which required a degree of physical capability in terms of some basic ADLs, and the more complex IADLs. Three participants were independent in terms of basic ADLs as described by Stineman et al. (2014); the remaining seven had some ADLs deficit, none of which affected their ability to take part in the study (Table 31). Basic ADLs are generally retained until the penultimate stage (Stage 6) of the dementia journey (Auer \& Reisberg, 1997). Four participants were at this stage of global decline (Table 32) but they were still able to engage with, and enjoy, computer-assisted exergaming with Able-X.

IADLs are more complex as they require planning and sequencing capabilities ${ }^{35}$ (Stineman et al., 2014), which are generally being eroded by Stage 4 of the disease trajectory according to Auer and Reisberg (1997). Nine participants had reached this stage of functional and global decline or had progressed beyond it. One participant did not meet the criteria for assessment. All participants had severe IADLs decrements, which had the potential to impact upon the instrumental activity of computer-assisted exergaming with Able-X. I realised during recruitment, that my role was at least tetrachotomous (Huber, 2003): researcher, teacher, game partner, and study subject. My role being synergetic and determined by the level of assistance required by each participant. Within this synergic dyad, all participants could engage with computer-assisted exergaming with Able-X with varying degrees of assistance from me, implying that if the individual can hold the handheld controller or other relevant interface objects, IADLs decrement should not exclude PWD from exergaming with Able-X in partnership with a trained other.

\footnotetext{
${ }^{35}$ For example, the activity of using the telephone incorporates many sequential steps, including locating the phone, selecting a number, using the phone and the number together to make a call (Stineman et al., 2014).
} 
Table 31. Demographic and personal characteristics of cases

\begin{tabular}{|c|c|c|c|c|c|c|c|c|c|c|}
\hline Name & Gender & Age & Ethnicity & $\begin{array}{l}\text { Marital } \\
\text { status }\end{array}$ & $\begin{array}{l}\text { Next of } \\
\text { kin }\end{array}$ & $\begin{array}{l}\text { No. } \\
\text { children }\end{array}$ & $\begin{array}{l}\text { Basic activities } \\
\text { of daily living } \\
\text { (ADLs) }\end{array}$ & $\begin{array}{l}\text { Instrumental } \\
\text { activities of } \\
\text { daily living } \\
\text { (IADLs) }\end{array}$ & $\begin{array}{l}\text { Education } \\
\text { level }\end{array}$ & Occupation \\
\hline Joy & Female & 86 & $\mathrm{NZ}$ & Widow & Daughter & $\begin{array}{l}2 \text { sons } \\
2 \text { daughters }\end{array}$ & Minimal assist. & Partial assist. & $\begin{array}{l}\text { Pre-high } \\
\text { school cert. }\end{array}$ & $\begin{array}{l}\text { Shop worker } \\
\text { and auditor }\end{array}$ \\
\hline Iris & Female & 84 & Netherlands & Widow & Daughter & $\begin{array}{l}1 \text { son } \\
2 \text { daughters }\end{array}$ & Partial assist. & Full assist. & $\begin{array}{l}\text { Pre-high } \\
\text { school cert. }\end{array}$ & $\begin{array}{l}\text { Office worker/ } \\
\text { typist }\end{array}$ \\
\hline Bill & Male & 78 & $\mathrm{NZ}$ & Married & Wife & $\begin{array}{l}2 \text { sons } \\
1 \text { daughter }\end{array}$ & Partial assist. & Full assist. & $\begin{array}{l}\text { Pre-high } \\
\text { school cert. }\end{array}$ & $\begin{array}{l}\text { Security } \\
\text { Warden }\end{array}$ \\
\hline Thomas & Male & 87 & UK & Married & Son & $\begin{array}{l}2 \text { sons } \\
1 \text { daughter }\end{array}$ & Partial assist. & Partial assist. & Tertiary & $\begin{array}{l}\text { Navy/aircraft } \\
\text { mechanic/ }\end{array}$ \\
\hline Maya & Female & 77 & NZ & Married & Husband & $\begin{array}{l}2 \text { sons } \\
3 \text { daughters }\end{array}$ & Partial assist. & Partial assist. & $\begin{array}{l}\text { High school } \\
\text { Certificate }\end{array}$ & $\begin{array}{l}\text { Factory } \\
\text { work/home } \\
\text { maker }\end{array}$ \\
\hline Risa & Female & 83 & NZ & Widow & Daughter & $\begin{array}{l}1 \text { daughter } \\
2 \text { sons }\end{array}$ & Independent & Partial assist. & $\begin{array}{l}\text { Pre-high } \\
\text { school cert }\end{array}$ & $\begin{array}{l}\text { Minister/piano } \\
\text { teacher }\end{array}$ \\
\hline Clara & Female & 91 & UK & Widow & Daughter & 2 daughters & Independent & Partial assist. & $\begin{array}{l}\text { Pre-high } \\
\text { school cert. }\end{array}$ & $\begin{array}{l}\text { Administrator } \\
\text { /Navy }\end{array}$ \\
\hline Alfie & Male & 62 & $\mathrm{NZ}$ & Divorced & Brother & $\begin{array}{l}1 \text { son } \\
3 \text { daughters }\end{array}$ & $\begin{array}{l}\text { Full } \\
\text { Assist. }\end{array}$ & Partial assist. & $\begin{array}{l}\text { High school } \\
\text { Certificate }\end{array}$ & $\begin{array}{l}\text { Tax related } \\
\text { positions }\end{array}$ \\
\hline Alana & Female & 86 & $\begin{array}{l}\text { Middle } \\
\text { Eastern }\end{array}$ & Widow & Daughter & $\begin{array}{l}2 \text { sons } \\
1 \text { daughter }\end{array}$ & Partial assist. & Partial assist. & $\begin{array}{l}\text { Pre-high } \\
\text { school cert. }\end{array}$ & $\begin{array}{l}\text { Doctors } \\
\text { receptionist/ } \\
\text { Administration }\end{array}$ \\
\hline Florence & Female & 83 & $\mathrm{NZ}$ & Widow & Daughter & $\begin{array}{l}2 \text { sons } \\
3 \text { daughters }\end{array}$ & Independent & Partial assist. & $\begin{array}{l}\text { Pre-high } \\
\text { school cert }\end{array}$ & $\begin{array}{l}\text { Sewing } \\
\text { machinist/Home } \\
\text { maker }\end{array}$ \\
\hline
\end{tabular}


Table 32. Clinical characteristics of cases

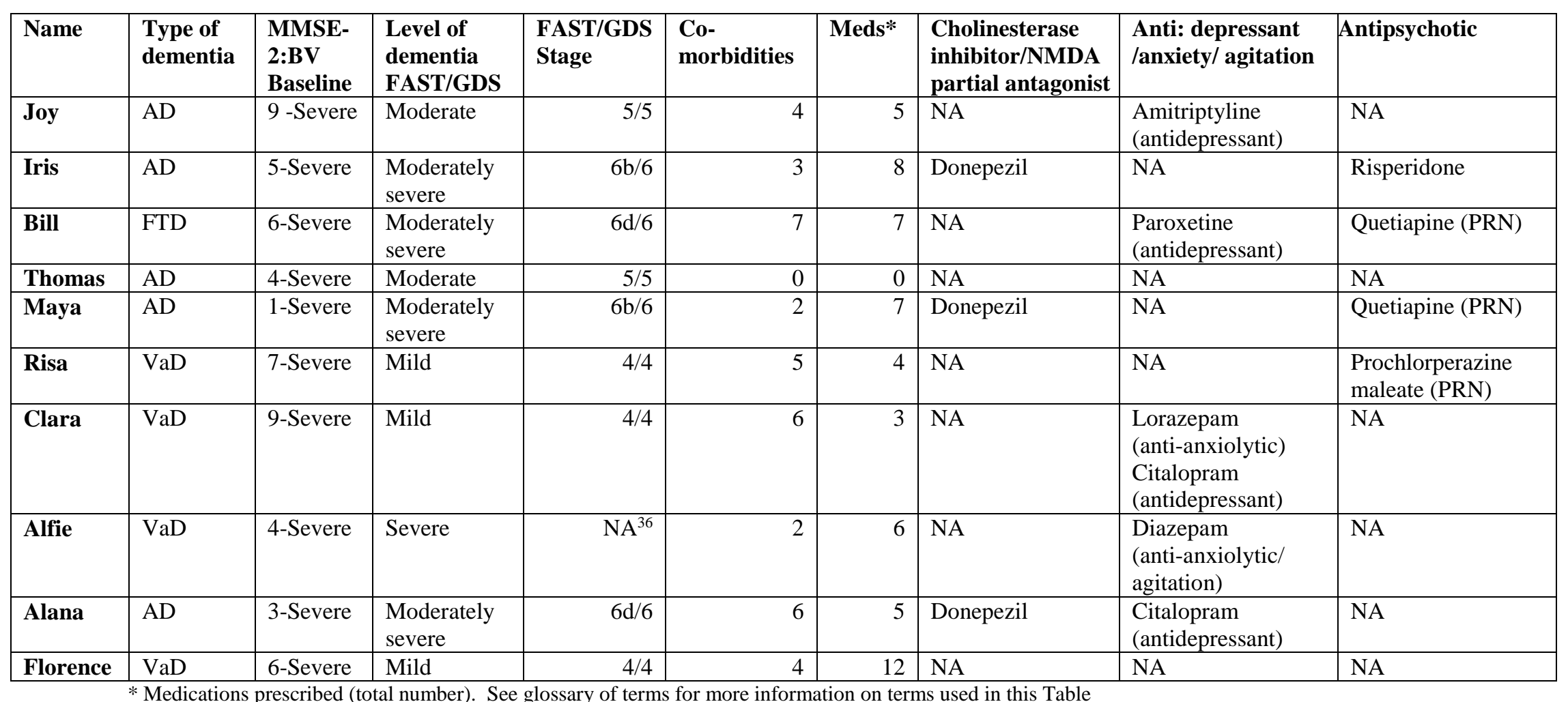

${ }^{36}$ It was difficult to determine which aspects of disability, loss of functioning and decline were related to his multiple strokes, and which were related to vascular dementia, for that reason, they were not appropriate measures for this case. 


\subsection{Clinical characteristics}

The most common type of dementia was $\operatorname{AD}(n=5)$, followed by $\operatorname{VaD}(n=4)$ and one participant had frontotemporal dementia (Table 32). This collective CS has slightly higher representation of $\mathrm{VaD}$ and no people with dementia with Lewy bodies. The AD burden in women 73 years and over was present, with four of the seven women having AD. Three other women aged 82-91 had VaD. The aetiology for men was more diverse, one had $\mathrm{VaD}$, one had $\mathrm{AD}$, and one had frontotemporal dementia.

Participants in this study appeared to have more severe cognitive decrement at baseline compared to similar studies. Laffan et al. (2010) participants had a MMSE score range of 15-25, van Tilborg et al. (2011) 15-26, Clare et al. (2000) 21-26, MetzlerBaddeley and Snowden (2005) 11-26, and Thivierge et al. (2008) 19-25, all of which were classified at mild to moderate level. In addition, the baseline assessments for cognition were surprising to the lead RNs, whom were responsible for selecting participants with mild to moderate dementia. In all cases, their clinical judgment differed significantly from participants' baseline MMSE-2:BV assessment. Banerjee et al. (2006) warns of the risks associated with discrete functional assessment such as cognition, and recommends more global assessments. By re-assessing my participants utilising the FAST/GDS (Auer \& Reisberg, 1997) all but one were re-staged from severe dementia, as determined by the MMSE-2:BV score, to mild for three participants, moderate for two participants, and moderate to severe for four participants (Table 32). By using a more global approach, rather than discrete cognitive assessment, as recommended by Banerjee et al. (2006), and Oyedobe, and Parveen (2016), nine of my participants met the inclusion criteria. One, Alfie, was classified as having severe dementia, but according to the 'clinical judgment' of the lead RN, he was considered an appropriate participant, and was invited to join the study. It was revealed through this study, that the clinical judgment based on knowing the PWD well, was a more accurate assessment of cognitive functioning, than the MMSE2:BV alone. This finding lends itself to future research.

The majority of participants (six) were living with four or more comorbidities and taking associated medication. There is robust evidence for a causal association with dementia and hypertension in midlife and diabetes across the life course (Prince et al., 2014). All but one participant were prescribed associated medications for pre-existing comorbid conditions (range 3-12). Six were being treated for hypertension and one for 
diabetes, along with other comorbidities not yet linked with dementia. Seven participants were prescribed associated polypharmacy of five or more regular medications (Lau et al., 2010). Results from a study of 7135 newly diagnosed patients with dementia found that dementia increased steadily with age, and was positively correlated with the number of medications used (Lai et al., 2012). Three participants with AD had been prescribed the acetylcholinesterase inhibitor (Donepezil), which had been commenced at the time of diagnosis and continued. One had been taking donepezil for at least three years and the other two for at least five years, suggesting a much longer duration than in the population based study in Taiwan where the average duration for acetylcholinesterase inhibitor administration was around 14 months (Sun et al., 2008). Two other participants with AD had never been prescribed donepezil or any other acetylcholinesterase inhibitor or memantine a N-methyl-D-aspartate (NMDA) partial antagonist medications, which have been found to provide some symptomatic relief by slowing down cognitive decline and loss of independence in moderate dementia of AD type (Birks, 2006; Brickell, 2012; McShane et al. 2006; NICE-SCIE, 2006/2015).

The majority of participants (eight) were prescribed mood-altering medications, either antidepressants (Joy, Bill, Clara, Alana), anxiolytics (Clara, Alfie), benzodiazepine for agitation (Alfie), or antipsychotics (Iris, Bill, Maya, Risa). Two of these medications (amitriptyline and the benzodiazepine - diazepam) are not first line treatment or should be avoided in dementia if possible (Bpac ${ }^{\mathrm{nz}}, 2013$; Small et al., 1997). Three medications (risperidone, quetiapine, prochlorperazine maleate) should be used with extreme caution in dementia, following a "start low, go slow" adage (Bpac ${ }^{\mathrm{nz}}, 2013$, p. 54). It is possible that the acetylcholinesterase inhibitor donepezil and mood-altering drugs will have influenced the findings of this study.

\subsection{Engagement with computer-assisted exergaming with Able-X}

Participants engaged in a non-pharmacological psychosocial activity of computerassisted exergaming with Able-X. All participants had an opportunity to play 12 times. Table 33 shows the engagement varied between the first two participants (Joy, Iris) and the remaining eight participants. Seven participants played solo and three played some gaming sessions in pairs or groups of three. Thomas' wife was always present when he was gaming and, although she did not participate in the study, she did occasionally play (see 5.5.3.5). By engaging in and continuing with this activity, each participant 
demonstrated energy for exergaming and his or her ability to have fun. Astell (2004) and Boulay et al. (2011) found similar results, during cognitive stimulation utilising computerassisted multi-sensory interfaces with PWD.

Table 33. Frequency of gaming with Able-X

\begin{tabular}{|l|r|r|r|r|r|r|r|r|}
\hline \multirow{1}{*}{ Name } & \multicolumn{9}{|c|}{ Game played \& frequency of play } & \multicolumn{1}{c|}{$\begin{array}{l}\text { Session Type \& } \\
\text { No. }\end{array}$} \\
\cline { 2 - 10 } & Circuit & Target & $\begin{array}{l}\text { Mosquito } \\
\text { Splat }\end{array}$ & $\begin{array}{l}\text { Butterfly } \\
\text { Catch }\end{array}$ & Popaloon & $\begin{array}{l}\text { Gaming } \\
\text { Sessions }\end{array}$ & Solo & Group \\
\hline Joy & 10 & 7 & 6 & 1 & 3 & 10 & 10 & 0 \\
\hline Iris & 6 & 8 & 4 & 4 & 5 & 8 & 8 & 0 \\
\hline Bill & 12 & 12 & 12 & 12 & 12 & 12 & 7 & 5 \\
\hline Thoma & 12 & 12 & 12 & 11 & 12 & 12 & 4 & 8 \\
\hline Maya & 12 & 12 & 12 & 12 & 12 & 12 & 6 & 6 \\
\hline Risa & 12 & 12 & 12 & 10 & 12 & 12 & 12 & 0 \\
\hline Clara & 9 & 9 & 9 & 9 & 9 & 9 & 9 & 0 \\
\hline Alfie & 13 & 13 & 11 & 11 & 11 & 11 & 11 & 0 \\
\hline Alana & 12 & 12 & 12 & 12 & 12 & 12 & 12 & 0 \\
\hline Florence & 12 & 12 & 12 & 12 & 2 & 12 & 12 & 0 \\
\hline
\end{tabular}

Being engaged in enjoyable and purposeful social occupation is one of the cornerstones of person-centred care (Brooker, 2004; Cooke et al., 2010; Dewing, 2008; Kitwood, 1993a, b, 1997; Morhardt \& Spira, 2013; Passalacqua \& Harwood, 2012; Røsvik et al., 2011). Additionally, social occupation through cognitive oriented interventions has been found to be significantly beneficial to aspects of QOL and wellbeing in PWD (Galea et al., 2016; Oyedobe \& Parveen, 2016; Spector et al., 2003; Woods et al., 2012).

\subsection{Theoretical applications to five emergent themes}

\subsubsection{Theme 1. Sentient interaction with the social gaming environment}

Before gaming could commence, participants were required to relocate from one environment to another (the gaming room). Some participants could apprehend and convey meaning through embodied sentient interaction with the environment (Kontos, 2004), which enabled them to become intentionally bodily agential (Kontos, 2004). For example, "when [Joy] is waiting for you she says, 'I've got an appointment", (DT:4/2:I:1104. "[Bill] gets quite anxious [because] he wants to play...He gets shirty because the others are in there on it [computer and exergames]" (NOK:I:11/8:568), and "[Florence] is looking for you. I might walk past...or one of her favourite caregivers, but she's not looking for them, she's looking for you" (RN:I:21/9:174). Joy, Bill, and Florence 
had the agency to draw on cues through sentient interaction with the environment, which connected them to gaming remembrance.

The provision of contextual cues, are recognised as beneficial EL strategies in the rehabilitation of PWD (Ballard et al., 2011; de Werd et al., 2013, 2015; Terrace, 1963; Sidman, 2010; Thivierge et al., 2008; van Tilborg et al., 2011). However, in a different environmental context in which I was not present as a contextual cue, Joy and Florence were unable to recall gaming, "I was saying to my cousin what Mum [Joy] was doing, it was gone, she knew nothing about the computer" (NOK:I:7/2:472). Likewise, "I'll say to her [Florence] what has she been doing and she'll say 'nothing' or that she 'can't remember' and then I'll ask her if she'd played any games, 'What games?' The computer games, 'Oh, yes, yes' she says. (NOK:I:6/10:647). Bill was the only participant to remember computer-assisted exergaming without me being present. This finding reiterates the importance of leaving contextual cues in the environment, to facilitate communication that is more balanced and reminiscence between PWD and others. Astell (2004) makes similar recommendations.

Slightly less sentient to the environmental cues (the arrival of me and my equipment), the remaining seven participants knew I was familiar to them, but they were unable to immediately make an implicit connection to exergaming or me. However, each of them exchanged greetings and pleasantries, which demonstrated their embodied intentional socio-cultural relationality and expressions of social and cultural etiquette, through implicit and spontaneous actions and behaviour (Kontos, 2004). Although I was familiar to each of the participants, in moments like these, they could not recall who I was or why I was there. In this situation, it is important that errors are not made by participants trying to guess who I am and what my intentions are (Evans et al., 2004). The probability of this eventuality can be anticipated and managed through research methods. In this study, procedural steps 1-5 (Table 10) facilitated a connection to sentient interaction with the exergaming gaming environment. This procedure introduced consistency, routine, and familiarity, which is known to create cognitive and emotional responses such as comfort and prediction (Son et al., 2002). Moreover, errors are reduced through creating an enriched therapeutic environment (Yamaguchi et al., 2010). Processes such as these are described as rigid and challenged by Sandelowski (2010), who advocates for more fluid, organic methods. Conversely, by being attentive to methods and procedures, the 
therapist/researcher can assure the integrity of the therapeutic environment and the intervention, in so doing, ensure that the ethical interest of doing no harm to PWD is observed (Drury, Francis \& Chapman, 2007).

\subsubsection{Theme 2. Initiation and gaming advancement in partnership}

All participants required initiation assistance to become gaming active at the start of each gaming session, and to varying degrees, during and between the exergames.

\subsubsection{Game shy}

Eight participants had no recall of gaming previously and two (Bill, Alfie), who could recall gaming, could not self-initiate or advance without help. I identified and named these phases of gaming inaction 'game shy', which I describe as gaming phases where participants are unable to self-initiate without assistance resulting in gaming 'inertia'. Gaming inertia can be anticipated and prevented by reintroducing the game at every session and before each game, in line with the principle and strategies of EL (Ballard et al., 2011; de Werd et al., 2013, 2015). Although game shy, all participants expressed socio-cultural etiquette, by being gracious, polite and helpful, in affording me the time and opportunity to introduce the project and myself and to obtain assent for gaming. Reciprocal respectful relationships between therapists and participants have shown a beneficial effect when communicating with PWD (Astell, 2004, 2009, 2010; Boulay et al., 2011). Furthermore, empathetic verbal and non-verbal communication has been shown to enhance the effects of interventions with this vulnerable group (Astell, 2004, 2010; Kawashima et al., 2005; Woods et al., 2012).

Joy, Risa, Clara, and Florence employed good verbal skills to seek assistance during gaming to enable advancement and success, which demonstrated intentional bodily agency and sentient interaction with the social exergaming environment. In contrast, Iris and Thomas used less probing and more abbreviated language to make sense of the gaming environment. For example, I asked Iris if she was ready to play, she replied, "That's a very interesting question, do you know?” (FN:16/1:930). In responding in this way, Iris was using what her NOK calls "cocktail chatter" (FN:16/1:931) to generate additional clues $^{37}$ to connect her to the social gaming environment, and in so doing demonstrates

\footnotetext{
${ }^{37}$ Clue: Something you hear or find that helps you answer a question or mystery.
} 
embodied socio-cultural relationality (Kontos, 2004). Kontos (2003) gives a similar example where she draws on 'Elegy for Iris ${ }^{38}$ to demonstrate embodied selfhood from an embodied socio-cultural source. She describes how Iris Murdoch could greet the postman at the door and call for her husband to attend to him despite her ability to communicate having "dwindled and faltered and almost ceased" (p. 167). However, Iris' social grace was still present and implicitly spontaneous.

Similarly, Alana and Maya operated in passive, but equally socially graceful ways. They sat quietly and waited for me to initiate them, as if waiting for their turn. In so doing, they demonstrated embodied expressions of social-cultural etiquette to be polite and personable, revealing inherent mastery of a common code that enabled them to apprehend the situation and convey meaning (Kontos, 2004). In the social time and space of computer-assisted exergaming we were all aware of each other's role and responsibilities for operating (Bourdieu, 1977, 1990), in that I knew that Alana and Maya were waiting to be initiated, and they were respectful of our relationship to wait patiently for me to take the positive action of gaming initiation. Similar findings were alluded to by Kontos (2004) when she describes how the study participant with the most severe cognitive impairment always said "thank-you" when her private sitter wiped food from her mouth or chin, and by Terrace (1963), with animal subjects who learned to discriminate without errors and quietly waited for the next positive prompt or cue to appear.

In contrast, Bill and Alfie were men of few words, as both had expressive dysphasia. They used intentional bodily gestures (Kontos, 2004) to express themselves such as a nod of the head, a shrug of the shoulders (B:VL:19a/8:14:57), or pointing at the exergaming screen (VL:10/9:00:00). They also used intentional bodily agency of verbal interjections to express themselves during gaming. For example, Bill would interject with "nooo, ooh, aah and yeah" (VL:13/8:48:00) and Alfie used, "aah, ooh, mm, aw, hmm" (VN:10/9:416). The meaning of gestures and interjections was further qualified through adjunct intentional bodily gestures: general body language, facial expressions, and paralanguage: sighs, gasp, throat clearing, pitch of voice, volume, intonation, pauses.

\footnotetext{
${ }^{38}$ Elegy for Iris is a book about Dame Iris Murdoch (Dame of the British Empire), an influential Irish philosopher, and novelist (1919-1999). The book, written by her husband John Bayley in 1999, describes life with Iris following her diagnosis of AD until her death.
} 
These findings are similar to those of Kontos (2004) when she describes the dining room conversation between Abe and Anna.

\subsubsection{Group gaming and 'gaming by association'}

Three participants (Maya, Bill, Thomas) played in pairs or in a group of three, plus one observer. If Thomas was present so was his wife. Group sessions provided feelings of belonging and opportunities for social interaction, similar to the finding of the music groups conducted by Boulay et al. (2011), where human contact was found to be an important factor. All group participants had the embodied capability to draw on their intentional bodily agency, expressions of socio-cultural etiquette and intentional sociocultural relationality within the computer-assisted exergaming field. This was clearly demonstrated in the group-play situations. Maya, the quietest and most shy of all gamers needed a chaperone for the first three solo playing sessions. However, in the group situation she was empowered to dismiss her chaperone as she sentiently interacted with a social environment in which she readily recognised familiar faces and therefore was more at ease. Bill and Thomas were supportive towards Maya and each other, therefore reducing the assistance required from me in group situations. It is known that self-submergence in the real world of research poses physical and emotional risks for both participants and the researcher (Dickson-Swift et al., 2006, 2007; Wray et al., 2007). By working in groups, these risks of emotional stress were reduced, for both Maya and myself.

A commonality across the group was the obvious enjoyment of engaged game watchers. I identified and named these gaming moments as 'gaming by association', where all involved are pleasured through actively gaming or actively supporting another gamer (VL:19a/8:01:15:58, 01:27:40). These findings are similar to the music group work of Cooke et al. (2010). All group members were also supportive of each other. Bill and Thomas often use intentional gestural agency as they swapped admiring and respectful glances and nods as they enjoyed each other's gaming success (VL:19a/8:14:57). By the last week of gaming, Bill was imitating Thomas by using the same words to encourage gaming success in Maya, such as “up, up” and “down, down” (VN:19a/8:2750), doubling his daily vocabulary and providing encouragement to others. Similarly, Thomas began imitating and responding to Bill by using some of Bill's gaming interjections, including "no, no [or] ah, ah" (VN:18/7:3007), inferring that, between them, they had mastered a 
coded communication system that enabled them to apprehend and convey meaning, similar to findings revealed by Kontos (2004) in her example of Abe and Anna.

All participants, irrespective of their stage of dementia, could communicate using words, interjections, body language, or gestures. There is supporting literature that demonstrates that CST using computers (Astell et al., 2004, 2009, 2010), or general cognitive-oriented therapies (Kawashima et al., 2005; Woods et al., 2012) can improve communication with PWD, particularly in the advanced stages of disease where communication may be less comprehensible. However, at this stage the purpose of communication is not to exchange information, but to exchange affection and empathy (Yamaguchi et al., 2010), both of which are demonstrated in the examples above.

A difference across group members was that Thomas was the only member to acquire intentional bodily agency to self-initiate. This was only possible if the transition from one player to the next was within 30 seconds, and the game was the same. The group setting provided opportunities to hear gaming instructions several times, during which there was a window of time in which self-initiation was possible for Thomas. In these moments, Thomas was intuitively drawing on EL cues, through sentient interaction with the environment, such as imitation and repetition. Similar EL strategies were used by van Tilborg et al. (2011).

\subsubsection{Repeated success of easy gaming}

A commonality across all participants was their reluctance to advance from the simplest gaming level to a more challenging level. I identified and named this gaming preference 'repeated success of easy gaming', which I describe as continued repetitive gaming at the most basic level, being preferred to the risk of not being successful at a more challenging level - a strategy that was preferred by all participants. On reflection, I considered that once gaming initiation was self-empowered, reluctance to game at a more challenging level might dissipate. This was the case with Alfie, who was self-initiating by session six, and may well have eventuated for other participants in the longer term. However, it is known that emotional responses such as comfort and prediction are the benefits of consistency, routine and familiarity (Son et al., 2002), and by facilitating repeated success of easy gaming, where errors are minimised, the principle of EL is achieved. Additionally, environments can be enriched through the implementation of EL 
approaches with PWD, as demonstrated by the findings of others (Clare et al., 2000; Clare \& Wilson, 2004; de Werd et al., 2013, 2015; Ferland et al., 2013; Jones \& Eayrs, 1992; Laffan et al., 2010; Metzler-Baddeley \& Snowden, 2005; Schmitz et al, 2014; Thivierge et al., 2008).

\subsubsection{Theme 3. Level of gaming intentionality: Influences}

\subsubsection{Game able}

Once initiated and gaming, of the five participants who remembered playing the games previously, four (Bill, Thomas, Clara, Alfie) achieved gaming independence within the first session of each game. They demonstrated sentient interaction with the computerassisted exergaming environment by intuitively expanding their body schema (MerleauPonty 2014) to incorporate the Able-X controller. Moreover, they used intentional bodily actions to purposefully and independently game. All four participants had used a computer previously, suggesting that they were also drawing on a second source of embodied selfhood, intentional socio-cultural relationality for operating in the field of computer use. Their previous experience enabled them to draw on a source of spontaneous implicit precognitive bodily know-how of how to use a computer that was evoked in response to initiation and engagement in the activity, rather than contemplation and reflection on the activity. I identified and named this phase of gaming as 'game able', a phase where participants play with independent agency, with rare or minimal assistance.

\subsubsection{Game enabled}

Another participant (Risa) also had previous experience of using a computer. However, unlike the others, she was initially unable to evoke her embodied agency for operating in the field of computer use. This was evident by the frequency of verbal support she required during gaming (every 10-20 seconds). However, verbal support requirements diminished over the gaming period and by the final session, she had an episode of independent gaming that lasted for four minutes, implying that over time she was able to reconnect with her intentional socio-cultural relationality of computer use. An alternative explanation is that Risa had relearned how to use the computer interface during the previous gaming sessions, which were influenced and bounded by the theoretical principle of EL (Ballard et al., 2011; de Werd et al., 2013, 2015; Terrace, 1963). It is difficult for PWD to remember or correct errors made when trying to learn or re-learn (Baddeley \& 
Wilson, 1994; Clare et al., 2000; Evans et al., 2004; Jokel \& Anderson, 2012; Jokel et al., 2010), therefore learning is more efficient if errors can be avoided during the learning process (Evans et al., 2004; Sidman, 2010; Terrace, 1963). In this study, I did all that was possible to implement the principle and strategies of EL, through my multiple roles.

Participants Joy, Iris, Maya, Alana, and Florence required a similar level of near constant verbal instructions to Risa to remain actively gaming. They had no recollection of gaming previously so each session was as if it was the first, but, like Risa, they could game in partnership with me, whilst following contemporaneous verbal instruction, during which they expanded their body schema to incorporate the Able-X controller, of which they had sole charge. In so doing, they demonstrated sentient interaction with the computer-assisted exergaming environment. I identified and named this level of gaming 'game enabled', as each participant was gaming with intentional bodily agency, albeit in partnership with me. There is evidence that being socially relational within a partnership of respectful communication creates an enriched environment that can enhance the benefits of interventional therapies (Astell, 2004; Yamaguchi et al., 2010).

\subsubsection{The influence of non-gamers in the gaming environment}

On a number of occasions, additional non-gamers were present in the exergaming environment, which could affect environmental ambience and energy. For example, when Bill's wife and grandson (Charlie) were present his gaming was at its most animated (VN:19/8:2676) and the ambience and energy levels were enriched and all present benefitted by association (VL:12/8: 30:00). Similar benefits of intergenerational enrichment programmes are reported by Galbraith et al. (2015) and George and Whitehouse (2011). Conversely, on two occasions Maya had a guest in the gaming room and the guest would occasionally express his opinions non-verbally with a gestural shake of the head (VL:20/8:09:00) or paralanguage such as huffing and puffing (VL:20/8:21:00). On one occasion, the guest tried to assist Maya by steadying her hands, which interrupted her independent and intentional gaming agency; she stopped gaming, dropped her hand, relinquished the controller, and looked away from the screen and away from the guest. The expression on her face was calm and accepting, her body language inferring that both the guest and the controller were no longer sharing the same space that she occupied. (VL:20/8:25:05). 
The actions and behaviour of the guest in the example above demonstrate what Kitwood (1990, 1993b, 1997) calls malignant social psychology, a term he used to describe the undermining of PWD through disempowerment, labelling and invalidation. Also demonstrated are the risks associated with the paradoxical processes inherent in relationships that can exist between PWD and others who know them well, which can include anger and frustration on the part of family and/or close relatives (Greenwood et al., 2016; Mittelman et al., 2004; Røsvik et al., 2011, 2013). However, Maya could respond to this situation by drawing on both types of embodied selfhood and intentionally disengaged with dignity. Being respectful and relational extends beyond the people involved in the interaction, to include the interventional technology and the environment.

\subsubsection{Positive regard and the impact of positive on-screen messages}

Positive on-screen messages and encouragement were integrated into the exergames. The Target game provided the prompts excellent, good and well done, whereas the games Mosquito Splat and Butterfly Catch communicated positive messages through noise and scores rather than on-screen words, all of which were catalysts for gaming motivation, stimulation, fun, and reminiscence. For five participants (Joy, Iris, Alana, Risa, Florence), positive messages, and gaming success facilitated reminiscence about cleverness or capability. Joy often reminisced about hearing her father say that her older sister was clever and she (Joy) was determined. Joy was motivated by this to prove that she was not only determined but also clever. For Iris, the success and fun of gaming reminded her of her "clever daughter... and [late] clever husband" (VN:28/11846), and on one occasion, in the flush of gaming success, she talked directly to the Able-X controller saying, "I'm very clever, you love me don't you?" (VN:23/3:1624). In addition, the fun of gaming connected Iris to her mum with whom she had "lots of fun" (VN:18/1:1825). Alana, was transported back to her "school days where they always encouraged [her] to do more" (VN:29/9:1690), and the artist, Risa, reminisced how the Circuit path traces reminded her of the "the movement of an artist arm and hand", as she demonstrated embodied selfhood through intentional gestural arm movements (VL:12/8:03:55). Moreover, the positive musical sounds of gaming linked her to memories of when she used to "sing at weddings" (VL:29/7:5:00). Florence was reminded of something that her Mum said, “I wasn't very clever, and Mum said it was a waste of money sending me [to school] (R:I:9/9:1197). Although Florence did not have dementia when her mother made these remarks, her mother's voice continues to be heard in the time and place occupied by 
Florence. However, when Florence was rewarded with positive on-screen scores she often said, "I can't believe it [smiling and laughing]" (VN:10/9:1722). "I no longer feel stupid (FN:1/10:620, 626).

In the above examples, each participant was intentionally embodied by their sentient interactions with the exergaming environment. Furthermore, each drew on intentional socio-cultural relationality that implicitly enabled them to reconnect to memories through reminiscence, and intentional bodily gestures. Stimulation generated reminiscence was not unexpected. It is known that reminiscence therapy contributes to improved sense of self and confirmation of continuity of self. In the case of Florence, although her reminiscences were negative memories, she could reconcile herself in the moments of computer-assisted exergaming as "no longer feel[ing] stupid", which demonstrated her ability to re-construct self through re-appraisal of self in a given time and space (Astell et al., 2004; Millett, 2011; Pearce et al., 2002). In addition, allowing the time for PWD to talk in the here and now (an EL strategy that does not require remembering previous conversations), control of the conversation is transferred to the PWD (Astell et al., 2004). In so doing, the person is empowered, validated, valued and recognised for their uniqueness, regardless of their cognitive ability (Kitwood, 1997; Passalacqua \& Harwood, 2012; Røsvik et al., 2011, 2013).

Five participants exhibited embodied selfhood by drawing on sentient interaction with the social gaming environment, through bodily movements and gestures. Clara, Thomas, Alfie, Bill, and Maya revealed their competitive spirit in the light of the positive messages and contemporaneous gaming scores. For Clara, the on-screen feedback made her "feel awake" (VN:28/7:2250) and the positive scores "tell me I have good concentration [and] I have control of my brain", as she gestured her hands to her head. (VN:30/7:2359). She also asked how she compared to others of a similar age, drawing on a second source of embodied selfhood, intentional socio-cultural relationality through habitual self-comparison. Alfie could not quite believe how well he was doing and habitually insisted on double checking his scores, which he learnt to do himself (LV:25/9:09:00).

For Thomas, positive messages would trigger witty repartee and gestures, drawing upon his habitual sense of humour, and his powers of gesticulation for example centring 
his tie, to celebrate a good score (VN:11/8:2671). For Bill, a very private man of few words, positive on-screen messages and positive regard from others made him smile and laugh (VN:19/8:2785), interject (VN:18/7:3007) and use the paralanguage of excited gaming noises (VN:13/8:2620). However, it was the positive regard and affection he received from his wife/NOK and grandson, that made him physically wakeful, lucid and more vital in gaming moments (VL:11/8:5:51). Maya, a lady of few words, used ocluesics $^{39}$ through searching for validation in the eyes and faces of others through eye related gesturing, such as: gazing, and trying to make eye contact, and frequent glances, as if seeking proof, over and above the positive on-screen messages, that she had reason to be pleased and proud of herself (VL:19/8:49:10).

All participants in the above two sections demonstrated one or more QOL indicators, which were evoked by the positive regard of others and from the positive onscreen messages, including expressions of wakefulness, lucidity, alertness, sociability, relationality, excitement, humour, joy, laugher, happiness and smiling. There are reports from other studies of self-reported perceived enjoyment from computer-assisted interventions for PWD (Boulay et al., 2011), which according to MacPhillamy and Lewinsohn (1982) are often associated with QOL in this population group. Moreover, it is known that activation of the muscles related to smiling evokes a pleasant feeling as an automatic, rather than a cognitive reaction (Strack, Martin \& Stepper, 1988), and the intentional embodied gesture of smiling, is one of the final gestures to be lost (Auer \& Reisberg, 1997; Reisberg, 1988). However, automatic reactions are not the same as embodied intentionality. The former denies the intentional creative nature of embodied selfhood.

\subsubsection{Error starer-error inertia: The influence of negative on-screen feedback}

It was not unusual for intentional disengagement behaviour to be triggered by the in-built negative feedback in the Able-X exergaming suit. For example, too slow, try again, missed, try again was the negative feedback in the game Target. The games Mosquito Splat and Butterfly Catch communicated negative messages through negative noises and minus scores. Where positive regard and feedback was a catalyst for

\footnotetext{
${ }^{39}$ Oculesics, a subcategory of kinesics, is the study of eye movement, eye behaviour, gaze, and eye-related nonverbal communication.
} 
empowering intentional gaming agency and confidence, negative messages and noises did the reverse and resulted in gaming inertia.

Negative feedback is the antithesis of EL principle and strategies. As the negative feedback was built into the gaming technology and triggered by a gaming error, I managed each error event by over-talking it with praise and encouragement, which became physically and emotionally draining for me as a researcher, and posed emotional risks for both participants and researcher. Emotional exhaustion is not uncommon in qualitative research (Dickson-Swift et al., 2006, 2007; Wray et al., 2007). Failure on my part to talk over, or reassure and encourage through negative feedback gaming moments resulted in a startled effect that momentarily disrupted gaming and triggered a period of gaming inertia. This period was usually followed by a run of errors in the game that hampered progress. I identified and named these gaming phases as gestural 'error starer-error inertia'. Moments that were only revealed through the process of reviewing and transcribing the video footage in my researcher role. The concept was not obvious to me in my role as gaming partner as I was preoccupied with gaming. Negative feedback was a major disabler when gaming with Able-X for all participants other than Thomas and Alfie, who rarely encountered it.

Following error starer-error inertia moments, in response to negative feedback, Joy and Iris developed aversive behaviour and intentionally disengaged from gaming and turned away from the screen. Others report similar aversive behaviour in relation to the activity of facilitating eating and drinking with PWD (Pasman, The, Onwuteaka-Philipsen, van der Wal, \& Ribbe, 2003; Norberg \& Hirschfeld, 1987; Norberg, Backstrom, Athlin, $\&$ Norberg, 1988). Behaviours not unlike the aversive behaviour in animal subjects (birds) which became agitated and would flap their wings, stamp on the floor and orientate themselves away from negative influences as reported by Terrace (1963).

Joy said, "It's not doing anything for me" (FN:17/2:268,273), following which she would have a period of gaming inertia or the game would be abandoned. She gestured by firstly turning away from the screen and then she disengaged ( $\mathrm{VN}: 15 / 4: 768)$. Iris would lose focus and generate an aversive response such as, "no, it's not my game”, following which she would abandon the controller and disengage (VN:23/1:1675). Clara (VL:13/8:07:00, 09:50) and Alana behaved similarly but did not disengage. Alana said 
the negative feedback makes her think that she is doing something wrong (VL:1/10:33:35), or make her feel insecure enough to ask, "am I doing it right?” (VN:1/10:1740). Bill very rarely received negative messages, but when he did, his demeanour became gesturally agitated and annoyed (VL:13/8:28:00) and similarly for Florence the distraction was evident by a gestural look of disheartenment etched on her face, or she verbally and bodily expressed frustration by berating herself (VL:18/9:10:00). For the above participants, momentary oppression of intentional bodily movement was the result of sentient interaction with the negative feedback from the Able-X suite of games. However, once the negative feedback stopped, embodied selfhood to game re-emerged.

\subsubsection{Influence on gaming of the Able-X controller}

A source of lack of gaming progress for some participants was related to the usability of the Able-X hand-held controller. The primary reason was related to the drift associated with the controller, which tended to drift away from the centre of the gaming screen. For example, the player may be pointing the controller to the centre screen, but the on-screen representation of the hand-held controller may be several centimetres off the centre point, requiring the player to move the controller until the on-screen representation is in the centre spot, or press a button to re-centre the controller. Although I tried to empower participants by teaching them how to re-centre the controller, I found that drawing attention to the many buttons on the device was counterproductive, as once noticed, the buttons and trigger became the focus for some participants. Similar problems utilising hand-held devices were found by Boulay et al. (2011). However, seven participants (Joy, Bill, Thomas, Maya, Risa, Clara, Alfie) intuitively, over time adapted their gaming technique to accommodate the drift associated with the Able-X hand-held controller, on average by gaming session six. This exemplifies how one's embodied self can dilate to accommodate instruments by embracing them. It also demonstrates how participants drew on their intentional bodily movement to connect with movement that was previously learned and understood by the body and incorporated it into its world (Merleau-Ponty, 2014), and in so doing, the movement participates with their body schema, generating effective intentional body movements. Until participants embodied the Able-X hand-held controller into their body schema, I assisted when I felt I needed to. For two participants, Joy and Bill, the intrusion was unwelcome, as evinced when I reached into their gaming moment to recalibrate the controller, they would gesture by pulling away from me (VN:21/1/1629). Conversely, other participants (Iris, Alana, Florence) were 
hampered by the drift of the Able-X hand-held controller and accepted help from me without complaint or question.

The second cause of interference with gaming progress for some participants was related to the trigger and buttons on the Able-X controller. The trigger was a dilemma for Iris, Bill, and Maya, who persistently wanted to use it on every game, and not just on the game in the sequence where it was required (Popaloon). These self-initiated actions could be due to the controller trigger and buttons resembling common operated tools which are ubiquitous throughout our lives, for example trigger tools like hair dryers, to spray bottles for cleaners or garden sprayers and hand power tools such as drills, and guns, most of which have buttons or levers to be pressed to activate. In which case, it can be asserted that all three participants were drawing on a source of embodied selfhood, of bodily agency of implicit know-how of how to use a trigger tool and buttons, all of which are based on previous experiences and emerged in response to initiation and engagement as opposed to contemplation and reflection (Kontos, 2004). In contrast, Florence found the trigger on the controller difficult to use in that she could press it but not release it again, which made the final game, Popaloon, impossible for her as it required index finger dexterity, a bodily movement that she could no longer master (VL:11/9:24:00,26:36).

\subsubsection{Theme 4. Hand-eye coordination}

The gaming efforts of all participants was embodied, intentional, functional, and purposeful. Each participant took advantage of the opportunity to repeat every game at the same level, which generated improved scores that were reward enough to keep participants motivated, engaged, and intentionally embodied in the exergaming environment. Once gaming, each participant advanced through the exergames with varying level of verbal prompting and occasional hands on assistive touch to recalibrate the Able-X hand-held controller; otherwise, all participants had independent charge of the hand-held controller. Each game built on the skills learned from previous games in the sequence. Gaming facilitation was informed by EL principles and strategies, similar to that described by others (Ballard et al., 2011; de Werd et al., 2013, 2015; Schmitz et al., 2014; Sidman, 2010; Terrace, 1963; Thivierge et al., 2008). 


\subsubsection{Quantitative findings: Hand-eye coordination}

The evidence presented in 5.5.4.1 and 5.5.4.2 addresses one of the objective criteria 1c of this study: to explore whether people age $\geq 65$ who are living with dementia, and are resident in care homes, achieve a physical function affect from using the Able-X. The Able-X computer-generated exergaming outputs (circuit graphs and scores) revealed improved hand-eye coordination for participants in this study. Table 34 shows the significant improvement between Circuit baseline and end-point scores for accuracy of hand/arm movement ( $\mathbf{p = 0 . 0 0 2})$, speed of hand/arm movement $(\mathbf{p = 0 . 0 4 3})$ and smoothness of hand/arm movement ( $\mathbf{p = 0 . 0 0 2}$ ) across all cases. In addition, significantly improved scores were discovered for Target $(\mathrm{p}=0.014)$, Mosquito splat $(\mathrm{p}=0.004)$, and Butterfly Catch $(\mathrm{p}=0.012)$. There was no significant improvement across the group for Popaloon $(\mathrm{p}=$ 0.570), the most difficult game in the sequence.

Table 34. Gaming outputs

\begin{tabular}{|c|c|c|c|c|}
\hline Measure & $\begin{array}{c}\text { Central } \\
\text { tendency }\end{array}$ & $\begin{array}{c}\text { Baseline } \\
n=10\end{array}$ & $\begin{array}{l}\text { After } \\
n=10\end{array}$ & $\begin{array}{c}\text { Wilcoxon } \\
\text { Signed Ranks } \\
\text { Exact Test }\end{array}$ \\
\hline $\begin{array}{l}\text { Circuit } \\
\text { Accuracy }\end{array}$ & $\begin{array}{l}\text { Mean (SD) } \\
\text { Median (IQR) } \\
\text { Range }\end{array}$ & $\begin{array}{r}74.7(16.7) \\
75.0(63.0-93.3) \\
46-95\end{array}$ & $\begin{array}{r}89.1(8.9) \\
92.00(84.3-95.5) \\
71-97\end{array}$ & $\begin{array}{l}\mathrm{Z}=-2.805 \\
\mathrm{p}=0.002\end{array}$ \\
\hline $\begin{array}{l}\text { Circuit } \\
\text { Speed }\end{array}$ & $\begin{array}{l}\text { Mean (SD) } \\
\text { Median (IQR) } \\
\text { Range }\end{array}$ & $\begin{array}{r}47.3(22.2) \\
45.5(25.5-61.0) \\
18-89 \\
\end{array}$ & $\begin{array}{r}56.2(19.6) \\
61.50(36.5-73.0) \\
31-83 \\
\end{array}$ & $\begin{aligned} Z & =-1.990 \\
p & =0.043\end{aligned}$ \\
\hline $\begin{array}{l}\text { Circuit } \\
\text { Smoothness }\end{array}$ & $\begin{array}{l}\text { Mean (SD) } \\
\text { Median (IQR) } \\
\text { Range }\end{array}$ & $\begin{array}{r}42.3(141) \\
44.0(28.0-57.0) \\
25-60\end{array}$ & $\begin{array}{r}60.1(11.4) \\
60.50(53.5-69.3) \\
37-75\end{array}$ & $\begin{aligned} \mathrm{Z} & =-2.807 \\
\mathrm{p} & =0.002\end{aligned}$ \\
\hline Target & $\begin{array}{l}\text { Mean (SD) } \\
\text { Median (IQR) } \\
\text { Range }\end{array}$ & $\begin{array}{r}2354.20(1100.35) \\
2346.0(1215.0-3397.5) \\
1010-4090\end{array}$ & $\begin{array}{r}3587.5(1250.6) \\
3295.0(2425.0-4593.8) \\
2250-5720\end{array}$ & $\begin{aligned} \mathrm{Z} & =-2.395, \\
\mathrm{p} & =0.014\end{aligned}$ \\
\hline $\begin{array}{l}\text { Mosquito } \\
\text { Splat }\end{array}$ & $\begin{array}{l}\text { Mean (SD) } \\
\text { Median (IQR) } \\
\text { Range }\end{array}$ & $\begin{array}{r}3933.0(2077.7) \\
3794.0(2013.5-5720.0) \\
1274-7152\end{array}$ & $\begin{array}{r}6951.3(2314.1) \\
6764.0(4903.0-8458.0) \\
4399-11427\end{array}$ & $\begin{array}{l}Z=-2.666 \\
p=0.004\end{array}$ \\
\hline $\begin{array}{l}\text { Butterfly } \\
\text { Catch }\end{array}$ & $\begin{array}{l}\text { Mean (SD) } \\
\text { Median (IQR) } \\
\text { Range }\end{array}$ & $\begin{array}{r}3843.7(1791.3) \\
3770.0(2250.5-5515.5) \\
1040-5972\end{array}$ & $\begin{array}{r}6834.3(3191.6) \\
7320.0(4621.5-9748.5) \\
1275-11169\end{array}$ & $\begin{array}{l}\mathrm{Z}=-2.429 \\
\mathrm{p}=0.012\end{array}$ \\
\hline Popaloon & $\begin{array}{l}\text { Mean (SD) } \\
\text { Median (IQR) } \\
\text { Range }\end{array}$ & $\begin{array}{r}84022.2(43722.0) \\
87600.0(35450.0- \\
125800.0) \\
26200-132250\end{array}$ & $\begin{array}{r}90100.0(43385.3) \\
89400.0(51225.0- \\
122600.0) \\
31650-167850\end{array}$ & $\begin{array}{l}Z=-0.652 \\
p=0.570\end{array}$ \\
\hline
\end{tabular}

This is the first study to report significantly improved hand-eye coordination in PWD, according to computer-generated exergaming outputs (circuit graphs and scores), resulting from the provision of cognitive rehabilitation utilising multi-sensory computerassisted exergame Able-X and EL strategies. However, Hijmans et al. (2011), utilised a 
similar movement based game controller (CyWee $\mathrm{Z}$ ) as an adjunct to regular rehabilitation with people with chronic stroke survivors, and reported significant improvement $(\mathrm{p}<0.001)$ of upper-limb movement compared to pre-intervention assessments as measured by the Fugl-Meyer Assessment upper limb section.

\subsubsection{Qualitative findings: Hand-eye coordination}

The qualitative impact of improved hand-eye coordination in the lived environment, manifested through enhanced functioning in some aspect of ADLs for seven participants. The similarities and differences were revealed through analysis of observations and subjective reports from proxy informants who knew each of the participants well. In the following examples, participants demonstrated enhanced intentional movements of the arms, hands and digits, which are the basis of many ADLs (Cohen-Mansfield et al., 2006; de Bruin et al., 2008; Verheij et al., 2012). Enhanced ADLs included 'self-grooming' (Iris, Clara, Maya, Thomas), enhanced 'toileting behaviour' (Alfie), 'self-feeding' and 'use of cutlery' (Alana), and enhanced independent mobility 'transporting and transferring self' (Florence). These examples evinced embodied selfhood that emanates from the body's natural intentionality of bodily movement. Furthermore, these examples infer that participants can connect with, or relearn movement that was previously learned and understood by the body and incorporate it into its world; a concept draws on other elements of embodiment such as implicit procedural memory, habitual experience of being in the world, and the ameliorative effects of neuroplasticity.

Hand-eye coordination is an important functional capacity that is associated with cognitive decline and highlights the importance of interventions that support independent functioning in an effort to delay the loss of independence of both IADLs and ADLs (Cohen-Mansfield et al., 2006; de Bruin et al., 2008; Verheij et al., 2012). Yamaguchi et al. (2010) assert that cognitive stimulation training and rehabilitation is "meaningless unless it serves to enhance... function in daily living" (p. 207). The above findings evince how improved hand-eye coordination resulted in enhanced functioning outside the gaming room according to proxy informants. This is the first study to report qualitative evidence of enhanced functioning of ADLs in PWD, according to proxy informants, resulting from the provision of cognitive rehabilitation utilising multi-sensory computer-assisted exergame Able-X and EL strategies. 
In addition, enhanced communication through social and relational activities was evidenced for nine out of ten participants, which demonstrated both sources of embodied selfhood, for example, Joy started writing letters after years of inactivity and reconnected with her friends. Bill, Thomas, and Maya, who gamed as a group, began to socialise together outside the gaming room. Bill, Clara, and Alfie became more wakeful and more communicative on gaming days. Alana and Florence communicated with more confidence resulting in new friendships for Alana, and Florence found her voice and became more assertive. Risa had enhanced interest to play the piano and sing several times a day, attracting large numbers of residents to her performances. Although the effects are varied across the cases in this study, similar beneficial effects for PWD are reported by Astell et al. (2004) and Passalacqua and Harwood (2012). This is the first study to report qualitative evidence of enhanced communication in PWD, according to proxy informants, resulting from the provision of cognitive rehabilitation utilising multi-sensory computer-assisted exergame Able-X and EL strategies.

\subsubsection{Theme 5. Aspects of QOL}

The evidence presented in 5.5.5.1 and 5.5.5.2 addresses the objective criteria 1a and $1 \mathrm{~b}$ : To explore whether people age $\geq 65$ who are living with dementia, and are resident in care homes, achieve a QOL affect from using Able-X (1a), or a cognitive affect from using Able-X (1b).

\subsubsection{Quantitative findings: $Q O L$}

Evidence of improved QOL was revealed from three additional outcome measures: Quality-of-life-AD scale (QOL-AD) (Logsdon, 1996); Cornell Scale for Depression in Dementia (CSDD) (Alexopoulos et al., 1988), and the Rating Anxiety in Dementia (RAID) (Shankar et al., 1999). The QOL-AD has a score range of 13-52, the higher the score the higher the assessed/perceived QOL (Logsdon, 1996). Findings from the QOL-AD scale inferred a significant difference on the scale in terms of improved QOL according to the lead RN ( $\mathrm{p}=0.004)$ and NOK ( $=0.023)$. However, Table 35 shows there was no selfreported significant difference on the scale $(\mathrm{p}=0.463)$. Similar results were found using the same tool on a similar population receiving an alternative CST by Spector et al. (2003). 
Table 35. Summary of findings from the QOL-AD outcome measure

\begin{tabular}{|l|l|r|r|r|}
\hline \multicolumn{1}{|c|}{ Measure } & \multicolumn{1}{|c|}{$\begin{array}{c}\text { Baseline } \\
\mathbf{n = 1 0}\end{array}$} & $\begin{array}{c}\text { End-point } \\
\mathbf{n = 1 0}\end{array}$ & $\begin{array}{c}\text { Wilcoxon Signed- } \\
\text { Rank Test } \\
\text { (exact test) }\end{array}$ \\
\hline $\begin{array}{l}\text { QOL-AD } \\
\text { (Self) }\end{array}$ & Median (IQR) & $42.00(39.75-45.00)$ & $41.00(39.00-47.50$ & $\mathrm{Z}=-0.822, \mathrm{p}=0.463$ \\
\hline QOL-AD & Median (IQR) & $38-46$ & $38-49$ & \\
(Lead RN) & Range & $38.50(35.75-43.50)$ & $45.50(43.75-47.00)$ & $\mathrm{Z}=-2.677, \mathrm{p}=0.004$ \\
\hline QOL-AD & Median (IQR) & $35.50(33.25-37.00)$ & $30-47$ & \\
(NOK) & Range & $25-39$ & $33.00(35.00-40.00)$ & $\mathrm{Z}=-2.313, \mathrm{p}=0.023$ \\
\hline
\end{tabular}

The biggest median difference in QOL was reported by the RN with median baseline score of 38.50 and end-point score of 45.50, an increase of seven quality of life points. The NOK reported 2.5 QOL point improvement and a baseline median score 35.50 and end-point score of 38.00. Participant self-scores did not significantly differ between baseline and end-point with a median score at baseline of 42, and 41 at end-point, a loss of one point. However, participants rated themselves as having a higher QOL baseline score than either the lead RN or NOK. The NOK perceiving the lowest QOL baseline score, although a significant improvement was revealed at end-point, this score was lower than the participants and the lead RNs baseline score. These results are not unusual. A recent study found female caregivers with a feeling of poor wellbeing were likely to be biased in their rating of others, and tended to overestimate functional disabilities and underestimate the QOL of patients (Jonas et al., 2011). Similar findings are revealed by others (Moyle et al., 2012; Spector \& Orrell, 2006; Winzelberg et al., 2005; Woods et al., 2012). Although improvement in QOL has been demonstrated by others in relation to the provision of cognitive interventions for PWD (Oyedobe \& Parveen, 2016; Spector et al., 2003; Woods et al., 2012), this is the first study to report significantly improved QOL in PWD, according to the global measure QOL-AD (proxies), resulting from the provision of cognitive rehabilitation utilising multi-sensory computer-assisted exergame Able-X and EL strategies.

Cognitive functioning is an indicator of QOL (Aguirre et al., 2010; Ballard, Khan, Clack, \& Corbett, 2011; Oyebode \& Parveen, 2016; Requenta et al.,2004; Tarraga et al., 2006; Woods, Aguirre, Spector, \& Orrell, 2012). The degree of cognitive decline for participants in my study was categorised as severe at baseline when assessed with the MMSE-2:BV cognitive measure (Folstein et al., 2010), with a score range of 1-9, median 5.5. At the end-point retest there were some differences in scores (range 3-13, median 6.00), but these differences were not significant $(Z=0.639, p=0.586)$. Participant MMSE- 
2:BV score increased for four, was maintained for two, and decreased for four. However, one participant (Joy) had an improved MMSE score of 4 points from 9 to 13 at end-point, positioning her at 1.5 point below her healthy peer group when her results are smoothed for age and educational attainment (Folstein et al., 2010). There was a slight beneficial change in cognition according to the MMSE-2:BV (but not significant), resulting from the provision of cognitive rehabilitation utilising multi-sensory computer-assisted exergame Able-X and EL strategies. It is known from a meta-analysis of the literature that evidence of effectiveness of computerised-cognitive interventions for people with $\mathrm{AD}$ has a weak to moderate effect size in their ability to improve cognitive function (Gelfand et al., 2014). Furthermore, skills and functioning can be mediated through cognitive rehabilitation (Woods et al., 2012).

Depression is the most common mental illness in adults and is associated with lower levels of QOL and high levels of disability in PWD (Moyle et al., 2012; Prince et al., 2014; Snowden et al., 2003). It remains unclear whether depression is a causal factor or a symptom of dementia (Prince et al., 2014). Findings from the CSDD revealed a difference on the scale in terms of reduced scores for seven participants, the range of scores was 0-6 baseline and 0-2 at end-point, however, this difference was not significant ${ }^{40}$ (Table 36).

Table 36. Summary of findings from the CSDD and RAID outcome measures

\begin{tabular}{|l|l|r|r|c|}
\hline Measure & & \multicolumn{1}{|c|}{$\begin{array}{c}\text { Baseline } \\
\mathbf{n = 1 0}\end{array}$} & $\begin{array}{c}\text { End-point } \\
\mathbf{n = 1 0}\end{array}$ & $\begin{array}{c}\text { Wilcoxon Signed-Rank } \\
\text { Test (exact test) }\end{array}$ \\
\hline CSDD & Median (IQR) & $2.00(0.75-3.00)$ & $0.50-(0.00-1.25)$ & Z $=-1.799, \mathrm{p}=0.094$ \\
& Range & $0-6$ & $0-2$ & \\
\hline RAID & Median (IQR) & $3.50(1.00-5.25)$ & $1.00(0.00-2.25)$ & $\mathrm{Z}=-2.555, \mathrm{p}=0.008$ \\
Scale & Range & $0-8$ & $0-5$ & \\
& & & & \\
\hline
\end{tabular}

One participant (Iris) score reduced by 5 points, the largest reduction across the group. However, a few days before the first assessment was completed, the participant experienced the death of a close friend, which may have affected the baseline score. One

\footnotetext{
40 Although a non-significant result was obtained this might be a type II error, and so in addition to the Wilcoxon Signed-Rank Test, a post hoc bootstrapped 95\% (percentile) confidence interval for the observed median change from baseline to the end-point of -1.5 was obtained based on 1000 samples $(0.0,2.0)$. Bootstrap analysis in $\mathrm{R}$ indicated that the chance of Type II error is high as the sample power is low at $41.2 \%$. To achieve $80 \%$ power, 20 individuals are required.
} 
participant (Joy) scored 2 on both occasions and for two participants (Risa, Maya) their scores increased from 0 to 1 and 0 to 2 . These results indicate that all participants were below the threshold of significant depressive symptoms (score of 8 or more) (Alexopoulos et al, 1988). In the study by Khan et al. (2016) that utilised computer-assisted exergaming with Able-X, for people with neurological presentations, alongside three other exergaming/gaming devices and sensory stimulation therapies revealed significant improvement at discharge when compared to controls in depression and stress subscales. However, the effect of the Able-X on these outcomes was not evaluated. This is the first study to report a beneficial (but not significant) effect on depression in PWD, according to the CSDD, resulting from the provision of cognitive rehabilitation utilising multisensory computer-assisted exergame Able-X and EL strategies.

One of several indicators of an underlying depression is anxiety. Absence of anxiety is considered an important aspect of QOL in PWD, although association between anxiety and dementia is not yet determined (Alexopoulos et al., 1988; Prince et al., 2014). The findings from RAID showed a statistically significant difference of $(\mathrm{p}=0.008)$ in levels of anxiety from baseline to end-point (Table 36). The range was 0-8 at baseline and 0-5 at end-point. One participant (Maya) scored zero on both occasions and another participant (Iris) scored one on both occasions. The results indicate that eight participants scored lower on the scale at end-point and all participants were below the threshold of significant clinical anxiety, which is indicated by a score of 11 or more (Shankar et al., 1999). This is the first study to report significantly improved anxiety levels in PWD, according to the RAID, resulting from the provision of cognitive rehabilitation utilising multi-sensory computer-assisted exergame Able-X and EL strategies.

\subsubsection{Qualitative findings: $Q O L$}

Behavioural and psychological symptoms of dementia (BPSD) is a term used for symptoms of disturbed perception, thought content, mood and behaviour frequently occurring in PWD (Bpac ${ }^{\mathrm{nz}}$, 2013; Perkins, 2013; Prince et al., 2014; Snowden et al., 2003). The psychological symptoms of depression and anxiety have been presented above. Other psychological symptoms include hallucinations, paranoia or other delusions and loss of emotional control (Bpac ${ }^{\mathrm{nz}}, 2013$; Perkins, 2013). Improvements in some symptoms of BPSD were observed in nine of the ten cases. 
Computer-assisted exergaming with Able-X appeared to have had a beneficial effect on behavioural symptoms of dementia for nine participants according to proxy observers and reporters who knew each participant well: less wandering/pacing/trying to escape (Joy, Iris, Bill, Thomas, Maya, Clara, Alana); less agitation in the late afternoon (Bill, Thomas); less restlessness with anxiety (Joy, Iris, Bill, Thomas, Maya); less apathy and inactivity (Iris, Bill, Clara, Alfie); less clinginess (Alana, Maya); less repeating of questions/repeated actions (Joy, Florence); less sleep disturbance (Bill, Clara, Alfie); less incontinence (Alfie); and less flirty/inappropriate behaviour (Alana). In each case, participants could demonstrate embodied selfhood through their improved intentional behaviour. Moreover, Alfie and Alana drew on their expressions of socio-cultural etiquette and intentional socio-cultural relationality to realign their behaviour, for operating within the environment of the residential unit. This is the first study to report qualitative evidence of beneficial effects on BPSD in PWD, according to proxy informants, resulting from the provision of cognitive rehabilitation utilising multi-sensory computer-assisted exergame Able-X and EL strategies. Similar findings of improved BPSD in PWD, following alternative cognitive oriented interventions were found by Kawashima et al. (2005) and Spector et al. (2000).

Also revealed was enhanced motivation and bodily intentionality to be social and engaged in 'psychosocial activities' within the lived environment. Joy, Bill, Thomas, Maya, Clara, Alfie, Alana, and Florence spent substantially more time in the psychosocial activity programme within the facilities than before computer-assisted exergaming. Risa increased her piano playing from once or twice a week, to several times a day, drawing on socio-cultural ways of being-in-the-world of music and song, which are implicitly primed through activity and implicit pre-cognitive bodily know-how, of how to play the piano without music and recite songs without the song sheets. Similarly, Iris started dressing up and attending intimate afternoon teas with her new 'friend', in so doing, she demonstrated social grace and inherent mastery of a common code of etiquette and good manners. This is the first study to report qualitative evidence of beneficial effects on motivation to reengage in psychosocial social activities, according to proxy informants, resulting from the provision of cognitive rehabilitation utilising multi-sensory computer-assisted exergame Able-X and EL strategies. Also, being social and relational is an indicator of QOL (Astell, 2004; Boulay et al. 2011; Hale et al., 2012; Logsdon, 1996; Smith et al., 2005, 2007; Woods et al., 2012). 
Objective criteria 2 was to explore if Able-X, originally developed for stroke survivors, can be reconceptualised for people with dementia. I allude to some elements of the Able-X prototype (2013) that require additional evaluation and possible redesign. However, I believe the Able-X (2013), originally developed for rehabilitation of chronic stroke survivors, can be reconceptualised for people with dementia. A more comprehensive account relating to the reconceptualization of the Able-X for people with dementia is reported elsewhere (Drury-Ruddlesden, deVries, Nelson, Kernohan, 2017).

\subsection{Chapter summary}

This chapter has described and critically examined the effects on QOL, including cognitive and physical functioning, of computer-assisted exergames (Able-X), as an adjunct to existing activities and treatments, for 10 people diagnosed with severe dementia, according to the MMSE-2:BV. To facilitate analysis, synthesis, and interpretation, I utilised the theoretical framework of embodied selfhood and other supporting literature to explore how the participants evince embodiment through intentionality of individualistic bodily movements and gestures, and through sentient affinity with the environment and socio-cultural ways of being-in-the-world. I also drew on, and critically applied the principle and strategies of EL and supporting literature to facilitate critical interpretation of my findings.

The findings reveal significant improvement in hand-eye coordination, QOL, and anxiety levels across the cohort. Moreover, there was a slight beneficial effect for depression and cognitive functioning but this was not significant. In addition, qualitative findings implied enhanced functioning of ADLs for seven participants, enhanced communication for nine participants, improved BPSD for nine participants, and enhanced motivation to re-engage in psychosocial activities for all 10 participants.

Through the theoretical and analytic lens of embodied selfhood (Kontos, 2003, 2004), and other supporting theories, I have critically interpreted how the participants in this study engaged with the world of computer-assisted exergaming with Able-X, within an enriched environment of EL. Furthermore, I have revealed how the benefits of this experience influenced the everyday lives of PWD, with a focus on answering my research question. In so doing, I have evinced how the embodied selfhood of each participant 
emanates from four pre-reflective sources: intentional bodily movement, gestures, and affinity with the environment, socio-cultural behaviours of the body, automatic bodily memory, and residual implicit (procedural memory). Sources that continue to interact with the world despite loss of cognitive functioning. Sources of embodiment that manifests as 'I can' in contrast to 'I think' (Merleau-Ponty, 2014). 


\section{CHAPTER SIX: CONCLUSIONS}

\subsection{Introduction}

This original research into computer-assisted exergaming with Able-X and EL strategies demonstrates a beneficial effect on global QOL and wellbeing of PWD. The research brought to the fore the importance of developing exergaming interventions that have ameliorative effects on the everyday abilities of PWD in an effort to delay loss of independence and in so doing improve QOL and functioning. The research demonstrates the application of the contemporary foundationalist lens of embodied selfhood described by Kontos, a lens through which to see, understand, interpret, and communicate the intentional and embodied actions of PWD. This new way of viewing PWD challenges the Cartesian mind-body dualism epistemology of Descartes and the symbolic interactionist stance taken by Kitwood. The findings demonstrate the importance of environmental enrichment and respectful therapeutic relationship, through the provision of cognitive rehabilitation within an environment bounded by EL strategies, informed by the seminal work of Terrace (1963), and the ethical and moral principles of Kitwood (1997). These findings demonstrate how people with the most severe dementia 'can do' and even learn new things. In so doing, the body draws on its very essence of implicit bodily memory and socio-cultural heritage that is primed and imbued within every fibre of the body, through the human process of being present in, and interacting with the world in any given time and space.

This chapter concludes this thesis. The chapter begins with a critique of the methodology and methods, where I justify my rationale for utilising case study, following which I summarise the key strengths and limitations of this study. I then make explicit how this research contributes to methodology, methods, and knowledge, by providing new insights and understandings related to the provision of computer-assisted exergaming with Able-X and EL strategies. An overview of the implications for practice and recommendations for future research is followed by a concluding statement. The chapter closes with a poem that embraces the key theoretical concepts that bind this thesis, and contains glimpses of the findings that emerge from this research.

\subsection{Methodological and method critique}

CS is both a methodology and a method that supports deconstruction and reconstruction of certain phenomenon, viewed through a variety of theoretical lenses. By 
using CS methodology this thesis provides moving and engaging impressionist 'stories' of embodied presence and intentional engagement in the activity of computer-assisted exergaming with Able-X. In each of the stories, I concretely hold up the voices of PWD and those closest to them, by recording them in the real and natural world in which they were situated. Through the stories, I constructed a biographical glimpse of each participant, and those with whom they are closest. As the stories developed, I wove in the fleeting moments of fieldwork that were captured over 62 hours of video recordings of exergaming with the participants, and 26 hours of audio recordings from people who knew the participants well. It is known that stories can be slanted, especially when generated from PWD and others closest to them, since each have different views of the world and of the people within it, because all live in a different time and space; the PWD live in a time and space of emotional embodied reality, while others, who walk alongside usually live in a time and space of cognitive reality. These differences are captured in the individual cases.

As a social pragmatist, I focused on addressing the research problem, rather than the purity of the philosophical approach. I needed to understand the change brought about by using Able $\mathrm{X}$ and how this change translated into the lived environment of each of the participants. This philosophical approach recognises that individuals seek understanding and subjective meaning from within the world in which they live. Meanings that are varied and lead the researcher to look for complexity of views to generate knowledge and if the approach allows, make use of quantitative statistical analysis to further validate qualitative findings, a known strategy to neutralise the weaknesses inherent in each data type. I realise that in parts this thesis may read like a mixed methods study, and I acknowledge that this could have been designed as such - but using a mixed methods approach would not have captured the individual 'stories' set out in first findings chapter. Both a strength and limitation of this CS is that it has produced comprehensive results that are close to dementia in the context of the real world in which the participants are situated, and through the process of triangulation of data sources gave richness to the study. The results may be less precise that those of pure science but they are sufficient.

The strength of this study is in the choice of an ontological approach of social pragmatism, combined with interpretive epistemology, within a collective convergent parallel CS methodological design, as the best research approach to elicit knowledge about 
how Able-X affects the QOL, cognitive functioning, and physical functioning in PWD. Although there are validated instruments available to measure QOL and other associated aspects, none of these in isolation or collectively would involve detailed exploration. In addition, there is a paucity of knowledge in the literature about how the improvements found, utilising validated instruments, translate back into the lived environment. Although a pluralistic approach is used in this study, it is weighted towards an interpretivist epistemology that makes use of quantitative evidence to test qualitative findings and offers new insights into emerging evidence on how cognitive rehabilitation through computerassisted exergaming with Able-X affects the QOL of PWD, and how these effects translate back to the lived environment.

Every study has biases and limitations. One of the limitation of this study is the utilisation of proxy ratings, which can be modulated by informant's variables, and therefore likely to be biased, which limits the usefulness of the data in such cases. To reduce this limitation, ratings were obtained from at least two sources who knew a participant well, and from the participants themselves, providing multiple sources of data on each aspect of the inquiry.

It might be argued that my tetrachotomous role could have influenced the findings of this study. However, while my roles may have impacted on the results of the study at the time of the research, I do not consider this to be a limitation as I only had an average of 18 hours of contact with each participant during this study, conducting three different activities: pre-and post-assessments (three hours), assent and mobilisation (three hours), and exergaming (twelve hours). The remaining fieldwork hours were utilised with other research activities that did not require direct contact with the participants. Furthermore, all but one of the participants could not remember my name or initially recall exergaming. In addition, other respondents noted changes between my visits, and more importantly, the changes remained long after I ceased interacting with the participants.

A second limitation is related to the potential for some of the beneficial findings to be related to the administration of pharmacological treatments. Three participants with $\mathrm{AD}$ had been prescribed the acetylcholinesterase inhibitor (Donepezil), which has been shown to have a beneficial effect on slowing cognitive decline and functioning in PWD. Furthermore, eight participants were taking mood-altering medications either: 
antidepressants (4), anxiolytics (2), Benzodiazepine for agitation (1), or antipsychotics (4). While it is possible that the acetylcholinesterase inhibitor, and mood-altering drugs could have influenced the findings of this study, many participants had been on the medications for a period of time, and in only one case (Bill) did the proxy rater (NOK) consider that the medication might have had an impact.

\subsection{Contribution to methodology and method}

This research contributes to CS methodology and methods of data collection and interpretation that embraces three key factors that were identified in the literature as having enhancer qualities in relation to the provision of cognitive oriented interventions for PWD. The literature revealed that an enriched environment, the role and behaviour of the therapist, and value, regard and respect given to the PWD as participants or partners in research, could be of benefit to interventional experience. In this CS design, all three elements were integrated into the approach, resulting in four very clear emergent roles for me: researcher, teacher, game partner, and study subject. Through these roles, I created an enriched environment, by applying the principle and strategies of EL, and ethical and moral enrichment, which are attended to through the absolute value and regard given to PWD, by interpretation and communication of their behaviour through the lens of embodied selfhood.

This emergent comprehensive design, requires the researcher carrying out these multiple roles to be consciously aware of the impact of his/her involvement in the whole research process, including their impact on participants and the intervention as he/she moves fluidly from one role to another. For example, if participants are 'game shy' or need to be 'game enabled', the researcher adopts the 'teacher role'. However, as soon as the participant becomes 'game able', the researcher is back in 'researcher role', observing the game and watching for negative influences that could disrupt gaming or cause 'errorstarer-error inertia', that would require a 'game partner', which positions the researcher alongside the participant as a 'study subject'. For the researcher to be partner and/or study subject, an alternative observer is required. This study utilised a video recorder as the 'persistent observer' to allow me to submerge myself in the real-world gaming experience. This methodological contribution not only has implications for how researchers engage in interventional studies with PWD, but also in other studies where the researcher has multiple roles. 
There are several audiences for these methodological and case study findings: academic colleagues, other professional colleagues, policy makers, industry representatives and funders of this study; all of whom will be interested in the relationship of the cases, the findings, how they relate to previous research and what they contribute to knowledge, research methods, and methodology. There is however another equally important audience, PWD and those who walk alongside them; they will have an interest in the case stories and how the stories relate to their own experiences of living with or supporting someone living with dementia. By presenting the cases in two separate chapters, I believe it allows all audiences to be satisfied.

\subsection{Contribution to knowledge}

This research makes a substantial contribution to knowledge, by providing foundational, in-depth knowledge about the effects of computer-assisted exergaming, utilising Able-X, and EL strategies, with PWD. It builds on the scarce clinical literature that focuses on Able-X and stroke rehabilitation, and other neurological conditions. Moreover, clinical research addressing computer-assisted interventions for PWD using Able-X was non-existent, prior to this research. Also unexplored was the value of facilitating Able-X within an 'enriched' environment that aims to reduce the risk of making 'errors' during the intervention process. This is the first study to report significant results utilising commonly used validated outcome measures that are supported by qualitative findings. These results demonstrate a significant improvement in QOL in PWD, as indicated by the global measure QOL-AD (proxies). Other findings relating to QOL include a beneficial (but not significant) effect on depression in PWD, according to CSDD scores; significantly improved anxiety levels in PWD according to the RAID scores; evidence of beneficial effects on BPSD in PWD and on motivation to re-engage in psychosocial social activities, according to proxy informants. The findings also evinced significant improvement in hand-eye coordination, which was supported by qualitative evidence of enhanced functioning of ADLs for seven participants, and enhanced communication for nine participants. In addition, there was a slight beneficial change in cognition, according to the MMSE-2:BV, but this was not significant.

This research reveals knowledge about the benefits of cognitive rehabilitation for PWD in the stage of 'severe' dementia according to the MMSE-2:BV. In so doing, this 
study contributes new knowledge to the very small literature base for this vulnerable group, as the majority of the literature focuses on research on people in the early stages of mild cognitive impairment or mild to moderate dementia. Also demonstrated is that the clinical judgment of the RNs and HCAs, based on knowing the PWD well, is a more accurate assessment of cognitive functioning, than the MMSE-2:BV alone. This was achieved by applying a more global measure (GDS/FAST staging system).

This research challenges the contemporary literature, which recommends that cognitive intervention therapy should be made available only to people with mild to moderate dementia. The findings demonstrate that cognitive rehabilitation through computer-assisted exergaming, utilising Able-X and EL strategies in later stages of dementia is beneficial. In addition, this study brings new understanding by challenging previous literature that argues that the principle and strategies of EL should be focused on simple cognitive tasks when intervening with PWD. Contrastingly, this study revealed that EL principle and strategies have much wider scope of applicability, including consideration at the design and implementation stages in all aspects in which PWD interact with the world.

This research revealed six original concepts that are indicative of the level of embodiment in relation to exergaming. These are game shy, game enabled, game able, gaming by association, repeated success of easy gaming, and error starer-error inertia. All participants in this study required initiation assistance to become gaming active. I conceptualised these phases of gaming inaction 'game shy' periods where participants required reintroduction to the technology at every session and before each game. Although game shy, each participant used pre-reflective sources of embodied selfhood to deal with gaming initiation, and once in the gaming room, all sources of embodied selfhood emanated as the participants were initiated to the Able-X hand-held controller and the computer-assisted exergames. One participant (Alfie) could self-initiate by the midway point (gaming session 6 of 12) and another (Thomas) used intentional bodily agency to self-initiate in the small group situation if the transition from one player to the next was within 30 seconds, and the game was the same. Once initiated five participants were 'game enabled' to game using the Able-X hand-held controller independently, with initial verbal prompting every 5 to 20 seconds in order to remain actively gaming. They had no recollection of gaming previously, so each session was as if it was the first. However, they 
could follow contemporaneous verbal instruction. Once initiated and gaming, five participants remembered playing the games before, and four of them achieved gaming independence within the first session of each game, and assistive input from me was minimal, they became 'game able'. They demonstrated sentient interaction with the computer-assisted exergaming social environment, by intuitively expanding their body schema to incorporate the Able-X hand-held controller and they used intentional bodily movement, to purposefully and independently game.

Once actively gaming, all participants were reluctant to advance from the simplest gaming level, preferring the reward of higher scores at the simplest level, rather than the challenge of a slightly harder game. I conceptualised these gaming moments as 'repeated success of easy gaming'. This concept fits with the principle and strategies of EL. The antithesis or EL principle and environmental enrichment was generated by the negative feedback from either the exergaming screen, difficulties with the hand-held controller Able-X, or other negative influences that occasionally found their way into the gaming environment, all of which triggered an embodied response that I called 'error starer-error inertia'. Moments of interrupted gaming 'error-inertia' that were brought about by negative influences that were heralded by the embodied gesture of staring at the screen, or at the negative influence, before disengaging from exergaming completely. Finally, the sixth concept is 'gaming by association'. One of the benefits of group gaming was the obvious enjoyment of all engaged game watchers, where all involved are pleasured through actively gaming in the company of others, or pleasured through actively supporting another gamer, resulting in all aspects of embodied selfhood being displayed. For example, two participants would often use gestural agency as they swapped admiring and respectful glances and nods as they enjoyed each other's gaming success, following which they started to emulate each other through an abbreviated language, inferring that they had mastered a coded communication system that enabled them to apprehend and convey meaning between each other.

The collection of six novel concepts described above lend themselves to other interventions with PWD not just exergaming. For example, Joy went from being 'shy' of writing letters for several months before reconnecting with this activity post-exergaming, becoming 'able' to write letters, without needing to be 'enabled', as she reconnected to a both sources of intentional embodied selfhood through a 'repeated success' of an activity 
that reconnecting to her social networks, therefore being pleasured 'by association'. In so doing, Joy did not encounter 'error-starer-error inertia' moments. Another example is related to IADLs. When Clara entered the establishment, she was 'able' to clean her room and make her bed. However, in her first few weeks of residence, these activities were routinely done by staff, creating a negative environmental influence, which created 'errorinertia', and the bed making and cleaning activity ceased as she became 'shy' of these activities. However, following exergaming Clara became more wakeful and 'enabled', and through the advocacy of her NOK, she reclaimed the cleaning and bed making activities. Following 'repeated success', Clara became 'able' to continue with these activities unassisted, which resulted in additional activities being allocated to her, including daily feeding of the cat and the fish. Both of which created pleasure 'by association', and ensuring a recognised role for Clara within the establishment.

This research advances the important theoretical knowledge of Kontos' original concept of 'embodied selfhood'. Kontos' work is well cited in the literature, although evidence of application and interpretation in the work of others was limited to one study, although Kontos continues to advance her embodied selfhood work. This study is the first robust application of Kontos' theoretical analytic framework in the real world of computer-assisted exergaming, using the methodological approach of social pragmatism, interpretive epistemology within a collective convergent parallel CS methodological approach, rather than through pure ethnographic observations that has been the case in the literature to date.

\subsection{Implications for practice}

There are a huge number of psychosocial interventions available for PWD. However, the evidence of benefit for most of them is yet to be determined. The beneficial evidence is strongest for cognitive stimulation therapy (CST), cognitive stimulation, and cognitive rehabilitation, particularly on cognitive functioning, daily functioning (ADLs) and QOL, similar to the findings reported in this research. However, these therapies are often not implemented outside research projects due to labour intensiveness of the approaches, which are yet to undergo cost-effectiveness evaluation. In this study, all the research sites (residential care settings) had multiple daily psychosocial activities, all of which were supervised by a diversional therapist (two sites), or a healthcare assistant (one site). Health providers should: 
1. Consider commissioning a cost-benefit analysis of the current psychosocial activities programme, to see if resources can be released for the provision of cognitive oriented therapies that have shown to have ameliorative benefit for PWD.

2. Be informed that computerised cognitive stimulation with older adults is more effective and less labour intensive than the more traditional approaches and likely more cost effective than other more labour intensive cognitive oriented approaches.

3. Consider the benefits of providing cognitive rehabilitation utilising computerassisted exergaming technology, which is portable, and could travel with PWD to alternative locations, including into hospital and or into the community or domestic setting, as long as there is computer access. This would contribute to the consistency and familiarity of the exergaming environment, and would provide continuance of beneficial rehabilitation at a time where their dementia is not the primary focus, for example during an acute episode of illness when hospitalisation or palliative support is required.

4. Provide education and training opportunities to all staff in care environments on the concepts and teachings of embodied selfhood after Kontos, as an alternative lens through which to see, understand, interpret, and communicate the intentional and embodied actions of PWD.

5. Provide education and training opportunities to all staff in the care environment on the principle and strategies of EL to create enriched environments in which PWD sentiently interact in their everyday lives, including the environments where PSI take place.

6. Consider engaging in future research activities and providing access to PWD in an effort to address stigma and discrimination, which is compounded by low numbers of PWD taking part in research activities. 


\subsection{Implications for industry}

This research explored and revealed that the computer-assisted exergame Able-X (2013) that was developed for the rehabilitation of stroke survivors could be reconceptualised for PWD. Due to commercial interests of Im-Able Ltd., these findings and recommendations are reported elsewhere.

\subsection{Recommendations for further research}

A number of key areas of future research relating to cognitive rehabilitation utilising computer-assisted Able-X arise from this study:

1. The current study principles should be used to develop a mixed methods study using a larger sample size, which would allow the results to be confirmed.

2. The Able-X (2013) could be reconceptualised for PWD, following which the study could be repeated to provide further insights into the ameliorative benefits of computer-assisted exergaming with Able-X (reconceptualised). In addition, the impact of hand-eye coordination and objective measurements of ADLs, and costeffectiveness evaluation would add value.

3. The current study could be replicated and the game partner (researcher) be replaced by an intergenerational other, a volunteer (s) known to the family, or caregivers known to the family and PWD.

4. An exploratory study would provide insights into culturally sensitive models of delivering cognitive rehabilitation interventions for PWD in other ethnic groups in NZ, including Māori, Pacific and Asian peoples.

\subsection{Future and potential publication}

The next stage of dissemination is to develop a series of lists on publication of this research. Work is underway on items 1 and 2.

1. Computer-assisted exergaming with Able-X and EL strategies: The findings.

2. Ten case studies: showcasing the stories of the successes experienced by the individual PWD who participated in this study (a book is planned to present these). 
3. Reinterpreting actions and behaviour of PWD through the lens of embodied selfhood and other sources of embodied memory.

4. The therapeutic influence of the researcher when engaging in psychosocial intervention research with PWD.

5. Using video data when conducting research with PWD.

6. Quality of life in PWD is often reported subjectively: Complementary objective data reduces the weaknesses in each data type. A parallel convergent model.

7. Facilitating computer-assisted exergaming therapy with people with dementia: Theoretical and methodological influences.

8. Gaining consent from vulnerable adults with dementia: Rights, risks, assent and dissent.

\subsection{Conclusion}

This study has demonstrated that improved hand-eye coordination and overall QOL were the results of computer-assisted exergaming with Able-X and EL strategies. Furthermore, irrespective of the stage of dementia, significant benefits can be achieved through solo or small group facilitated gaming sessions. It was also established that basic ADLs and IADLs capability and language ability is irrelevant to computer-assisted exergaming in partnership with a trained other. Through this research I have evidenced that PWD 'can do' even if they 'cannot think', and in so 'doing', are able, with assistance, to sentiently interact with novel technologies. Moreover, the findings of this study challenge the Cartesian view of mind-body dualism by establishing how people with AD and other dementias retain their embodied capability in the form of embodied selfhood (Kontos 2003, 2004), from which they are able to draw on pre-reflective bodily expression through movement, gestures and sociability, and socio-cultural dimension of the prereflective body. In addition, PWD are able to draw on two other pre-reflective resources: implicit or procedural bodily memory that allows the body to know without reasoning, and automatic bodily memory that allows the body to move without conscious thought or attention. In so doing, the body draws on the very essence of socio-cultural heritage that is primed and imbued within every fibre of the body through the human process of being present in and interacting with the world at any given time and in any given space. 


\subsection{Dedication}

The following poem embraces the key theoretical concepts of embodiment, errorless learning and unconditional and absolute value and respect. It also contains glimpses of the findings that emerged from this thesis. The poem is dedicated to all the participants and sympathetic others, who share the dementia journey and become wise through association.

\section{I am embodied}

I am changed in how I act, but I am me and that's a fact.

My memory's poor, my thinking too, but I'm still here and I can do.

I can re-learn and here's the key, one-step at a time for me.

I need help and that is true, but not with things that I can do.

I may repeat at rapid pace, but I am agent in this case.

I'm trying to orientate with grace to meet you in this time and space.

My bodily memory as you see, resides within the corporeal me.

My social self and culture too, and many things I learned to do

Are imbued within my very core, the primal me we can't ignore.

Tacitly and with intent, I will arise and reinvent.

I am of time; I am of space I would love to meet you in this place.

Poem by Jenny Drury-Ruddlesden (2016) 


\section{APPENDICES}

\section{Appendix 1: Invitation to residential unit}

TE WHARE WĀNANGA O TE ŪPOKO O TE IKA A MĀUI

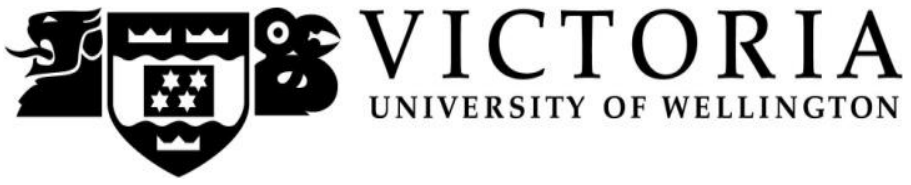

\section{INVITATION LETTER TO RESIDENTIAL UNITS TO PARTICIPATE}

Name and address of Residential/Care Home

Date:

\section{Cognitive Stimulation using computer gaming technology (Able-X) for people diagnosed with mild to moderate dementia}

Dear

I am a PhD candidate at the Graduate School of Nursing, Midwifery and Health at Victoria University of Wellington. My research is an observational study to evaluate cognitive stimulation using computer gaming technology (Able-X) for people diagnosed with mild to moderate dementia. My study is funded by the Callaghan Innovation Fund and is supported by Im-Able Ltd who developed the Able-X targeted at upper limb stroke recovery, and are providing me with the Abel-X to work with people with early to mid-stage dementia as a means of stimulating cognitive and physical activity in an effort to improve mental and physical functioning and to see if changes can be made to the Abel-X and the games, specifically for people with dementia.

The aim of this study is to establish if a programme of exer-game interactions, utilising computer gaming technology (Able-X) to deliver cognitive stimulation and enjoyment will improve cognitive functioning and wellbeing of people diagnosed with mild to moderate dementia. Computer technology (CT) is being increasingly used for the rehabilitation of people with dementia. In the pilot stage of the study, two to three participants will be recruited from one care home facility. The pilot will test the data collection processes as described below. Recommendations for changes will be made based on the usability of the technology for the people with dementia and appropriateness of the assessment scales. The interview schedule will be tested and further developed and video recording equipment tested. Also, the number of days per week/period over which the interaction with the computer technology 'games' will take place will be determined during the pilot stage. This stage should be completed by the end of January 2014.

The main study will establish if the interacting with (Able-X or a modified prototype) increases wellbeing for people with mild to moderate dementia and to continue to identify improvements of the Able-X for this specific group of people. 
This mixed methods study utilises Case Study methodology. A minimum of 20 participants from 3 to 6 care home settings will be recruited. The significance and benefits of the research include building on and contributing towards the body of knowledge that exists in the area of dementia rehabilitation and quality of life research and to contribute to the creation of intellectual property resulting in the possibility of a new or modified set of exer-game development in New Zealand for the commercial market specifically for people with dementia.

Participation in the study would involve allowing:

- the researcher access to appropriate accommodation to play the computer exer-games

- the use audio and video recording equipment;

- access to the health records of the relevant participants;

- administration of questionnaires to participants, family/whanau and staff (caregivers of participants)

- approach to participants (as identified by care home management)

- $\quad$ staff (caregivers of participants) being approached to participate in interview.

- family/whanau (of participants) being approached to participate in interview

I am an experienced researcher in dementia care and will have access to experienced supervisors whilst conducting this research.

Attached are the information letters that would be provided to participants in the study. I am also available to meet and discuss the study in more detail if this is required.

Yours truly

Jenny Drury-Ruddlesden

Jenny Drury-Ruddlesden

$\mathrm{PhD}$ Candidate/Research Associate

Graduate School of Nursing, Midwifery and Health

Victoria University of Wellington

PO Box 7625

Newtown

Wellington 6242 
Appendix 2: Access agreement

TE WHARE WĀNANGA O TE ŪPOKO O TE IKA A MĀUI

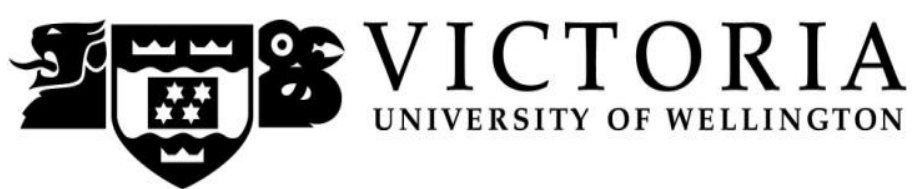

ACCESS AGREEMENT LETTER FROM RESIDENTIAL/CARE HOME

Cognitive stimulation using exer-games (Able-X) for people with

RESEARCHER

dementia

Jenny Drury-Ruddlesden

$\mathrm{PhD}$ Research Candidate/Research Associate

Graduate School of Nursing, Midwifery and Health

Victoria University of Wellington

PO Box 7625

Newtown

Wellington 6242

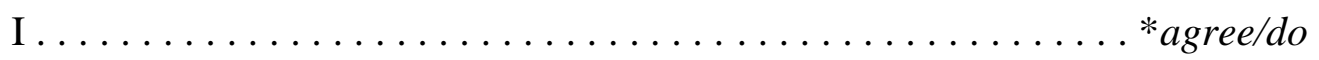
not agree to allow the residential unit to be involved in a research study on cognitive stimulation using exer-games (Able-X) for people with dementia and their family/whānau/caregivers. I agree to:

- allow the researcher access to appropriate accommodation to play the exer-games;

- use audio and video recording equipment;

- access the health records of the relevant participants;

- administer questionnaires to participants, family/whanau and staff (caregivers of participants)

- staff (caregivers of participants) being approached to participate in interview.

- to family/whanau (of participants) being approached to participate in interview

- [Delete as appropriate]

Organisation [Residential Care Home]:

Name:

Designation:

Telephone Number:

Email Address:

Date: 


\section{Appendix 3: Invitation to NOK}

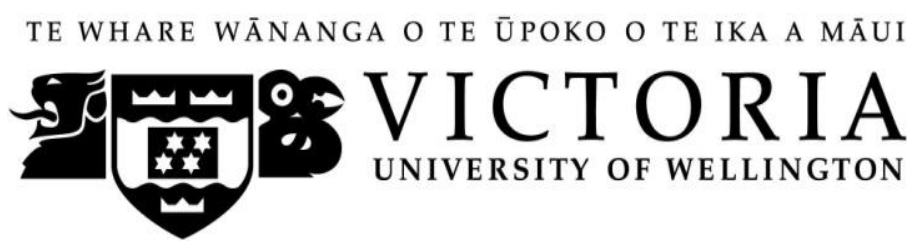

\section{INVITATION LETTER TO FAMILY/WHANAU/SIGNIFICANT OTHERS TO PARTICIPATE}

Name and address of NOK

Date:

\section{Cognitive Stimulation using computer gaming technology (Able-X) for people diagnosed with mild to moderate dementia}

Dear

This letter has been sent on my behalf by the management of the care home where your family member resides. Your family member has been identified as fitting the inclusion criteria for my research study.

I am a PhD candidate at the Graduate School of Nursing, Midwifery and Health at Victoria University of Wellington. My research is an observational study to evaluate cognitive stimulation using computer gaming technology (Able-X) for people diagnosed with mild to moderate dementia.

The aim of this study is to establish if a programme of exer-game interactions, utilising computer gaming technology (Able-X) to deliver cognitive stimulation and enjoyment will improve cognitive functioning and wellbeing of people diagnosed with mild to moderate dementia. Computer technology (CT) is being increasingly used for the rehabilitation of people with dementia. In the pilot stage of the study, two to three participants will be recruited from one care home facility. The pilot will test the data collection processes and usability of the computer game technology. Recommendations for changes will be made based on the usability of the technology for the people with dementia and appropriateness of the assessment scales. The interview schedule will be tested and further developed and video recording equipment tested. Also, the number of days per week/period over which the interaction with the computer technology 'games' will take place will be determined during the pilot stage. This stage should be completed by the end of January 2014.

The main study (commencing in April/May 2014) will establish if the interacting with (Able-X or a modified prototype) increases wellbeing for people with mild to moderate dementia and to continue to identify improvements of the Able-X for this specific group of people. 
This mixed methods study utilises Case Study methodology. A minimum of 20 participants from three to six care home settings will be recruited. The significance and benefits of the research include building on and contributing towards the body of knowledge that exists in the area of dementia rehabilitation and quality of life research and to contribute to the creation of intellectual property resulting in the possibility of a new or modified set of exer-game development in New Zealand for the commercial market specifically for people with dementia.

Participation in the study would involve allowing the researcher to:

- meet with family representative (s)

- use audio and video recording equipment;

- access to the health records of the relevant participants (person with dementia);

- administration of questionnaires to participants, family/whanau and staff (caregivers of participants)

- approach participants (as identified by care home management and agreed by nok/family)

- approach staff (caregivers of participants) to participate in interview.

- approach family/whanau (of participants) to participate in interview and completion of some brief questionnaires.

My study is funded by the Callaghan Innovation Fund and is supported by ImAble Ltd. Im-Able Ltd. are providing me with the Abel-X to work with people with early to mid-stage dementia as a means of stimulating cognitive and physical activity in an effort to improve mental and physical functioning and to see if changes can be made to the Abel-X and the games, specifically for people with dementia.

I am an experienced researcher in dementia care and will have access to experienced supervisors whilst conducting this research.

Attached are the information letters that would be provided to all participants in the study.

Appendix $\mathrm{C}$ is the detailed information sheet and contains consent forms (for all participants, including person with dementia, family members and staff/carers).

Appendix I is a simplified information sheet and consent for the person with dementia

Appendix $\mathrm{E}$ is a proxy agreement to approach person with dementia (Must be signed by the next of kin or Enduring Power of Attorney).

If you hare happy to be part of my research, please sign and return Appendix $\mathrm{C}$ and Appendix $\mathrm{E}$ in the envelope provided. I will copy the signed documents and return a copy to you. 
I will be commencing the pilot stage in early January and I am looking for up to three participants for the pilot study.

Yours truly

Jenny Drury-Ruddlesden

$\mathrm{PhD}$ Candidate/Research Associate

Graduate School of Nursing, Midwifery and Health

Victoria University of Wellington

PO Box 7625

Newtown

Wellington 6242 
Appendix 4: Study information and consent

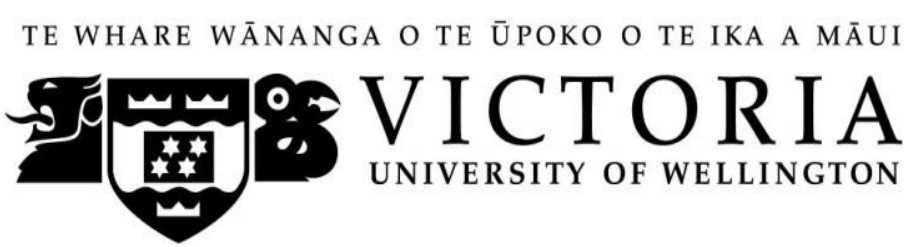

\section{STUDY PARTICIPANT AND SURROGATE PARTICIPANT INFORMATION SHEET}

Study Title: Cognitive stimulation using exer-games (Able-X) for people with dementia

Locality: General Scope Care Facilities

Lead Jenny Drury-Ruddlesden investigator:
Ethics committee ref: 13/CEN/135

You are invited to take part in a study on a programme of exer-game interventions, utilising computer gaming technology (Able-X) to deliver cognitive stimulation and enjoyment in an effort to improve cognitive functioning and wellbeing of people with mild to moderate dementia (study participant). You may be either the study participant or the surrogate participant $^{41}$ (a family/whānau member/significant other/staff member/care giver). Whether or not you take part is your choice. If you do not want to take part, you do not have to give a reason, and it will not affect you in anyway. If you do want to take part now, but change your mind later, you can pull out of the study at any time.

This Participant Information Sheet will help you decide if you would like to take part. It sets out why we are doing the study, what your participation would involve, what the benefits and risks are, and what would happen after the study ends. I will go through this information with you and answer any questions you may have. You do not have to decide today whether you will participate in this study. Before you decide, you may want to talk about the study with other people, such as family, whānau, friends, or healthcare providers. Feel free to do this.

If you agree to take part in this study, you will be asked to sign the Consent Form on the last page of this document. You will be given a copy of both the Participant Information Sheet and the Consent Form to keep.

This document is [6] pages long, including the Consent Form. Please make sure you have read and understood all the pages.

\footnotetext{
${ }^{41}$ A surrogate, sometimes called a 'proxy' is a family/whānau member, caregiver or other trusted individual who knows the study participant well and can assist with the consenting and assessment processes of this research.
} 


\section{WHAT IS THE PURPOSE OF THE STUDY?}

The purpose is:

To explore the use of the Able-X or a modified prototype to provide cognitive stimulation and for people with mild to moderate dementia.

The objectives are to:

To assess whether people who are resident in care homes and have mild to moderate stage dementia achieve 1. increased quality of life and pleasurelenjoyment; 2. increased cognitive functioning and 3. increased physical function as a result of using Able-X.

To test Able-X system to establish efficacy, usability and functionality when used by people with dementia.

\section{WHAT WILL MY PARTICIPATION IN THE STUDY INVOLVE?}

You have been invited because you are a staff member / caregiver / family/whanau / significant other (surrogate) of a study participant residing in a care home who has been identified by care home management as fitting the criteria for this study (see table 1below).

\section{Inclusion and exclusion criteria}

\begin{tabular}{|c|c|}
\hline Inclusion Criteria & Exclusion Criteria \\
\hline $\begin{array}{l}\text { - Age } 65 \text { or over. } \\
\text { Diagnosis of dementia or } \\
\text { showing signs of memory } \\
\text { impairment as determined by } \\
\text { care home management and/or } \\
\text { family members if appropriate } \\
\text { - The level of dementia is mild to } \\
\text { moderate } \\
\text { - } \text { Has resided in a care facility for } \\
\text { at least one year } \\
\text { - } \text { Speaks English as first language } \\
\text { - Ability to follow simple } \\
\text { instructions in English. } \\
\text { - Ability to physically hold the } \\
\text { gaming device and engage with } \\
\text { computer technology as judged } \\
\text { by the researcher and/or care } \\
\text { home staff /family/whānau } \\
\text { /significant other. }\end{array}$ & 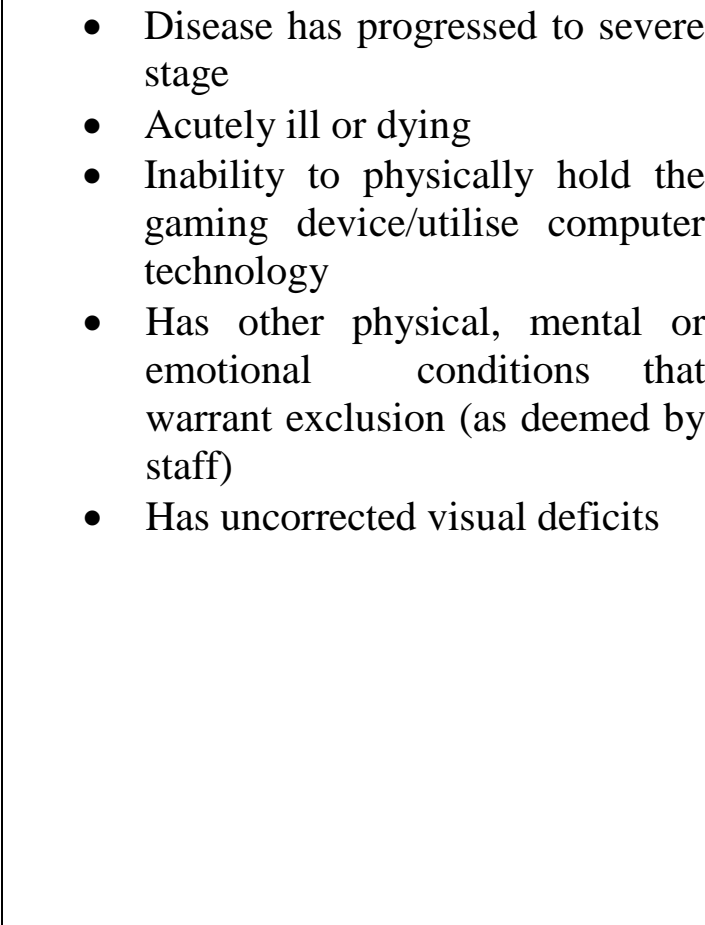 \\
\hline
\end{tabular}

Your assistance in the consenting process is required for this study. In addition, your views and observations in respect to any changes that you notice during the study would be very useful in helping me to understand the effects of playing with the Able-X by people with dementia in a care home environment. I also need your help completing some of the assessments. 
Once you have agreed to take part in the project, you will be asked to participate in the consenting process, helping me complete some assessment questionnaires and finally, to take part in an audio-recorded interview. I will ask you what changes you have noticed (if any) over the last few weeks - whilst I have been helping the study participant to use the Able-X to play the games (you are welcome to come to play sessions). It is anticipated that the interview will last about 45 minutes to an hour. The interview will be conducted at a negotiated date and time in a quiet room at the Care home. To be available as required to complete up to 2 assessments about the study participant (this is called being a surrogate for that person). Completion of the assessment scales will take between 10 to 15 minutes of your time. These will be completed at midway and end points of the four-six week study.

\section{WHAT ARE THE POSSIBLE BENEFITS AND RISKS OF THIS STUDY?}

There are no anticipated risks or discomfort associated with participating in this study. As I am assessing for cognitive functioning, depression and anxiety, I have a duty to share any unexpected severe results with the participant's primary care giver and GP, in the best interest of the study participant. You will be notified if this action is taken.

All views expressed will be respected and treated in confidence.

Benefits will be that the research will provide a greater understanding of the use of computer game playing by people with dementia and whether playing these games increases their wellbeing.

\section{WHO PAYS FOR THE STUDY?}

There are no payments related to this study. I will bring the computer and game playing equipment to each session.

\section{WHAT IF SOMETHING GOES WRONG?}

There is no anticipated risk in taking part in this research.

\section{WHAT ARE MY RIGHTS?}

Participation is voluntary. You are free to decline to participate, or to withdraw from the research at any practicable time without experiencing any disadvantage.

You have the right to access information about data collected as part of the study.

If any participants become distressed during the game playing, the exercise will be discontinued.

All data, including persons and organisation details gathered in the course of this study will be de-identified. 


\section{WHAT HAPPENS AFTER THE STUDY OR IF I CHANGE MY MIND?}

You and/or the study participant are free to withdraw at any time you choose. I cannot promise the study will help you directly but I hope the information I get from this study will help improve the wellbeing of older people with dementia.

All data will be stored securely, paper documents and video imagery will be locked in filing cabinets in an office at the Graduate School of Nursing Midwifery and Health (Victoria University of Wellington). Electronic data will be stored on a password protected laptop computer. The data will be reviewed by the researcher, and academic supervisors. The transcriber will be asked to sign a confidentiality agreement. The video imagery will be viewed by representatives from industry as part of developing the software and hardware for people with dementia. Viewing will be in a controlled and private environment, in my presence. No copies of the video imagery will be made other than the original. Data will be stored for 10 years and then destroyed.

The results will be written up as a report at the end of the study. You will be offered a summary report of the findings at the completion of the study. If you change your mind during the study, we will negotiate what happens to the data already collected.

\section{WHO DO I CONTACT FOR MORE INFORMATION OR IF I HAVE CONCERNS?}

If you have any questions, concerns or complaints about the study at any stage, you can contact:

Jenny Drury-Ruddlesden.

PhD Candidate /Research Associate

Graduate School of Nursing, Midwifery and Health

Victoria University of Wellington

Wellington

You can also contact the health and disability ethics committee (HDEC) that approved this study on:

Phone: $\quad 08004$ ETHICS

Email: $\quad$ hdecs@moh.govt.nz

Ref: $\quad$ 13/CEN/135 
Appendix 5: Simplified information sheet for PWD

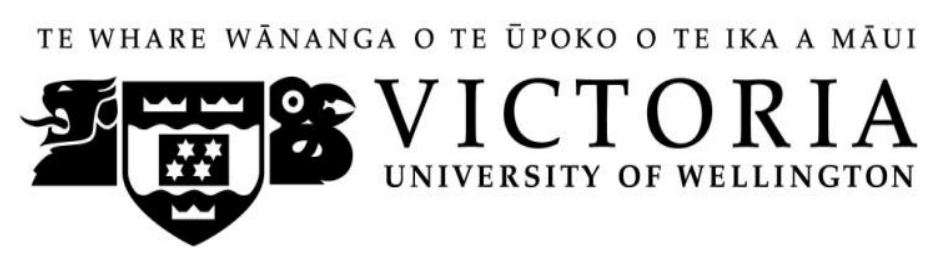

INFORMATION SHEET - STUDY PARTICIPANT

Cognitive stimulation using exer-games (Able-X) for people with dementia

This study aims to find out if playing the computer game helps people with memory loss:

- You do not have to take part

- You can withdraw at any time

- Before we start I will ask you some questions

- I will then show you how to play the computer games

- We will play for up to 40 minutes each time

- We will play 3 times a week

- We will do this for 4 weeks

- I will video you playing the games

- I will ask some of your caregivers and family members questions too

- Abnormal assessment results will be sent to your GP and primary caregiver

- All other information will be confidential

- All data will be kept securely for 10 years and then destroyed.

Contact details:

Jenny Drury-Ruddlesden (Principle Investigator).

$\mathrm{PhD}$ Candidate/Research Associate

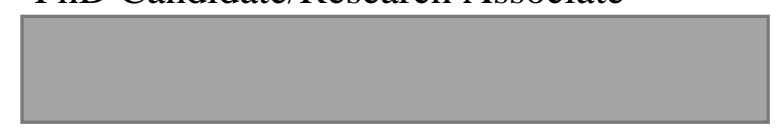

You can also contact the health and disability ethics committee (HDEC) that approved this study on:

$\begin{array}{ll}\text { Phone: } & 0800423638 \\ \text { Email: } & \text { hdecs@ moh.govt.nz } \\ \text { Ref: } & \text { 13/CEN/135 }\end{array}$




\section{Declaration by study participant}

I hereby consent to take part in this study

Study Participant's Name:

Signature:

Date:

e-mail /address for summary report and

photocopy of consent form:

\section{Declaration by member of research team:}

I have given an oral explanation of the research project to the study participant and have answered the participant's questions.

I believe that the participant understands the study and has consented/assented to participate in this study.

Researcher's name: Jenny Drury-Ruddlesden

Signature:

Date: 
Appendix 6: Proxy agreement

TE WHARE WĀNANGA O TE ŪPOKO O TE IKA A MĀUI

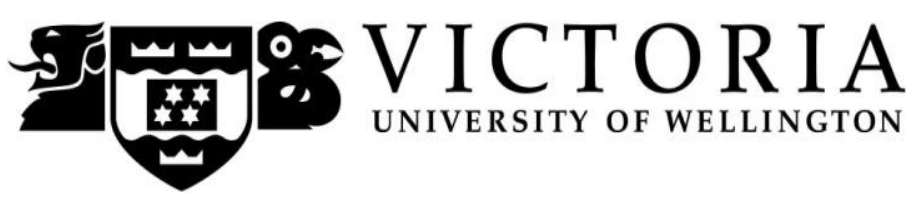

\section{PROXY AGREEMENT TO APPROACH PERSON WITH DEMENTIA LETTER}

Cognitive stimulation using exer-games (Able-X) for people with

RESEARCHER dementia

Jenny Drury-Ruddlesden

$\mathrm{PhD}$ Research candidate/Research Associate

Graduate School of Nursing, Midwifery and Health

Victoria University of Wellington

PO Box 7625

Newtown

Wellington 6242

I . . .............................. * agree/do not agree to allow the researcher, identified above to approach: Name: to discuss taking part in this research study 'cognitive stimulation using exer-games (Able-X) for people with dementia'.

I understand that:

- the researcher will assist and supervise the playing of the exer-games

- use audio and video recording equipment

- access the above named health records

- administer questionnaires to assess mental health and wellbeing to participants, family/whanau and staff (caregivers of participants)

- $\quad$ staff (caregivers of participants) may be approached to participate in interview.

- to family/whanau (of participants) being approached to participate in completion of questionnaires

- [Delete as appropriate]

Name:

Relationship to the above named:

Telephone Number:

Email Address:

Date: 


\section{Appendix 7: Summary of Able-X}

Able-X $\mid$ Im-Able

Im-Able

|Home About | Research | Customer Support | Contact | Products

A.ble:X Key Grip Orhos:s

\section{Able-X}

Accelerated stroke recovery

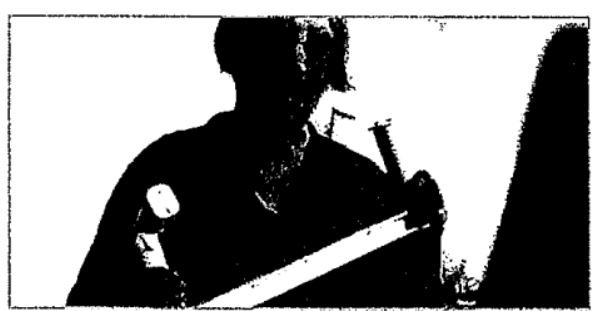

The Able-X accelerates arm and memory recovery after stroke. Able-X exercise "games" were designed by health professionals to stimulate and retrain the brain

1. The system is designed to prompt greater left brain - right brain stimulation

2. All exercises are shown as games to increase enjoyment for user

3 The system is designed to increase the number of repititions per exercise session

Who is the Able-X System Designed For?

Able-X is suilable for children and adults with a wide range of brain and muscle coortination impairments e.a stroke. and cerebral palsy.

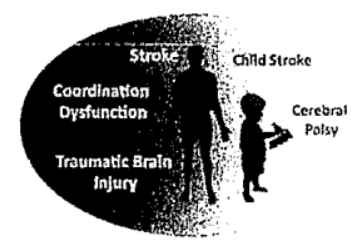

How Does the Able-X System Benefil Users?

$$
\begin{aligned}
& \text { The product was formally :ested on chronic stroke } \\
& \text { patients ( } 15 \text { months to } 7 \text { years post stroke event) } \\
& \text { at Olago School of thedicine. New Zealand } \\
& \text { Regular users of the system gained benefits, } \\
& \text { some more than others. These include' } \\
& \text { ability to use both trands on steering wheet: } \\
& \text { abifity to tie shoelaces: } \\
& \text { eat using both hands with knife and lork; } \\
& \text { better control in shower: } \\
& \text { better body balance: } \\
& \text { more control of wheelchair } \\
& \text { improventent to memory and concentration }
\end{aligned}
$$

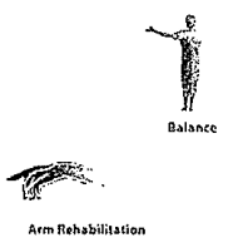

Arem Rehabilitation

Users were assessed befote the trial and after the trial of 9 sessions ( 1 hour each).
Page 1 of 3

APPENDIX F

$S E \approx P Z_{-} H$

Call us on 07742099343

\section{Navigation}

wore information

Insiructional Video

Demonstration

Testimonials

Clinical trials

FACs

Troubleshooting

Shopping cart

Oltems

Total: 20.00

Key Factors in Brain Recovery

Info for Health Professionals

Download PDF here

How Able-X works

Info for users \& caregivers

Downioad PDF here 
User feedback can be viewed at htlp://Www.im-able.com/nz/node/18

How Does the Able-X System Work?

The success of the Able-X System is attribuled to three key factors:

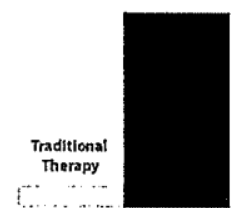

High Levels of Repetitions for Exercises

The Able- $X$ can achieve 800 repelitions in an hour whereas the published results for traditional theropy is estimated to be 32 repelitions per hour.

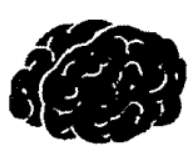

Exercises designed to prompt left brain - righ brain stimulation.

Tatgets appear from and move in different directions on the screen requining movement from both arms, and concentration both sides of the brain.

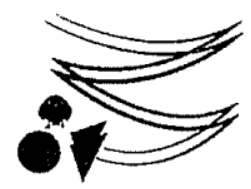

Exercises are embodied in game format to increase enjoyment factor.

Holding the handleber, your arms conitrol the position of the cursor on the screen, by moving as you play the gamos.

\section{Awards}

Im-Able has been recognized by the following organisations for our work:

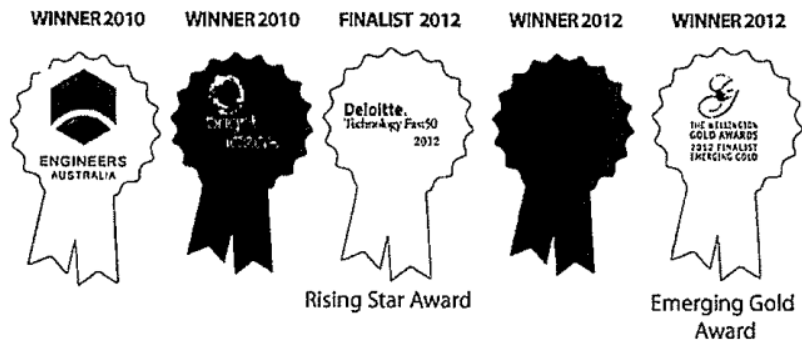

Able-X: First Prize - Blomedical Engineers Australia. 2010

Able-X: First Prize - Bright Ideas Competition, 2010

Im-Able: Winner - Emerging Gold, Wellinglon, 2012

Im-Abte: Winner. ANZ Flying Start Award, 2012

Im-Able: Finalis! - 2012 Deloilte Fast 50 Rising Star Award - finalist

Clinical results can be vigwed at hutp://mmw.im-able.com/nz/published-papers

Require more information? contact info@im-able.com

Able-X has FDA 510k clearance (USA) as a Closs 1 Medical Device and CE cerlification (Europe) as a Class Medical Device.

Downioad Brochure here

Add to cart

Information for... 
Appendix 8: Health record review

TE WHARE WĀNANGA O TE ŪPOKO O TE IKA A MĀUI

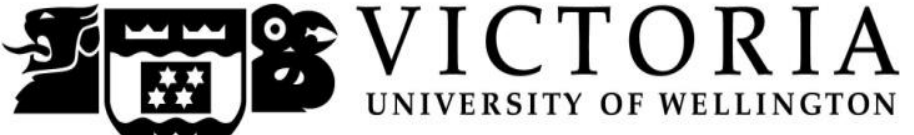

\begin{tabular}{|c|c|c|}
\hline \multicolumn{2}{|c|}{$\begin{array}{l}\text { DATA COLLECTION FORM AND HEALTH RECORD REVIEW } \\
\text { All Questions Pre-Intervention/Q19 onwards post-intervention) }\end{array}$} & \multirow{2}{*}{$\frac{\text { Subject/Location Code }}{\text { Comments }}$} \\
\hline Demographics/Admission details & Response & \\
\hline 1. Gender: & & \\
\hline 2. Date of birth: & & \\
\hline 3. Ethnicity: & & \\
\hline 4. $\quad$ Spoken First Language: & & \\
\hline 5. Spoken second language & & \\
\hline 6. $\quad$ Religious/Spiritual affiliations & & \\
\hline 7. Cultural affiliations documented & & \\
\hline 8. Smoking history: & & \\
\hline 9. Date of admission: & & \\
\hline 10. Communication skill & & \\
\hline 11. Glasses needed? & & \\
\hline 12. Hearing aids need? & & \\
\hline 13. Mobility? & & \\
\hline 14. Any factors that may cause distress? & & \\
\hline 15. Actions that will calm if distressed & & \\
\hline
\end{tabular}




\begin{tabular}{|c|c|c|}
\hline \multicolumn{2}{|l|}{ Section 2: Assessment/Continuing Care Details } & \multirow[t]{2}{*}{ Free text } \\
\hline 16. Co-Morbidities recorded: & $\begin{array}{ll}\text { 1. } & \text { Arthritis } \square \\
\text { 2. } & \text { Cancer } \square \\
\text { 3. } & \text { Cardiovascular Disease } \square \\
\text { 4. } & \text { Cerebrovascular Disease } \square \\
\text { 5. } & \text { COPD } \square \\
\text { 6. } & \text { Diabetes } \square \\
\text { 7. } & \text { Other- Free text : }\end{array}$ & \\
\hline $\begin{array}{l}\text { 17. Reference to dementia/cognitive } \\
\text { impairment/memory loss in notes: }\end{array}$ & $\begin{array}{l}\text { 1. Yes } \square \text {. NoD } \\
\text { If yes record all terms used (free text): }\end{array}$ & \\
\hline \multirow[t]{4}{*}{ 18. Recorded diagnosis of dementia: } & $\begin{array}{l}1 . \quad \text { Yes } \square \\
\text { If yes record: }\end{array}$ & \multirow[t]{3}{*}{ Comments } \\
\hline & Date: & \\
\hline & $\begin{array}{l}\text { Recorder } \\
\text { 1. GP } \\
\text { 2. Consultant } \\
\text { 3. Other (free text) }\end{array}$ & \\
\hline & Pre-Intervention & Post Intervention \\
\hline \multicolumn{3}{|l|}{ 19. Cognitive status: } \\
\hline $\begin{array}{l}\text { 19a.Depression: Assessment scales used e.g. geriatric } \\
\text { depression scale (Identify scale used): }\end{array}$ & 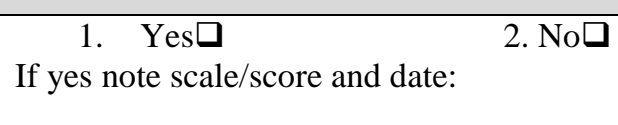 & 1. Yes $\square$ \\
\hline
\end{tabular}




\begin{tabular}{|c|c|c|}
\hline 19b. Depression: Clinical Judgment: & $\begin{array}{l}1 . \text { Yes } \\
\text { If yes note comments/terminology }\end{array}$ & 1. Yes $\square$ \\
\hline 19c. Dementia: Assessment scales used e.g. & $\begin{array}{cc}1 . \quad \text { Yes } \square & 2 . \mathrm{No} \square \\
\text { If yes note scale/score and date: }\end{array}$ & $1 . Y e s \square$ \\
\hline 19d. Dementia: Clinical Judgment: & $\begin{array}{l}1 . \text { Yes } \square \\
\text { If yes note comments/terminology }\end{array}$ & 1. Yes $\square$ \\
\hline 19e. Anxiety: Assessment scales used e.g. RAID & $\begin{array}{cc}1 . \quad \text { Yes } & 2 . \mathrm{No} \\
\text { If yes note scale/score and date: }\end{array}$ & 2. No $\square$ \\
\hline 19f. Anxiety: Clinical Judgment: & $\begin{array}{c}2 . \quad \text { Yes } \\
\text { If yes note comments/terminology }\end{array}$ & 1. Yes $\square$ \\
\hline 19g. Agitated behaviour: & 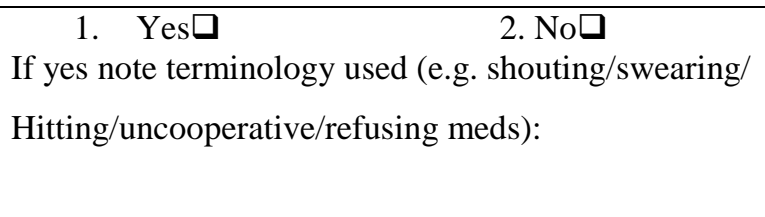 & 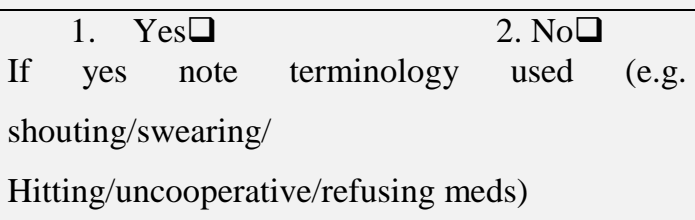 \\
\hline
\end{tabular}




\begin{tabular}{|l|l|}
\hline Section 3: Medications: & \\
\hline 20. Medications on Admission: List (Pre-Intervention): & \\
\hline 21. Notes: Pre (free text) PTO & 20b. Medications on Admission: List (Post-Intervention): \\
& \\
\hline
\end{tabular}

Jenny Drury-Ruddlesden

PhD Candidate/Research Associate

Graduate School of Nursing, Midwifery and Health

Victoria University of Wellington

PO Box 7625

Newtown

Wellington 6242

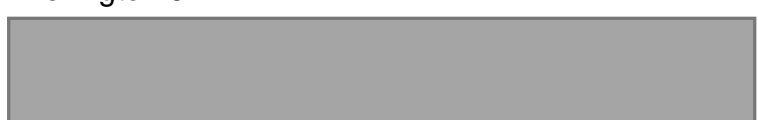


Appendix 9: Interview Schedule

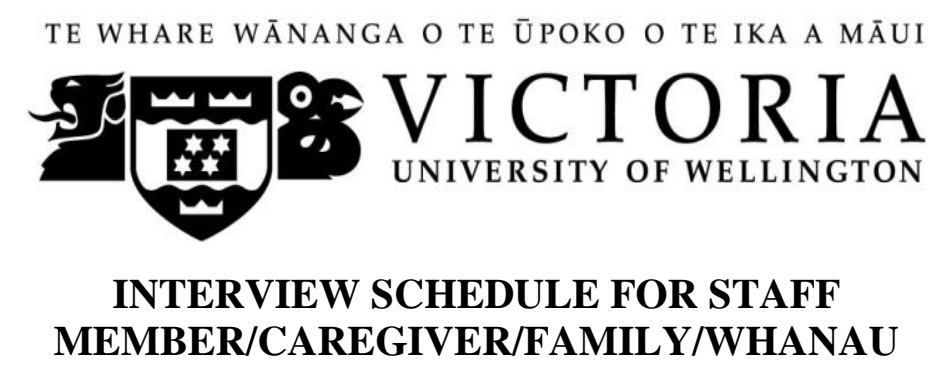

\section{Cognitive stimulation using exer-games (Able-X) for people with dementia}

Principle Investigator: Jenny Drury-Ruddlesden, PhD candidate, Graduate School of Nursing, Midwifery and Health, Victoria University of Wellington (VUW)

Participant Name:

Relationship: :Date:

\section{PRE-INTERVIEW:}

1. Fill with gaps in Data collection/Health Record Review

2. Turn recorder on (with permission)

3. State name, date and time on to the recorder

4. State study participant (SP) and location code on to the recorder

5. Ask all present to say who they are and their association with study participant

6. Reassure that all names, if used will be coded and edited out of transcripts

7. Would you (or your family members) like to attend some of the gaming sessions?

8. If not - would you be prepared to attend at least one?

\section{BASELINE INTERVIEW:}

a. Can you describe $\mathrm{x}$ in one sentence that would give me an overview of her very essence?

b. RN only - How long have you been primary carer for $\mathrm{x}$ ?

\section{Happiness:}

1. How do you judge if (SP) is happy or not?
a. Give examples.
b. What makes (SP) happy?
c. What makes (SP) unhappy?
d. In what ways does (SP) express happiness?

\section{Enjoyment and Pleasure}

2. What pleasurable activities does (SP) engage in?
a. In what ways does (SP) express enjoyment and pleasure?
b. Are there any particular activities or games that (SP) enjoys? 


\section{Quality of life:}

3. Does (SP) have a good quality of life?
a. Give examples.
b. How could (SP) QOL be improved?
c. Are you aware of any creating coping strategies that (SP) or family/carers use (give example of cup of tea or wee bucket) that have been implemented both before admission to the unit and since admission?

\section{Using a computer}

4. Has (SP) ever played a computer game before?

5. Has (SP) ever used a computer?

\section{Other:}

6. Education - How many years of schooling did (SP) have (finish high school or university?)

7. What is (SP) marital status/partnership/closest relationship?

8. Is there anything else you would like to add that I haven't asked about and you think I should know?

\section{Turn recorder off}

9. Complete other measures as appropriate

\section{Midpoint and End point interview}

Participant Name:

Relationship:

:Date:

\section{Playing on Abel-X}

10. Have you noticed any changes since we started using the Able-X and playing the computer games?
a. If yes, give examples of what you noticed.
b. How were they demonstrating what they did?
c. Do they seem to get pleasure and enjoyment out of playing?
d. If so, how do you know?
e. How would I recognize it?
f. Does (SP) appear happier since we have been playing the games?
g. If so, how do you know?
h. How would I recognize it?
i. How could the game be improved for (SP) and others with dementia?
i. Interface
ii. Actual games
iii. Other:

11. Is there anything else you would like to add?

12. Complete other measures as appropriate

13. Thank you so much for taking part in my research. 


\section{End point interview}

Participant Name:

Relationship:

:Date:

\section{Playing on Abel-X}

14. Have you noticed any changes since we started using the Able- $X$ and playing the computer games?

a. If yes, give examples of what you noticed.

b. How were they demonstrating what they did?

c. Do they seem to get pleasure and enjoyment out of playing?

d. If so, how do you know?

e. How would I recognize it?

f. Does (SP) appear happier since we have been playing the games?

g. If so, how do you know?

h. How would I recognize it?

i. How could the game be improved for (SP) and others with dementia?

i. Interface

ii. Actual games

iii. Other:

15. Is there anything else you would like to add?

16. Complete other measures as appropriate.

17. Thank you so much for taking part in my research.

Jenny Drury-Ruddlesden

$\mathrm{PhD}$ Candidate/Research Associate

Graduate School of Nursing, Midwifery and Health

Victoria University of Wellington

PO Box 7625

Newtown 
Appendix 10: Confidentiality agreement

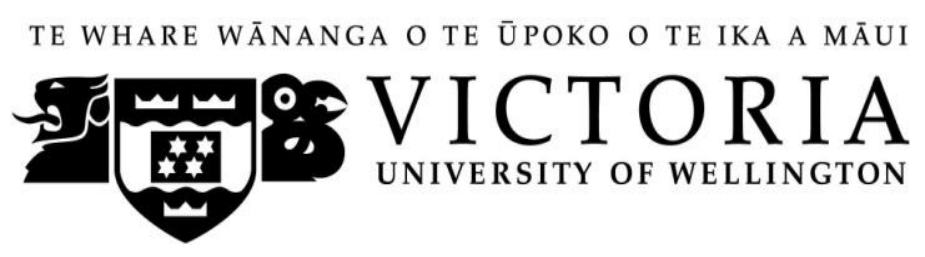

\title{
Confidentiality Agreement for
}

Research study titled: Cognitive stimulation using exer-games (Able-X)

for people with dementia

Researcher: Jenny Drury-Ruddlesden

\begin{abstract}
ABLE-X ADVISOR (please delete role below)
I have read the information sheet outlining this study. I have discussed with the researcher the nature of the research and have had any questions that I have had answered to my satisfaction. My role as the research Advisor (Able-X) has been outlined to me by the researcher.
\end{abstract}

At all times the research information (video recordings) will be inaccessible to other persons and will be viewed with the researcher. The researcher has assured me that she will debrief me following transcribing to address any issues that observing the videos brings up for me.

Most importantly, I understand and agree to keep the information I see and hear in the course of viewing is confidential to the researcher and myself.

\section{TRANSCRIBER (Please delete role above)}

I have read the information sheet outlining this study. I have discussed with the researcher the nature of the research and have had any questions that I have had answered to my satisfaction. My role as the research Transcriber has been outlined to me by the researcher.

At all times the research information (tapes, video recordings and transcripts) will be inaccessible to other persons. The researcher has assured me that she will debrief me following transcribing to address any issues that transcribing bring up for me.

Most importantly, I understand and agree to keep the information I hear and type in the course of transcribing confidential to the researcher and myself.

Full Name:

Signature:

Date:

Researcher's Signature:

Date: 


\section{Appendix 11: Ethics approval}

Health
and
Disability
Ethics
Committees.

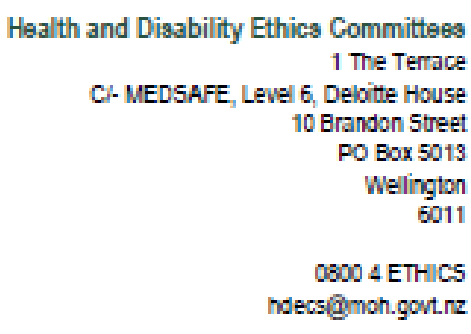

Health and Disability Ethics Committees 1 The Terrace Cl-MEDSAFE, Level 6 , Deloitte House 10 Brandon Street PO Box 5013 Wellington 6011

06004 ETHIICS ndecs@mon.govt.nz

11 November 2013

Mrs Jeanette Drury-Ruddlesden

PO Box 7625

Newtown 6242

Dear Mrs Drury-Ruddlesden

\begin{tabular}{|c|c|c|}
\hline \multirow[t]{2}{*}{ Re: } & Ethics ref: & 13/CEN/135 \\
\hline & Study title: & $\begin{array}{l}\text { Cognitive stimulation using computer gaming technology (Able-X) for } \\
\text { people diagnosed with mild to moderate dememtia. }\end{array}$ \\
\hline
\end{tabular}

I am pleased to advise that this application has been approved by the Central Health and Disability Ethics Committee. This decision was made through the HDEC-Full Review pathway.

\section{Conditions of HDEC approval}

HDEC approval for this study is subject to the following conditions being met prior to the commencement of the study in New Zealand. It is your responsibility, and that of the study's sponsor, to ensure that these conditions are met. No further review by the Central Health and Disability Ethics Committee is required.

Standard conditions:

1. Before the study commences at any locality in New Zealand, all relevant regulatory approvals must be obtained.

2. Before the study commences at a given locality in New Zealand, it must be authorised by that locality in Online Forms. Locality authorisation confirms that the locality is suitable for the safe and effective conduct of the study, and that local research governance issues have been addressed.

Non-standard conditions: please remove "there are no risks" The elderly are unstable and could slip.

Please submit Non-standard conditions to HDECS@moh.govtnz for completeness. Do not submit Non-standard conditions as a Post Approval form.

\section{After HDEC review}

Please refer to the Standard Operating Procedures for Health and Disability Ethics Committees (available on www.ethics.health.govt.nz) for HDEC requirements relating to amendments and other post-approval processes. 


\section{REFERENCES}

Aguirre, E., Spector, A., Hoe, J., Russell, I.T., Knapp, M., Woods, R.T., \& Orrell, M. (2010). Maintenance cognitive stimulation therapy (CST) for dementia: A singleblind, multi-centre, randomized controlled trial of Maintenance CST vs. CST for dementia. Trials, 11(1), 46-46. doi:10.1186/1745-6215-11-46

Alexopoulos, G.S (2002). The Cornell Scale for Depression in Dementia: Administration and Scoring Guidelines. NY, USA: Cornell Institute of Geriatric Psychiatry.

Alexopoulos, G.S., Abrams, R.C., Young, R.C., \& Shamoian, C.A. (1988). Cornell Scale for Depression in Dementia. Biological Psychiatry, 23(3), 271-284. Retrieved from doi:http://dx.doi.org/10.1016/0006-3223(88)90038-8

Alley, S., Jackson, S.F., \& Shakya, Y.B. (2015). Reflexivity: A methodological tool in the knowledge translation process? Health Promotion Practice, 16(3), 426-431. doi: $10.1177 / 1524839914568344$

Alzheimer's Association. (2011). Protection of participants in research. Retrieved from http://www.alz.org/documents_custom/statements/protection_of_ participants_in_research.pdf

Alzheimer's Association. (2013a). Alzheimer's disease facts and figures Alzheimer's \& dementia (Vol. 9). Washington, DC: Alzheimer's Association, Public Policy Office, Retrieved from http://www.alz.org/downloads/facts_figures_2013.pdf

Alzheimer's Association. (2013b). Types of dementia. Retrieved from http://www.alz.org/dementia/types-of-dementia.asp

Alzheimer's Association. (2013c). What is dementia? Retrieved from http://www.alz.org/what-is-dementia.asp

Alzheimer's Association (2015a). Medications for memory loss. Retrieved from http://www.alz.org/alzheimers_disease_standard_prescriptions.asp

Alzheimer's Association (2015b). Treatments for behaviour. Retrieved from http://www.alz.org/alzheimers_disease_treatments_for_behavior.asp

Alzheimers New Zealand. (2010). National Dementia Strategy 2010-2015. Wellington, New Zealand: Author. Retrieved from http://www.alzheimers.org.nz/files/reports/National_Dementia_Strategy_20102015.pdf

Alzheimers New Zealand. (2012). Economic impact of dementia in New Zealand 2012: Executive summary. Wellington, New Zealand: Author. Retrieved from http://www.alzheimers.org.nz/getmedia/2fa9d0e6-16dd-483a-97304216f9aaccb5/DEIR_2012_Executive_Summary.pdf.aspx

Alzheimers New Zealand Incorporated. (2008). Dementia economic impact report 2008. Wellington, New Zealand: Alzheimers New Zealand National Office. Retrieved from http://www.alzheimers.org.nz/files/reports/Dementia Economic_Impact_Report2008.pdf

Alzheimer's Research Trust. (2010). Dementia 2010: The economic burden of dementia and associated research funding in the United Kingdom. Oxford, UK: Health Economics Research Centre, University of Oxford for the Alzheimer's Research Trust. Retrieved from http://www.dementia2010.org/reports/ Dementia2010Full.pdf

Alzheimer's Society UK. (2013). The later stages of dementia: Factsheet 417LP. Retrieved from https://www.alzheimers.org.uk/site/scripts/download _info.php?downloadID $=1100$

American College of Sports Medicine (2013). Exergaming. Retrieved from www.acsm.org/docs/brochures/exergaming.pdf 
Amerson, R. (2011). Making a case for the case study method. Journal of Nursing Education, 50(8), 427-428. DOI: 10.3928/01484834-20110719-01

Aminzadeh, F., Byszewski, A., Molnar, F.J., \& Eisner, M. (2007). Emotional impact of dementia diagnosis: Exploring persons with dementia and caregivers' perspectives. Aging \& Mental Health, 11(3), 281-290.

Anthony, S., \& Jack, S. (2009). Qualitative case study methodology in nursing research: An integrative review. Journal of Advanced Nursing, 65(6), 1171-1181. doi: 10.1111/j.1365-2648.2009.04998.x

Astell, A.J., Ellis, M., Alm, N., Dye, R., Campbell, J., \& Gowans, G. (2004). Facilitating communication in dementia with multimedia technology. [Article]. Brain \& Language, 91(1), 80-81. doi: 10.1016/j.bandl.2004.06.043

Astell, A.J., Ellis, M., Alm, N., Dye, R., Gowans, G., \& Campbell, J. (2009). Developing technology to meet psychosocial needs. Journal of Dementia Care, 17(1), 36-38.

Astell, A.J., Ellis, M.P., Bernardi, L., Alm, N., Dye, R., Gowans, G., \& Campbell, J. (2010). Using a touch screen computer to support relationships between people with dementia and caregivers. Interacting with Computers, 22(4), 267-275. doi: http://dx.doi.org/10.1016/j.intcom.2010.03.003

Auer, S., \& Reisberg, B. (1997). The GDS/FAST staging system. International Psychogeriatrics, 9 (Supplement S1), 167-171. doi:10.1017/ S1041610297004869

Baddeley, A., \& Wilson, B.A. (1994). When implicit learning fails: Amnesia and the problem of error elimination. Neuropsychologia, 32(1), 53-68. doi: http://dx.doi.org/10.1016/0028-3932(94)90068-X

Ballard, C., Khan, Z., Clack, H., \& Corbett, A. (2011). Nonpharmacological treatment of Alzheimer disease. The Canadian Journal of Psychiatry, 56(10), 589-595. Retrieved from http://search.proquest.com/ docview/904985407? accountid=14782

Bamford, C., Eccles, M., Steen, N., \& Robinson, L. (2007). Can primary care record review facilitate earlier diagnosis of dementia? Family Practice, 24(2), 108-116. doi: 10.1093/fampra/cml068

Bamford, C., Lamont, S., Eccles, M., Robinson, L., May, C., \& Bond, J. (2004). Disclosing a diagnosis of dementia: A systematic review. International Journal of Geriatric Psychiatry, 19(2), 151-169. doi: 10.1002/gps.1050

Banerjee, S., Smith, S., Lamping, D., Harwood, R., Foley, B., Smith, P., . . . Mann, A. (2006). Quality of life in dementia: More than just cognition. An analysis of associations with quality of life in dementia. Journal of Neurology, Neurosurgery \& Psychiatry, 77(2), 146-148. doi: 10.1136/jnnp.2005.072983. (AN: 20201117)

Banerjee, S., \& Wittenberg, R. (2009). Clinical and cost effectiveness of services for early diagnosis and intervention in dementia. International Journal of Geriatric Psychiatry, 24(7), 748-754. doi: 10.1002/gps.2191

Baxter, P., \& Jack, S. (2008). Qualitative case study methodology: Study design and implementation for novice researchers. The Qualitative Report, 13(4), 544-559.

Beard, R.L. (2004). In their voices: Identity preservation and experiences of Alzheimer's disease. Journal of Aging Studies, 18(4), 415-428. doi: http://dx.doi.org/10.1016/ j.jaging.2004.06.005

Belleville, S. (2008). Cognitive training for persons with mild cognitive impairment. International Psychogeriatrics, 20(01), 57-66.

Bhattacharya, S., Vogel, A., Hansen, M.l., Waldorff, F.B., \& Waldemar, G. (2010). Generic and disease-specific measures of quality of life in patients with mild Alzheimer's disease. Dementia and Geriatric Cognitive Disorders, 30(4), 327-333. doi: http://dx.doi.org/10.1159/000320588 
Birks, J. (2006). Cholinesterase inhibitors for Alzheimer's disease. Cochrane Database Systematic Review. (1): CD005593.

Black, B.S., Kass, N.E., Fogarty, L.A., \& Rabins, P.V. (2007). Informed consent for dementia research: The study enrollment encounter. IRB: Ethics and Human Research, 29(4), 7-14. doi: 10.2307/30033237

Black, B.S., Rabins, P.V., Sugarman, J., \& Karlawish, J.H. (2010). Seeking assent and respecting dissent in dementia research. American Journal of Geriatric Psychiatry, 18(1), 77-85. Retrieved from http://search.proquest.com/docview/195989272?accountid=14782

Boger, J., Jackson, P., Mulvenna, M., Sixsmith, J., Sixsmith, A., Mihailidis, A., .. . Martin, S. (2016). Principles for fostering the transdisciplinary development of assistive technologies. Disability and Rehabilitation: Assistive Technology, 1-11. doi: 10.3109/17483107.2016.1151953

Borson, S., Scanlan, J., Brush, M., Vitallano, P., \& Dokmak, A. (2000). The Mini-Cog: A cognitive 'vital signs' measure for dementia screening in multi-lingual elderly. International Journal of Geriatric Psychiatry, 15(11), 1021-1027. doi: 10.1002/1099-1166(200011)15:11<1021::AID-GPS234>3.0.CO;2-6

Boulay, M., Benveniste, S., Boespflug, S., Jouvelot, P., \& Rigaud, A.S. (2011). A pilot usability study of MINWii: A music therapy game for demented patients. Technology and Health Care, 19(4), 233-246. doi: 10.3233/THC-2011-0628

Bourdieu, P. (1977). Outline of a theory of practice. Cambridge, UK: Cambridge University Press. Translated by R. Nice.

Bourdieu, P. (1990). Logic of practice. Cambridge, UK: Polity Press. Translated by R. Nice.

Boustani, M., Justiss, M.D., Frame, A., Austrom, M.G., Perkins, A.J., Cai, X., . . Hendrie, H.C. (2011). Caregiver and noncaregiver attitudes toward dementia screening. Journal of the American Geriatric Society, 59(4), 681-686. doi: 10.1111/j.15325415.2011.03327.x

Boustani, M., Perkins, A.J., Monahan, P., Fox, C., Watson, L., Hopkins, J., . . Hendrie, H.C. (2008). Measuring primary care patients' attitudes about dementia screening. International Journal of Geriatric Psychiatry, 23(8), 812-820. doi: 10.1002/gps.1983

Boustani, M., Watson, L., Fultz, B., Perkins, A.J., \& Druckenbrod, R. (2003a). Acceptance of dementia screening in continuous care retirement communities: A mailed survey. International Journal of Geriatric Psychiatry, 18(9), 780-786. doi: 10.1002/gps.918

Boustani, M., Peterson, B., Hanson, L., Harris, R., \& Lohr, K.N. (2003b). Screening for dementia in primary care: A summary of the evidence for the U.S. preventive services task force. Annals of Internal Medicine, 138(11), 927.

$\mathrm{Bpac}^{\mathrm{nz}}$. (2008). Antipsychotics in dementia: Best practice guide. Dunedin, NZ: Bpac ${ }^{\mathrm{nz}}$. Retrieved from http://www.bpac.org.nz/a4d/resources/docs /bpac_A4D_best_practice_guide.pdf

Bpac $^{\mathrm{nz}}$. (2010). The pharmacological management of Alzheimer's disease: The place of donepezil. Best Practice Journal, 30, 28-35. Retrieved from http://www.bpac.org.nz/BPJ/2010/August/alzheimers.aspx

Bpac $^{\mathrm{nz}}$. (2013). Managing patients with dementia: What is the role of antipsychotics? Best Practice Journal, 57, 26-36. Retrieved from http://bpac.org.nz/BPJ/2013/December/ docs/BPJ57.pdf

Braun, V., \& Clarke, V. (2006). Using thematic analysis in psychology. Qualitative Research in Psychology, 3(2), 77-101. doi: http://dx.doi.org/ 10.1191/ 1478088706qp063oa 
Breuil, V., de Rotrou, J., Forette, F., Tortrat, D., Ganansia-Ganem, A., \& Frambourt, A. (1994). Cognitive stimulation of patients with dementia: Preliminary results. International Journal of Geriatric Psychiatry, 9, 211-217. Retrieved from http://web.ebscohost.com/ehost/pdfviewer/ pdfviewer?sid=a163f6c9-286e-4db69429-9ed613ee280c\%40sessionmgr 113\&vid=1\&hid=118

Brickell, K. (2012). Research review educational series: Treatment of Alzheimer's disease in New Zealand. Research Review. Retrieved from http://www. researchreview.co.nz

Brod, M., Stewart, A.L., Sands, L., \& Walton, P. (1999). Conceptualization and measurement of quality of life in dementia: The dementia quality of life instrument (DQoL). The Gerontologist, 39(1), 25-36. Retrieved from http://gerontologist.oxfordjournals.org/content/39/1/25.full.pdf + html

Brooker, D. (2001). Therapeutic activity. In C. Cantley (Ed.), A handbook of dementia care (pp. 146-159). Buckingham, UK: Open University Press.

Brooker, D. (2004). What is person-centred care in dementia? Reviews in Clinical Gerontology, 13(3), 215-222. Retrieved from http://search.proquest.com.helicon. vuw.ac.nz/docview/211549769?accountid=14782

Brooker, D. (2007). Person-centred dementia: Making services better. London, UK: Jessica Kingsley Publishers.

Brooker, D., La Fontaine, J., de Vries, K., Porter, T., \& Surr, C. (2011). How can I tell you what's going on here? The development of PIECE-dem: An observational framework to bring to light the perspective of residents with advanced dementia living in care homes. Final Report to Department of Health Policy Research, Worcester, UK: Association for Dementia Studies.

Brooker, D., La Fontaine, J., de Vries, K., Latham, I. (2013). The development of PIECEdem: Focusing on the experience of care for people living with advanced dementia. Clinical Psychology Forum, 250, 38-46.

Brown, C. (2001). Descartes: Summary of some major points. Retrieved from http://www.trinity.edu/cbrown/modern/descartesMajorPoints.html

(Last update: January 9, 2001. Curtis Brown | Classical Modern Philosophy | Philosophy Department | Trinity University cbrown@trinity.edu)

Brownie, S., \& Nancarrow, S. (2013). Effects of person-centered care on residents and staff in aged-care facilities: A systematic review. Clinical Interventions in Aging, 8, 1-10. Retrieved from https://www.researchgate.net/profile/Susan

Cacchione, P.Z. (2011). People with dementia: Capacity to consent to research participation. Clinical Nurse Research, 20, 223-227. doi: $10.1177 / 1054773811415810$

Campbell, P., Wright, J., Oyebode, J., Job, D., Crome, P., Bentham, P., . . Lendon, C. (2008). Determinants of burden in those who care for someone with dementia. International Journal of Geriatric Psychiatry, 23(10), 1078-1085. doi: 10.1002/gps.2071

Cantegreil-Kallen, I., de Rotrou, J., \& Rigaud, A.S. (2009). Cognitive stimulation for people with mild cognitive impairement and early dementia. In E. Moniz-Cook \& J. Manthorpe (Eds.), Early psychological interventions in dementia (pp. 81-92). London, England: Jessica Kingsley Publishers.

Choi, J., \& Twamley, E.W. (2013). Cognitive rehabilitation therapies for Alzheimer's disease: A review of methods to improve treatment engagement and self-efficacy. Neuropsychology Review, 23(1), 48-62. doi: 10.1007/s11065-013-9227-4

Clare, L., \& Jones, R.S. (2008). Errorless learning in the rehabilitation of memory impairment: A critical review. Neuropsychology Review, 18(1), 1-23. doi:http://dx.doi.org/10.1007/s11065-008-9051-4 
Clare, L., Wilson, B.A., Carter, G., Breen, K., Gosses, A., \& Hodges, J.R. (2000). Intervening with everyday memory problems in dementia of alzheimer type: An errorless learning approach. Journal of Clinical and Experimental Neuropsychology, 22(1), 132-146. doi: 10.1076/1380-3395(200002)22:1;1$8 ;$ FT132

Clare, L., \& Wilson, B. (2004). Memory rehabilitation for people with early-stage dementia: A single case comparison of four errorless learning methods. Zeitschrift Gerontopsychologie Psychiatrie, 17, 109-117.

Clare, L., \& Woods, B. (2003/2008). Cognitive rehabilitation and cognitive training for early-stage Alzheimer's disease and vascular dementia. Cochrane Database of Systematic Reviews. 2003, Issue 4. ART.No. CD003260. Doi: $10.1002 / 14651858 . C D 003260$

Cohen, D., \& Eisdorfer, C. (1986). The loss of self: A family resource for the care of Alzheimer's disease and related disorders. London, UK: W.W. Norton.

Cohen, D., Kennedy, G., \& Eisdorfer, C. (1984). Phases of change in patients with Alzheimer's disease: A conceptual dimension of defining healthcare management. Journal of the American Geriatric Society, 32, 11-15.

Cohen, G.D., Firth, K.M., Biddle, S., Lloyd Lewis, M.J., \& Simmens, S. (2008). The first therapeutic game specifically designed and evaluated for Alzheimer's disease. Am Journal of Alzheimers Disease and Other Dementias, 23(6), 540-551. doi: $10.1177 / 1533317508323570$

Cohen-Mansfield, J (2014). Understanding behaviour. In M. Downs \& B, Bowers (Eds), Excellence in dementia care: Research into practice (2nd Ed). (pp. 220-239). Birkshire, England: UK: Open University Press.

Cohen-Mansfield, J., Creedon, M.A., Malone, T., Parpura-Gill, A., Dakheel-Ali, M., \& Heasly, C. (2006). Dressing of cognitively impaired nursing home residents: Description and analysis. The Gerontologist, 46(1), 89-96

Connell, C.M., Roberts, J.S., McLaughlin, S.J., \& Carpenter, B.D. (2009). Black and white adult family members' attitudes toward a dementia diagnosis. American Geriatric Society, 57(9), 1562-1568. doi: 10.1111/j.1532-5415.2009.02395.x

Cooke, M.L., Moyle, W., Shum, D.H.K., Harrison, S.D., \& Murfield, J.E. (2010). A randomized controlled trial exploring the effect of music on agitated behaviours and anxiety in older people with dementia. Aging Ment Health, 14(8), 905-916. doi: 10.1080/13607861003713190

Creswell, J.W. (2014). Research design: Qualitative, quantitative, and mixed methods approaches $\left(4^{\text {th }}\right.$ ed.). London, England: Sage Publications Ltd.

Cubit, K. (2010). Informed consent for research involving people with dementia: A grey area. Contemporary Nurse, 34(2), 230-236. Retrieved from http://search.informit.com.au/documentSummary;dn=249831860243966;res=IEL HEA> ISSN: $1037-6178$

Davis, D. (2004). Dementia: Sociological and philosophical constructions, Social Science Medicine, 58 (2): 369-378.

Davis, B.H., \& Shenk, D. (2015a). Technology and dementia. American Journal of Alzheimer's Disease \& Other Dementias. 30(1), 5. doi: 10.1177/153331755568943

Davis, B.H., \& Shenk, D. (2015b). Beyond reminiscence: Using generic video to elicit conversational language. American Journal of Alzheimer's Disease \& Other Dementias. 30(1), 61-68. doi: 10.1177/1533317514534759

de Bruin, N., Sacrey, L.A.R., Brown, L.A., Doan, J., \& Whishaw, I.Q. (2008). Visual guidance for hand advance but not hand withdrawal in a reach-to-eat task in adult humans: Reaching is a composite movement. Journal of Motor Behavior, 40(4), 337-346. 
Denzin, N.K., \& Lincoln, Y.S. (2003). Strategies for qualitative inquiry (2 ${ }^{\text {nd }}$ ed.). Thousand Oaks, CA: Sage Publications Inc.

Descartes, R. (1993). Meditations on first philosophy ( $3^{\text {rd }}$ ed.). Indianapolis, USA: Hackett Publishing Company Ltd. Translated by D.A. Cress.

Descartes, R (1997) Key philosophical writings. Hertfordshire, UK: Wordsworth Editions Ltd. Translated by E.S. Haldane \& G.R.T. Ross.

de Vries, K., Brooker, D.J., \& Smith, P. (2012). Dementia skills and competencies for primary care liaison: A model for improving identification and timely diagnosis. Primary Health Care Research \& Development, 14(03), 240-249. doi:10.1017/S1463423612000266

de Vries, K., Drury-Ruddlesden, J., \& Gaul, C. (2016). 'And so I took up residence': The experiences of family members of people with dementia during admission to an acute hospital unit. Dementia. doi: 10.1177/1471301216656097

de Vries, K., Fontaine, J.L., \& Brooker, D. (2010). Review of the literature: Information and educational needs of people with dementia and their family caregivers. Association for Dementia Studies. University of Worcester, UK.

DeVries, R., Ryan, K.A, Stanczyk, A., Appelbaum, P.S., Damschroder, L., Knopman, D.S., \& Kim, S.Y.H. (2013). Public's approach to surrogate consent for dementia research: Cautious pragmatism. American Journal of Geriatric Psychiatry, 21(4), 364-372. Retrieved from http://search.proquest.com/docview/1325113021? accountid $=14782$

de Werd, M.M., Boelen, D., Old Rikkert, M.G., \& Kessels, R. P. (2013). Errorless learning of everyday tasks in people with dementia. Clinical Interventions in Aging, 8, 1177.

de Werd, M.M., Boelen, D., Olde Rikkert, M.G., \& Kessels, R.P. (2015). Development and evaluation of a clinical manual on errorless learning in people with dementia. Brain Impairment, 1-12. doi: 10.1017/Brimp.2015.8

Dewing, J. (2008). Personhood and dementia: Revisiting Tom Kitwood's ideas. International Journal of Older People Nursing, 3(1), 3-13. doi: 10.1111/j.17483743.2007.00103.x

Dickson-Swift, V., James, E. L., Kippen, S., \& Liamputtong, P. (2006). Blurring boundaries in qualitative health research on sensitive topics. Qualitative Health Research, 16(6), 853-871. doi: 10.1177/1049732306287526

Dickson-Swift, V., James, E.L., Kippen, S., \& Liamputtong, P. (2007). Doing sensitive research: What challenges do qualitative researchers face? Qualitative Research, 7(3), 327-353. doi: 10.1177/1468794107078515

Dou, Z.L., Man, D.W., Ou, H.N., Zheng, J.L., \& Tam, S.F. (2006). Computerized errorless learning-based memory rehabilitation for Chinese patients with brain injury: A preliminary quasi-experimental clinical design study. Brain Injury, 20(3), 219-225. doi: 10.1080/02699050500488215

Drury-Ruddlesden, J., deVries, K., Nelson, K., \& Kernohan, E. (2017). Cognitive Stimulation Therapy using computer technology for people with moderate to severe dementia. Final Report (confidential) for the Ministry of Science and Innovation.

Drury, V., Francis, K., \& Chapman, Y. (2007). Taming the rescuer: The therapeutic nature of qualitative research interviews. International Journal of Nursing Practice, 13(6), 383-384. doi: 10.1111/j.1440-172X.2007.00654.x

Dyall, L. (2014). Dementia: Continuation of health and ethnic inequalities in New Zealand. New Zealand Medical Journal (Online), 127(1389).

Edvardsson, D., \& Innes, A. (2010). Measuring person-centered care: A critical comparative review of published tools. The Gerontologist, 50(6), 834-846. doi: 10.1093/geront/gnq047 
Ekberg, O., Hamdy, S., Woisard, V., Wuttge-Hannig, A., \& Ortega, P. (2002). Social and psychological burden of dysphagia: Its impact on diagnosis and treatment. Dysphagia, 17(2), 139-146.

Einang Alnes, R., Kirkevold, M., \& Skovdahl, K. (2011). Insights gained through Marte Meo counselling: Experiences of nurses in dementia specific care units. International Journal of Older People Nursing, 6(2), 123-132.

Enosh, G., \& Ben-Ari, A. (2016). Reflexivity: The creation of liminal spaces-researchers, participants, and research encounters. Qualitative Health Research, 26(4), 578584. doi: $10.1177 / 1049732315587878$

Evans, J.J., Levine, B., \& Bateman, A. (2004). Errorless learning. Neuropsychological Rehabilitation, 14(4), 467-476 Retrieved from http://web.b.ebscohost.com.helicon.vuw.ac.nz/ehost/pdfviewer/pdfviewer?vid=4

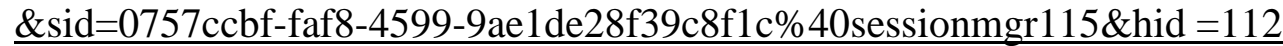

Evans, J.J., Wilson, B.A., Schuri, U., Andrade, J., Baddeley, A., Bruna, O., . . . Taussik, I. (2000). A Comparison of "errorless" and "trial-and-error" learning methods for teaching individuals with acquired memory deficits. Neuropsychological Rehabilitation: An International Journal 10(1), 67-101. Retrieved from http://www.tandfonline.com/doi/abs/10.1080/096020100389309

Favela, J. (2013). Behavior-Aware Computing: Applications and challenges. IEEE Pervasive Computing, 12(3), 14-17. doi: 10.1109/mprv.2013.44

Fereday, J., \& Muir-Cochrane, E. (2006). Demonstrating rigor using thematic analysis: A hybrid approach of inductive and deductive coding and theme development. International Journal of Qualitative Methods, 5(1), 80-92. doi: $10.1177 / 160940690600500107$

Ferland, M.B., Larente, J., Rowland, J \& Davidson, P.S.R. (2013). Errorless (re)learning of daily living routines by a woman with impaired memory and initiation: Transferrable to a new home? Brain Injury, 27(12), 1461-1469. doi: $10.3109 / 02699052.2013 .823661$

Flynn, B. (2011). "Maurice Merleau-Ponty". In Stanford Encyclopedia of Philosophy (Fall 2011 ed.), Edward N. Zalta (ed.). Retrieved from http://plato.stanford.edu/archives/fall2011/entries/merleau-ponty/

Folstein, M.F., Folstein, S.E., \& McHugh, P.R. (1975). Mini-Mental State: A practical method of grading the cognitive state of the patient for the clinician. Journal of Psychiatric Research, 12(3), 189-198. doi: http://dx.doi.org/10.1016/00223956(75)90026-6

Folstein, M.F., Folstein, S.E., White, T., \& Messer, M.A. (2010). MMSE-2: Mini-mental state examination users manual $\left(2^{\text {nd }}\right.$ ed.). USA: Psychological Assessment Resources.

Fontana, A., \& Smith, R.W. 1989. Alzheimer's disease victims: The 'unbecoming' of self and the normalization of competence. Sociological Perspectives, 32, 1, 35-46.

Forbat, L. (2003). Relationship difficulties in dementia care: A discursive analysis of two women's accounts. Dementia, 2(1), 67-84.

Freeman, E., Clare, L., Savitch, N., Royan, L., Litherland, R., \& Lindsay, M. (2005). Improving website accessibility for people with early-stage dementia: A preliminary investigation. Aging \& Mental Health, 9(5), 442-448. doi: 10.1080/13607860500142838

Fugl-Meyer, A.R., Jääskö, L., Leyman, I., Olsson, S., \& Steglind, S. (1974). The poststroke hemiplegic patient. 1. A method for evaluation of physical performance. Scandinavian Journal of Rehabilitation Medicine, 7(1), 13-31. 
Fusch, P.I., \& Ness, L.R. (2015). Are we there yet? Data saturation in qualitative research. The Qualitative Report, 20(9), 1408-1416. doi: 10.3928.01484834-201107190110.1007/s 10755-010-9142-z

Galea, M., Khan, F., Amatya, B., Elmalik, A., Klaic, M., \& Abbott, G. (2016). Implementation of a technology-assisted programme to intensify upper limb rehabilitation in neurologically impaired participants: A prospective study. Journal of Rehabilitation Medicine, 48(6), 522-528. doi: 10.2340/16501977-2087

Galbraith, B., Larkin, H., Moorhouse, A., \& Oomen, T. (2015). Intergenerational programs for persons with dementia: A scoping review. Journal of Gerontological Social Work, 58(4), 357-378. doi: 10.1080/01634372.2015. 1008166

Gee, S., Davey, J., \& Duke, J. (2001). Greying of the nation: Issues for health care in the context of an ageing population. Health Manager, 8(2), 236-240

Gelfand, T., Vick, J.C., \& Lewis, B.A. (2014). Computerized cognitive training for People with Alzheimer's disease and other dementias: A meta-analysis of the current literature. E-Hearsay, 2(4), 54-70. Retrieved from http://www.ohioslha. org/pdf/MembersOnly/Journals/eHearsay2014-SpecialWinterEdition.pdf\#page= 55

George, D.R., \& Whitehouse, P.J. (2011). Marketplace of memory: What the brain fitness technology industry says about us and how we can do better. The Gerontologist, 51(5), 590-596. doi: 10.1093/geront/gnr042

Goffman, E. (1963). Stigma: Notes on the management of spoied identity. Middlesex, England: Penguin books.

Gray, S.L., Dublin, S., Yu, O., Walker, R., Anderson, M., Hubbard, R.A., . . Larson, E.B. (2016). Benzodiazepine use and risk of incident dementia or cognitive decline: Prospective population based study. BMJ, 352. doi: 10.1136/bmj.i90

Greenwood, D.E., Gordon, C., Pavlou, C., \& Bolton, J.V. (2016). Paradoxical and powerful: Volunteers' experiences of befriending people with dementia. Dementia, O(0), 1-19. doi: 10.1177/1471301216654848

Grigorovich, A., Rittenberg, N., Dick, T., McCann, A., Abbott, A., Kmielauskas, A., . . Cameron, J.I. (2016). Roles and coping strategies of sons caring for a parent with dementia. American Journal of Occupational Therapy, 70(1), 1-9 9p. doi: 10.5014/ajot.2016.017715

Guba, E.G. (1981). ERIC/ECTJ Annual review paper: Criteria for assessing the trustworthiness of naturalistic inquiries. Educational Communication and Technology, 29(2), 75-91. Retrieved from http://www.jstor.org.helicon. vuw.ac.nz/ stable/30219811

Guest, G., Bunce, A., \& Johnson, L. (2006). How many interviews are enough? An experiment with data saturation and variability. Field methods, 18(1), 59-82. doi: $10.1177 / 1525822$ X05279903

Guillemin, M., \& Gillam, L. (2004). Ethics, reflexivity, and "Ethically Important Moments" in research. Qualitative Inquiry, 10(2), 261-280. doi: $10.1177 / 1077800403262360$

Hale, A.L., Satherley, J.A., McMillan, N.J., Milosavljevic, S., Hijmans, J.M., \& King, M.J. (2012). Participant perceptions of use of CyWee $\mathrm{Z}$ as adjunct to rehabilitation of upper-limb function following stroke. Journal of Rehabilitation Research and Development, 49(4), 623-634. doi:http://dx.doi.org/10.1682/JRRD. 2001.04.0070

Hammar, L.M., Emami, A., Götell, E., \& Engström, G. (2011). The impact of caregivers' singing on expressions of emotion and resistance during morning care situations in persons with dementia: An intervention in dementia care. Journal of Clinical Nursing, 20(7/8), 969-978. doi: 10.1111/j.1365-2702.2010.03386.x 
Hansebo, G., \& Kihlgren, M. (2001). Carers' reflections about their video-recorded interactions with patients suffering from severe dementia. Journal of Clinical Nursing, 10(6), 737-747.

Hansen, R.A., Gartlehner, G., Webb, A.P., Morgan, L.C., Moore, C.G., \& Jonas, D.E. (2008). Efficacy and safety of donepezil, galantamine, and rivastigmine for the treatment of Alzheimer's disease: A systematic review and meta-analysis. Clinical Interventions in Aging, 3(2), 211.

Hatfield, C (2014). René Descartes. In Stanford Encyclopedia of Philosophy. Retrieved from http://plato.stanford.edu/entries/descartes/

Hayman, K. J., Kerse, N., Dyall, L., Kepa, M., Teh, R., Wham, C., . . Jatrana, S. (2012). Life and Living in Advanced Age: A cohort study in New Zealand -Te Puāwaitanga o Nga Tapuwae Kia Ora Tonu, LiLACS NZ: Study protocol. BMC Geriatrics, 12(1), 1-13. doi: 10.1186/1471-2318-12-33

Health and Disability Commissioner. (1996). The Code of Health and Disability Services Consumers' Rights. Auckland, NZ: Author.

Heyn, P.C., Cassidy, J.L., \& Bodine, C. (2015). The Rehabilitation Engineering Research Center for the Advancement of Cognitive Technologies. American Journal of Alzheimers Disease and Other Dementias, 30(1), 6-12. doi: $10.1177 / 1533317514531444$

Hijmans, J.M., Hale, L.A., Satherley, J.A., McMillan, N.J., \& King, M.J. (2011). Bilateral upper-limb rehabilitation after stroke using a movement-based game controller. Journal of Rehabilitation Research and Development, 48(8), 1005-1013. doi: GW15523V21243741 [pii] 10.3233/NRE-2009-0480

Houghton, C., Casey, D., Shaw, D., \& Murphy, K. (2013). Rigour in qualitative case-study research. Nurse Researcher, 20(4), 12-17. doi:http://dx.doi.org/ 10.4135/9781412957397.n347

Huber, B. A. (2003). On the terminology of polytomies. Cladistics, 19(3), 273-273. doi: 10.1111/j.1096-0031.2003.tb00371.x

IBM Corp.(2013). IBM SPSS Statistics for Windows, Version 22.0. Armonk, NY: IBM Corp.

Iliffe, S., Jain, P., \& Wilcock, J. (2009). Recognition of and response to dementia syndrome in primary care: Part 1. InnovAiT 2, 230-36

Iliffe, S., \& Manthorpe, J. (2004). The recognition and response to dementia in the community: Lessons for professional development. Learning in Health and Social Care, 3, 5-16.

Iliffe, S., Manthorpe, J., \& Eden, A. (2003). Sooner or later? Issues in the early diagnosis of dementia in general practice: A qualitative study. Family Practice, 20, 376-81.

Iliffe, S., \& Orrell, M. (2006). Identifying unmet health needs in older people: Comprehensive screening is not the answer. British Journal of General Practice, 56, 404-406.

Iliffe, S., \& Wilcock, J. (2005). The identification of barriers to the recognition of, and response to dementia in primary care using a modified focus group approach. Dementia, 4, 73-85.

Iliffe, S., Wilcock, J., \& Haworth, D. (2006). Obstacles to shared care for patients with dementia: A qualitative study. Family Practice, 23(3): 353-362. doi: 10.1093/fampra/cmi116

Im-Able Ltd (2013), Im-Able clinic based exercise system for stroke recovery: Accelerated stroke recovery: Able-X Pro edition user manual ( $1^{\text {st }}$ ed. $)$;. Lower Hutt, New Zealand: Author. 
Jack, S. (2008). Guidelines to support nurse-researchers reflect on role conflict in qualitative interviewing. The Open Nursing Journal, 2, 58-62. doi: $10.2174 / 1874434600802010058$

Jokel, R., \& Anderson, N.D. (2012). Quest for the best: effects of errorless and active encoding on word re-learning in semantic dementia. Neuropsychological Rehabilitation, 22(2), 187-214. Retrieved from http://www.ncbi.nlm. nih.gov/pubmed/22250922 doi:10.1080/09602011.2011.639626

Jokel, R., Rochon, E., \& Anderson, N.D. (2010). Errorless learning of computer-generated words in a patient with semantic dementia. Neuropsychological Rehabilitation, 20(1), 16-41. doi: 10.1080/09602010902879859

Jonas, C., Schiffczyk, C., Lahmeyer, C., Mueller, F., \& Riepe, M.W. (2011). Staging dementia using proxy-reported activities of daily living. Dementia and Geriatric Cognitive Disorders, 32(2), 111-117. doi: http://dx.doi.org/10.1159/000331420

Jones, R.S.P, \& Eayrs, C.B. (1992). The use of errorless learning procedures in teaching people with a learning disability: A critical review. Mental Handicap Research, 5, 204-212.

Jootun, D., McGhee, G., \& Marland, G.R. (2009). Reflexivity: Promoting rigour in qualitative research. Nursing Standard. 23(23), 42-46. http://go.galegroup. com.helicon.vuw.ac.nz

Jordan, K., Sampson, M., \& King, M. (2014). Gravity-supported devices with computer gaming improves arm function in chronic stroke. Archives of Physical Medicine and Rehabilitation, 95(8), 1484-1489. doi: 10.1016/j.apmr.2014.02.028

Kawashima, R., Okita, K., Yamazaki, R., Tajima, N., Yashida, H., Taira, M., . . . Sugimoto, K. (2005). Reading aloud and arithmetic calculation improve frontal function of people with dementia. Journals of Gerontology, 60 A(3), 380-384.

Keady, J., \& Nolan, M. (1995). IMMEL 2: Working to augment coping responses in early dementia. British Journal of Nursing, 4, 377-380.

Keller, F.S, \& Schoenfeld, W.N. (1950). Principles of psychology: A systematic text in the science of behavior. New York, Appleton-Century-Crofts. Retrieved from https://archive.org/details/principlesofpsyc032635mbp

Kerse N., LiLACS NZ. (2014a). Extra help with daily activities in advanced age: Findings from LiLACS NZ. Auckland. School of Population Health, University of Auckland. Release: $\quad 10 \quad$ November 2015. Retrieved from: https://www.fmhs.auckland.ac.nz/en/faculty/lilacs/research/publications.html

Kerse N., LiLACS NZ. (2014b). Relationships and emotional support in advanced age: Findings from LiLACS NZ. Auckland. School of Population Health, University of Auckland. Release: 22 September $2015 . \quad$ Retrieved from: https://www.fmhs.auckland.ac.nz/en/faculty/lilacs/research/publications.html

Khan, F., Amatya, B., Elmalik, A., Lowe, M., Ng, L., Reid, I., \& Galea, M.P. (2016). An enriched environmental programme during inpatient neuro-rehabilitation: A randomized controlled trial. Journal of Rehabilitation Medicine, 48(5), 417-425. doi: 10.2340/16501977-2081

Khan, S. \& VanWynsberghe, R. (2008), Cultivating the under-mined: Cross-case analysis as knowledge mobilization. Forum Qualitative Sozialforschung/Forum: Qualitative Social Research, 9(1), Art. 34, http://nbnresolving.de/um:nbn:de:D114-fqs0801348.

Kihlstrom, J.F., Dorfman, J., \& Park, L. (2007). Implicit and explicit memory and learning. Retrieved from http://socrates.berkeley.edu/ kihlstrm/ IandMLandM.htm

King, M., Hale, L., Pekkari, A., Persson, M., Gregorsson, M., \& Nilsson, M. (2010). An affordable, computerized, table-based exercise system for stroke survivors. 
Disability and Rehabilitation Assistive Technology, 5(4), 288-293. doi: 10.3109/17483101003718161

King, M., Hijmans, J.M., Sampson, M., Satherley, J., \& Hale, L. (2012). Home-based stroke rehabilitation using computer gaming. New Zealand Journal of Physiotherapy, 40(3), 128-134.

Kimchi, J., Polivka, B., \& Stevenson, J.S. (1991). Triangulation: Operational definitions. Nursing Research, 40(6), 364-366.

Kirkman, A. (2011). Caring 'from duty and the heart': Gendered work and Alzheimer's disease. Women's Studies Journal, 25(1), 1-16.

Kitwood T. (1989). Brain, mind and dementia: With particular reference to Alzheimer's disease. Ageing and Society 9(1), 1-15. doi:10.1017/S0144686X00013337

Kitwood T. (1990). The dialetics of dementia: with particular reference to Alzheimer's disease. Aging and Society 10(2), 177-196. doi:10.1017/S0144686X00008060

Kitwood, T. (1993a). Person and process in dementia. International Journal of Geriatric Psychiatry, 8(7), 541-545. Retrieved from: http://helicon.vuw.ac.nz/ login?url=http://search.ebscohost.com /login.aspx?direct=true \&db=a2h\&AN $=12102314 \&$ site $=$ ehost-live

Kitwood, T. (1993b). Towards a theory of dementia care: The interpersonal process. Ageing \& Society, 13(01), 51-67. doi:10.1017/S0144686X00000647

Kitwood, T.M. (1997). Dementia reconsidered: The person comes first. Berkshire, UK: Open University Press.

Kontos, P.C. (2003). "The painterly hand": Embodied consciousness and Alzheimer's disease. Journal of Aging Studies, 17, 151-170.

Kontos, P.C (2004). Ethnographic reflections on selfhood, embodiment and Alzheimer's disease. Ageing and Society, 24, 829-849. doi:10.1017/S0144686X04002375

Kontos, P.C (2005). Embodied selfhood in Alzheimer's disease: Rethinking person centred care. Dementia, 4(4), 553-570. doi: 10.1177/1471301205058311

Kontos, P.C. (2012). Rethinking sociability in long-term care: An embodied dimension of selfhood. Dementia, 11(3), 329-346. doi: 10.1177/1471301211421073

Kontos, P.C. (2014), Selfhood and the body in dementia care. In Downs, M \& Bowers, B (Eds.), Excellence in dementia care research into practice ( $2^{\text {nd }}$ ed.), pp.122-311. Berkshire, England: Open University Press.

Kontos, P.C., \& Martin, W. (2013). Exploring critical narratives of selfhood, surveillance, and dementia care. Dementia, 12(3), 288-302. doi: 10.1177/1471301213479787

Kontos, P., Miller, K.-L., Mitchell, G. J., \& Stirling-Twist, J. (2015). Presence redefined: The reciprocal nature of engagement between elder-clowns and persons with dementia. Dementia, 0(0), 1-21. doi: 10.1177/1471301215580895

Kontos, P.C., \& Naglie. G. (2007a). Bridging theory and practice: Imagination, the body, and person-centred dementia care. Dementia, 6(4), 549-569. doi: $10.1177 / 1471301207084394$

Kontos, P.C., \& Naglie, G. (2007b). Expressions of Personhood in Alzheimer's Disease: An evaluation of research-based theatre as a pedagogical tool. Qualitative Health Research, 17(6), 799-81. doi:10.1177/ 1049732307302838

Kontos, P.C., \& Naglie, G (2009). Tacit knowledge of caring and embodied selfhood. Sociology of Health \& Illness, 31(5), 688-704. doi: 10.1111/j.14679566.2009.01158.x

Kørner, A., Lauritzen, L., Abelskov, K., Gulmann, N., Brodersen, A.M., WedervangJensen, T., \& Kjeldgaard, K.M. (2006). The Geriatric Depression Scale and the Cornell Scale for Depression in Dementia. A validity study. Nordic Journal of Psychiatry, 60(5), 360-364. doi: 10.1080/08039480600937066 
Koshibu, K. (2016). Nootropics with potential to (re) build neuroarchitecture. Neural regeneration research, 11(1), 79. doi: 10.4103/1673-5374.169635

Kueider, A.M., Parisi, J.M., Gross, A.L., \& Rebok, G.W. (2012). Computerized cognitive training with older adults: A systematic review. PLoS ONE, 7(7), 1-13. doi: 10.1371/journal.pone.0040588

Lacy, M., Kaemmerer, T., \& Czipri, S. (2015). Standardized Mini-Mental State Examination Scores and verbal memory performance at a memory center: Implications for cognitive screening. American Journal of Alzheimers Disease and other Dementias, 30(2), 145-152. doi: 10.1177/1533317514539378

Laffan, A.J., Metzler-Baddeley, C., Walker, I., \& Jones, R.W. (2010). Making errorless learning more active: Self-generation in an error free learning context is superior to standard errorless learning of face-name associations in people with Alzheimer's disease. Neuropsychological Rehabilitation, 20(2), 197-211. doi:10.1080/09692010903202432

Lai, S.W., Lin, C.H., Liao, K.F., Su, L.T., Sung, F.C., \& Lin, C.C. (2012). Association between polypharmacy and dementia in older people: A population-based casecontrol study in Taiwan. Geriatrics \& Gerontology International, 12(3), 491-498. doi: 10.1111/j.1447-0594.2011.00800.x

Lau, D.T., Mercaldo, N.D., Harris, A.T., Trittschuh, E., Shega, J., \& Weintraub, S. (2010). Polypharmacy and potentially inappropriate medication use among communitydwelling elders with dementia. Alzheimer Disease and Associated Disorders, 24(1), 56-63. doi:10.1097/WAD.0b013e31819d6ec9.

Lau, K.M., Parikh, M., Harvey, D.J., Huang, C.J., \& Farias, S.T. (2015). Early cognitively based functional limitations predict loss of independence in instrumental activities of daily living in older adults. Journal of the International Neuropsychological Society, 21(9), 688-698. doi: 10.1017/S1355617715000818

Lincoln, Y.S. (1992). Sympathetic connections between qualitative methods and health research. Qualitative Health Research, 2(4), 375-391.

Lincoln, Y.S. \& Guba, E.G. (2000).Paradigmatic controversies, contradictions, and emerging confluences. In N.K. Denzin \& Y.S. Lincoln (Eds), Handbook of qualitative research ( $\left.2^{\text {nd }} \mathrm{Ed}\right)$ (pp163-188): London, England: Sage Publications.

Link, B.G., \& Phelan, J.C. (2001). Conceptualizing stigma. Annual Review of Sociology, 27, 363-385.

Logsdon, R. (1996). Quality of life-AD. Washington, USA: University of Washington.

Logsdon, R.G., Gibbons, L.E., McCurry, S.M., \& Teri, L. (2002). Assessing quality of life in older adults with cognitive impairment. Psychosomatic Medicine, 64(3), 510519.

MacPhillamy, D.J., \& Lewinsohn, P.M. (1982). The pleasant events schedule: Studies on reliability, validity, and scale intercorrelation. Journal of Consulting and Clinical Psychology, 50(3), 363-380.

Martin, R., \& Paki, P. (2012). Towards inclusion: The beginnings of a bicultural model of dementia care in Aotearoa New Zealand. Dementia, 11(4), 545-552. doi: $10.1177 / 1471301212437821$

McArthur, G. (2016, April 23). Alzheimer's disease breakthrough: Melbourne drug trial achieves amazing results. Herald Sun. Retrieved from http://www.heraldsun.com.au/news/alzheimers-disease-breakthrough-melbournedrug-trial-achieves-amazing-results/newsstory/d96671bc643b16d2694e85e0639fea3d

McShane, R., Areosa Sastre, A., \& Minakaran, N. (2006). Memantine for dementia. The Cochrane Database of Systematic Reviews. Issue 2. Art. No.: CD003154. doi: 10.1002/14651858.CD003154.pub5. 
Medsafe (2014a). New Zealand datasheet: Donepezil. Retrieved from http://www.medsafe.govt.nz/profs/datasheet/d/donepeziltab.pdf

Medsafe (2014b). Paroxetine. Retrieved from http://www.medsafe.govt.nz/profs/ datasheet/p/Paroxetinemesy latetab.pdf

Medsafe (2014c). Consumer medicine information: Arrow-Amitriptyline. Retrieved from http://www.medsafe.govt.nz/profs/datasheet/a/ArrowAmitryptylinetab.pdf

Medsafe (2014d). New Zealand datasheet: Antinaus: Prochlorperazine maleate tablets 5mg. Retrieved from http://www.medsafe.govt.nz/profs/datasheet/a/ Antinaustab.pdf

Medsafe. (2014e). New Zealand datasheet: Arrow-Citalopram (Citalopram hydrobromide tablets). Retrieved from http://www.medsafe.govt.nz/ profs/datasheet/a/ArrowCitalopramtab.pdf

Medsafe (2015a). New Zealand datasheet: Apo-Risperidone. Retrieved from http://www.medsafe.govt.nz/profs/datasheet/a/ApoRisperidonetaboralsoln.pdf

Medsafe (2015b). New Zealand datasheet: Quetapel (as quetiapine fumarate). Retrieved from http://www.medsafe.govt.nz/profs/datasheet/s/ seroqueltab.pdf

Medsafe. (2015c). New Zealand datasheet: Ativan (Lorazepam). Retrieved from http://www.medsafe.govt.nz/profs/datasheet/a/Ativantab.pdf

Mehmet, M., \& Akin, P. (2003). Type I error rate and power of three normality tests. Pakistan Journal of Information Technology, 2(2), 135-139.

Mehta, C.R., \& Patel, N.R. (2012 ). IBM SPSS Exact Tests. Cambridge, Massachusetts: Cytel Software Corporation and Harvard School of Public Health.

Merleau-Ponty, M. (2014). Phenomenology of perception. London, England: Routledge. Translated by D. A. Landes.

Metzler-Baddeley, C., \& Snowden, J.S. (2005). Brief report: Errorless versus errorful learning as a memory rehabilitation approach in Alzheimer's disease. Journal of Clinical and Experimental Neuropsychology, 27(8), 1070-1079. doi: 10.1080/13803390490919164

Miles, M., \& Hubberman, A. (1994). Qualitative data analysis ( $\left.2^{\text {nd }} \mathrm{ed}\right)$. Thousand Oaks, CA: Sage Publications.

Millett, S. (2011). Self and embodiment: A bio-phenomenological approach to dementia. Dementia, O(0), 1-14. doi: 10.1177/1471301211409374

Ministry of Health. (2013a). The health of Mãori adults and children. Retrieved from http://www.health.govt.nz/system/files/documents/publications/health-maoriadults-children-summary.pdf

Ministry of Health. (2013b). New Zealand Dementia Care Pathway Framework (Final Draft).Retrieved from http://ndc.hiirc.org.nz/page/38506/final-draft-of-the-nznational-dementia-care/?tab=5328\&section=19790: Author.

Missotten, P., Squelard, G., Ylieff, M., Di Notte, D., Paquay, L., De Lepeleire, J., . . . Fontaine, O. (2008). Relationship between quality of life and cognitive decline in dementia. Dementia and Geriatric Cognitive Disorders, 25(6), 564-572.

Mitchell, G., \& Agnelli, J. (2015). Person-centred care for people with dementia: Kitwood reconsidered. Nursing Standard,30(7), 46-50. doi: /10.7748/ns.30. 7.46.s47

Mittelman, M.S., Roth, D.L., Haley, W.E., \& Zarit, S.H. (2004). Effects of a caregiver intervention on negative caregiver appraisals of behavior problems in patients with Alzheimer's disease: Results of a randomized trial. Journals of Gerontology Series B: Psychological Sciences and Social Sciences, 59(1), P27-P34.

Mole, B. (2016). Billion-dollar brain training industry a sham-nothing but placebo, study suggests. arsTechnica, 1-7. Retrieved from: http://arstechnica.com/science /2016/06/billion-dollar-brain-training-industry-a-sham-nothing-but-placebostudy-suggests/ 
Moniz-Cook, E., \& Manthorpe, J. (Eds.). (2009). Early Psychological Interventions in Dementia: Evidence-Based Practice. London, England: Jessica Kingsley Publishers.

Morhardt, D., \& Spira, M. (2013). From person-centered care to relational-centered care. Generations, 37(3), 37-44.

Morse, W.C., Lowery, D.R., \& Steury, T. (2014). Exploring saturation of themes and spatial locations in qualitative public participation geographic information systems research. Society \& Natural Resources, 27(5), 557-571. doi: 10.1080/08941920.2014.888791

Moyle, W., Murfield, J.E., Griffiths, S.G., \& Venturato, L. (2012). Assessing quality of life of older people with dementia: A comparison of quantitative self-report and proxy accounts. Journal of Advanced Nursing, 68(10), 2237-2246. doi: 10.1111/j.1365-2648.2011.05912.x

National Ethics Advisory Committee. (2012). Ethical guidelines for observational studies: Observational research, audits and related activities (revised ed.). Wellington: Ministry of Health. Retrieved from http://neac.health.govt.nz/streamlined-ethicalguidelines-health-and-disability-research

New Zealand Medical Council. (2011). Information, choice of treatment and informed consent. www.menz.org.nz

NICE-SCIE (National Institute for Clinical Excellence). (2006/2015). Dementia: Supporting people with dementia and their carers in health and social care: Clinical guideline. 42. London, England: Author. Retrieved from http://www.nice.org.uk/guidance/cg42

Nolan, L., McCarron, M., McCallion, P., \& Murphy-Lawless, J. (2006a). Perceptions of stigma in dementia: An exploratory study. Alzheimer Society of Ireland: School of Nursing and Midwifery, Trinity College Dublin. Retrieved from: http://www.lenus.ie/hse/handle/10147/299918\#

Nolan, M., Davies, S., \& Brown, J. (2006b). Transitions in care homes: Towards relationship-centred care using the "Senses Framework". Quality in Ageing Policy, Practice and Research, 7(3), 5-14. doi:10.1108/14717794200600015

Nolan, M., Ryan, T., Enderby, P., \& Reid, D. (2002). Towards a more inclusive vision of dementia care practice and research. Dementia, 1(2), 193-211. doi: $10.1177 / 147130120200100206$

Norberg, A., \& Hirschfeld, M. (1987). Feeding of severely demented patients in institutions: interviews with caregivers in Israel. Journal of Advanced Nursing, 12(5), 551-557. doi: 10.1111/j.1365-2648.1987.tb03044.x

Norberg, A., Backstrom, A., Athlin, E., \& Norberg, B. (1988). Food refusal amongst nursing home patients as conceptualized by nurses' aids and enrolled nurses: An interview study. Journal of Advanced Nursing, 13(4), 478-483. doi: 10.1111/j.1365-2648.1988.tb02853.x

Olde Rikkert, M.G.M., Tona, K.D., Janssen, L., Burns, A., Lobo, A., Robert, P., . . . Waldemar, G. (2011). Validity, reliability, and feasibility of clinical staging scales in dementia: A Systematic Review. American Journal of Alzheimers's Disease and Other Dementias, 26(5), 357-365. doi: 10.1177/1533317511418954

Onder, G., Zanetti, O., Giacobini, E., Frisoni, G.B., Bartorelli, L., Carbone, G., . . . Bernabei, R. (2005). Reality orientation therapy combined with cholinesterase inhibitors in Alzheimer's disease: Randomised controlled trial. British Journal of Psychiatry, 187(5), 450-455. doi: 10.1192/bjp.187.5.450

Onwuegbuzie, A.J., \& Leech, N.L. (2005). On becoming a pragmatic researcher: The importance of combining quantitative and qualitative research methodologies. 
International Journal of Social Research Methodology, 8(5), 375-387. doi: 10.1080/13645570500402447

Oremus, M., Perrault, A., Demers, L., \& Wolfson, C. (2000). Review of outcome measurement instruments in Alzheimer's disease drug trials: Psychometric properties of global scales. Journal of Geriatric Psychiatry and Neurology, 13(4), 197-205. doi: 10.1177/089198870001300404

Overton, E., Appelbaum, P.S., Fisher, S.R., Dohan, D., Roberts, L.W., \& Dunn, L.B. (2013). Alternative decision-makers' perspectives on assent and dissent for dementia research. American Journal of Geriatric Psychiatry, 21(4), 346-354. Retrieved from http://search.proquest.com/docview/1325124729? accountid=14782 doi:http:/dx.doi.org/10.1016/j.jagp.2013.01.027

Owen, A.M., Hampshire, A., Grahn, J.A., Stenton, R., Dajani, S., Burns, A.S., . . Ballard, C.G. (2010). Putting brain training to the test. Nature, 465(7299), 775-778.

Oyebode, J.R., \& Parveen, S. (2016). Psychosocial interventions for people with dementia: An overview and commentary on recent developments. Dementia, O(0), 1-28. doi: $10.1177 / 1471301216656096$

Pallant, J. (2011). SPSS survival manual: A step by step guide to data analysis using SPSS ( $4^{\text {th }}$ ed.). NSW, Australia: Allen \& Unwin.

Pasman, H.R.W., The, B.A.M., Onwuteaka-Philipsen, B.D., van der Wal, G., \& Ribbe, M.W. (2003). Feeding nursing home patients with severe dementia: A qualitative study. Journal of Advanced Nursing, 42(3), 304-311. Retrieved from: http://onlinelibrary.wiley.com/doi/10.1046/j.1365-2648.2003.02620.x/full

Passalacqua, S.A., \& Harwood, J. (2012). VIPS communication skills training for paraprofessional dementia caregivers: An intervention to increase person-centered dementia care. Clinical Gerontologist, 35(5), 425-445. doi: 10.1080/07317115.2012.702655

Pearce, A., Clare, L., \& Pistrang, N. (2002). Managing sense of self: Coping in the early stages of Alzheimer's disease. Dementia, 1(173-192). Retrieved from http://dem.sagepub.com.helicon.vuw.ac.nz/content/1/2/173.full.pdf+html

Pérès, K., Helmer, C., Amieva, H., Orgogozo, J.M., Rouch, I., Dartigues, J.F., \& Barberger-Gateau, P. (2008). Natural history of decline in instrumental activities of daily living performance over the 10 years preceding the clinical diagnosis of dementia: A prospective population-based study. Journal of the American Geriatrics Society, 56(1), 37-44.

Perkins, C. (2013). Dementia what you need to know: A guide for people with dementia and their care givers. Auckland, NZ: Random House New Zealand Ltd.

Perneczky, R., Wagenpfeil, S., Komossa, K., Grimmer, T., Diehl, J., \& Kurz, A. (2006). Mapping scores onto stages: Mini-mental state examination and clinical dementia rating. American Journal of Geriatric Psychiatry, 14(2), 139-144.

Pharoo, K. (2006). Nursing research: Principles, processes and issues ( $2^{\text {nd }}$ ed.). Baskinstoke, UK: Palgrave MacMillan.

Polit, D.F., Beck, C.T., \& Hungler, B.P. (2001). Essentials of nursing research: Methods, appraisal and utilization ( $5^{\text {th }}$ ed.). Philadelphia: USA: Lippincott.

Prince, M., Albanese, E., Guerchet, M., \& Prina, M. (2014). World Alzheimer Report 2014. Dementia and risk reduction: An analysis of protective and modifiable factors. London, England: Alzheimer's Disease International.

Prince, M., Bryce, R., \& Ferri, C. (2011). World Alzheimer Report 2011: The benefits of early diagnosis and intervention. Retrieved from http://www.alz.co.uk/research/WorldAlzheimerReport2011.pdf

Prince, M., Prina, M., \& Guerchet, M. (2013). World Alzheimer report 2013: Journey of caring. London, UK: Alzheimer's Disease International (ADI). Retrieved from 
https://scholar.google.co.nz/scholar?q=prince\%2C+world+alzheimer\%27s+report $+2013 \&$ hl $=$ en\&as_sdt $=2005 \&$ sciodt $=0 \% 2$ C5\&cites $=9025073741967785902 \&$ sci $\mathrm{psc}=\& \mathrm{oq}=$

Prince, M., Wimo, A., Guerchet, M., Ali, G., Wu, Y., \& Prina, M. (2015). World Alzheimer Report 2015. The global impact of dementia. An analysis of prevalence, incidence, cost and trends. London, England: Alzheimer's Disease International.

Reidpath, D., Chan, K., Gifford, S. and Allotey, P. (2005). He hath the French pox:

Stigma, social value and social exclusion. Sociology of Health and Illness, 27(4): 468-489.

Reisberg, B. (1988). Functional assessment staging (FAST). Psychopharmacology Bulletin, 24:653-659.

Reisberg, B. (2007). Global measures: utility in defining and measuring treatment response in dementia. International Psychogeriatrics, 19(03), 421-456. doi:10.1017/S1041610207005261.

Reisberg, B., Kenowsky, S., Franssen, E.H., Auer, S.R., \& Souren, L.E.M. (1999). Towards a science of Alzheimer's disease management: A model based upon current knowledge of retrogenesis. International Psychogeriatrics, 11(01), 7-23. doi:10.1017/S1041610299005554

Requenta, C., Ibor, M.I.L., Maestú, F., Campo, P., Ibor, J.J.L., \& Ortiz, T. (2004). Effects of cholinergic drugs and cognitive training on dementia. Dementia and Geriatric Cognitive Disorders, 18(1), 50-54.

Ridder, H.M., Wigram, T., \& Ottesen, A.M. (2009). A pilot study on the effects of music therapy on frontotemporal dementia -- developing a research protocol. Nordic Journal of Music Therapy, 18(2), 103-132 130p. doi: 10.1080/08098130903062371

Robinson, C.A., Bottorff, J.L., Pesut, B., Oliffe, J.L., \& Tomlinson, J. (2014). The male face of caregiving: A scoping review of men caring for a person with dementia. American Journal of Men's Health, 8(5), 409-426. doi: $10.1177 / 1557988313519671$

Robson, C. (2002). Real world research (2 ${ }^{\text {nd }}$ ed.). Oxford, England: Blackwell Publishing.

Rodda, J., \& Carter, J. (2012). Cholinesterase inhibitors and memantine for symptomatic treatment of dementia. BMJ, 344. doi: 10.1136/bmj.e22986

Røsvik, J., Brooker, D., Mjorud, M., \& Kirkevold, Ø. (2013). What is person-centred care in dementia? Clinical reviews into practice: The development of the VIPS practice model. Reviews in Clinical Gerontology, 23(2), 155-163. doi: http://dx.doi.org/10.1017/S0959259813000014

Røsvik, J., Kirkevold, M., Engedal, K., Brooker, D., \& Kirkevold, Ø. (2011). A model for using the VIPS framework for person-centred care for persons with dementia in nursing homes: A qualitative evaluative study. International Journal of Older People Nursing, 6(3), 227-236. doi: 10.1111/j.1748-3743.2011.00290.x

Sabat, S.R (2001). The experience of Alzheimer's disease: Life through a tangled veil. Oxford, UK: Wiley-Blackwell.

Sabat, S.R (2014). A bio-psycho-social approach to dementia. In M. Downs \& B, Bowers (Eds), Excellence in dementia care: Research into practice (2nd Ed). (pp. 107121). Birkshire, England: UK: Open University Press.

Sampson, M., Shau, Y., \& King, M.J. (2012). Bilateral upper limb trainer with virtual reality for post-stroke rehabilitation: Case series report. Disability \& Rehabilitation: Assistive Technology, 7(1), 55-62. doi:10.3109/17483107 .2011 .562959 
Sandelowski, M. (2010). What's in a name? Qualitative description revisited. Research in Nursing \& Health, 33(1), 77-84. http://onlinelibrary.wiley. com/doi/10.1002/nur.20362/abstract

Sandelowski, M., \& Leeman, J. (2012). Writing usable qualitative health research findings. Qualitative Health Research, 22(10), 1404-1413.

Santibáñez, M., Bolumar, F., \& García, A.M. (2007). Occupational risk factors in Alzheimer's disease: A review assessing the quality of published epidemiological studies. Occupational and Environmental Medicine, 64(11), 723-732. doi: 10.1136/oem.2006.028209

Schmand, B., \& Cees, L.J.L.M.. (1995). What is a significant score change on the minimental state examination? International Journal of Geriatric Psychiatry, 10(5), 411-414.

Schmitz, X., Bier, N., Joubert, S., Lejeune, C., Salmon, E., Rouleau, I., \& Meulemans, T. (2014). The benefits of errorless learning for serial reaction time performance in alzheimer's disease. Journal of Alzheimer's Disease, 39(2), 287-300. doi: 10.3233/JAD-130887

Schreibman, L., \& Koegel, R. (1981). A guideline for planning behavior modification programs for autistic children. In S.M. Turner, Calhoun, K.S. Adams, H.E. (Ed.), Handbook of clinical behavior therapy (pp. 500-526). New York, USA: Wiley.

Selwood, A., Thorgrimsen, L., \& Orrell, M. (2005). Quality of life in dementia:A one-year follow-up study. International Journal of Geriatric Psychiatry, 20, 232-237. doi: DOI: $10.1002 /$ gps.127

Shankar, K.K., Walker, M., Frost, D., \& Orrell, M.W. (1999). The development of a valid and reliable scale for rating anxiety in dementia (RAID). Aging and Mental Health, $3(1), 39-49$.

Siberski, J., Shatil, E., Siberski, C., Eckroth-Bucher, M., French, A., Horton, S., . . Rouse, P. (2015). Computer-based cognitive training for individuals with intellectual and developmental disabilities: Pilot study. American Journal of Alzheimers Disease and other Dementias, 30(1), 41-48. doi: 10.1177/1533317514539376

Sixsmith, A., Stilwell, J., \& Copeland, J. (1993). 'Rementia': Challenging the limits of dementia care. International Journal of Geriatric Psychiatry, 8(12), 993-1000.

Sidman, M. (2010). Errorless learning and programmed instruction: the myth of the learning curve. European Journal of Behavior Analysis, 11(2), 167-180. Retrieved from http://www.ejoba.org/PDF/2010_2/Sidman_2010.pdf

Siegel, S. (1957). Nonparametric statistics. The American Statistician, 11(3), 13-19.

Simons, H. (2009). Case study Research in practice. London: UK. Sage publications Ltd. Small, G.W., Rabins, P.V., Barry, P.P., Buckholz, N.S., DeKosky, S.T., Ferris, S.H., . . Oakley, F. (1997). Diagnosis and treatment of Alzheimer disease and related disorders: Consensus statement of the American Association for Geriatric Psychiatry, the Alzheimer's Association, and the American Geriatrics Society. JAMA, 278(16), 1363-1371. doi: 10.1001/jama.1997.03550160083043

Smith, S., Lamping, D.L., Banerjee, S., Harwood, R., Foley, B., Smith, P., . . Knapp, M. (2005). Measurement of health-related quality of life for people with dementia: development of a new instrument (DEMQOL) and an evaluation of current methodology. Health Technology Assessment, 9(10). Retrieved from http://www.hta.ac.uk

Smith, S., Lampling, D., Banerjee, S., Harwood, R., Foley, B., Smith, P., . . Knapp, M. (2007). Development of a new measure of health-related quality of life for people with dementia: DEMQOL. Psycholog Medicne, 37, 737-746.

Snowden, M., Sato, K., \& Roy-Byrne, P. (2003). Assessment and treatment of nursing home residents with depression or behavioral symptoms associated with dementia: 
A review of the literature. Journal of the American Geriatrics Society, 51(9), 13051317.

Son, G.R., Therrien, B., \& Whall, A. (2002). Implicit memory and familiarity among elders with dementia. Journal of Nursing Scholarship, 34(3), 263-267.

Span, M., Hettinga, M., Vernooij-Dassen, M., Eefsting, J., \& Smits, C. (2013). Involving people with dementia in the development of supportive IT applications: A systematic review. Ageing Research Reviews, 12(2), 535-551. doi: 10.1016/j.arr.2013.01.002

Spector, A., Davies, S., Woods, B., \& Orrell, M. (2000). Reality orientation for dementia: A systematic review of the evidence of effectiveness from randomized controlled trials. The Gerontologist, 40(2), 206-212. doi: 10.1093/geront/40.2.206

Spector, A., \& Orrell, M. (2006). Quality of Life (QoL) in dementia: A comparison of the perceptions of people with dementia and care staff in residential homes. Alzheimer Disease and Associated Disorders, 20(3), 160-165.

Spector, A., Orrell, M., Davies, S., \& Woods, B. (2001). Can reality orientation be rehabilitated? Development and piloting of an evidence-based programme of cognition-based therapies for people with dementia. Neuropsychological Rehabilitation, 11(3-4), 377-397. Retrieved from http://web.a.ebscohost.com. helicon.vuw.ac.nz/ehost/pdfviewer/pdfviewer?sid=5de64f0b-fe70-4221-9ffa$07 \mathrm{e} 1162 \mathrm{e} 80 \mathrm{eb} \% 40$ sessionmgr4006\& vid $=0 \&$ hid $=4212$

Spector, A., Thorgrimsen, L., Woods, B., Royan, L., Davies, S., Butterworth, M., \& Orrell, M. (2003). Efficacy of an evidence-based cognitive stimulation therapy programme for people with dementia: Randomised controlled trial. British Journal of Psychiatry, 183(248-252).

Spijker, A., Vernooij-Dassen, M., Vasse, E., Adang, E., Wollersheim, H., Grol, R., \& Verhey, F. (2008). Effectiveness of nonpharmacological interventions in delaying the institutionalization of patients with dementia: A meta-analysis. Journal of the American Geriatrics Society, 56(6), 1116-1128. doi: 10.1111/j.15325415.2008.01705.x

Stake, R.E. (2003). Case studies. In N.K. Denzin \& Y.S. Lincoln (Eds.), Strategies for qualitative inquiry ( $2^{\text {nd }}$ ed.). London, UK: Sage publications.

Stephan, B \& Brayne, C. Prevalence and projections of dementia. In M Downs, \& B, Bowers (Eds). Excellence in dementia care: Research into practice (2nd ed.). (pp. 3-19). Berkshire, England: Open University Press.

Stineman, M.G., Streim, J.E., Pan, Q., Kurichi, J.E., Schüssler-Fiorenza Rose, S.M., \& Xie, D. (2014). Activity limitation stages empirically derived for activities of daily living (ADL) and instrumental ADL in the U.S. Adult community-dwelling Medicare population. Physical Medicine and Rehabilitation, 6(11), 976-987. doi: 10.1016/j.pmrj.2014.05.001

Strack, F., Martin, L.L., \& Stepper, S. (1988). Inhibiting and facilitating conditions of the human smile: A nonobtrusive test of the facial feedback hypothesis. Journal of Personality and Social Psychology, 54(5), 768.retrieved from https://scholar.google.co.nz/scholar?hl=en\&q=facial+feedback+hypothesis\&btnG $=\&$ as_sdt $=1 \% 2 \mathrm{C} 5 \&$ as_sdtp $=$

Sugarman, J., Roter, D., Cain, C., Wallace, R., Schmechel, D., \& Welsh-Bohmer, K.A. (2007). Proxies and consent discussions for dementia research. American Geriatric Society, 55, 556-561. doi: 10.1111/j.1532-5415.2007.01101.x

Sun, Y., Lai, M.S., Lu, C.J., \& Chen, R.C. (2008). How long can patients with mild or moderate Alzheimer's dementia maintain both the cognition and the therapy of cholinesterase inhibitors: A national population-based study. European Journal of Neurology, 15(3), 278-283. doi: 10.1111/j.1468-1331.2007.02049.x 
Sutton, J. (2010). Memory. https://leibniz.stanford.edu/friends/info/copyright/

Swaffer, K. (2014). Dementia: Stigma, language, and dementia-friendly. Dementia, 13(6), 709-716. doi: 10.1177/1471301214548143

Tailby, R., \& Haslam, C. (2003). An investigation of errorless learning in memoryimpaired patients: Improving the technique and clarifying the theory. Neuropsychologia, 41, 1230-1240.

Tarraga, L., Boada, M., Modinos, G., Espinosa, A., Diego, S., Guitart, M., . . Becker, T. (2006). A randomised pilot study to assess the efficacy of an interactive, multimedia tool for cognitive stimulation in Alzheimer's disease. Journal of Neurology, Neurosurgery \& Psychiatry, 77(10), 1116-1121. doi: 10.1136/jnnp.2005.086074

Taulbee, L. R., \& Folsom, J. C. (1966). Reality orientation for geriatric patients. Psychiatric Services, 17(5), 133-135.

Teel, C.S. (2004). Rural practitioners' experiences in dementia diagnosis and treatment. Aging and Mental Health, 8(5), 422-429.

Terrace, H.S. (1963). Discrimination learning with and without "errors." Journal of the Experimental Analysis of Behavior, 6(1), 1-27.

Terrace, H.S. (1981). This weeks citation classic: Terrace H S. Discrimination learning with and without "errors." Journal of the Experimental Analysis of Behavior, 6, 127. Retrieved from http://garfield.library.upenn. edu/classics1981/ A1981MJ55900001.pdf

The Lancet Neurology. (2014). G8 dementia summit: A chance for united action. The Lancet Neurology. 1(1), 13. Retrieved from http://linkinghub.elsevier.com / retrieve/pii/S1474442213702758

Thivierge, S., Simard, M., Jean, L., \& Grandmaison, É. (2008). Errorless learning and spaced retrieval techniques to relearn instrumental activities of daily living in mild Alzheimer's disease: A case report study. Neuropsychiatric Disease and Treatment, 4(5), 987-999.

Thomas, H. (2010). Mini-Mental State Examination (MMSE). Retrieved from http://www.patent.co.uk/doctor/Mini-Mental-State -Examination-(MMSE).htm (now deleted from the site)

Twigg, J. (2010) Clothing and dementia: A neglected dimension? Journal of Aging Studies, 24(4), 223-230. doi:10.1016/j.jaging.2010.05.002

UHN (2015). Researchers: Pia Kontos: Senior Scientist, Toronto Rehabilitation Institute (TRI). Retrieved from http://www.uhnres.utoronto.ca/researchers/ profile.php?lookup $=56403$

Urdan, T.C. (2010). Statistics in plain English.(3rd ed.). NY. USA: Routledge.

Vaismoradi, M., Turunen, H., \& Bondas, T. (2013). Content analysis and thematic analysis: Implications for conducting a qualitative descriptive study. Nursing \& Health Science. 15, 398-405. doi: 10.1111/nhs. 12048

van den Berg, S., \& Splaine, M. (2012). Policy brief: Risk factors for dementia. Alzheimer's Disease International. Retrieved from www.alz.co.uk.

van Tilborg, I.A., Kessels, R.P., \& Hulstijn, W. (2011). How should we teach everyday skills in dementia? A controlled study comparing implicit and explicit training methods. Clinical Rehabilitation, 25(7), 638-648.

Verheij, S., Muilwijk, D., Pel, J.J.M., van der Cammen, T.J.M., Mattace-Raso, F.U.S., \& van der Steen, J. (2012). Visuomotor impairment in early-stage Alzheimer's disease: Changes in relative riming of eye and hand movements. Journal of Alzheimer's Disease, 30(1), 131-143.

Viera, A.J., \& Garrett, J.M. (2005). Understanding interobserver agreement: The Kappa statistic. Family Medicine, 37(5), 360-363. 
Walker, J.L. (2012). Research column. The use of saturation in qualitative research. Canadian Journal of Cardiovascular Nursing, 22(2), 37-41.

Ward-Griffin, C., Oudshoorn, A., Clark, K., \& Bol, N. (2007). Mother-adult daughter relationships within dementia care: A critical analysis. Journal of Family Nursing, 13(1), 13-32. doi: 10.1177/1074840706297424

Wells, C.S., \& Wollack, J.A. (2003). An instructor's guide to understanding test reliability. University of Wisconsin, USA. Retrieved from: https://testing.wisc.edu/ Reliability.pdf

Wherton, J.P., \& Monk, A.F. (2008). Technological opportunities for supporting people with dementia who are living at home. International Journal of Human-Computer Studies, 66(8), 571-586. Retrieved from www.elsevier.com/locate/ijhcs

White, H., McConnell, E., Clipp, E., Branch, L.G., Sloane, R., Pieper, C., \& Box, T.L. (2002). A randomized controlled trial of the psychosocial impact of providing internet training and access to older adults. Aging Mental Health, 6(3), 213-221. doi: 10.1080/13607860220142422

Whitmer, R.A., Gunderson, E.P., Quesenberry, C.P., Zhou, J.F., \& Yaffe, K. (2007). Body mass index in midlife and risk of Alzheimer disease and vascular dementia. Current Alzheimer Research, 4, 103-109.

Williams, C.L., \& Parker, C. (2012). Development of an observer rating scale for caregiver communication in persons with Alzheimer's disease. Issues in Mental Health Nursing, 33(4), 244-250. doi: 10.3109/01612840.2011.653040

Winzelberg, G.S., Williams, C.S., Preisser, J.S., Zimmerman, S., \& Sloane, P.D. (2005). Factors associated with nursing assistant quality-of-life ratings for residents with dementia in long-term care facilities. The Gerontologist, 45, 106-114.

Woods, B., Aguirre, E., Spector, A.E., \& Orrell, M. (2012). Cognitive stimulation to improve cognitive functioning in people with dementia. Cochrane Database Systematic Review, 2, CD005562. doi:10.1002/14651858. CD005562.pub2

Woods, B., Thorgrimsen, L., Spector, A., Royan, L., \& Orrell, M. (2006). Improved quality of life and cognitive stimulation therapy in dementia. Aging \& Mental Health, 10(3), 219-226. doi: http://dx.doi.org/10.1080/ 13607860500431652

Wray, N., Markovic, M., \& Manderson, L. (2007). "Researcher saturation": The impact of data triangulation and intensive-research practices on the researcher and qualitative research process. Qualitative Health Research, 17(10), 1392-1402. doi: 10.1177/1049732307308308

Yamaguchi, H., Maki, Y., \& Yamagami, T. (2010). Overview of non-pharmacological intervention for dementia and principles of brain-activating rehabilitation. Psychogeriatrics, 10(4), 206-213. doi: 10.1111/j.1479-8301.2010.00323.x.

Yin, R.K. (2014). Case study research: Designs and methods (5 ${ }^{\text {th }}$ ed.). London, England: Sage Publications.

Zanetti, O., Binetti, G., Magni, E., Rozzini, L., Bianchetti, A., \& Trabucchi, M. (1997). Procedural memory stimulation in Alzheimer's disease: Impact of a training programme. Acta Neurologica Scandinavica, 95(3), 152-157. doi: 10.1111/j.16000404.1997.tb00087.x

Zickuhr, K., \& Madden, M. (2012). Older adults and internet use. Pew Internet \& American Life Project, 6. Retrieved from http://www.sainetz.at/dokumente/ Older_adults_and_internet_use_2012.pdf

Zygouris, S., \& Tsolaki, M. (2015). Computerized cognitive testing for older adults: A review. American Journal of Alzheimers Disease and Other Dementias, 30(1), 1328. doi: $10.1177 / 1533317514522852$ 